\title{
Advanced Layered Division Multiplexing Technologies for Next-Gen Broadcast
}

\author{
Departamento de Comunicaciones \\ Universitat Politècnica de València \\ A thesis for the degree of \\ PhD in Telecommunications Engineering \\ Valencia, June 2018
}

Author: Eduardo Garro Crevillén

Supervisors: Dr. David Gómez Barquero Prof. Narcís Cardona Marcet 

To Lucas' mom 



\section{Abstract}

Since the beginning of the 21st century, terrestrial broadcasting systems have been blamed of an inefficient use of the allocated spectrum. To increase the spectral efficiency, digital television Standards Developing Organizations (SDOs) settled to develop the technical evolution of the first-generation Digital Terrestrial Television (DTT) systems. Among others, a primary goal of nextgeneration DTT systems (European DVB-T2 and U.S. ATSC 3.0) is to simultaneously provide TV services to mobile and fixed devices. The major drawback of this simultaneous delivery is the different requirement of each reception condition. To address these constraints different multiplexing techniques have been considered. While DVB-T2 fulfilled the simultaneous delivery of the two services by Time Division Multiplexing (TDM), ATSC 3.0 adopted the Layered Division Multiplexing (LDM) technology. LDM can outperform TDM and Frequency Division Multiplexing (FDM) by taking advantage of the Unequal Error Protection (UEP) ratio, as both services, namely layers, utilize all the frequency and time resources with different power levels. At receiver side, two implementations are distinguished, according to the intended layer. Mobile receivers are only intended to obtain the upper layer, known as Core Layer (CL). In order not to increase their complexity compared to single layer receivers, the lower layer, known as Enhanced Layer (EL) is treated as an additional noise on the CL decoding. Fixed receivers, increase their complexity, as they should performed a Successive Interference Cancellation (SIC) process on the CL for getting the EL. To limit the additional complexity of fixed receivers, the LDM layers in ATSC 3.0 are configured with different error correction capabilities, but share the rest of physical layer parameters, including the Time 
Interleaver (TIL), the Pilot Pattern (PP), the Fast Fourier Transform (FFT) size, and the Guard Interval (GI).

This dissertation investigates advanced technologies to optimize the LDM performance. A demapping optimization for the two LDM layers is first proposed. A capacity increase is achieved by the proposed algorithm, which takes into account the underlying layer shape in the demapping process. Nevertheless, the number of Euclidean distances to be computed can be significantly increased, contributing to not only more complex fixed receivers, but also more complex mobile receivers. Next, the most suitable ATSC 3.0 pilot configuration for LDM is determined. Considering the two layers share the same PP a trade-off between pilot density (CL) and data overhead (EL) arises. From the performance results, it is recommended the use of a not very dense PP, as they have been already designed to cope with long echoes and high speeds. The optimum pilot amplitude depends on the channel estimator at receivers (e.g. the minimum amplitude is recommended for a Wiener implementation, while the maximum for a FFT implementation).

The potential combination of LDM with three advanced technologies that have been adopted in ATSC 3.0 is also investigated: Multi-Radio Frequency Channel (MultiRF) technologies, distributed Multiple-Input Single-Output (MISO) schemes, and co-located Multiple-Input Multiple-Output (MIMO) schemes. The potential use cases, the transmitter and receiver implementations, and the performance gains of the joint configurations are studied for the two LDM layers. The additional constraints of combining LDM with the advanced technologies is considered admissible, as the greatest demands (e.g. a second receiving chain) are already contemplated in ATSC 3.0. Significant gains are found for the mobile layer at pedestrian reception conditions thanks to the frequency diversity provided by MultiRF technologies. The conjunction of LDM with distributed MISO schemes provides significant performance gains on SFNs for the fixed layer with Alamouti scheme. Last, considering the complexity in the mobile receivers and the CL performance, the recommended joint configuration is MISO in the CL and MIMO in the EL. 


\section{Resumen}

Desde comienzos del siglo XXI, los sistemas de radiodifusión terrestre han sido culpados de un uso ineficiente del espectro asignado. Para aumentar la eficiencia espectral, los organismos de estandarización (SDOs) de television (TV) digital comenzaron a desarrollar la evolución técnica de los sistemas de Televisión Digital Terrestre (TDT) de primera generación. Entre otros, uno de los objetivos principales de los sistemas de TDT de próxima generación (el europeo DVB-T2 y el estadounidense ATSC 3.0) es proporcionar simultáneamente servicios de TV a dispositivos móviles y fijos. El principal inconveniente de esta entrega simultánea son los diferentes requisitos de cada condición de recepción. Para abordar estas limitaciones, se han considerado diferentes técnicas de multiplexación. Mientras que DVB-T2 acomete la entrega simultánea de los dos servicios mediante Multiplexación por División en Tiempo (TDM), ATSC 3.0 adoptó la Multiplexación por División en Capas (LDM). LDM puede superar a TDM y la Multiplexación por División en Frecuencia (FDM) al aprovechar la relación de Protección de Error Desigual (UEP), ya que ambos servicios, llamados capas, utilizan todos los recursos de frecuencia y tiempo con diferentes niveles de potencia. En el lado del receptor, se distinguen dos implementaciones, de acuerdo con la capa a decodificar. Los receptores móviles solo están destinados a obtener la capa superior, conocida como Core Layer (CL). Para no aumentar su complejidad en comparación con los receptores de capa única, la capa inferior, conocida como Enhanced Layer (EL), es tratada como un ruido adicional en la decodificación. Los receptores fijos aumentan su complejidad, ya que deben realizar un proceso de Cancelación de Interferencia (SIC) sobre la CL para obtener la EL. Para limitar la complejidad adicional de los receptores fijos, las capas de LDM en ATSC 3.0 están configuradas con diferentes 
capacidades de corrección, pero comparten el resto de bloques de la capa física, incluido el Entrelazador Temporal (TIL), el Patrón de Pilotos (PP), el tamaño de Transformada Rápida de Fourier (FFT) y el Intervalo de Guarda (GI).

Esta disertación investiga tecnologías avanzadas para optimizar el rendimiento de LDM. Primero se propone una optimización del proceso de demapeo para las dos capas de LDM. El algoritmo propuesto logra un aumento de capacidad, al tener en cuenta la forma de la EL en el proceso de demapeo de la CL. Sin embargo, el número de distancias Euclidianas a computar puede aumentar significativamente, conduciendo no solo a receptores fijos más complejos, sino también a receptores móviles más complejos. A continuación, se determina la configuración de piloto ATSC 3.0 más adecuada para LDM. Teniendo en cuenta que las dos capas comparten el mismo PP, surge una contrapartida entre la densidad de pilotos (CL) y la redundancia sobre los datos (EL). A partir de los resultados de rendimiento, se recomienda el uso de un PP no muy denso, ya que ya han sido diseñados para hacer frente a ecos largos y altas velocidades. La amplitud piloto óptima depende del estimador de canal en los receptores (ej., se recomienda la amplitud mínima para una implementación Wiener, mientras que la máxima para una implementación FFT).

También se investiga la potencial transmisión conjunta de LDM con tres tecnologías avanzadas adoptadas en ATSC 3.0: las tecnologías de agregación de Múltiples canales RF (MultiRF), los esquemas de Múltiples-Entradas Única-Salida (MISO) distribuido y los de Múltiples-Entradas MúltiplesSalidas (MIMO) colocalizado. Se estudian los potenciales casos de uso, los aspectos de implementación del transmisor y el receptor, y las ganancias de rendimiento de las configuraciones conjuntas para las dos capas de LDM. Las restricciones adicionales de combinar LDM con las tecnologías avanzadas se consideran admisibles, ya que las mayores demandas ya están contempladas en ATSC 3.0 (ej., una segunda cadena de recepción). Se obtienen ganancias significativas para la capa móvil en condiciones de recepción peatonal gracias a la diversidad en frecuencia proporcionada por las tecnologías MultiRF. La conjunción de LDM con esquemas de MISO distribuidos proporciona ganancias de rendimiento significativas en redes SFN para la capa fija con el esquema de Alamouti. Por último, teniendo en cuenta la complejidad en los receptores móviles y el rendimiento de la CL, la configuración conjunta recomendada es MISO en la CL y MIMO en la EL. 


\section{Resum}

Des de començaments del segle XXI, els sistemes de radiodifusió terrestre han sigut culpats d'un ús ineficient de l'espectre assignat. Per a augmentar l'eficiència espectral, els organismes d'estandardització (SDOs) de televisió (TV) digital van començar a desenvolupar l'evolució tècnica dels sistemes de Televisió Digital Terrestre (TDT) de primera generació. Entre altres, un dels objectius principals dels sistemes de TDT de pròxima generació (l'europeu DVB-T2 i el nord-americà ATSC 3.0) és proporcionar simultàniament serveis de TV a dispositius mòbils i fixos. El principal inconvenient d'aquest lliurament simultani són els diferents requisits de cada condició de recepció. Per a abordar aquestes limitacions, s'han considerat diferents tècniques de multiplexació. Mentre que DVB-T2 escomet el lliurament simultani dels dos serveis mitjançant Multiplexació per Divisió en Temps (TDM), ATSC 3.0 va adoptar la Multiplexació per Divisió en Capes (LDM). LDM pot superar a TDM i la Multiplexació per Divisió en Freqüència (FDM) en aprofitar la relació de Protecció d'Error Desigual (UEP), ja que tots dos serveis, cridats capes, utilitzen tots els recursos de freqüència i temps amb diferents nivells de potència. En el costat del receptor, es distingeixen dues implementacions, d'acord amb la capa a decodificar. Els receptors mòbils solament estan destinats a obtenir la capa superior, coneguda com Core Layer (CL). Per a no augmentar la seua complexitat en comparació amb els receptors de capa única, la capa inferior, coneguda com Enhanced Layer (EL), és tractada com un soroll addicional en la decodificació. Els receptors fixos augmenten la seua complexitat, ja que han de realitzar un procés de Cancel-lació d'Interferència (SIC) sobre la CL per a obtenir l'EL. Per a limitar la complexitat addicional dels receptors fixos, les capes de LDM en ATSC 3.0 estan configurades amb diferents capacitats de 
correcció, però comparteixen la resta de blocs de la capa física, inclòs el Entrellaçador Temporal (TIL), el Patró de Pilots (PP), la grandària de Transformada Ràpida de Fourier (FFT) i l'Interval de Guarda (GI).

Aquesta dissertació investiga tecnologies avançades per a optimitzar el rendiment de LDM. Primer es proposa una optimització del procés de demapeo per a les dues capes de LDM. L'algoritme proposat aconsegueix un augment de capacitat, en tenir en compte la forma de l'EL en el procés de demapeo de la CL. No obstant açò, el nombre de distàncies Euclidianes a computar pot augmentar significativament, conduint NO sols a receptors fixos més complexos, sinó també a receptors mòbils més complexos. A continuació, es determina la configuració de pilot ATSC 3.0 més adequada per a LDM. Tenint en compte que les dues capes comparteixen el mateix PP, es produeix una contrapartida entre la densitat de pilots (CL) i la redundància sobre les dades (EL). A partir dels resultats de rendiment, es recomana l'ús d'un PP no gaire dens, ja que ja han sigut dissenyats per a fer front a ecos llargs i altes velocitats. L'amplitud pilot òptima depèn de l'estimador de canal en els receptors (ex., es recomana l'amplitud mínima per a una implementació Wiener, mentre que la màxima per a una implementació FFT).

També s'investiga la potencial transmissió conjunta de LDM amb tres tecnologies avançades adoptades en ATSC 3.0: les tecnologies d'agregació de Múltiples canals RF (MultiRF), els esquemes de Múltiples-Entrades Única-Eixida (MISO) distribuït i els de Múltiples-Entrades Múltiples-Eixides (MIMO) colocalitzat. S'estudien els potencials casos d'ús, els principals aspectes d'implementació del transmissor i el receptor, i els guanys de rendiment de les configuracions conjuntes per a les dues capes de LDM. Les restriccions addicionals de combinar LDM amb les tecnologies avançades es consideren admissibles, ja que les majors demandes ja estan contemplades en ATSC 3.0 (ex., una segona cadena de recepció). S'obtenen guanys significatius per a la capa mòbil en condicions de recepció per als vianants gràcies a la diversitat en freqüència proporcionada per les tecnologies MultiRF. La conjunció de LDM amb esquemes MISO distribuïts proporciona guanys de rendiment significatius en xarxes SFN per a la capa fixa amb l'esquema d'Alamouti. Finalment, tenint en compte la complexitat en els receptors mòbils i el rendiment de la CL, la configuració conjunta recomanada és MISO en la CL i MIMO en l'EL. 


\section{Acknowledgements}

Firstly, I would like to express my sincere gratitude for the continuous support of my Ph.D study to my advisor Dr. David Gómez Barquero. Thanks for the continuous advices, patience and motivation. I could not imagine a better advisor for my Ph.D study. I would like to thank as well to Prof. Narcís Cardona Marcet for letting me go inside this research group and bring me the chance to go after my Ph.D.

Besides my official advisor, I cannot forget Dr. Sung-Ik Park for being my "uncredited" advisor. His uninterrupted motivation during these 4 years have been a rewarding experience. In addition, I would also thank to the rest of ETRI research institute members, where I had the pleasure to make my Ph.D. stay. I cannot forget to Dr. Jae-Young Lee, Sunhyoung Kwon, Bo-Mi Lim, Dr. Heung-Mook Kim and Dr. Namho Hur.

It is also my pleasure to thank Takuya Shitomi, Dr. Peter Klenner and Prof. Jeongchang Kim for all the valuable comments and discussions on channel estimation, information-theory and implementation aspects. Thank for the support received on the papers development.

Special thanks to the thesis external reviewers: Dr. Jon Montalbán, Dr. Liang Zhang, and Dr. Dazhi He, for their insightful comments. I thank also Prof. Pablo Angueira, Dr. Yiyan Wu and Prof. Alberto González for being members of the committee.

I would like to thank all the Mobile Communications Group (MCG - Boys and Girls) of the Insitute of Telecommunications and Multimedia Applications 
(iTEAM) for providing a nice working atmosphere: Manuel, Jose Luís, Charlie Barjau, Gerardo, Irene, Alejandro, Sergio, Charlie Herranz, David García, David Martín-Sacristán, Tere, Conchi, Alicia, Sofía, Martina, Josetxo, Dani and Sandra. Special mention to former members of the group Jefferson, Jaime, David Vargas, Jordi and Carlos Andrew.

To my life-coaches, my parents Eduardo and Toñi, because I owe you everything. To my beloved brother Miki and my sister Mar. And last, but it does not mean least, to my wife Patri for being there whenever I needed you. One last special mention to the new baby in home. 


\section{Contents}

Abstract $\quad$ iii

Contents $\quad$ xiii

Acronyms $\quad$ xix

1 Introduction 1

1.1 Terrestrial broadcasting development. . . . . . . . . . . . 1

1.2 Simultaneous Mobile and Fixed Services Delivery . . . . . . . . . 6

1.2.1 Problem Statement . . . . . . . . . . . . . . . . . . . . . . . . 8

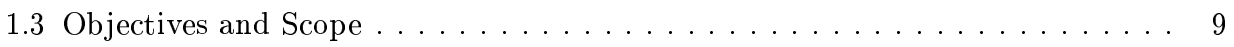

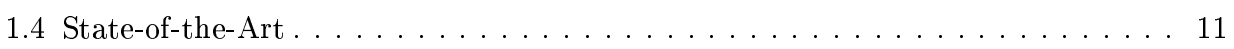

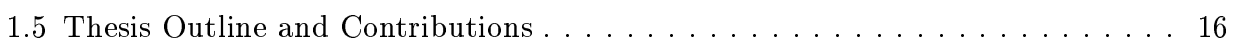

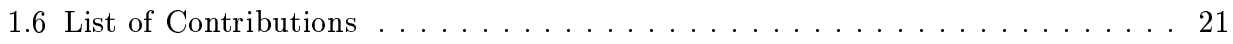

2 Optimal Demappers for Multi-Layer Broadcast Systems 25

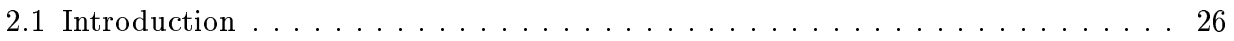


2.2 Constellation Demapper Alternatives in Multi-layer Systems . . . . . . . . . 28

2.2 .1 GD Apprach . . . . . . . . . . . . . . . . . . . . . . . . . 28

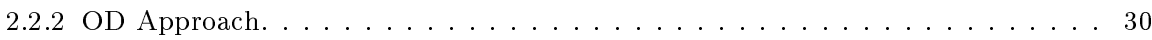

2.2 .3 Demappers Complexity. . . . . . . . . . . . . . . . . . . . . . 31

2.3 Information-Theoretical Analysis of BICM systems . . . . . . . . . . . 32

2.3.1 Error Exponent Analysis . . . . . . . . . . . . . . . . . . . . . . . . . 32

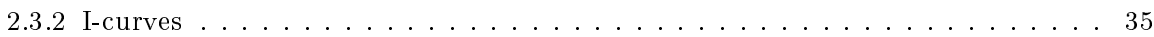

2.3.3 Generalized Mutual Information Analysis . . . . . . . . . . . . 37

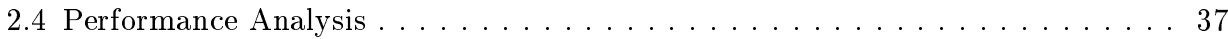

2.4.1 Performance of top-layer signal $\left(x_{t}\right)$ in AWGN channel . . . . . . . . . 38

2.4.2 Performance of top-layer signal $\left(x_{t}\right)$ in i.i.d. Rayleigh channel . . . . . . . . . 41

2.4.3 Performance of bottom-layer signal $x_{b} \ldots \ldots \ldots \ldots \ldots \ldots$

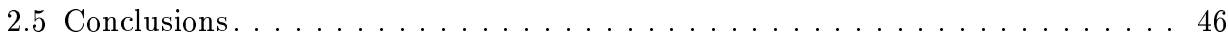

3 Scattered Pilot Performance and Optimization for ATSC $3.0 \quad 49$

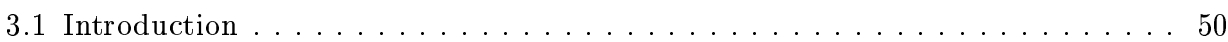

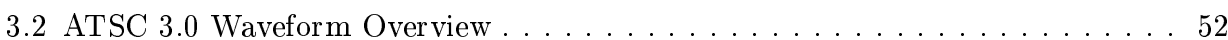

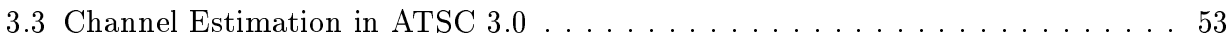

3.3.1 Scattered Pilot Configuration assumed at Transmitters . . . . . . . . . . . 53

3.3.2 Channel Estimator implemented at Receivers . . . . . . . . . . . . . . . 58

3.3.3 Channel Estimation in LDM . . . . . . . . . . . . . . . . . . . . . . 60

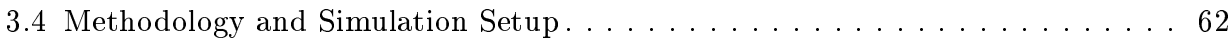

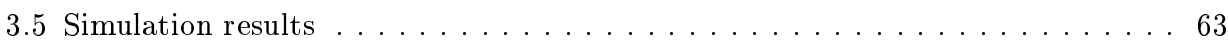

3.5.1 FFT size and TIL depth impact in TDM systems . . . . . . . . . . . 63

3.5.2 SP and Pilot Boosting impact for TDM systems . . . . . . . . . . . . . 64

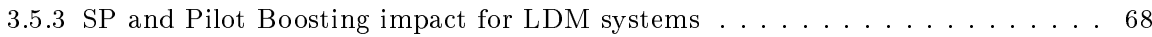

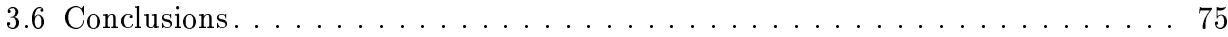

\section{Layered Division Multiplexing with Multi-Radio Frequency Chan-} nel Technologies $\quad 77$

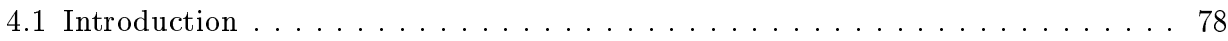

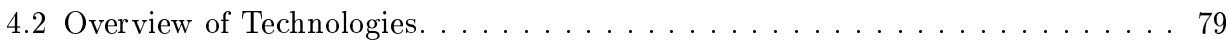

4.2 .1 Layered Division Multiplexing $(\mathrm{LDM}) \ldots \ldots \ldots \ldots \ldots \ldots$ 
4.2 .2 Channel Bonding $(\mathrm{CB}) \ldots \ldots \ldots \ldots \ldots$. . . . . . . . . . . . . 81

4.2 .3 Time Frequency Slicing $(\mathrm{TFS}) \ldots \ldots \ldots \ldots$. . . . . . . . . . . . 82

4.3 Use Cases for LDM and MultiRF Channel . . . . . . . . . . . . . . . . 83

4.3 .1 Increased Peak Service Data-rate. . . . . . . . . . . . . . . . . . . . . 84

4.3 .2 StatMux Gain . . . . . . . . . . . . . . . . . . . 84

4.3 .3 Increased RF Performance. . . . . . . . . . . . . . . . . . . . . 85

4.4 Implementation Aspects of LDM with MultiRF Channel Technologies . . . . . 86

$4.4 .1 \mathrm{LDM}$ with TFS . . . . . . . . . . . . . . . . . . 86

4.4 .2 LDM with Channel Bonding . . . . . . . . . . . . . . . . . 89

4.5 Methodology and Simulation Setup. . . . . . . . . . . . . . 90

4.6 LDM and MultiRF Channel Performance Evaluation . . . . . . . . . . . . . . 93

4.6.1 LDM EL and MultiRF performance for fixed reception. Influence of the TIL

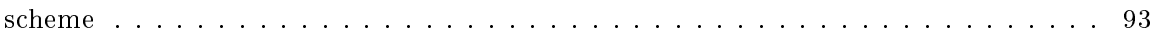

4.6.2 LDM CL and MultiRF performance for mobile reception . . . . . . . . . . . . 94

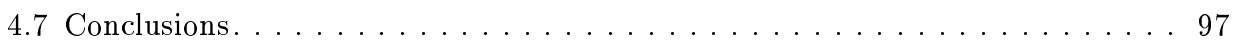

\section{Layered Division Multiplexing with Distributed Multiple-Input} Single-Output Schemes 99

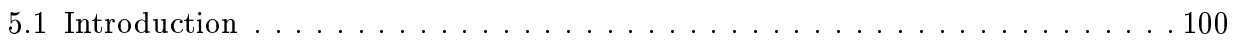

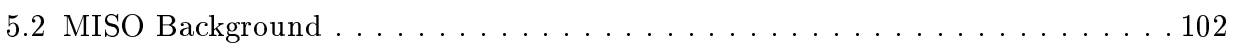

5.2 .1 Frequency pre-distortion . . . . . . . . . . . . . . . . . . . . 102

5.2 .2 Space-Time/Frequency Block Coding $(\mathrm{ST} / \mathrm{FBC}) \ldots \ldots$. . . . . . . . . . 103

5.3 Implementation Aspects of LDM with MISO schemes on ATSC 3.0 . . . . . 105

5.3 .1 LDM with MISO TDCFS . . . . . . . . . . . . . 105

5.3 .2 LDM with MISO Alamouti . . . . . . . . . . . . . . . . 106

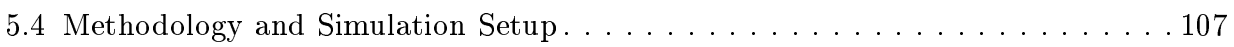

5.4 .1 Methodology . . . . . . . . . . . . . . . . . . . 107

5.4 .2 Transmission Setup . . . . . . . . . . . . . . . . . 108

$5.4 .3 \mathrm{SFN}$ scenarios . . . . . . . . . . . . . . . . . . . 109

5.4 .4 Receiver configuration . . . . . . . . . . . . . . . . . . 110

$5.5 \mathrm{LDM}$ and distributed MISO Performance Evaluation . . . . . . . . . . . 113

5.5 .1 Core Layer Performance . . . . . . . . . . . . . . . . . . . . . . . . 115

5.5 .2 Enhanced Layer Performance . . . . . . . . . . . . . . . . . . . 117

5.5 .3 Influence of Injection Level $(\Delta) \ldots \ldots \ldots$. . . . . . . . . . . . . . . . 120 
5.6 Conclusions. . . . . . . . . . . . . . . . . . . . . . . . . . . . . . . 121

\section{Layered Division Multiplexing with Co-Located Multiple-Input} Multiple-Output Schemes

6.1 Introduction . . . . . . . . . . . . . . . . . . . . . 124

6.2 MIMO in Terrestrial Broadcasting . . . . . . . . . . . . . . . 125

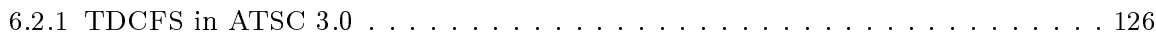

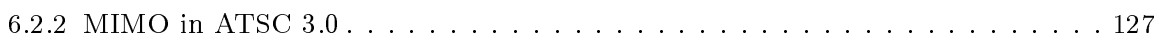

6.3 Potential co-located MIMO and LDM use cases . . . . . . . . . . . . . . . 129

6.3 .1 Mobile receivers with one antenna . . . . . . . . . . . . . . . . . . . . 130

6.3 .2 Mobile receivers with two antennas . . . . . . . . . . . . . . . . . . 130

6.4 Implementation Aspects of LDM with co-located MIMO schemes. . . . . . . . . 132

6.4 .1 Mobile receivers block diagrams . . . . . . . . . . . . . . . . . . . . . . . 132

6.4 .2 Fixed receivers block diagrams . . . . . . . . . . . . . . . . . . . 133

6.4.3 Receivers blocks complexity . . . . . . . . . . . . . . . . . . . . . 134

6.5 Methodology and Simulation Setup. . . . . . . . . . . . . . . . 140

6.5 .1 Transmitter Setup . . . . . . . . . . . . . . . . . . . . . . . . 140

6.5.2 Channel models . . . . . . . . . . . . . . . . . . . . . . . . . . . . . . . . 140

6.5 .3 Receiver Setup . . . . . . . . . . . . . . . . . . . . . . . . 141

6.6 LDM and co-located MIMO Performance Evaluation . . . . . . . . . . . . . . . 142

6.6.1 Mean Square Error of Channel Estimation . . . . . . . . . . . . . . . . . . . 142

6.6 .2 CL Performance . . . . . . . . . . . . . . . . . . . . . . . . . . . . . . . . . 144

6.6 .3 EL Performance . . . . . . . . . . . . . . . . . . . . . . . . . . . . . . . . . 148

6.7 Conclusions. . . . . . . . . . . . . . . . . . . . . . . . . . . . . . 149

7 Results Discussions 153

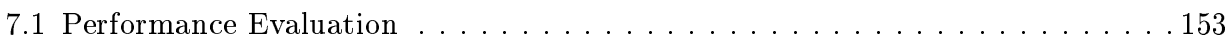

7.1 .1 BICM Impact . . . . . . . . . . . . . . . . . . . . . . . . . . . . . . . 154

7.1 .2 TIL Impact . . . . . . . . . . . . . . . . . . . . . . . 156

7.1 .3 PP Impact . . . . . . . . . . . . . . . . . . . . . . . . . . . 159

7.1 .4 MISO Impact. . . . . . . . . . . . . . . . . . . . . . . . . . . . 162

7.1 .5 FFT Impact . . . . . . . . . . . . . . . . . . . . . . . . . . . . . 164

7.1 .6 MIMO Impact . . . . . . . . . . . . . . . . . . . . 165 
7.2 Implementation Aspects . . . . . . . . . . . . . . . . . 168

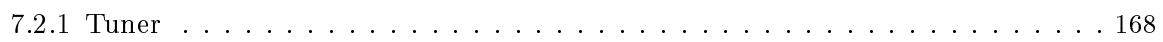

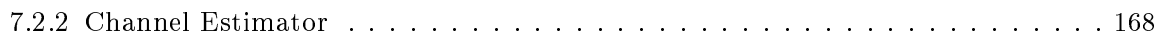

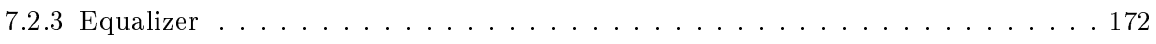

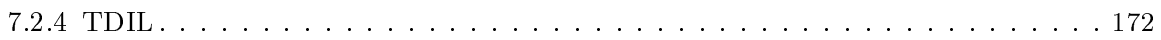

7.2 .5 Cell Re-Exchanger. . . . . . . . . . . . . . . . . . . . . . . . 173

7.2 .6 LDM Buffer. . . . . . . . . . . . . . . . . . . . . . . . . . 173

7.2 .7 Demapping . . . . . . . . . . . . . . . . . . . . . . . 174

7.2 .8 LDPC decoding . . . . . . . . . . . . . . . . . . . . . 174

7.2 .9 MIMO . . . . . . . . . . . . . . . . . . . . . . . . . . . . . . 174

8 Conclusions and Future Work 177

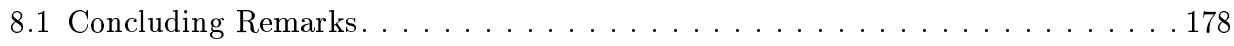

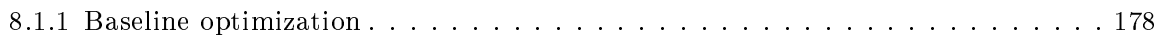

8.1.2 Joint LDM and advanced technologies transmissions . . . . . . . . . . . . 180

8.2 Recommendations for Advanced LDM transmissions . . . . . . . . . . . . . . 182

8.3 Future Research Topics . . . . . . . . . . . . . . . . . . . . . . . . 184

8.3.1 Further studies of LDM with co-located MIMO schemes . . . . . . . . . . . . 184

8.3.2 N-LDM and LDM Segmentation . . . . . . . . . . . . . . . . . . . . 184

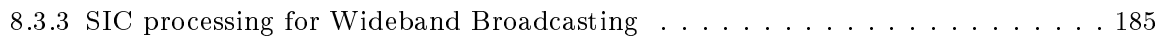

8.3.4 NOM for 5G Mobile Systems . . . . . . . . . . . . . . . . . 186

$\begin{array}{ll}\text { Bibliography } & 187\end{array}$ 



\section{Acronyms}

3GPP Third Generation Partnership Project

5G 5th Generation

8-VSB 8 Level Vestigial Side Band

AGC Automatic Gain Control

ARIB Association of Radio Industries and Businesses

ATSC Advanced Television Systems Committee

ATSC 3.0 ATSC - Third Generation

AVC Advanced Video Coding

AWGN Additive White Gaussian Noise

BCH Bose Chadhuri Hocquenghem

BDIL Bit De-Interleaver

BER Bit Error Rate

BICM Bit-Interleaved Coded Modulation

BIL Bit Interleaver

BW Bandwidth 
CB Channel Bonding

CFR Channel Frequency Response

CL Core Layer

CLI Cross-Layer Interference

CR Coding Rates

CIL Convolutional Interleaver

CloudTxn Cloud Transmission

COFDM Coded Orthogonal Frequency-Division Multiplexing

DD Digital Dividend

DTMB Digital Terrestrial Multimedia Broadcast

DTT Digital Terrestrial Television

DTV Digital TV

DVB Digital Video Broadcasting

DVB-F1 DVB Fixed Rice

DVB-NGH Digital Video Broadcasting - Next Generation Handheld

DVB-T Digital Video Broadcasting - Terrestrial

DVB-T2 DVB - Terrestrial Second Generation

EGC Equal Gain Combining

EL Enhanced Layer

eMBMS evolved Multimedia Broadcast/Multicast Service

eSFN enhanced SFN

ETSI European Telecommunications Standards Institute

FCC Federal Communications Commission

FDIL Frequency De-Interleaver 
FDM Frequency Division Multiplexing

FEC Forward Error Correction

FEF Future Extension Frames

FFT Fast Fourier Transform

FIL Frequency Interleaver

GD Gaussian Demapping

GI Guard Interval

GMI Generalized Mutual Information

GUR Guard Utilization Ratio

HC Hard Cancellation

HDTV High Definition TV

HEVC High Efficiency Video Coding

ICI Inter-Carrier Interference

i.i.d. independent and identically distributed

$\Delta$ Injection Level

IMT International Mobile Telecommunications

IQ In-Phase and Quadrature

ISDB-T Integrated Services Digital Broadcasting Terrestrial

"One Seg" ISDB-T One-Seg Broadcasting

ISDB-T $_{S B}$ ISDB-T Sound Broadcasting

ISDB-Tmm ISDB-T Multimedia Broadcasting

ISI Inter-Symbol Interference

ITU International Telecommunications Union

LDM Layered Division Multiplexing 
LDPC Low Density Parity Check

LLR Log-Likelihood Ratios

LMMSE Linear Minimum Mean Square Error

LoS Line of Sight

LS Least Square

LTE Long Term Evolution

MFN Multiple Frequency Network

MGM Modified Guilford Model

MIMO Multiple-Input Multiple-Output

MISO Multiple-Input Single-Output

ML Maximum Likelihood

MMSE Minimum Mean Square Error

MNOs Mobile Network Operators

MODCOD Modulation and Coding Rate

MP MIMO Pilot

M-PLP Multiple PLP

MRC Maximum Ratio Combining

MSE Mean Square Error

MultiRF Multi-Radio Frequency Channel

MUST Multi-User Superposition Transmission

NOM Non-Orthogonal Multiplexing

NP Null Pilot

NUC Non-Uniform Constellation

OD Optimum Demapping 
OFDM Orthogonal Frequency-Division Multiplexing

OM Orthogonal Multiplexing

pdf probability density function

PDP Power Delay Profile

PLL Phase Locked Loop

PLP Physical Layer Pipes

PP Pilot Pattern

PTM Point-to-Multipoint

QAM Quadrature Amplitude Modulation

QEF Quasi-Error Free

QPSK Quadrature Phase-Shift Keying

RF Radio Frequency

SC Soft Cancellation

SCM Superposition Coding

SDOs Standard Developing Organizations

SDR Software Defined Radio

SFBC Space-Frequency Block Code

SFN Single Frequency Networks

SHVC Scalable HEVC

SI Study Item

SIC Successive Interference Cancellation

SIMO Single-Input Multiple-Output

SINR Signal-to-Interference-and-Noise Ratio

SISO Single-Input Single-Output 
SM Spatial Multiplexing

SNR Signal-to-Noise Ratio

SP Scattered Pilot

S-PLP Single - PLP

StatMux Statistical Multiplexing

ST/FBC Space Time/Frequency Block Code

T2-Lite DVB-T2 Lite Profile

TDIL Time De-Interleaver

TDCFS Transmit Diversity Code Filter Sets

TDM Time Division Multiplexing

TFS Time-Frequency Slicing

TIL Time Interleaver

TU-6 Typical Urban

TV Television

UEP Unequal Error Protection

UHDTV Ultra High-Definition TV

UPV Universitat Politècnica de València

U.S. United States

UHF Ultra-High Frequency

VHF Very-High Frequency

WH Walsh-Hadamard

WiB Wideband reuse-1

WRC World Radiocommunication Conference

XPD Cross-Polarization Discrimination 
ZF Zero Forcing 

Chapter 1

\section{Introduction}

\subsection{Terrestrial broadcasting development}

\section{The transition from Analogue $T V$ to Digital TV}

Television (TV) has been the most popular communication systems after World War II. From 1950, it has played an important role in the home entertainment, as families and friends gathered around watching historic events. The images were originally transmitted in black and white, but in the mid-1960s, color broadcasting, still using analogue TV was introduced. These analogue transmissions used significant parts of the Ultra-High Frequency (UHF) and Very-High Frequency (VHF) spectrum bands, and, thus, a few limited analogue programs were able to be transmitted. The switch from analogue TV to Digital TV (DTV) conducted to a spectral efficiency increase, and, in turn a better video quality. In addition, a smaller amount of frequency spectrum was needed, so that a higher number of digital programs could be transmitted in the spectrum bandwidth of a single analogue program.

Different first generation Digital Terrestrial Television (DTT) standards, which are still in use, were developed in the 1990s: Advanced Television Systems Committee (ATSC) in United States (U.S.), Mexico, Canada and Korea [1], 
Table 1.1: Main characteristics of first DTT generation systems

\begin{tabular}{|c|c|c|c|c|}
\hline & $A T S C$ & $D V B-T$ & $I S D B-T$ & $D T M B$ \\
\hline Video Coding & MPEG-2 & MPEG2 & MPEG-2 & MPEG-2 \\
\hline \multirow{2}{*}{ Channel Coding } & $\mathrm{RS}$ & $\mathrm{RS}$ & $\mathrm{RS}$ & $\mathrm{RS}$ \\
\hline & Trellis & Convolutional & Convolutional & LDPC \\
\hline Code Rate & $2 / 3$ & $\begin{array}{c}1 / 2,2 / 3,3 / 4 \\
5 / 6,7 / 8\end{array}$ & $\begin{array}{c}1 / 2,2 / 3,3 / 4 \\
5 / 6,7 / 8\end{array}$ & $1 / 2,3 / 4$ \\
\hline \multirow{4}{*}{ Modulation } & \multirow{4}{*}{$\begin{array}{c}8-\mathrm{VSB} \\
16-\mathrm{VSB}\end{array}$} & \multirow{4}{*}{$\begin{array}{c}\text { QPSK } \\
\text { 16QAM } \\
64 \mathrm{QAM}\end{array}$} & DQPSK & QPSK \\
\hline & & & QPSK & 16QAM \\
\hline & & & 16QAM & 32QAM \\
\hline & & & 64QAM & 64QAM \\
\hline$G I$ & - & $\begin{array}{c}1 / 32,1 / 16 \\
1 / 8,1 / 4\end{array}$ & $\begin{array}{c}1 / 32,1 / 16 \\
1 / 8,1 / 4\end{array}$ & $1 / 9,1 / 7,1 / 4$ \\
\hline FFT & - & $2 \mathrm{k}, 8 \mathrm{k}$ & $2 \mathrm{k}, 8 \mathrm{k}, 32 \mathrm{k}$ & $4 \mathrm{k}$ \\
\hline Bit Rate (Mbps) & 19.63 & $5-30$ & $3.65-23.23$ & $4.8-32.5$ \\
\hline
\end{tabular}

Integrated Services Digital Broadcasting Terrestrial (ISDB-T) in Japan and South America (except Colombia) [2], Digital Terrestrial Multimedia Broadcast (DTMB) in China [3], or Digital Video Broadcasting - Terrestrial (DVB-T) in Europe [4]. ATSC adopted the digital modulation 8 Level Vestigial Side Band (8-VSB), but it suffered from multipath distortions. The rest of firstgeneration DTT standards were based on the Coded Orthogonal FrequencyDivision Multiplexing (COFDM) modulation. The concept of Orthogonal Frequency-Division Multiplexing (OFDM) can be assumed as a Frequency Division Multiplexing (FDM) with the additional constraint that the subcarriers are orthogonal to each others. This orthogonality is obtained by making the subcarrier spacing inversely proportional to the useful symbol duration. In COFDM, Forward Error Correction (FEC) is applied to the data. COFDM is capable of handling very strong multipath echoes [5].

Table 1.1 presents the physical layer characteristics of the first DTT generation standards [6]. From the table it can be observed that whereas ATSC has only one operational point, providing a maximum bitrate around 19 Mbps over one $6 \mathrm{MHz}$ channel [7], DVB-T provides a wide range of operational points, 
leading to either very robust signals or to services with a bitrate up to $30 \mathrm{Mbps}$. Nevertheless, they are still far from the theoretical capacity limit [8].

\section{Digital Dividends}

The adoption of the DTT led to released frequency spectrum over the VHF, and UHF bands, formerly assigned to broadcasting services. This spectrum release is known as Digital Dividend (DD) [9], [10]. In the World Radiocommunication Conference (WRC) of 2007, the International Telecommunications Union (ITU) decided to allocate the upper part of the TV broadcasting band to International Mobile Telecommunications (IMT) technologies, like Long Term Evolution (LTE) mobile systems. In particular, ITU Region 2 (i.e., America) released the $700 \mathrm{MHz}$ band (from $694-790 \mathrm{MHz}$ ). On the other hand, the 800 $\mathrm{MHz}$ band (from 790 to $862 \mathrm{MHz}$ ) was released in ITU Regions 1 and 3 (i.e., Europe, Africa, Asia and Oceania) [11]. Moreover, in the WRC of 2015, the ITU also approved the release of the $700 \mathrm{MHz}$ band of ITU Region 1 and 3 to IMT services [12] before 2020. Last, the Federal Communications Commission (FCC) proposed to U.S. broadcasters to voluntarily relinquish their 600 MHz spectrum licenses to Mobile Network Operators (MNOs) [13].

\section{Higher Video Quality Demands}

DTT systems not only have to face the upcoming spectrum scarcity, but also the higher bit rates required for new high-quality content demands, such as High Definition TV (HDTV) or even Ultra High-Definition TV (UHDTV). Progress in video compression schemes allows for significant bit rate reductions in order to provide these additional requirements. For example MPEG4/Advanced Video Coding (AVC), can halve the video bit rate compared to the previous MPEG-2 codec version. Moreover, High Efficiency Video Coding (HEVC) [14] can reduce by $60 \%$ the video bit rate of AVC [15]. Nevertheless, these changes in video coding force the end users to update their TV sets. Taking into account the upcoming spectrum scarcity, the possibility of approaching Shannon's capacity limits, and considering the updated TV sets requirement by final users, the digital television Standard Developing Organizations (SDOs) decided to work on the next-generation DTT standards. 


\section{Next-Generation Digital TV}

Whereas Digital Video Broadcasting (DVB) provided the second generation of their terrestrial standard, DVB - Terrestrial Second Generation (DVB-T2) [16], ATSC has recently published their new generation ATSC - Third Generation (ATSC 3.0) for the U.S. and Korea [17], and Association of Radio Industries and Businesses (ARIB) is internally studying their upcoming ISDB-T2 terrestrial standard for Japan [18], [19].

\section{DVB-T2}

In April 2007, the motivations for the development of a DVB-T evolution were grouped in [20]. Compared with its preceding DVB-T standar, DVB-T2 was designed to at least fulfill a $30 \%$ of spectral efficiency increase for the same Signal-to-Noise Ratio (SNR). The first version was formally announced by European Telecommunications Standards Institute (ETSI) in September 2009 [16].

It is also based on COFDM modulation, but for the spectral efficiency increase, new advanced signal processing techniques were adopted. Regarding channel coding, the FEC was modified from convolutional coding in DVB-T to Low Density Parity Check (LDPC) codes in DVB-T2, which are close to the theoretical limits in Additive White Gaussian Noise (AWGN) channel [21]. Due to the LDPC robustness, DVB-T2 introduces a higher Quadrature Amplitude Modulation (QAM) constellation, 256-QAM. DVB-T2 also provides enough flexibility in its configuration in order to be planned for different reception scenarios (fixed-rooftop, portable and/or mobile), as this was the first requirement in [20]. While DVB-T permitted 2 Fast Fourier Transform (FFT) sizes, 4 Guard Interval (GI) fractions, 1 Pilot Pattern (PP), and 3 channel bandwidths, DVB-T2 increases to 6 FFT sizes, 7 GI fractions, 8 PPs, and 6 channel bandwidths. Moreover, a new block, called Time Interleaver (TIL), which provides time diversity in order to increase robustness against low Doppler channels is also introduced in DVB-T2 [22], [23]. Another primary goal is the simultaneous delivery of mobile and fixed services. To address these constraints, the Time Division Multiplexing (TDM) Physical Layer Pipes (PLP) concept is adopted. Last, an improved Single Frequency Networks (SFN) operation by making use of a Multiple-Input Single-Output (MISO) diversity mechanism is also defined. It is based on a Space-Frequency Block Code (SFBC) antenna group as Alamouti described in [24]. Taking all these features into account, the DVB-T2 SNR operational region under AWGN channel ranges from $\{1 \mathrm{~dB}$ - $22 \mathrm{~dB}\}$, and the minimum and maximum bitrate supported by an $8 \mathrm{MHz}$ 
channel span from to $\{3.9 \mathrm{Mbps}-50.3 \mathrm{Mbps}$, which corresponds to a $60 \%$ spectral efficiency increase over DVB-T [25]).

\section{ATSC 3.0}

ATSC 3.0 is the next-generation U.S. terrestrial broadcasting standard, which outperforms DVB-T2 in terms of coverage and capacity [26]. The standardization process started in October 2013, and its deployment was authorized by FCC in November 2017 [27].

Since ATSC 3.0 is based on COFDM, it has no backwards-compatibility constraint with previous ATSC 1.0 standard. Compared to ATSC 1.0, ATSC 3.0 provides a minimum of $30 \%$ capacity increase at the same operational SNR. Having been developed later, ATSC 3.0 takes advantage of new features and previous experiences of DVB-T2 and the mobile technical specification. Digital Video Broadcasting - Next Generation Handheld (DVB-NGH). As DVB-T2, it makes use of LDPC channel coding, but with a wider range of Coding Rates (CR). It also incorporates advanced features, such as Non-Uniform Constellation (NUC) [28], [29], improved TIL modes [30], Transmit Diversity Code Filter Sets (TDCFS) MISO scheme for improving SFN coverage [31] without additional complexity at receivers. For the simultaneous delivery, not only a TDM by PLPs is incorporated, but also the hot-topic Non-Orthogonal Multiplexing (NOM) mode, defined as Layered Division Multiplexing (LDM) is also adopted. It has also increased the OFDM symbol flexibility by providing up to 16 different PP, with 5 different amplitudes for each one, known as pilot boostings. Three FFT sizes and 12 GI lengths (from 27 to $700 \mu \mathrm{s}$ ) are also adopted. In summary, compared to DVB-T2, the Single-Input Single-Output (SISO) baseline ATSC 3.0 specification provides a wider SNR operational range under AWGN channel (from -6.2 dB to $32 \mathrm{~dB}$ ), and a wider bitrate range (from $1 \mathrm{Mbps}$ to $57 \mathrm{Mbps}$ ). In addition, ATSC 3.0 also allows to double the peak bitrate by bonding 2 Radio Frequency (RF) channels with Channel Bonding (CB) mode [32], [33], or by 2x2 Multiple-Input Multiple-Output (MIMO) transmissions [34], [35]. Table 1.2 presents the physical layer characteristics of the next-generation DTT systems [26]. 
Table 1.2: Main characteristics of next-generation DTT systems

\begin{tabular}{ccc}
\hline \hline & DVB-T2 & ATSC 3.0 \\
\hline \multirow{2}{*}{ Video Coding } & H.264 & H.264 \\
& HEVC & HEVC \\
\hline \multirow{2}{*}{ Channel Coding } & BCH & BCH, CRC \\
& LDPC & LDPC \\
\hline \multirow{2}{*}{ Code Rate } & $1 / 2,3 / 5,2 / 3$, & $\{2-13\} / 15$ \\
& $3 / 4,4 / 5,5 / 6$ & QPSK, $2 \mathrm{D}-16 \mathrm{NUC}$, \\
Modulation & QPSK, $16 \mathrm{QAM}$ & $2 \mathrm{D}-64 \mathrm{NUC}, 2 \mathrm{D}-256 \mathrm{NUC}$, \\
& $64 \mathrm{QAM}, 256 \mathrm{QAM}$ & $1 \mathrm{D}-1024 \mathrm{NUC}, 1 \mathrm{D}-4096 \mathrm{NUC}$ \\
\hline TIL & $\mathrm{BIL}$ & $\mathrm{CIL} \& \mathrm{Hybrid} \mathrm{BIL}+\mathrm{CIL}$ \\
\hline Multiplexing & $\mathrm{TDM}$ & $\mathrm{LDM}, \mathrm{FDM}, \mathrm{TDM}$ \\
\hline MISO & Alamouti & TDCFS \\
\hline \multirow{2}{*}{ GI } & $1 / 128,1 / 32,1 / 16$, & $1 / 32,3 / 64,1 / 16,19 / 256$, \\
& $19 / 256,1 / 8,19 / 128,1 / 4$ & $3 / 32,57 / 512,3 / 16,1 / 8$, \\
& & $19 / 128,1 / 4$ \\
\hline FFT & $1 \mathrm{k}, 2 \mathrm{k}, 4 \mathrm{k}, 8 \mathrm{k}, 16 \mathrm{k}, 32 \mathrm{k}$ & $8 \mathrm{k}, 16 \mathrm{k}, 32 \mathrm{k}$ \\
\hline \hline Bit Rate $($ Mbps) & $5.6-38$ & $1-57$ \\
\hline \hline
\end{tabular}

\subsection{Simultaneous Mobile and Fixed Services Delivery}

As it has been mentioned, among others, one of the primary goals of nextgeneration DTT systems is to simultaneously provide fixed and mobile TV services to fixed-rooftop antennas, and portable or vehicular devices, respectively [26], [36]. The major drawback of this simultaneous delivery is the different requirements of each reception condition. On the one hand, mobile services requires very robust transmissions, in both low SNR and high Doppler distortion environments [37]. On the other hand, fixed receivers are aimed at high data rate services, as the reception conditions are less challenging. Hence, robustness and flexibility are the two main characteristics that should 
be guaranteed for this requirement. To address these constraints, different multiplexing techniques have been considered for next-generation DTT systems.

At first, DVB-T2 introduced the TDM concept of PLP. By means of Multiple PLP (M-PLP), it is possible to configure different transmission characteristics. Thus, it is possible to provide coverage for different reception conditions within the same RF channel [38]. However, the FFT size and the PP, which are directly related with the Doppler tolerance, must be common for all the PLPs. Thus, the simultaneous transmission of stationary and mobile services in DVB-T2 with M-PLP is not optimum. It was solved by the the DVB-T2 Lite Profile (T2-Lite), and the Future Extension Frames (FEF) [39]. They permit an independent FFT size and PP configuration for each multiplexed services in the RF channel. In order to improve mobility performance, DVB developed the handheld evolution of DVB-T2, DVB-NGH [40]. While the core of the system is based on DVB-T2, it introduces new technical elements such as scalable video coding with M-PLP [41], Time-Frequency Slicing (TFS) [33], new code rates and NUC, increased TIL performance or a MIMO profile [42].

In Japan, the ISDB-T multiplexes the services in the frequency domain (FDM). The OFDM signal consists of 13 segments of $428.57 \mathrm{kHz}$, which can be independently encoded and modulated. Whereas the fixed service is transmitted upon 12 segments, the center segment is used for the mobile service transmission. This mode is known as the ISDB-T One-Seg Broadcasting ("One Seg"). However, because of the short bandwidth of "One Seg", the maximum data rate for mobile service is limited to $624.1 \mathrm{kbps}$ [43]. In order to increase the capacity, the evolutions ISDB-T Sound Broadcasting (ISDB-T ${ }_{S B}$ ), and ISDB$T$ Multimedia Broadcasting (ISDB-Tmm) increases the number of segments in the VHF band. However, they are not designed for large coverage areas.

To enhance the Orthogonal Multiplexing (OM) limitations, i.e. TDM or FDM, NOM has emerged as an efficient multiplexing technique for the simultaneous delivery services in wireless transmissions [44]. In power-based NOM, the multiplexed services utilizes all the frequency and time resources but with different power levels, as it is shown in Figure 1.1.

The most representative power-based NOM solution for DTT is the ATSC 3.0's LDM technique [45]. In LDM, the two multiplexed services are independently configured and they are superimposed at different power levels according to a configurable parameter. The mobile service, which suffers of poor channel conditions uses the most robust configuration, while the second multiplexed service, with less challenging fixed-rooftop conditions is used to transmit a high capacity service. LDM can outperform TDM and FDM by taking advan- 


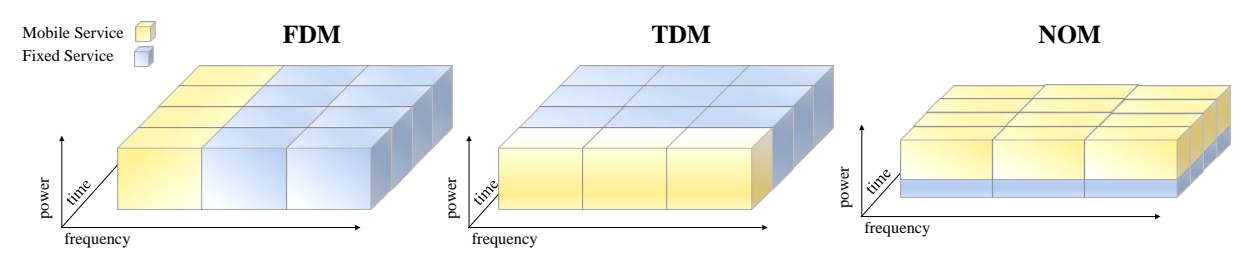

Figure 1.1: Multiplexing modes: FDM, TDM, and NOM.

tage of the Unequal Error Protection (UEP) ratio, as both services, namely layers, utilize all the frequency and time resources with different power levels. At receiver side, despite the initial impressions, LDM suit really well for the simultaneous transmission of mobile and fixed services. On the one hand, mobile terminals are not modified compared to single layer receivers. In order not to increase their complexity, the lower layer is treated as an additional interference. On the other hand, fixed receivers increase their complexity, as they should perform a Successive Interference Cancellation (SIC) process on the upper layer for getting the lower layer [46]. Nevertheless, they do not have the low complexity and low battery consumption requirements of mobile terminals. Moreover, the SIC is carried out really quick, since the upper layer SNR threshold is much lower than the lower layer operational SNR region.

\subsubsection{Problem Statement}

Although the based concept of LDM was proposed long time ago [47], [48], it is still a new technology that has become as one of the hot topics in DTT research field. Different terrestrial broadcasting investigations have been carried out to demonstrate the spectrum efficiency increase versus TDM or FDM [36], [49]-[51]. Moreover, is not only a new technology for terrestrial broadcasting, but also for any wireless communication system. A Downlink NOM solution, called Multi-User Superposition Transmission (MUST), was analyzed for LTEAdvanced Pro [52], as well as an Uplink NOM Study Item (SI), for 5th Generation (5G)-New Radio was approved in March 2017, but delayed to focus on the $5 \mathrm{G}$ main standard (Rel' 15 ). Therefore, further studies and investigations are still pending. This $\mathrm{PhD}$ thesis aims at investigating advanced technologies to optimize the LDM performance. The potential conjunction with other disruptive techniques, such as Multi-Radio Frequency Channel (MultiRF) technologies, or co-located MIMO transmissions are needed. Another important research field is the the SIC process, and how it can be modified for increasing the overall performance with a reduced complexity. 


\subsection{Objectives and Scope}

This $\mathrm{PhD}$ thesis will investigate and assess the novel transmission technique known as LDM with the goal of maximizing the spectral efficiency and signal robustness of DTT systems to provide mobile and fixed services simultaneously in the same RF channel.

The main objective of this dissertation is to evaluate and optimize the joint performance of LDM with other advanced signal processing techniques, in order to maximize the overall system performance. The partial objectives of the dissertation are:

- To propose optimized demapping algorithms that increase the performance of the two LDM layers. The commonly used demapping approach is to consider the lower layers below can be regarded as AWGN-like interference. However, potential gains can still be achieved if underlying layers are not considered AWGN. The new demapping approach for the upper layer considers the distribution of the symbols of the lower layer in the demapping processing. The proposed lower layer algorithm forwards the a-priori information obtained by the upper layer onto the lower layer demapping. The implementation of such algorithm may allow eliminating the need of the traditional SIC process.

- To discern the most suitable ATSC 3.0 pilot configuration for LDM, taking into account that is shared by both layers. The selection is more challenging than in DVB-T2. While $8 \mathrm{PP}$ with the same pilot boosting are allowed in DVB-T2, ATSC 3.0 offers up to 16 different $\mathrm{PP}$, where each one could use up to 5 different pilot boostings. Thus, not only a pilot density but also a pilot boosting trade-off should be bear in mind. Regarding pilot density, the selected PP must be sufficiently dense to follow channel variations in time and frequency domain, which is a fundamental aspect for the upper layer in mobile channels. However, the denser the PP, the lower the data rate, which is the main objective of the lower layer. Regarding pilot boosting, the channel accuracy is increased by using higher values. However, the higher the pilot boosting, the lower the available power for data transmission. The studies are done with different fading channels for mobile and fixed reception. The most suitable pilot configuration for the TDM mode of ATSC 3.0 is also provided.

- To evaluate the frequency diversity gains that CB technology can provide to LDM. CB enables services to exceed the data rate 
offered by a single RF channel. It also permits an improved RF performance by means of an inter-RF frequency interleaving across the RF channels. The combination of LDM and CB is allowed in ATSC 3.0, but it has never been evaluated, so that their joint performance is not known. First, the potential use cases of CB technology in ATSC 3.0 are evaluated looking for the finest system performance and the lowest complexity. The performance gains of the joint configurations are studied for the two LDM layers. In addition, the joint configuration with TFS, the MultiRF technology adopted in DVB-NGH, is also assessed.

- To determine the most suitable distributed MISO scheme for the joint transmission with LDM. Distributed MISO schemes are implemented for limitting SFN self-destructive interferences. ATSC 3.0 has adopted the frequency predistortion technology called TDCFS. It de-correlates the signals from transmitter, enhancing the frequency selectivity at receivers in order to prevent highly destructive cancellations at short echo delays. However, for medium and long echo delays, no performance gains are expected. They are introduced in such a way that special signal processing at the receivers is not necessary. Although the joint transmission of LDM and TDCFS is currently allowed by ATSC 3.0 standard, the joint performance has not been evaluated during the standardization process. In addition, the joint transmission of LDM with the well-known Alamouti scheme, adopted in DVB-T2 and DVB-NGH, which has not been assessed in the literature yet, is also evaluated. The performance of the different configurations is evaluated under different SFN scenarios.

- To evaluate the optimum combination of LDM with the colocated MIMO profile adopted in ATSC 3.0. The ATSC 3.0 standard has adopted both technologies separately, but no combination of them is planned yet. Although theoretical studies have been conducted, a more detailed study is required. A trade-off analysis between complexity constraints and performance benefits emerges. The first combination that may be extracted is the use of MIMO for the two LDM layers. It will increase the capacity and reliability of the two services. Nevertheless, it will highly increase receivers complexity and in turn, device's cost. Therefore, a less complex multi-antenna scheme can be more advisable for the top-layer, since it is oriented for low-complex mobile receivers. An study of channel estimator's memory requirement is particularly necessary, as new MIMO PP are required. The top-layer and lower layer performance is evaluated in mobility and fixed-rooftop conditions for receivers with 
one and two antennas, and with the two MIMO Pilot (MP) encodings (Walsh-Hadamard (WH) and Null Pilot (NP)) adopted in ATSC 3.0.

\subsection{State-of-the-Art}

\section{Superposition Coding}

In 1972-74, Cover and Bergmans [47], [48] showed that Superposition Coding (SCM) dominated asymptotically FDM, which in turn dominated TDM, in the presence of AWGN channel. This gain increased with the SNR gap of the superimposed signals due to the UEP ratio. In order to prove this statement, they made use of the Shannon capacity expression [8]. Considering the capacity of a channel operating as:

$$
C=W \ln \left(1+\frac{P}{N W}\right)
$$

where $N$ is the noise power spectral density, $P$ is the available power and $W$ is the total bandwidth. Whereas the set of achievable rates $\left(R_{1}, R_{2}\right)$ for what they referred as "naive time-sharing" were given by:

$$
\left\{\begin{array}{l}
R_{1}=\tau_{1} W \ln \left(1+\frac{P}{N W}\right) \\
R_{2}=\tau_{2} W \ln \left(1+\frac{P}{N W}\right)
\end{array}\right.
$$

where $\tau_{2}=1-\tau_{1}$ were the proportion of the transmitted time, or for FDM by:

$$
\left\{\begin{array}{l}
R_{1}=W_{1} \ln \left(1+\frac{P}{N W}\right) \\
R_{2}=W_{2} \ln \left(1+\frac{P}{N W}\right)
\end{array}\right.
$$

where $W_{1}+W_{2}=W$ were the bandwidth portions of transmitter $i, i=1,2$, the achievable rates for SCM yielded:

$$
\left\{\begin{array}{l}
R_{1}=W \ln \left(1+\frac{P_{1}}{N W}\right) \\
R_{2}=W \ln \left(1+\frac{P_{2}}{N W+P_{1}}\right)
\end{array}\right.
$$

where $P_{1}+P_{2}=P$ are the allocated power to each stream. Taking into account this equations, the achievable rates of the multiplexing schemes, it is clearly shown that SCM outperformed TDM, as it is illustrated in Figure 1.2. 


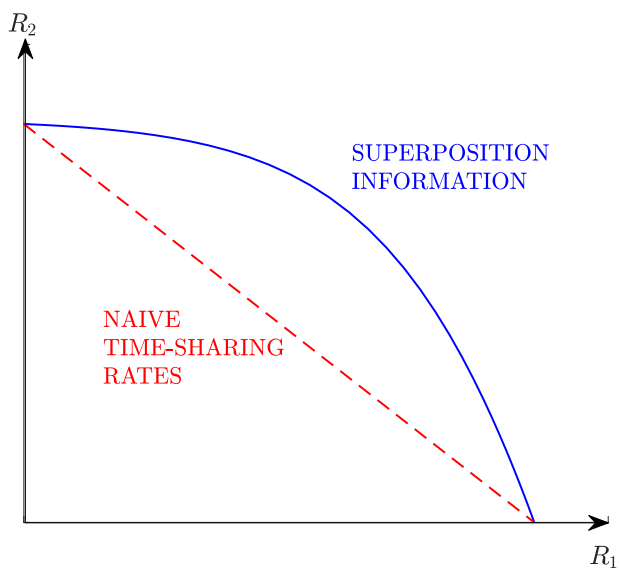

Figure 1.2: Rates achievable by naive time sharing, fixed frequency division and SCM [47].

However, Gadkari and Rose [53] proved in 1999 that this statement was only valid under ideal channel coding. For practical channel codes, the validity of previous results depended on the UEP ratio between the superimposed streams. Because of this, and because of the required receiver complexity that the SIC process entailed, SCM was not considered for any wireless communication system until LDM in ATSC 3.0, and NOMA in LTE.

\section{Cloud Transmission}

In 2012, Wu et al. proposed a new system to use the spectrum more efficiently [49]. The system was called Cloud Transmission (CloudTxn). The main CloudTxn trait was to deploy DTT networks with frequency reuse 1, i.e. all RF channels can be transmitted from every transmitter. This system would require of ultra-robust transmission modes for facing-off co-channel interferences. It can be seen in Figure 1.3 that with CloudTxn, only one RF channel was needed to cover the same area as four RF channels in a conventional Multiple Frequency Network (MFN) (frequency reuse 4).

It was established that each service should be able to support a negative AWGN SNR threshold of $-3 \mathrm{~dB}$. Thus, it should make use of the advanced techniques in channel coding, such as LDPC. If the AWGN SNR threshold was of $-3 \mathrm{~dB}$, the CloudTxn system was able to tolerate a $0 \mathrm{~dB}$ noise along with a $0 \mathrm{~dB}$ co-channel interference. However, this very robust signal provided in turn a very low data rate. In order to increase the system data rate, they proposed to transmit two 

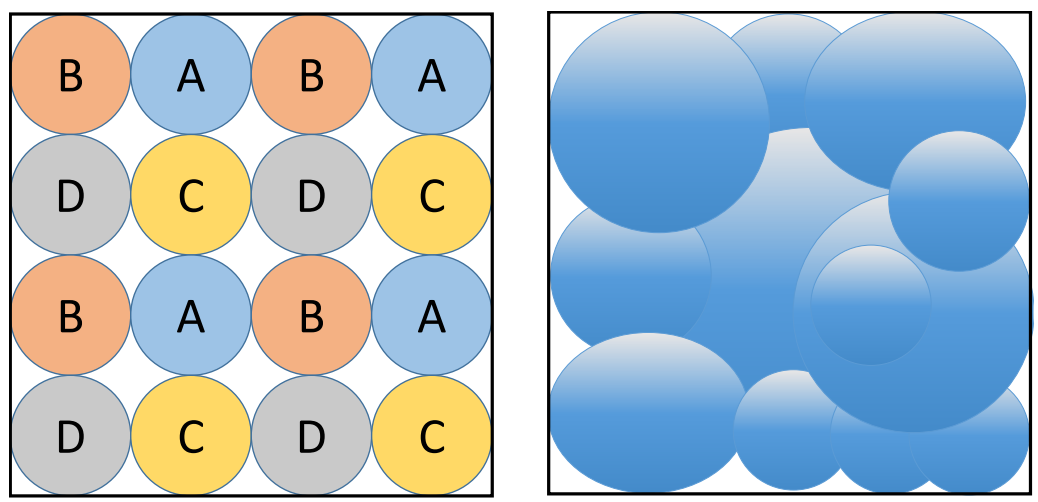

Figure 1.3: Left: MFN coverage with 4 RF channels. Right: CloudTxn coverage with 1 RF channel [49].

signals from the same station and making use of a hierarchical spectrum re-use technique. Thanks to the high degree of robustness of the CloudTxn signal, a second signal could be inserted in the same RF channel with an injected power level. Thus, the combined system spectrum efficiency could be higher than in conventional multiplexing techniques due to the UEP ratio benefits demonstrated since 1972. At the receiver, the CloudTxn signal was first decoded. The second signal was obtained by subtracting the decoded CloudTxn signal from the combined signal, i.e. by means of SIC [54]. The CloudTxn system was submitted for the ATSC 3.0 physical layer call for proposals. However, further studies were needed, especially regarding the transmitter identification, synchronization and signal cancellation techniques.

\section{Layered Division Multiplexing}

The originally introduced CloudTxn system was adopted in ATSC 3.0 as LDM. Aiming at reducing receivers' complexity some variations from the original proposal were introduced. Whereas the CloudTxn proposal combined two independent signals, i.e. with independent TIL, Frequency Interleaver (FIL), PP, FFT size, and GI duration, the adopted LDM signal was formed by two-layers, Core Layer (CL) and Enhanced Layer (EL), which are configured with different Bit-Interleaved Coded Modulation (BICM) chains but share the rest of physical layer blocks. Hence, the multiplexed layers are still configured with different transmission modes, but the SIC can be developed with low complexity. 


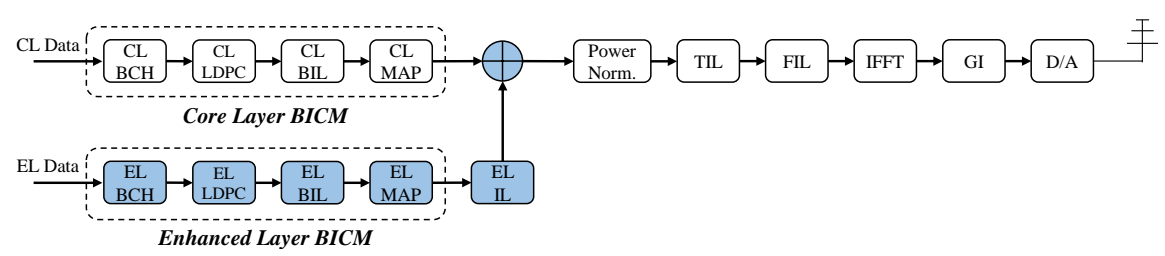

(a) LDM transmitter block diagram

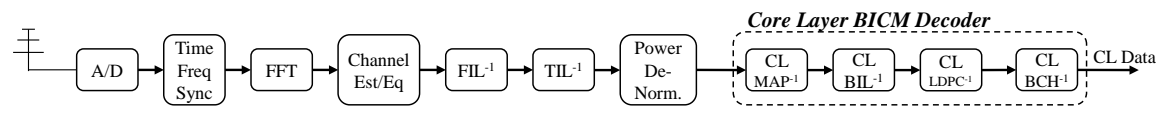

(b) LDM mobile receiver block diagram

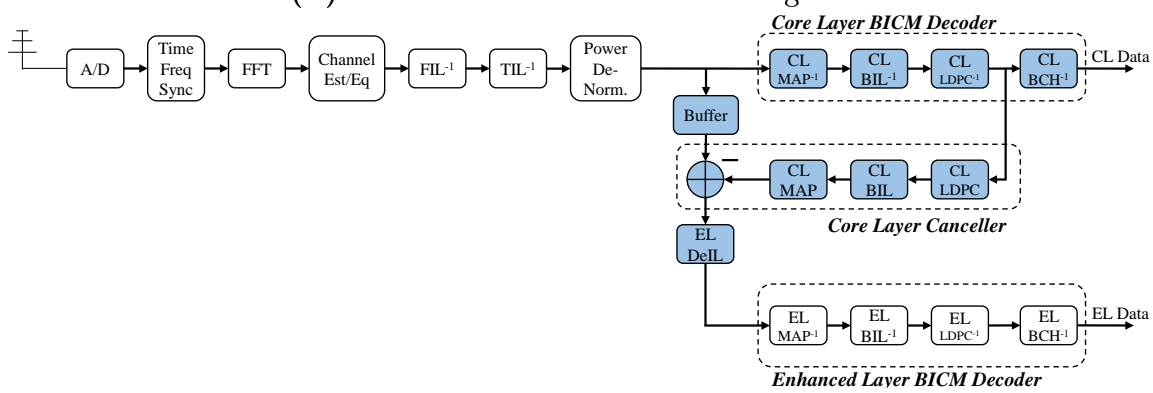

(c) LDM fixed receiver block diagram

Figure 1.4: LDM transmitter, mobile receiver, and fixed receiver block diagrams. Bluecoloured blocks represent additional complexity compared to a single layer system [51].

Figure 1.4 depicts the ATSC 3.0 transmitter and receiver block diagrams with LDM technique. The additional blocks that an LDM transmission requires are blue-coloured. At the transmitter it can be observed that the two layers are combined symbol by symbol. Hence, the additional LDM blocks at transmitter, compared to a single-layer transmitter, are the EL BICM, the Injection Level $(\Delta)$ controller, and the Power Normalizer. Since the CL and EL share the same TIL, and FIL, as well as the PP, the MISO algorithm, the FFT, and the GI, there should be no synchronization problems between layers at receiver side.

LDM receivers are classified according to the layer to be demodulated. On the one hand, mobile receivers, which are only intended to decode the CL, treats the EL as additional noise. Thus, there is no complexity increase compared to single layer receivers. On the other hand, fixed receivers, intended to decode the EL increases their complexity with respect to single layer receivers. They should first decode the CL, which is subsequently regenerated and can- 
Table 1.3: LDM gain over TDM in $0 \mathrm{~dB}$ echo (90\% GI) and TU-6 channels [51].

\begin{tabular}{cccc}
\hline \hline Mobile Mode & $\begin{array}{c}\text { Mobile 55\% } \\
\text { Time }\end{array}$ & $\begin{array}{c}\text { Mobile 40\% } \\
\text { Time }\end{array}$ & $\begin{array}{c}\text { Mobile 30\% } \\
\text { Time }\end{array}$ \\
\hline 2.0 Mbps & $1.4 \mathrm{~dB}$ & $3.3 \mathrm{~dB}$ & $7.0 \mathrm{~dB}$ \\
\hline $2.7 \mathrm{Mbps}$ & $0.9 \mathrm{~dB}$ & $4.6 \mathrm{~dB}$ & $5.2 \mathrm{~dB}$ \\
\hline 4.1 Mbps & $-1.1 \mathrm{~dB}$ & $1.5 \mathrm{~dB}$ & $6.1 \mathrm{~dB}$ \\
\hline \multirow{2}{*}{ Fixed Mode } & Fixed 45\% & Fixed 60\% & Fixed 70\% \\
& Time & Time & Time \\
\hline 14.3 Mbps & $5.8 \mathrm{~dB}$ & $1.2 \mathrm{~dB}$ & $-0.4 \mathrm{~dB}$ \\
\hline 20.5 Mbps & $7.9 \mathrm{~dB}$ & $3.0 \mathrm{~dB}$ & $-1.1 \mathrm{~dB}$ \\
\hline 24.6 Mbps & $\mathrm{N} / \mathrm{A}$ & $3.8 \mathrm{~dB}$ & $0.8 \mathrm{~dB}$ \\
\hline 30.1 Mbps & N/A & N/A & $1.7 \mathrm{~dB}$ \\
\hline \hline
\end{tabular}

celled. Therefore, a memory buffer to store the combined signal at the time de-interleaving output should be used. In [51], considering the adopted LDM system in ATSC 3.0, the fixed receivers memory increase for the worst case was estimated to $12 \%$.

As it has been shown, the complexity increase of the LDM system is almost negligible thanks to share all the OFDM waveform parameters. However, there is still a trade-off between the optimum PP, FFT, and GI configuration for delivering mobile and fixed services. Taking this constraint into account, very robust LDPC codes were adopted in ATSC 3.0 [55]. In fact, it was demonstrated in [56], that taking advantage of these error correction techniques, it was feasible to use a large FFT size (wanted by the EL) on mobile channels (planned for the CL). Moreover, in [57] it was shown that the speed limits of LDM systems are significantly higher than those for TDM systems, and even it was possible to deliver both CL and EL using the more efficient FFT 32k size. As a summary regarding performance simulations, Table 1.3, extracted from [51], show the LDM gains over TDM in realistic scenarios and taking into account the lack of OFDM flexibility of LDM versus TDM. It was observed that LDM had always from $3 \mathrm{~dB}$ to $9 \mathrm{~dB}$ gains over TDM. However, the PP performance in an LDM system has not been investigated yet.

All the previous simulation results were also corroborated by laboratory tests using an Software Defined Radio (SDR) platform [58], [59]. Furthermore, field 
test results carried out in Jeju city, South Korea [60]-[62] or in Cleveland, U.S. [63] verified that LDM enabled broadcasters to simultaneously provide a variety of services with different robustness within a single RF channel more efficiently. It should not be forgotten that in case a broadcaster is still eager to transmit via TDM, it is not incompatible with LDM and both multiplexing modes can be jointly used, as it was described in [64], [65].

Finally, although LDM is seen as an efficient multiplexing mode for the simultaneous delivery of mobile and fixed services within a single RF channel, there are other use cases where it can be employed. An alternative LDM use case is the seamless local service insertion in SFN environments proposed in [66]. In this study, the CL was used to deliver services operating in SFN, while the EL (in this case with a negative SNR threshold) could provide seamless local service from each SFN transmitter without coverage gaps among SFN transmitter service areas. Another LDM use case is an efficient transmission method based on Scalable HEVC (SHVC) technology, which was proposed in [67]. In this combination, the CL was assigned to the SHVC Base Layer, while the EL was assumed for the SHVC Enhancement Layer. It was shown that for the SHVC 0.33 layers ratio, which provided the maximum performance in the SHVC perspective, the overall gain of the LDM and SHVC combination was further improved.

\subsection{Thesis Outline and Contributions}

This thesis is presented by compendium of publications, so it meets the structure regulation (article 11 point 4-e) from Universitat Politècnica de València (UPV). The thesis has the following structure: Chapter 1 introduces the research topic as well as the motivations and objectives of its evaluation. Section 1.6 provides a complete list of contributions originated during the thesis work. Chapters 2, 3, 4, 5 and 6 correspond to the submitted publications, adopted to the format of the thesis. A general discussion of the results extracted from the publications is analyzed in Chapter 7. Finally, Chapter 8 outlines the main conclusions of this dissertation, and presents future research topics associated with the results from this work. The methodology approach is partially covered in each of the main chapters of the dissertation. The key contributions of the core chapters of this thesis are the following: 


\section{Chapter 2: Information-Theoretic Analysis and Performance Evaluation of Optimal Demappers for Multi-Layer Broadcast Systems}

Whereas the low-complexity implementation traditionally assumed in the literature for the CL demapping assumes the EL as an additional AWGN [51], an optimized demapping algorithm, which is aware of the EL constellation shape is proposed and analyzed. Moreover, a new EL demapping algorithm that may allow eliminating the need of the traditional SIC process performed in LDM [51] is also proposed. The new approach forwards the a-priori information obtained by the CL to the EL. The different demapping alternatives are evaluated from a generic point of view, via information theory. The complexity in number of distances to be computed by each alternative is also studied. A second part evaluates, by means of physical layer simulations, the performance of the two new demapping alternatives for a wide range of Modulation and Coding Rate (MODCOD) and Injection Level $(\Delta)$ and are compared with the approaches commonly used. For the CL demapping, two different channel models are considered: AWGN and independent and identically distributed (i.i.d.) Rayleigh. The EL demapping performance is evaluated with the AWGN and DVB Fixed Rice (DVB-F1) channels [68]. Publication [J3] from Section 1.6 is derived from this chapter.

\section{Chapter 3: Scattered Pilot Performance and Optimization for ATSC 3.0}

This chapter provides the performance evaluation in terms of Bit Error Rate (BER) and Mean Square Error (MSE) of the different pilot configurations allowed in ATSC 3.0. Different studies have shown the impact of other common waveform parameters, such as the TIL, FFT, and GI [56], [59]. However, the impact of Scattered Pilot (SP) was not investigated so far. Considering that both LDM layers share the same pilot configuration, there is a trade-off between a high pilot density (required by the CL) versus a low pilot overhead (required by the EL).

The first part provides the optimum SP density and SP boosting for mobile and fixed reception conditions separately, i.e., in TDM systems. But LDM requires to share the SP for the two layers. Thus, there is a trade-off for the optimum SP configuration between the a high pilot density for the mobile layer versus a low pilot overhead for the fixed layer. The second contribution takes into account this compromise and provides an optimum configuration. The last contribution aims at evaluating the channel estimation accuracy due to 
the Cross-Layer Interference (CLI), which is related with a forwarded error from the CL cancellation process.

Publications [C2] and [J2] from Section 1.6 are obtained as a result of the investigations from this chapter

\section{Chapter 4: Layered Division Multiplexing with Multi-Radio Frequency Channel Technologies}

The work presented in this chapter investigates the joint implementation of LDM with CB and with TFS. It should be highlighted that LDM and CB have been adopted for the first time in a terrestrial broadcasting standard. Hence, their joint performance is not known.

At first, the potential use cases and the related advantages are highlighted. From the different alternatives, the most complete combination is the transmission of CB using the SNR averaging mode on both LDM layers. It would allow to take advantage the three main gains from MultiRF techniques on both layers: doubling the service data rate, exploiting Statistical Multiplexing (StatMux) capabilities, and improving transmission robustness by inter-RF frequency interleaving.

Next, the requirements for the joint implementation with CB or with TFS are investigated. It is observed that $\mathrm{CB}$ does not imply additional requirements, beyond ensuring the proper exchange between cells by the Cell Exchanger block. On the other hand, additional requirements are observed if TFS is used. In particular, the distribution of the service across the multiple RF channels is done by the TIL. Thus, the impact on the even distribution, depending on the TIL scheme used is investigated. Regarding receiver aspects, the critical point is the TFS tuning operation, where a proper scheduling is needed to take into account the frequency hopping time.

Last, the coverage gains for the CL and for the EL are evaluated in mobile and fixed reception conditions, respectively. The performance is evaluated by means of physical layer simulations and compared with the transmission over the RF channel with the worst SNR condition, i.e. the one that would be limiting the reception of the service in a classical transmission. Since the even distribution of TFS is obtained by the TIL, different TIL schemes and TIL lengths are considered. In addition, different correlation factors among the channel realizations, receiver speeds, and number of RF channels are also analyzed. 
Publications [C1] and [J1], listed in Section 1.6, are derived from this chapter.

\section{Chapter 5: Layered Division Multiplexing with Distributed Multiple-Input Single-Output Schemes}

This chapter investigates the joint implementation of LDM with distributed MISO schemes, which are commonly used in DTT systems to limit SFN destructive interferences [69]. The distributed MISO algorithm adopted in ATSC 3.0 is considered, TDCFS. In addition, the potential introduction of MISO Alamouti in future ATSC 3.0 releases is evaluated, and compared with TDCFS. The investigations of this chapter resulted in publication [J4] from Section 1.6.

The first part analyzes the joint implementation aspects at transmitter and receiver sides. It is observed that the joint implementation with TDCFS is easier to be implemented, as the filtering process is introduced in a way that special signal processing is not necessary at receivers. On the other hand, Alamouti requires the use of orthogonal PP among transmitters, and a more complex channel estimator and equalizer at receivers.

The second part evaluates the performance of the two LDM layers with Alamouti and TDCFS for different SFN scenarios (with different echo delays and number of transmitters) under real channel estimations. Whereas Typical Urban (TU-6) realizations are assumed for mobility conditions in the CL evaluation, uncorrelated realizations for modeling fixed-rooftop reception conditions are employed in the EL evaluation.

\section{Chapter 6: Layered Division Multiplexing with Co-Located Multiple-Input Multiple-Output Schemes}

The joint transmission of LDM with co-located MIMO schemes is assessed in this chapter. The multiple antenna schemes have been adopted in ATSC 3.0 in order to improve the quality and reliability of a transmitted service without additional bandwidth or transmission power [34]. Nevertheless, it requires a more complex transmitter and receiver. Despite ATSC 3.0 has adopted LDM and co-located MIMO technologies separately, a joint transmission is not contemplated yet. A trade-off between complexity and performance is sprung up.

Two primary use cases are come up, depending on the complexity allowed in mobile receivers. A first use case employs only one antenna at mobile receivers. 
It is able to exploit spatial diversity on the CL and spatial multiplexing on the EL, without an excessive extra cost on mobile receivers. For the second use case, it is considered that both receivers (mobile for the CL and fixed for the EL) are implemented with two antennas. It exploits spatial diversity as well as spatial multiplexing on both LDM layers at the expense of more complex mobile receivers. In addition, the two primary use cases are divided in turn into two secondary use cases, whether the TDCFS filtering is enabled or disabled.

The implementation aspects of the two proposed joint use cases is next analyzed. It is realized that apart from the additional requirements of LDM and MIMO by themselves, the joint transmission does not put in additional constraints. Because of the mandatory use of MP encodings for the EL and MIMO transmission, special attention to channel estimator requirements is paid.

Physical layer performance of the all the use cases is finally evaluated. The performance evaluation of the two LDM layers is assessed for different MIMO channel models and with the two MP encodings. The submitted publications [C4] and [J5], listed in Section 1.6, are derived from this chapter. 


\subsection{List of Contributions}

\section{International Journals}

J1 E. Garro, J.J. Gimenez, S.I. Park, and D. Gomez-Barquero, "Layered Division Multiplexing with Multi-Radio Frequency Channel Technologies," IEEE Trans. Broadcast., vol. 62, no. 2, pp. 365-374, June 2016

J2 E. Garro, J.J. Gimenez, S.I. Park, and D. Gomez-Barquero, "Scattered Pilot Performance and Optimization for ATSC 3.0," IEEE Trans. Broadcast., vol. 63, no. 1, pp. 282-292, March 2017

J3 E. Garro, J.J. Gimenez, P. Klenner, and D. Gomez-Barquero, "Information-Theoretic Analysis and Performance Evaluation of Optimal Demappers for Multi-Layer Broadcast Systems," IEEE Trans. Broadcast., in press 2018

J4 E. Garro, C. Barjau, D. Gomez-Barquero, J. Kim, S. I. Park, and N. Hur, "Layered Division Multiplexing with Distributed Multiple-Input SingleOutput Schemes," IEEE Trans. Broadcast., in press 2018

J5 E. Garro, C. Barjau, D. Gomez-Barquero, J. Kim, S.I. Park, and H. Hur, "Layered Division Multipexing with Co-Located Multiple-Input MultipleOutput Schemes," to be submitted to IEEE Trans. Broadcast.

J6 T. Shitomi, E. Garro, K. Murayama, D. Gomez-Barquero, "MIMO Scattered Pilot Performance and Optimization for ATSC 3.0," IEEE Trans. Broadcast., in press 2018

\section{International Conferences}

C1 E. Garro, J.J. Gimenez, D. Gomez-Barquero, and S.I. Park, "Performance Evaluation of Layer Division Multiplexing (LDM) combined with Time Frequency Slicing (TFS)," 2015 IEEE International Symposium on Broadband Multimedia Systems and Broadcasting, Ghent, Belgium, June 2015.

C2 E. Garro, J.J. Gimenez, D. Gomez-Barquero, and S.I. Park, "Pilot Optimization for Mobile Services in ATSC 3.0," 2016 IEEE International Symposium on Broadband Multimedia Systems and Broadcasting, Nara, Japan, June 2016. 
C3 T. Shitomi, E. Garro, K. Murayama, and D. Gomez-Barquero, "Performance Evaluation of MIMO Channel Estimation for ATSC 3.0," 2017 IEEE International Symposium on Broadband Multimedia Systems and Broadcasting, Cagliari, Italy, June 2017.

C4 E. Garro, C. Barjau, D. Gomez-Barquero, J. Kim, and S.I. Park, "Study on the Optimum Co-Located MIMO scheme for LDM in ATSC 3.0: Use Cases and Core Layer Performance," 2018 IEEE International Symposium on Broadband Multimedia Systems and Broadcasting, Valencia, Spain, June 2018.

\section{Patents}

P1 S.I. Park, J.Y. Lee, S. Kwon, H.M. Kim, B.M. Lim, D. Gomez-Barquero, E. Garro, J.J. Gimenez Apparatus for transmitting/receiving broadcasting signal using combination mode of layered division multiplexing and channel bonding, and method thereof, Application number: 15/212109 Publication number: US 2017/0019207

\section{Research Projects}

RP1 Tecnologías de Radiodifusión Digital Extra-Terrestres (ET-Broadcast) (TEC2014-56483-R-AR).

- Funding institution: Ministerio de Economiía, Industria y Competividad, Spain. Co-funded by the European FEDER found.

- Start date: $01 / 01 / 2015$

- Duration: 36 months

RP2 Time Frequency Slicing for Cloud Transmission - Implementation and Performance Evaluation Studies.

- Funding institution: Electronics and Telecommunications Research Institute, South Korea, grant number R0101-15-294

- Start date: $01 / 07 / 2014$

- Duration: 7 months 
RP3 Optimization and Performance Evaluation of LDM for ATSC 3.0.

- Funding institution: Electronics and Telecommunications Research Institute, South Korea, grant number R0101-15-294

- Start date: $01 / 06 / 2015$

- Duration: 8 months

RP4 MIMO for LDM.

- Funding institution: Electronics and Telecommunications Research Institute, South Korea, grant number R0101-15-294

- Start date: 01/06/2016

- Duration: 8 months

RP5 Multiple Antenna Schemes for LDM - Phase II.

- Funding institution: Electronics and Telecommunications Research Institute, South Korea, grant number 2017-0-00081

- Start date: $01 / 04 / 2017$

- Duration: 8 months

\section{International Research Visits}

V1 Electronics and Telecommunications Research Institute, Daejeon (South Korea). February - April 2016 (3 months).

\section{Standardization Activities}

ST1 2 contributions to the TG3/S32-3 Ad-hoc group on Waveform in the standardization process of ATSC 3.0.

ST2 2 contributions to the ATSC Recommended Practice - Guidelines for the Physical Layer Protocol. 



\section{Information-Theoretic Analysis and Performance Evaluation of Optimal Demappers for Multi-Layer Broadcast Systems}

E. Garro, J.J. Gimenez, P. Klenner, IEEE Trans. on Broadcast., in press 2018. Multi-layer broadcast systems distribute services across time and frequency domain by means of power-division multiplexing. Successive interference cancellation is required, in general, in order to extract the content of all services. For a low-complexity implementation, the receiver can obtain the strongest (top-layer) signal assuming underlying signals to behave like thermal noise. The thermal noise assumption may not be valid under certain conditions and a more accurate characterization of the interference could bring improved performance. This paper analyzes the validity of the noise-like assumption considering the power ratio between signals and the required Signal-to-Noise ratio (SNR) for error-free reception. The main contribution of the paper is the proposal of a demapping algorithm that exploits the knowledge of the constellation of underlying signals. Generalized Mutual Information, performance evaluation, and complexity analysis are provided with the AWGN-like assumptions and with the proposed alternative in order to assess the potential performance improvements that can be achieved. 

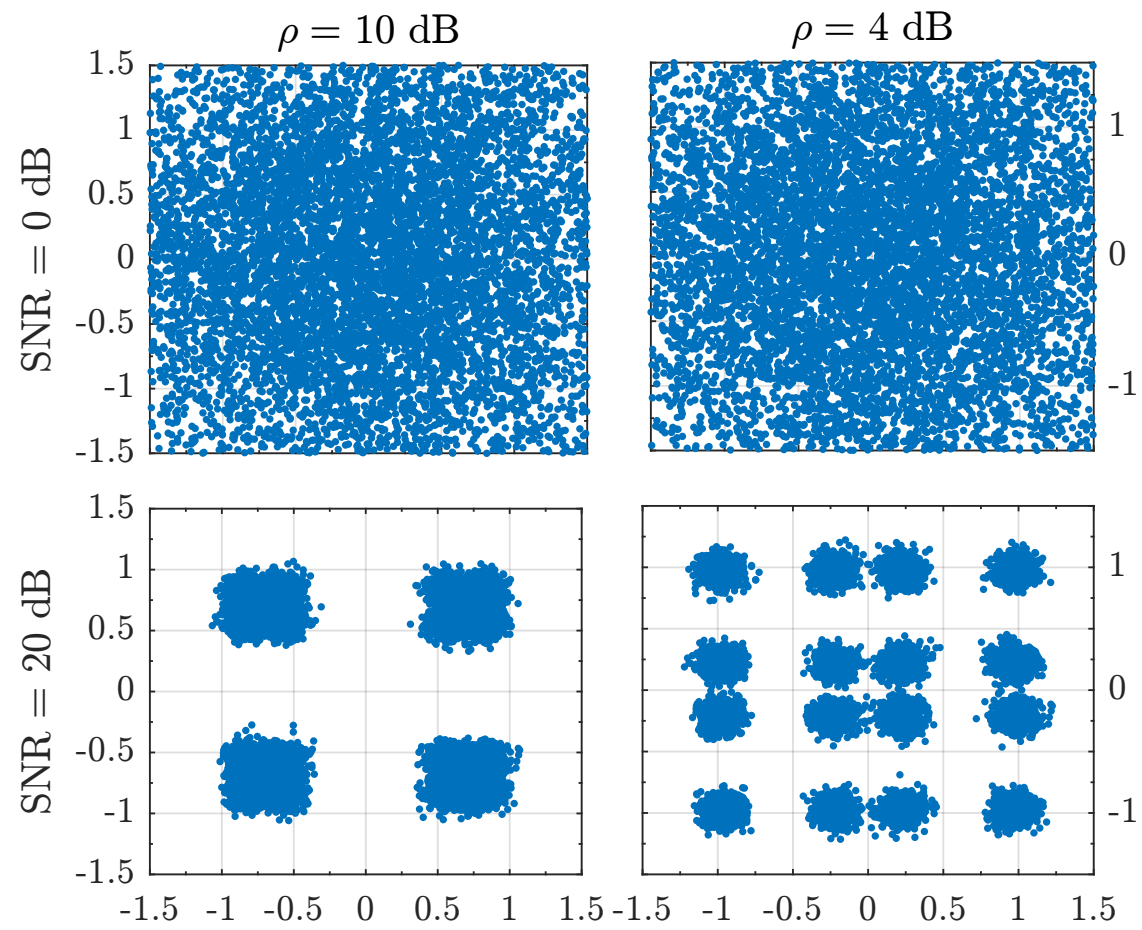

Figure 2.1: QPSK + QPSK signal with $\rho=10 \mathrm{~dB}$ (left) and $\rho=4 \mathrm{~dB}$ (right). SNR $=0$ $\mathrm{dB}$ (top) and $\mathrm{SNR}=20 \mathrm{~dB}$ (bottom).

\section{$2.1 \quad$ Introduction}

Multi-layer transmission has been raised as a relevant broadcast technology where the multiplexing of services is performed in the power domain while using $100 \%$ of the frequency and time resources. Implemented as Layered Division Multiplexing (LDM) in ATSC - Third Generation (ATSC 3.0) [26], the signal consists of the superposition of two signals/layers with different power levels. Each layer, namely Core Layer (CL) and Enhanced Layer (EL), passes through a different Bit-Interleaved Coded Modulation (BICM) chain. This brings the possibility to assign different robustness/capacity characteristics to different services, and hence, to target different reception conditions simultaneously. Once encoded, the signals are aggregated with different power levels.

The concept behind multi-layer transmission has also been considered to allow for frequency reuse-1 networks with Cloud Transmission (CloudTxn) [49] 
and Wideband reuse-1 (WiB) [70]. In the WiB concept, all stations are assumed to transmit signals configured with a robust Modulation and Coding Rate (MODCOD) that would enable reception in a highly interference-limited situation. The received signal consists of the superposition of multiple signals of the same nature (like in LDM) with a different power level according to propagation conditions and transmit power.

Receivers are able to perform the demodulation of the top-layer signal as long as the received Signal-to-Interference-and-Noise Ratio (SINR) is larger than the operating SNR of the selected MODCOD. The other signals/layers can be demodulated by Successive Interference Cancellation (SIC) algorithms. In case of two signals, the power ratio between them can be modeled by means of an Injection Level $(\rho)$.

The commonly used demapping approach is to consider that signals/layers below the top-layer can be regarded as Additive White Gaussian Noise (AWGN) like interference [45], [53]. However, potential gains can still be achieved if underlying layers are not considered AWGN. Figure 2.1 illustrates the impact of $\rho$ and SNR conditions for a multi-layer signal constituted of two QPSK constellations. Top figures show the received constellation symbols in a low SNR region $(0 \mathrm{~dB})$, where AWGN dominates regardless of $\rho$. Lower figures show the symbols in a higher SNR region $(20 \mathrm{~dB})$. It can be noticed from lower figures that for $\rho=10 \mathrm{~dB}$, the QPSK symbols look like being affected by AWGN distribution. On the other hand, with $\rho=4 \mathrm{~dB}$, this assumption is not valid. The resulting constellation presents symbols that are a combination of the symbols of the different layers, each one affected by AWGN. Hence, potential performance gains may be achieved if this effect is considered.

This paper expands the initial studies in [37], [71] of a demapping approach for LDM systems in which the AWGN-like interference assumption may not be valid. This new demapping approach considers the distribution of the symbols of the underlying LDM layer when demapping the top-layer signal, which brings a potential gain under certain circumstances at the expense of additional complexity. In addition to [71], the paper evaluates the new demapping concept from a generic point of view, via information theory, and studies the complexity of its implementation at receivers. Furthermore, a new algorithm is proposed, which forwards the a-priori information obtained by the demapping of the top-layer signal to the lower-layer signal. The implementation of such algorithm may allow eliminating the need of the traditional cancellation process performed in multi-layer systems. 
The rest of the paper is structured as follows: Section 2.2 presents the formulation of the proposed demapping algorithms, as well as a complexity analysis in terms of number of operations. Section 2.3 evaluates and compares the Generalized Mutual Information (GMI) limits of the new approach with the AWGN assumption. Top-layer signal performance results for a wide range of MODCODs and $\rho$ values are shown and discussed in Section 2.4. Moreover, performance evaluation for the lower-layer signal is also studied. Finally, conclusions are drawn in Section 2.5.

\subsection{Constellation Demapper Alternatives in Multi-layer Systems}

The received signal, considering that the transmitted signal is composed of two signals/layers, can be modeled by the following expression:

$$
y=x \cdot h+w=\left(\tau \cdot x_{t}+\beta \cdot x_{b}\right) \cdot h+w
$$

where $x_{t}$ and $x_{b}$ denote the top-layer and bottom-layer transmitted complexvalued symbols, with $\tau=1 / \sqrt{1+g^{2}}$ and $\beta=g / \sqrt{1+g^{2}}$ amplitudes, respectively. $g=10^{-\frac{\rho}{20}}$ is the injection level expressed in linear units.

Considering that $x_{t}$ is the first signal to be demodulated, a straightforward approach is to consider $x_{b}$ as an interfering contribution, which can be regarded as AWGN (in the following, Gaussian Demapping) [51]. Alternatively, the Optimum Demapping approach considers $x_{b}$ as useful information by considering its symbol alphabet. Although the demodulation of the bottom-layer signal can be performed by a hard-interference cancellation of the reconstructed top-layer symbols [51], the new method can also be extended to allow for a soft-cancellation approach.

\subsubsection{Gaussian Demapping (GD) Apprach}

Top-layer signal $\left(x_{t}\right)$ demapping

The GD assumes the bottom-layer signal $\left(x_{b}\right)$ as additional source of AWGN (with zero mean and single-sided variance $\sigma_{g}^{2}=\beta^{2}$ ). 
Using soft-decision decoding, the Log-Likelihood Ratios (LLR), $\Lambda_{t}^{G D}\left(b_{i}\right)$, for each coded bit $b_{i}, i=1, \ldots, m$ is calculated as:

$$
\Lambda_{t}^{G D}\left(b_{i}\right) \triangleq \log \frac{p\left(b_{i}=1 \mid y, h\right)}{p\left(b_{i}=0 \mid y, h\right)}=\log \frac{\sum_{x_{t} \in \zeta_{i}^{1}} p\left(y \mid \tau x_{t}, h\right)}{\sum_{x_{t} \in \zeta_{i}^{0}} p\left(y \mid \tau x_{t}, h\right)}
$$

where $\zeta_{i}^{b}$ denotes the signal subset of constellation points of $x_{t}$ with the $i$ th bit being $b_{i} \in\{0,1\} . p\left(y \mid \tau x_{t}, h\right)$ is the conditional probability density function (pdf) [72] corresponding to the expression:

$$
p\left(y \mid \tau x_{t}, h\right)=\frac{1}{\pi\left(\sigma_{g}^{2}+\sigma_{\omega}^{2}\right)} \exp \left(-\frac{\left|y-h \tau x_{t}\right|^{2}}{\sigma_{g}^{2}+\sigma_{\omega}^{2}}\right)
$$

The obtained LLRs passed through the Low Density Parity Check (LDPC) decoder so that the estimated bits of the transmitted signal are obtained.

\section{Bottom-layer signal $\left(x_{b}\right)$ demapping}

To retrieve the bottom-layer signal, the $\Lambda_{t}^{G D}\left(b_{i}\right)$ are LDPC-decoded, remodulated and subtracted from the received signal $y$ of (2.1). This process is defined as Hard Cancellation (HC) method. The system model at this point is:

$$
\tilde{y}=y-\tau \hat{x}_{t} \cdot h=\beta \cdot x_{b} \cdot h+w
$$

where $\hat{x}_{t}$ are the re-encoded complex-valued symbols of the top-layer signal.

The LLRs of the bottom-layer signal $\Lambda_{b}^{G D}\left(b_{j}\right)$ for each coded bit $b_{j}$ are finally calculated as:

$$
\Lambda_{b}^{G D}\left(b_{j}\right)=\log \frac{\sum_{x_{b} \in \zeta_{j}^{1}} p\left(\tilde{y} \mid \beta x_{b}, h\right)}{\sum_{x_{b} \in \zeta_{j}^{0}} p\left(\tilde{y} \mid \beta x_{b}, h\right)}
$$

being the pdf $p\left(\tilde{y} \mid \beta x_{b}, h\right)$ defined as:

$$
p\left(\tilde{y} \mid \beta x_{b}, h\right)=\frac{1}{\pi \sigma_{\omega}^{2}} \exp \left(-\frac{\left|\tilde{y}-h \beta x_{b}\right|^{2}}{\sigma_{\omega}^{2}}\right)
$$




\subsubsection{Optimum Demapping (OD) Approach}

As depicted in Figure 2.1, the AWGN-like assumption of $x_{b}$ on the $x_{t}$ demodulation may not be sufficiently accurate in certain conditions. Furthermore, the potential bad estimation of $x_{t}$ could be forwarded to $x_{b}$, since the latter demodulation makes use of the former. Therefore, a new approach that increases $x_{t}$ and $x_{b}$ performances becomes imperative. This section extends the formulation presented in [37], [71] by providing the complete LLR and pdf equations for the implementation of the OD approach for the top-layer signal. For the bottom-layer signal, a Soft Cancellation (SC) demapping formulation is also provided considering a-priori LLR values from the top-layer signal.

\section{Top-layer signal $\left(x_{t}\right)$ demapping}

The proposed Optimum Demapping algorithm assumes the knowledge of the bottom-layer signal constellation $\left(x_{b}\right)$ when demapping the top-layer signal $\left(x_{t}\right)$. To do so, Euclidean distances for all combinations resulting from the sum of the constellations of the two layers are calculated. Assuming the received signal of (2.1), the top-layer signal LLR $\Lambda_{t}^{O D}\left(b_{i}\right)$ is calculated according to:

$$
\Lambda_{t}^{O D}\left(b_{i}\right)=\log \frac{\sum_{x_{t} \in \zeta_{i}^{1}} \sum_{x_{b}} p\left(y \mid \tau x_{t}, \beta x_{b}, h\right)}{\sum_{x_{t} \in \zeta_{i}^{0}} \sum_{x_{b}} p\left(y \mid \tau x_{t}, \beta x_{b}, h\right)}
$$

The second summation term involves all possible transmitted $x_{b}$ values for each transmitted $x_{t}$. The pdf $p\left(y \mid \tau x_{t}, \beta x_{b}, h\right)$ is modeled as:

$$
p\left(y \mid \tau x_{t}, \beta x_{b}, h\right)=\frac{1}{\pi \sigma_{\omega}^{2}} \exp \left(-\frac{\left|y-h\left(\tau x_{t}+\beta x_{b}\right)\right|^{2}}{\sigma_{\omega}^{2}}\right)
$$

\section{Bottom-layer signal $\left(x_{b}\right)$ demapping}

The same top-layer signal HC process as in Section 2.2.1 can be performed using the optimum LLR values estimated in (2.7). However, the top-layer signal remodulation and hard-cancellation processes may be omitted if $\Lambda_{t}^{O D}\left(b_{i}\right)$ is regarded as a-priori LLR values on a SC demapping of the bottom-layer signal.

The use of a-priori LLR values in iterative processing is a well-established topic in the field. For example, a similar algorithm was proposed in [73] in 
the context of iterative demapping for multilevel modulation. It has not been addressed extensively though with regards to broadcasting by means of superposition modulation, where the de-facto standard is set by hard-successive interference cancellation (cf. [51]).

The expression for obtaining the bottom-layer signal LLR $\Lambda_{b}^{O D}\left(b_{j}\right)$ can be written as:

$$
\Lambda_{b}^{O D}\left(b_{j}\right)=\log \frac{\sum_{x_{b} \in \zeta_{j}^{1}} \sum_{x_{t}} p\left(y \mid \tau x_{t}, \beta x_{b}, h\right) P\left(x_{t}\right)}{\sum_{x_{b} \in \zeta_{j}^{0}} \sum_{x_{t}} p\left(y \mid \tau x_{t}, \beta x_{b}, h\right) P\left(x_{t}\right)}
$$

It can be observed that same conditional pdf as (2.8) is used. However, since bottom-layer signal LLR $\Lambda_{b}^{O D}\left(b_{j}\right)$ are calculated now, all possible transmitted $x_{t}$ values are considered for each transmitted $x_{b}$. $P\left(x_{t}\right)$, which refers to the transmitted vector probability, can be developed as:

$$
P\left(x_{t}\right)=\prod_{i=1}^{m} P\left(b_{i}\right) \propto \prod_{i=1}^{m} \exp \left(b_{i} \Lambda_{t}^{O D}\left(b_{i}\right)\right)
$$

\subsubsection{Demappers Complexity}

The OD approach can potentially provide gains in high SNR regions at the expense of increased complexity. The complexity of the two demapping approaches, GD and OD, is computed in terms of required number of Euclidean distances.

The GD approach for the top-layer signal involves the calculation of the distances between the received signal $y$ and all possible transmitted symbols $x_{t}$, resulting in $2^{m_{t}}$ Euclidean distances, with $m_{t}$ the number of transmitted bits per symbol of the top-layer signal. If top-layer signal hard-cancellation is performed, the bottom-layer signal requires the calculation of the Euclidean distances between the cancelled $\tilde{y}$ and all possible transmitted symbols $x_{b}$, leading to $2^{m_{b}}$, where $m_{b}$ is the number of transmitted bits per symbol of the bottom-layer signal. Therefore, the total number of Euclidean distances to be computed for the two signals is $2^{m_{t}}+2^{m_{b}}$.

On the other hand, the OD demapping approach for the top-layer signal involves the calculation of the distances between the received signal $y$ and all possible $x_{t}$ and $x_{b}$ symbol combinations. This second approach requires $2^{m_{t}+m_{b}}$ Euclidean distances. For the bottom-layer signal, the same number of distances to be computed are needed, but taking into account the a-priori LLR values 
as in (2.10). Thus, $2^{m_{t}+m_{b}+1}$ Euclidean distances are needed for the two-signal demodulation with the OD approach.

Figure 2.2 shows the transmitted symbols that are taken into account for the top-layer signal LLR calculation of the encoded bit $b_{0}$ with the two demapping approaches. For simplicity, a QPSK + QPSK signal is assumed $\left(m_{t}=2\right.$ and $\left.m_{b}=2\right)$. Thin dots correspond to points in $\zeta_{0}^{0}$ while thick dots corresponds to points in $\zeta_{0}^{1}$. As it can be observed, the received symbol (asterisk) is closer to one of the OD constellation points (right) than to one of the GD (left). Hence, a better performance can be provided. On the contrary, whereas GD computes $2^{2}=4$ Euclidean distances for getting $\Lambda_{t}^{G D}\left(b_{0}\right)$, OD computes $2^{2+2}=16$ distances for $\Lambda_{t}^{O D}\left(b_{0}\right)$, which can be likened to a 16QAM constellation. In order to reduce the number of Euclidean distances to be computed by OD at the expense of a performance loss, a semi-optimized approach was evaluated in [74]. It was observed that by employing constellation orders lower than the current bottom-layer constellation on the $\Lambda_{t}^{O D}\left(b_{i}\right)$ computation, the performance was degraded at most by $0.4 \mathrm{~dB}$.

Overall, the GD approach provides a low-complexity demapper implementation, which results in the most practical implementation for systems in which the layer demapping results AWGN limited. The OD, with increased demapping complexity, may be appropriate when the layers involved in the demapping process are configured with low order constellations. The demapper based on a-priori LLR values results in the most complex demapper which practical implementation should be carefully evaluated.

\subsection{Information-Theoretical Analysis of BICM systems}

In order to compare the demapping approaches presented in Section 2.2 for the top-layer signal, an information-theoretical study is investigated in terms of the error exponent and GMI of a BICM decoder. Hence, the mismatch effect of GD is also spotlighted.

\subsubsection{Error Exponent Analysis}

In [75] Gallager derived an upper bound for the average error probability over a random code ensemble and showed that the bound depends on a parameter expediently called error exponent, which in turns depend on Gallager function. Gallager assumed a maximum likelihood decoder with matched pdfs, and showed that the derivative of the Gallager function yields the capacity. 

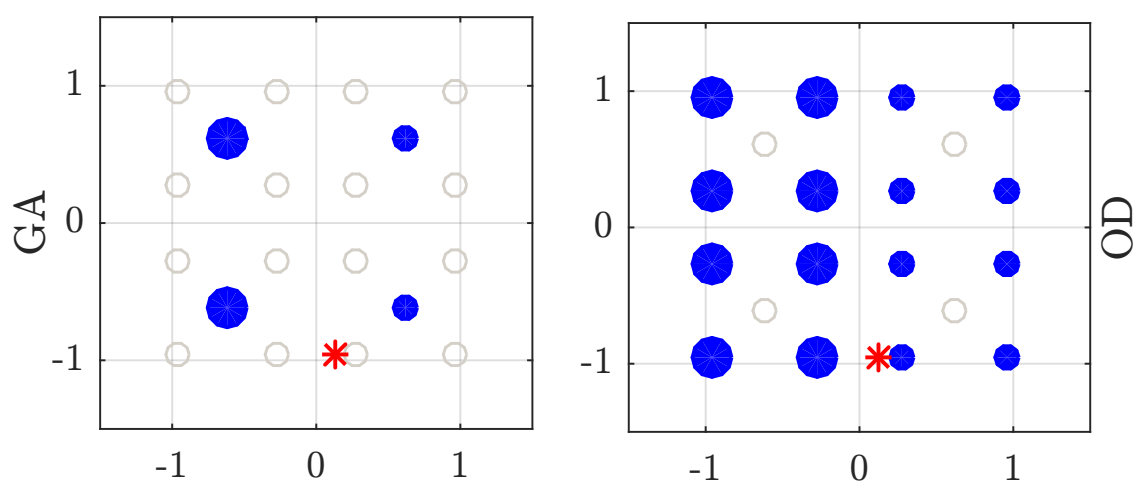

Figure 2.2: Transmitted points of the constellation (blue) for obtaining the LLR of the received symbol (red) with GD (left), and OD (right) approaches. Thin dots represent the points of $\zeta_{0}^{0}$ while thick dots represent the points of $\zeta_{0}^{1}$.

Gallager's derivation can be extended to consider mismatched decoding metrics (see [76] and the references therein). The average error probability over the code ensemble is then denoted by:

$$
\overline{P_{e}} \leq 2^{-N E_{\mathrm{r}}^{q}(R)}
$$

$N$ is the block length, and $E_{\mathrm{r}}^{q}(R)$ is the mismatched random coding error exponent, given by:

$$
E_{\mathrm{r}}^{q}(R)=\max _{0 \leq \varrho<1} \max _{s>0} E_{0}^{q}(\varrho, s)-\varrho R
$$

where $\varrho$ and $s$ are free parameters subject to optimization. $R$ denotes the coding rate.

For the specific case of BICM, the generalized Gallager function $E_{0}^{q}(\varrho, s)$ takes on the form

$$
E_{0}^{\mathrm{bicm}}(\varrho, s)=-\log _{2} \mathbb{E}\left\{\left(\frac{1}{2^{m}} \sum_{x^{\prime}} \prod_{i=1}^{m} \frac{q_{i}\left(b_{i}\left(x^{\prime}\right), Y\right)^{s}}{q_{i}\left(b_{i}(X), Y\right)^{s}}\right)^{\varrho}\right\}
$$

With a slight abuse of notation, the generic decoding metric for the $i$-th bit is given here by

$$
q_{i}\left(b_{i}(x)=b, y\right)=\sum_{x^{\prime} \in \zeta_{b}^{i}} p\left(y \mid x^{\prime}\right)
$$




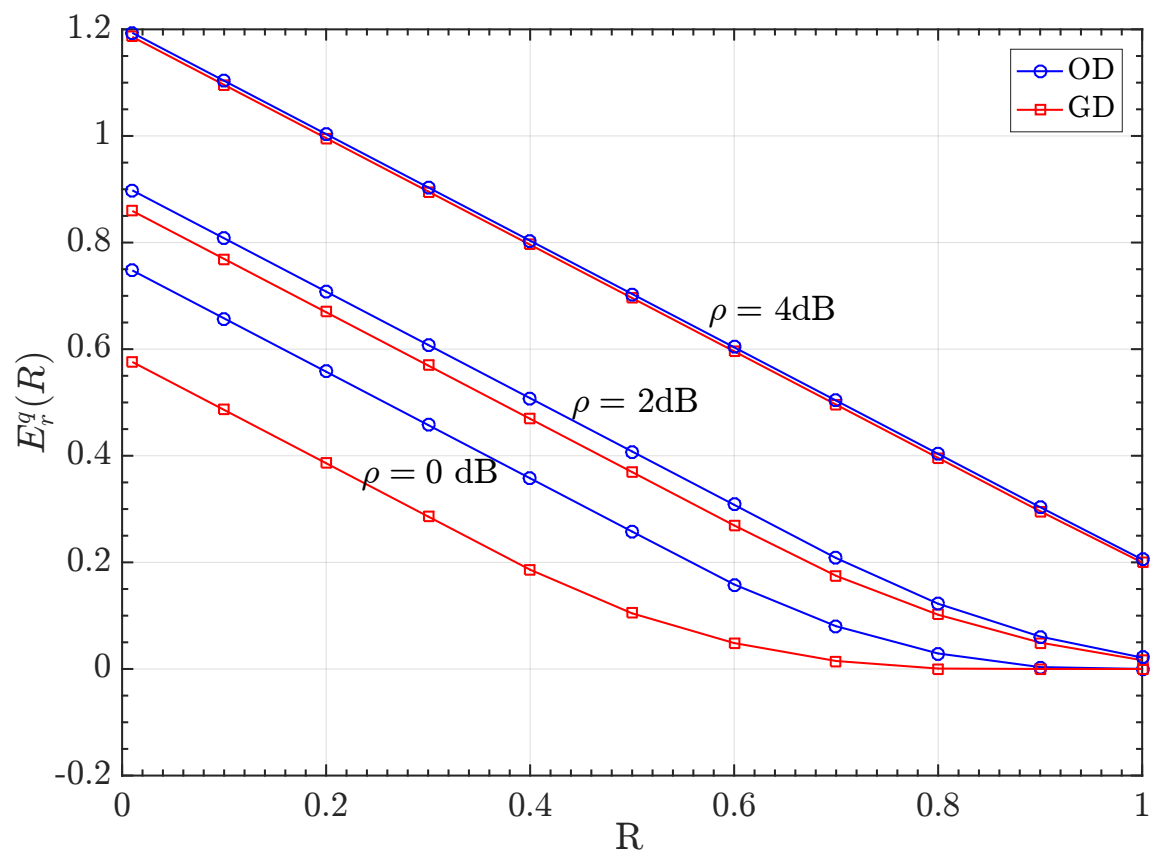

Figure 2.3: Error exponent for the top layer of QPSK + QPSK (relevant WiB case) with $\rho=0,2$, and $4 \mathrm{~dB}$, and $\mathrm{SNR}=10 \mathrm{~dB}$

where the transition probabilities $p\left(y \mid x^{\prime}\right)$ can be based on either matched or mismatched probabilities. The inverse mapping function $b_{i}(x)$ yields the $i$-th bit carried by symbol $x$.

As an example, the error exponent for OD and GD is shown in Figure 2.3 for top-layer signal employing QPSK with injection levels of $0 \mathrm{~dB}, 2 \mathrm{~dB}$, and 4 $\mathrm{dB}$ at an SNR of $10 \mathrm{~dB}$. The OD yields a larger error exponent over a wide range of code rate $R$ and thus, leads to a more robust system than GD for small injection levels. However, for larger injection levels, e.g., $4 \mathrm{~dB}$, the performance of GD and OD are on par. 


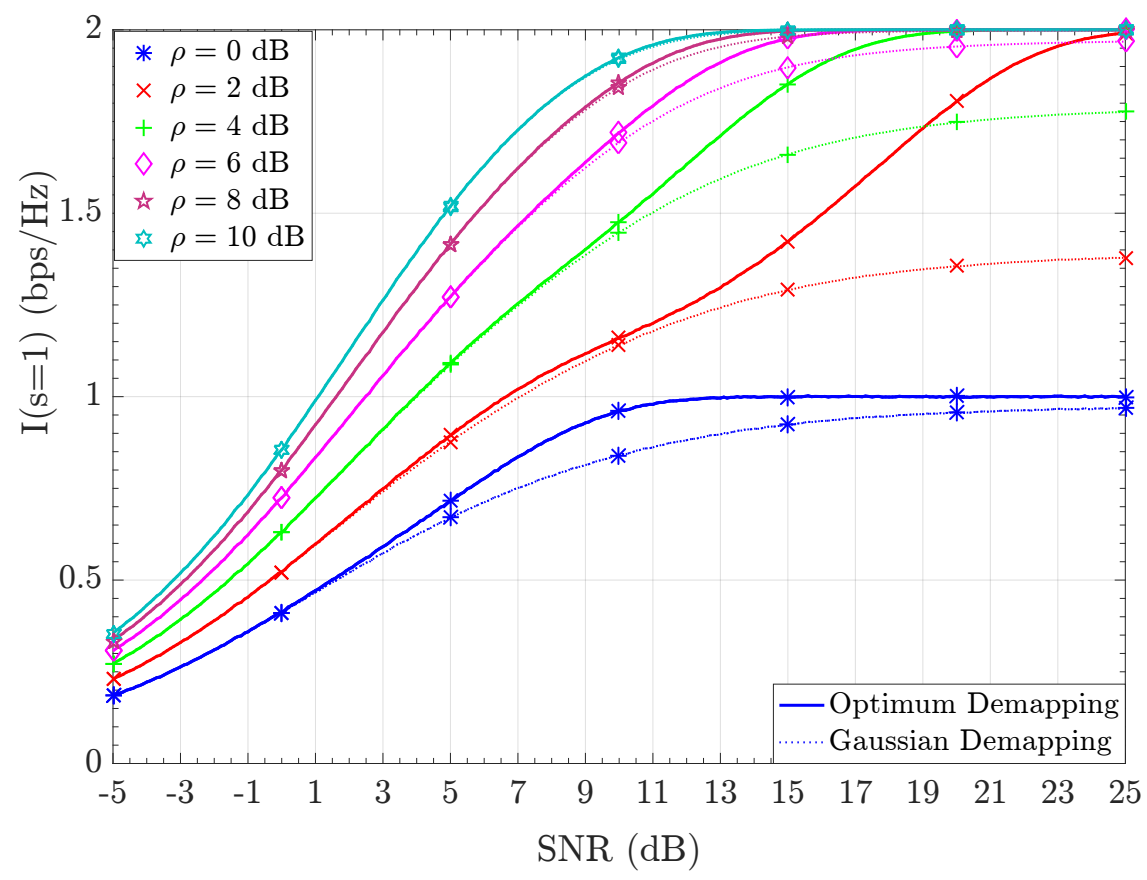

Figure 2.4: Top-layer signal $\left(x_{t}\right)$ QPSK + QPSK I-curves $(s=1)$ for different SNR. $\rho=0,2,4,6,8$, and $10 \mathrm{~dB}$.

\subsubsection{I-curves}

For any given constellation with spectral efficiency $m_{t}$, the I-curves determine the Forward Error Correction (FEC) code rate required to achieve error-free communication for a particular SNR [77]. The I-curves are obtained as the derivative of the Gallager function:

$$
\begin{aligned}
I(s) & =\left.\frac{d E_{0}^{q}(\varrho, s)}{d \varrho}\right|_{\varrho=0} \\
& =\sum_{i=1}^{m_{t}} \mathbb{E}\left\{\log _{2} \frac{q_{i}(b, y)^{s}}{\frac{1}{2} \sum_{b_{i}=0}^{1} q_{i}\left(b_{i}, y\right)^{s}}\right\}
\end{aligned}
$$

This section computes the I-curves of the top-layer signal bits with GD and OD by Monte Carlo simulations. For such purpose, Equation (2.15) is expressed in terms of LLRs and binary sign function $(\sigma(0)=-1$ and $\sigma(1)=1)$ by substituting $q_{i}\left(b_{i}, y\right)$ for $\exp \left(\frac{1}{2} \sigma\left(b_{i}\right) \Lambda_{t}\left(b_{i}\right)\right)$. 


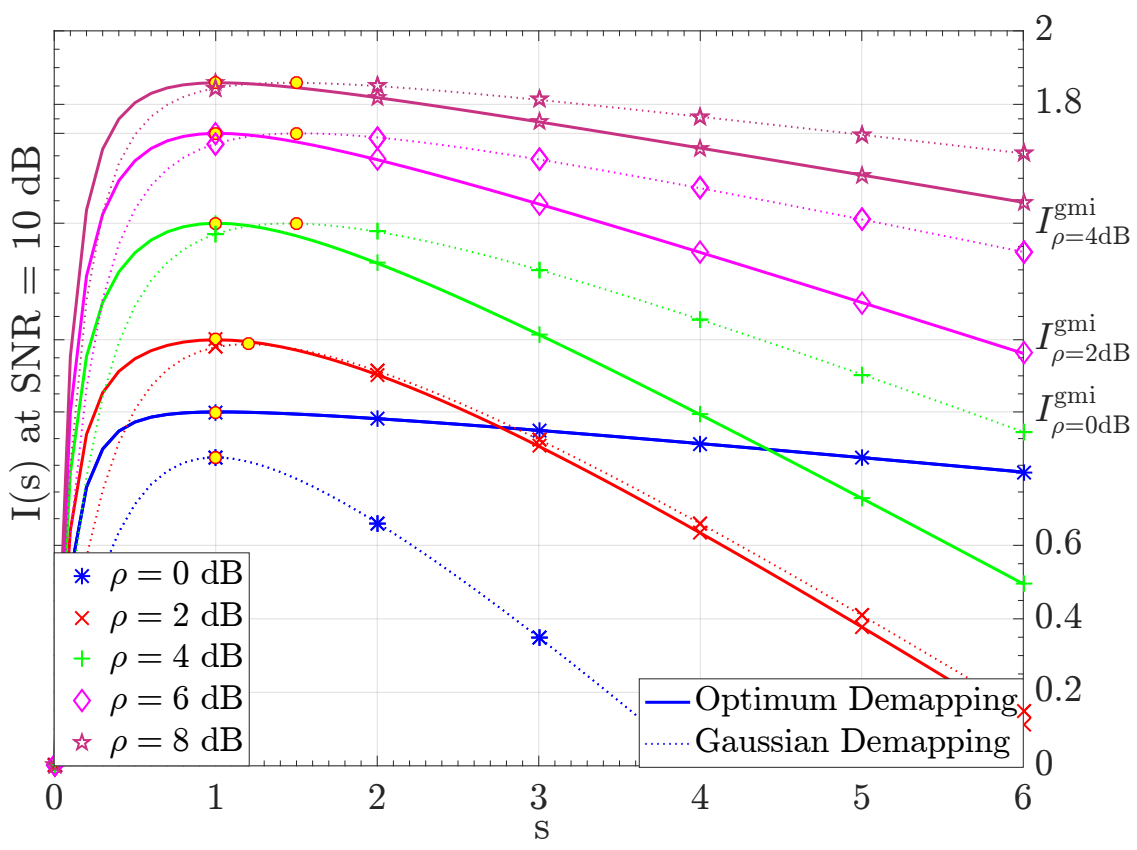

Figure 2.5: Top-layer signal $\left(x_{t}\right)$ QPSK + QPSK I-curves for different $s$ values at SNR $=10 \mathrm{~dB} \cdot \rho=0,2,4,6$, and $8 \mathrm{~dB}$.

Figure 2.4 depicts the achievable top-layer signal I-curves for a multi-layer signal constituted by a QPSK + QPSK constellation. GD and OD approaches are considered by using $\Lambda_{t}^{G D}\left(b_{i}\right)$ from $(2.2)$, and $\Lambda_{t}^{O D}\left(b_{i}\right)$ from $(2.7)$, respectively. Injection levels $\rho=0,2,4,6,8$, and $10 \mathrm{~dB}$ are evaluated in order to assess their influence in performance. The I-curves are calculated for a range of SNR ${ }^{1}$ values. Note that a matched demapper obtains the GMI at $s=1$ [78]. The results from the figure reveal that both the GD and OD alternatives perform very similar for the SNR region below $10 \mathrm{~dB}$. A clear improvement of the OD is found at higher SNR values. Moreover, for particular $\rho$ values, significant gains can be obtained with OD. Overall, the GD demapper seems to perform very well in noise-limited situations whereas the OD provides an advantage when the interference from the bottom-layer signal dominates. The results obtained via I-curves also reveal that system performance will be limited for certain code rates when using the GD. As an example, for $\rho=2 \mathrm{~dB}$, whereas

\footnotetext{
${ }^{1}$ Under the assumption of normalized transmission symbols $\mathrm{E}\left[|x|^{2}\right]=1$, the SNR is equivalent to the inverse of the noise variance $\mathrm{SNR}=1 / \sigma_{w}^{2}$
} 
the OD approach can provide $1.5 \mathrm{bps} / \mathrm{Hz}$ with a degradation of about $10 \mathrm{~dB}$ with respect to the single-layer case $(\rho=10 \mathrm{~dB})$, the GD approach cannot reach error-free reception.

On the other hand, I-curves have also been obtained for a QPSK + 64QAM signal. It was observed that at high SNR regions, the I-curves for the top-layer signal are reduced compared to QPSK + QPSK signal. This behavior refuses the conclusion extracted from [71]. The obtained results are not provided in this section, but performance results are presented and discussed in Section 2.4.

\subsubsection{Generalized Mutual Information Analysis}

In [76], the GMI is defined as the supremum of the I-curves relative to $s>0$

$$
I^{\mathrm{gmi}}=\sup _{s>0} I(s)
$$

As it was proved in [78], I-curves with $s=1$ provide $I^{\text {gmi }}$ when a matched pdf is considered. Therefore, the I-curves presented in Figure 2.4 only represents $I^{\text {gmi }}$ for OD, and an optimization of parameter $s$ may be applied to the GD. Figure 2.5 illustrates the I-curves with the same configuration and injection levels for $\mathrm{SNR}=10 \mathrm{~dB}$ and different $s$ values. As it can be observed, $I^{\mathrm{gmi}}$ is always obtained at $s=1$ for OD, but varies for GD between $s=\{1-$ $1.5\}$. This optimization process of $s$ is not straightforward on real receiver implementations. Reference [76] explains that an optimal LLR scaling in a mismatched decoder (as GD) can increase its performance in the same way as the optimization of $s$. Nevertheless, the implementation of a proper LLR metric scaling is out of the scope of the paper, as it would require of a more sophisticated receiver.

\subsection{Performance Analysis}

Next, the GD and OD approaches are evaluated by considering LDM in an ATSC 3.0 physical layer simulations ${ }^{2}$.

A comparison for the top-layer signal is conducted in Section 2.4.1 and 2.4.2, and for the bottom-layer signal in Section 2.4.3. Different MODCODs for the two signals as well as different injection levels $\rho$ have been assumed. An AWGN and an i.i.d. Rayleigh channel model for the top-layer signal are considered.

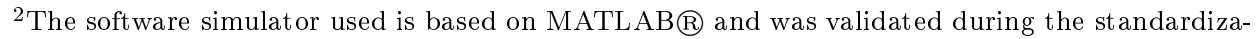
tion process of ATSC 3.0.
} 


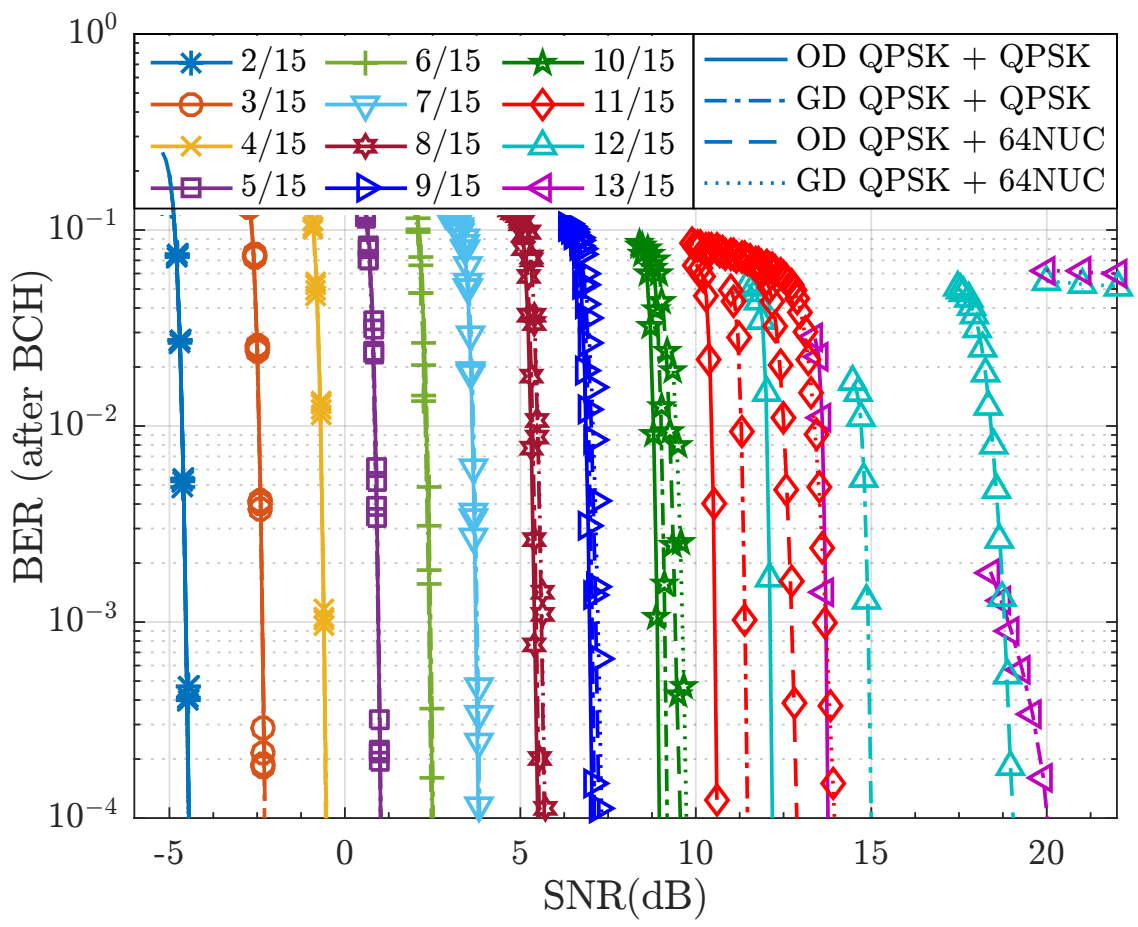

Figure 2.6: $x_{t}$ BER performance with $\rho=4$ dB. $x_{b}$ QPSK $13 / 15$ and 64NUC 13/15, AWGN channel

For the bottom-layer signal, an AWGN and a Rice (DVB-F1) channel model are considered. Ideal channel estimation is assumed. Table 2.1 presents a summary for the different considered ATSC 3.0 configurations[55].

\subsubsection{Performance of top-layer signal $\left(x_{t}\right)$ in AWGN channel}

Figure 2.6 shows the performance of the top-layer signal $x_{t}$ for the different MODCODs under study, when $x_{b}$ is set to either QPSK or 64NUC with $\rho=$ $4 \mathrm{~dB}$. It is shown that for $x_{t}$ coding rates below $7 / 15$, the SNR threshold of the four configurations are the same. Particularly, it can be observed that the SNR thresholds of $4 / 15$ and $6 / 15$ are, -0.5 , and $2.7 \mathrm{~dB}$, respectively, which fits with the results from [74]. The performance gains of OD are noticeable for SNRs greater than $10 \mathrm{~dB}$, i.e. from $10 / 15 x_{t}$ code rates. This confirms the conclusions in Section 2.3.2. Last, it can also be seen that if $x_{b}$ uses a $64 \mathrm{NUC}$ instead of QPSK, there is a performance degradation for $x_{t}$. Moreover, it can 
Table 2.1: Simulation Setup

\begin{tabular}{cccc}
\hline \hline \multicolumn{2}{c}{$\begin{array}{r}\text { Top-layer Performance } \\
\text { Configuration }\end{array}$} & $\begin{array}{r}\text { Bottom-Layer Performance } \\
\text { Configuration }\end{array}$ \\
\hline Parameter & Value & Parameter & Value \\
\hline$x_{t}$ MOD & 2 & $x_{t}$ MOD & 2 \\
\hline$x_{t}$ COD & $2 / 15-13 / 15$ & $x_{t}$ COD & $4 / 15,10 / 15$ \\
\hline$x_{b}$ MOD & $2,4,6,8$ & $x_{b}$ MOD & $2,4,6,8$ \\
\hline$x_{b} \mathrm{COD}$ & $13 / 15$ & $x_{b}$ COD & $4 / 15,10 / 15$ \\
\hline$\rho(\mathrm{dB})$ & $\{0-6\}$ & $\rho(\mathrm{dB})$ & 2,4 \\
\hline \multirow{3}{*}{ Channel } & $\begin{array}{c}\text { AWGN } \\
\text { i.i.d. Rayleigh }\end{array}$ & Channel & $\begin{array}{c}\text { AWGN } \\
\text { Rice (DVB-F1) }\end{array}$ \\
\hline
\end{tabular}

be seen that $x_{t} 12 / 15$ and $13 / 15$ cannot achieve error-free reception with the GD demapper if $x_{b}$ employs a $64 \mathrm{NUC}$ constellation.

For a more exhaustive performance study, the SNR thresholds of all $x_{t}, x_{b}$ and $\rho$ values established in Table 2.1 are shown in Figure 2.7 for AWGN channel. In top-left part of the figure, where both signals use a very robust QPSK modulation order (as in WiB systems), it can be observed that both demappers provide similar performance for low $\rho$ values at low $x_{t}$ coding rates $(3 / 15-$ $7 / 15)$. However, when the operational SNR is above $5 \mathrm{~dB}$, i.e. when $\rho$ is $1 \mathrm{~dB}$ or smaller, OD outperforming arises. Appreciable gains can also be observed at high $x_{t}$ coding rates $(8 / 15-13 / 15)$ from $\rho=5 \mathrm{~dB}$. These statements are applicable to the top-right part of the figure as well, where $x_{b}$ is using a $16 \mathrm{NUC}$ modulation order. In particular, for QPSK $13 / 15$ and $\rho=5 \mathrm{~dB}$, the SNR can be $3 \mathrm{~dB}$ lower with OD if a QPSK or a $16 \mathrm{NUC}$ is assumed for the $x_{b}$. This could also lead to a potential capacity increase for the same $\rho$ and SNR threshold. From the left part, assuming a fixed $\rho=3 \mathrm{~dB}$, whereas maximum $x_{t}$ MODCOD with GD for a $\mathrm{SNR}=13 \mathrm{~dB}$ is QPSK $10 / 15$, OD can increase the capacity allowing the use of QPSK $11 / 15$.

The bottom part of Figure 2.7 modifies the lower-layer signal constellation to a $64 \mathrm{NUC}$ or $256 \mathrm{NUC}$ (commonly used in ATSC 3.0 LDM studies). In these cases, compared to top part, it can be seen an $x_{t}$ performance degradation in high SNR regions for both demapping algorithms. Taking previous configuration, for QPSK $13 / 15$ and $\rho=5 \mathrm{~dB}$, the $x_{t}$ SNR can be $9 \mathrm{~dB}$ lower with OD if a $64 \mathrm{NUC}$ is assumed for the $x_{b}$. Furthermore, if a $256 \mathrm{NUC}$ is assumed for 

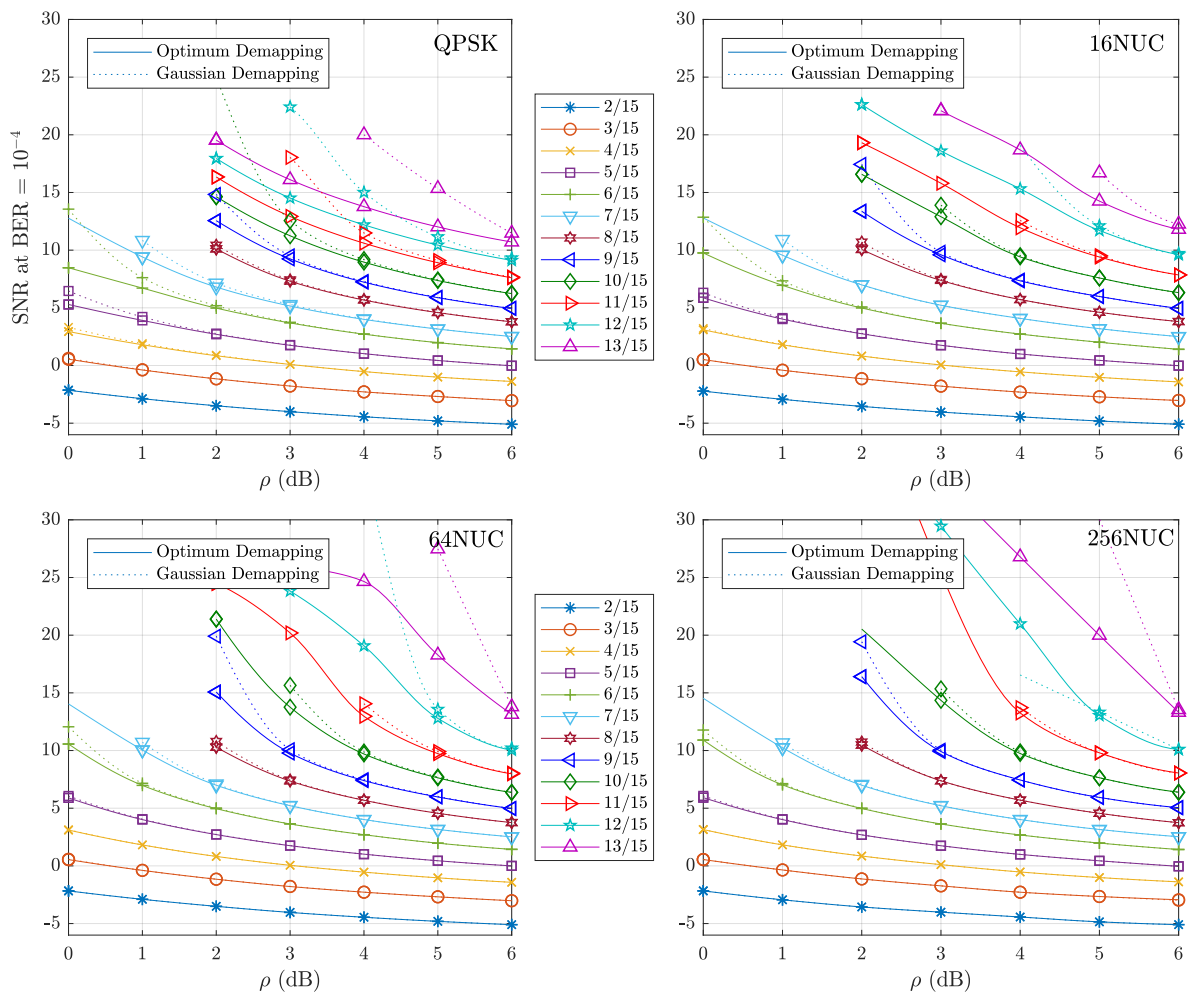

Figure 2.7: SNR thresholds for all the $x_{t}$ MODCODs and $\rho=1-6 \mathrm{~dB}$. $x_{b}$ QPSK (topleft), 16NUC (top-right), 64NUC (bottom-left), and 256NUC (bottom-right) in an AWGN channel.

the $x_{b}$ with $\rho=5 \mathrm{~dB}$, Quasi-Error Free (QEF) conditions cannot be achieved for GD. This demonstrates that the top-layer signal performance depends on the lower-layer signal constellation when the power ratios and $x_{t}$ coding rates are in the critical region. However, they are usually operational points to be avoided in service deployments. In [71], the impact of the $x_{b}$ constellation onto $x_{t}$ performance was only observed at practical regions for LDM operation, so that the system was only AWGN limited. The OD gains with respect to GD when either a QPSK or a $64 \mathrm{NUC}$ is used for the $x_{b}$ are summarized in Table 2.2. 
Table 2.2: OD gains (dB) for QPSK + QPSK and QPSK + 64NUC in AWGN channel

\begin{tabular}{cccccccc}
\hline \hline \multirow{2}{*}{$x_{t}$} & \multicolumn{7}{c}{$\rho(d B)$} \\
\cline { 2 - 8 } & 0 & 1 & 2 & 3 & 4 & 5 & 6 \\
\hline $2 / 15$ & $0.0 / 0.0$ & $0.0 / 0.0$ & $0.0 / 0.0$ & $0.0 / 0.0$ & $0.0 / 0.0$ & $0.0 / 0.0$ & $0.0 / 0.0$ \\
\hline $3 / 15$ & $0.0 / 0.0$ & $0.0 / 0.0$ & $0.0 / 0.0$ & $0.0 / 0.0$ & $0.0 / 0.0$ & $0.0 / 0.0$ & $0.0 / 0.0$ \\
\hline $4 / 15$ & $0.4 / 0.0$ & $0.1 / 0.0$ & $0.0 / 0.0$ & $0.0 / 0.0$ & $0.0 / 0.0$ & $0.0 / 0.0$ & $0.0 / 0.0$ \\
\hline $5 / 15$ & $1.2 / 0.2$ & $0.3 / 0.0$ & $0.0 / 0.0$ & $0.0 / 0.0$ & $0.0 / 0.0$ & $0.0 / 0.0$ & $0.0 / 0.0$ \\
\hline $6 / 15$ & $5.1 / 1.5$ & $0.9 / 0.2$ & $0.2 / 0.0$ & $0.0 / 0.0$ & $0.0 / 0.0$ & $0.0 / 0.0$ & $0.0 / 0.0$ \\
\hline $7 / 15$ & $-/-$ & $1.4 / 0.7$ & $0.3 / 0.1$ & $0.1 / 0.0$ & $0.0 / 0.0$ & $0.0 / 0.0$ & $0.0 / 0.0$ \\
\hline $8 / 15$ & $-/-$ & $-/-$ & $0.3 / 0.5$ & $0.1 / 0.0$ & $0.0 / 0.0$ & $0.0 / 0.0$ & $0.0 / 0.0$ \\
\hline $9 / 15$ & $-/-$ & $-/-$ & $2.3 / 4.8$ & $0.3 / 0.3$ & $0.1 / 0.0$ & $0.0 / 0.0$ & $0.0 / 0.0$ \\
\hline $10 / 15$ & $-/-$ & $-/-$ & $10.4 /-$ & $1.3 / 1.9$ & $0.2 / 0.2$ & $0.0 / 0.0$ & $0.0 / 0.0$ \\
\hline $11 / 15$ & $-/-$ & $-/-$ & $7.4 /-$ & $5.1 /-$ & $0.9 / 1.1$ & $0.2 / 0.2$ & $0.0 / 0.0$ \\
\hline $12 / 15$ & $-/-$ & $-/-$ & $8.1 /-$ & $7.9 /-$ & $2.8 / 15.9$ & $0.7 / 0.8$ & $0.2 / 0.2$ \\
\hline $13 / 15$ & $-/-$ & $-/-$ & $-/-$ & $-/-$ & $6.2 /-$ & $3.3 / 9.2$ & $0.8 / 0.7$ \\
\hline \hline
\end{tabular}

\subsubsection{Performance of top-layer signal $\left(x_{t}\right)$ in i.i.d. Rayleigh channel}

In order to assess the same study in a more realistic scenario, an i.i.d. Rayleigh fading channel modeling portable reception is assumed. Figure 2.8 presents the SNR thresholds for this channel and Table 2.3 summarizes the OD gains when $x_{b}$ uses a QPSK or a $64 \mathrm{NUC}$.

A general performance degradation can be observed in all configurations because of the more challenging conditions of this channel. From top figures, it can be seen that low $x_{t}$ coding rates $(3 / 15-7 / 15)$ perform similarly for both demapping algorithms at low $\rho$ values, as it occurred with AWGN channel. However, due to the performance degradation increase in portable reception conditions, performance differences can now be seen at a lower operational SNR. In particular, for the operational SNR of WiB systems $(0 \mathrm{~dB}$ for the proposed QPSK 1/2 MODCOD in [70]), $0.9 \mathrm{~dB}$ gains are obtained by OD for QPSK $2 / 15$ with $\rho=0 \mathrm{~dB}, 0.4 \mathrm{~dB}$ for QPSK $3 / 15$ with $\rho=2 \mathrm{~dB}$, and $0.1 \mathrm{~dB}$ for QPSK $4 / 15$ with $\rho=5 \mathrm{~dB}$. On the other hand, for high $x_{t}$ coding rates $(8 / 15-11 / 15)$, which are out of the WiB discussion, the same trend is followed, 
and noticeable OD gains are now observed from $\rho=6 \mathrm{~dB}$. Particularly, QPSK $12 / 15$ and $13 / 15$ with $\rho=5 \mathrm{~dB}$ can only achieve QEF with OD.

In another vein, the top-layer signal performance dependance on the lowerlayer signal constellation is confirmed for a portable reception scenario when top figures are compared with bottom ones. Furthermore, if $x_{b}$ is constituted by a $64 \mathrm{NUC}$ or a $256 \mathrm{NUC}, x_{t}$ performance degradation is observed for both demapping algorithms at high SNR regions, but also for GD at low SNR regions.
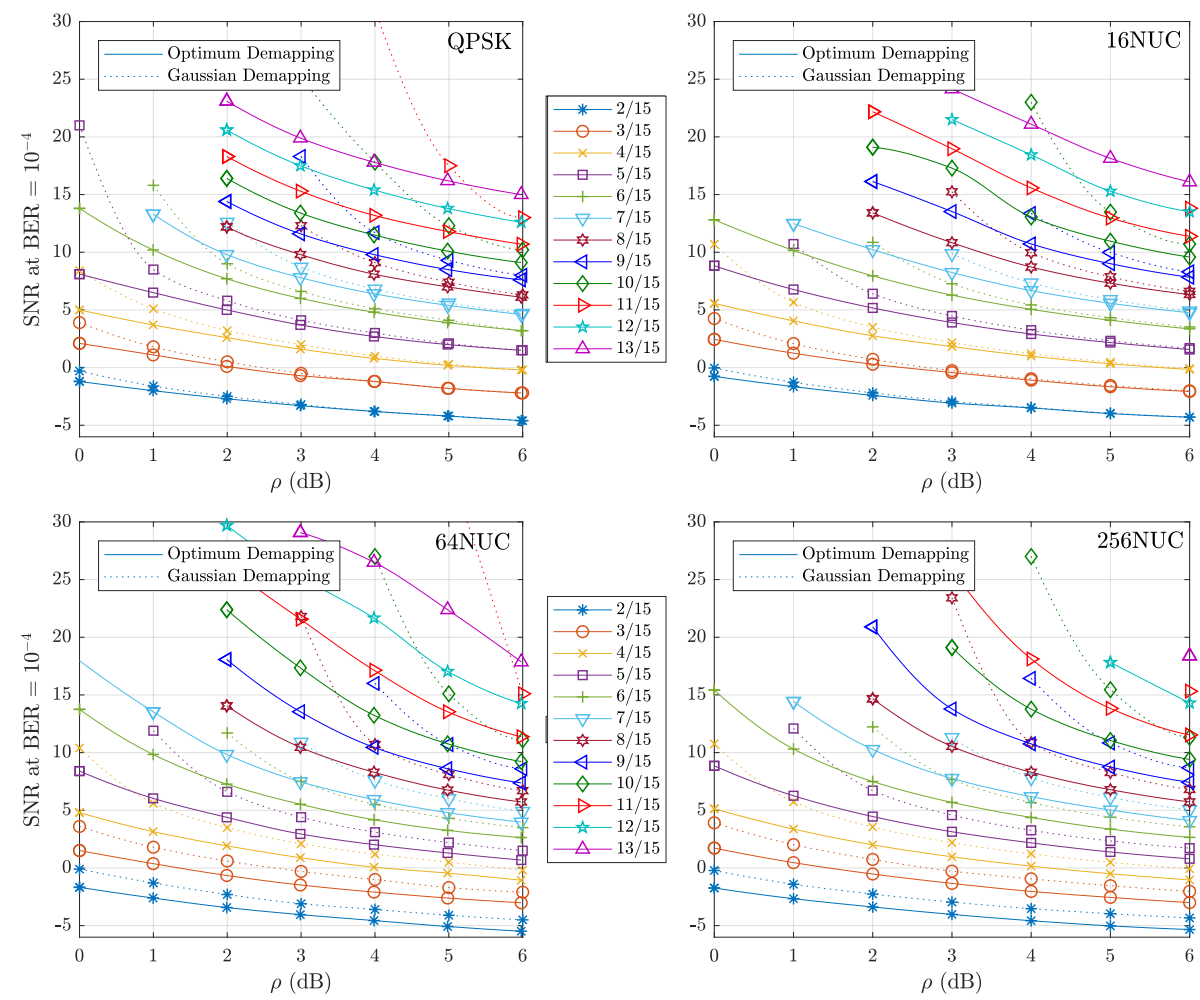

Figure 2.8: SNR thresholds for all the $x_{t}$ MODCODs and $\rho=1-6 \mathrm{~dB} . x_{b}$ QPSK (top-left), $16 \mathrm{NUC}$ (top-right), 64NUC (bottom-left), and 256NUC (bottom-right) in an i.i.d. Rayleigh channel. 
Table 2.3: OD gains $(\mathrm{dB})$ for QPSK + QPSK and QPSK + 64NUC in i.i.d. Rayleigh channel

\begin{tabular}{cccccccc}
\hline \hline \multirow{2}{*}{$x_{t}$} & \multicolumn{7}{c}{$\rho(d B)$} \\
\cline { 2 - 8 } & 0 & 1 & 2 & 3 & 4 & 5 & 6 \\
\hline $2 / 15$ & $0.9 / 0.9$ & $0.4 / 0.5$ & $0.2 / 0.3$ & $0.0 / 0.0$ & $0.1 / 0.1$ & $0.0 / 0.0$ & $0.0 / 0.0$ \\
\hline $3 / 15$ & $1.8 / 1.3$ & $0.7 / 0.6$ & $0.4 / 0.4$ & $0.2 / 0.3$ & $0.0 / 0.2$ & $0.0 / 0.0$ & $0.0 / 0.0$ \\
\hline $4 / 15$ & $3.4 / 4.9$ & $1.4 / 1.7$ & $0.6 / 0.8$ & $0.4 / 0.4$ & $0.2 / 0.3$ & $0.1 / 0.1$ & $0.0 / 0.0$ \\
\hline $5 / 15$ & $12.9 /-$ & $2 / 5.2$ & $0.8 / 1.5$ & $0.4 / 0.7$ & $0.3 / 0.3$ & $0.1 / 0.1$ & $0.0 / 0.0$ \\
\hline $6 / 15$ & $-/-$ & $5.6 /-$ & $1.3 / 3.8$ & $0.6 / 1.3$ & $0.3 / 0.6$ & $0.2 / 0.3$ & $0.0 / 0.1$ \\
\hline $7 / 15$ & $-/-$ & $-/-$ & $2.8 /-$ & $0.9 / 2.8$ & $0.4 / 1.0$ & $0.2 / 0.5$ & $0.1 / 0.2$ \\
\hline $8 / 15$ & $-/-$ & $-/-$ & $-/-$ & $2.5 / 10.8$ & $1.0 / 1.8$ & $0.4 / 0.7$ & $0.2 / 0.3$ \\
\hline $9 / 15$ & $-/-$ & $-/-$ & $-/-$ & $6.7 /-$ & $1.9 / 5.0$ & $0.8 / 1.5$ & $0.4 / 0.6$ \\
\hline $10 / 15$ & $-/-$ & $-/-$ & $-/-$ & $-/-$ & $6.3 /-$ & $2.2 / 3.8$ & $1.1 / 1.3$ \\
\hline $11 / 15$ & $-/-$ & $-/-$ & $-/-$ & $-/-$ & $-/-$ & $5.7 /-$ & $2.3 / 3.2$ \\
\hline \hline
\end{tabular}

\subsubsection{Performance of bottom-layer signal $x_{b}$}

The $x_{b}$ performance taking into account the soft-cancellation by the a-priori $x_{t}$ LLRs (SC) and the traditional $x_{t} \mathrm{HC}$ is evaluated next. To do so, different configurations have been considered: GD for the top-layer plus HC, and OD for the top-layer with both $\mathrm{HC}$ and SC for the bottom layer. Again, QPSK + QPSK, QPSK + 16NUC, QPSK + 64NUC, and QPSK + 256NUC configurations have been studied. Only $\rho=2$ and $4 \mathrm{~dB}$ are studied, as they represent low and high $\rho$ values, respectively. The $x_{b}$ SNRs at Bit Error Rate (BER) $=10^{-4}$ for AWGN channel and DVB-F1 channel are shown in Figure 2.9, and summarized in Table 2.4, and Table 2.5, respectively. DVB-F1 models a fixed reception channel, which is the potential target of the $x_{b}$ service.

For the WiB study case (QPSK + QPSK) and for QPSK +16NUC configuration, it can be observed that the use of $\mathrm{HC}$ or $\mathrm{SC}$ for the $x_{b}$ signal does not introduce significant gains for a robust $x_{t}$ MODCOD (4/15). The $x_{b}$ SNR threshold is mainly affected by the power reduction due to the injection level $\rho$. Nevertheless, when OD approach provides gains for the $x_{t}$, i.e. at high $x_{t}$ code rate $(10 / 15)$, the performance of the $x_{b}$ is also improved. This is particularly relevant in the case of $\rho=2 \mathrm{~dB}$ where GD cannot achieve QEF reception (grey bar) for $x_{t}$, and so for $x_{b}$. Regarding SC-HC comparison, it can be observed 
Table 2.4: OD and SC gains (dB) for AWGN channel

\begin{tabular}{cccccccccc}
\hline \hline \multirow{2}{*}{$x_{t}, x_{b}$} & \multirow{2}{*}{$\rho(\mathrm{dB})$} & \multicolumn{2}{c}{$Q P S K$} & \multicolumn{1}{c}{$16 N U C$} & \multicolumn{2}{c}{$64 N U C$} & \multicolumn{2}{c}{$256 N U C$} \\
\cline { 2 - 11 } & & $O D$ & $S C$ & $O D$ & $S C$ & $O D$ & $S C$ & $O D$ & $S C$ \\
\hline $4 / 15$ & 2 & 0.2 & 0.0 & 0.0 & 0.0 & 0.0 & 0.0 & 0.0 & 0.0 \\
\cline { 2 - 11 } $4 / 15$ & 4 & 0.0 & 0.0 & 0.0 & 0.0 & 0.0 & 0.0 & 0.0 & 0.0 \\
\hline $4 / 15$ & 2 & 0.0 & 0.0 & 0.0 & 0.0 & 0.0 & 0.0 & 0.0 & 0.0 \\
\cline { 2 - 11 } $10 / 15$ & 4 & 0.0 & 0.0 & 0.0 & 0.0 & 0.0 & 0.0 & 0.0 & 0.0 \\
\hline $10 / 15$ & 2 & - & - & - & - & $\infty$ & 3.9 & $\infty$ & 5.3 \\
$4 / 15$ & 4 & - & - & - & - & 0.2 & 0.1 & 0.0 & 0.0 \\
\hline $10 / 15$ & 2 & $\infty$ & 0.1 & $\infty$ & 0.1 & $\infty$ & 0.4 & $\infty$ & 0.2 \\
\cline { 2 - 10 } $10 / 15$ & 4 & 0.2 & 0.1 & 0.0 & 0.0 & 0.2 & 0.0 & 0.0 & 0.0 \\
\hline \hline
\end{tabular}

Table 2.5: OD and SC gains (dB) for DVB-F1 Rice channel

\begin{tabular}{cccccccccc}
\hline \hline \multirow{2}{*}{$x_{t}, x_{b}$} & \multirow{3}{*}{$\rho(\mathrm{dB})$} & \multicolumn{2}{c}{$Q P S K$} & \multicolumn{2}{c}{$16 N U C$} & \multicolumn{2}{c}{$64 N U C$} & \multicolumn{2}{c}{$256 N U C$} \\
\cline { 2 - 11 } & & $O D$ & $S C$ & $O D$ & $S C$ & $O D$ & $S C$ & $O D$ & $S C$ \\
\hline $4 / 15$ & 2 & 0.2 & 0.0 & 0.0 & 0.0 & 0.0 & 0.0 & 0.0 & 0.0 \\
\cline { 2 - 11 } $4 / 15$ & 4 & 0.0 & 0.0 & 0.0 & 0.0 & 0.0 & 0.0 & 0.0 & 0.0 \\
\hline $4 / 15$ & 2 & 0.0 & 0.0 & 0.0 & 0.0 & 0.0 & 0.0 & 0.0 & 0.0 \\
\cline { 2 - 11 } $10 / 15$ & 4 & 0.0 & 0.0 & 0.0 & 0.0 & 0.0 & 0.0 & 0.0 & 0.0 \\
\hline $10 / 15$ & 2 & - & - & - & - & $\infty$ & 3.8 & $\infty$ & 5.1 \\
\cline { 2 - 10 } $4 / 15$ & 4 & - & - & - & - & 1.0 & 0.2 & 0.0 & 0.0 \\
\hline $10 / 15$ & 2 & $\infty$ & 0.1 & $\infty$ & 0.1 & $\infty$ & 0.3 & $\infty$ & 0.2 \\
\cline { 2 - 9 } $10 / 15$ & 4 & 0.7 & 0.1 & 0.0 & 0.0 & 0.2 & 0.0 & 0.0 & 0.0 \\
\hline \hline
\end{tabular}



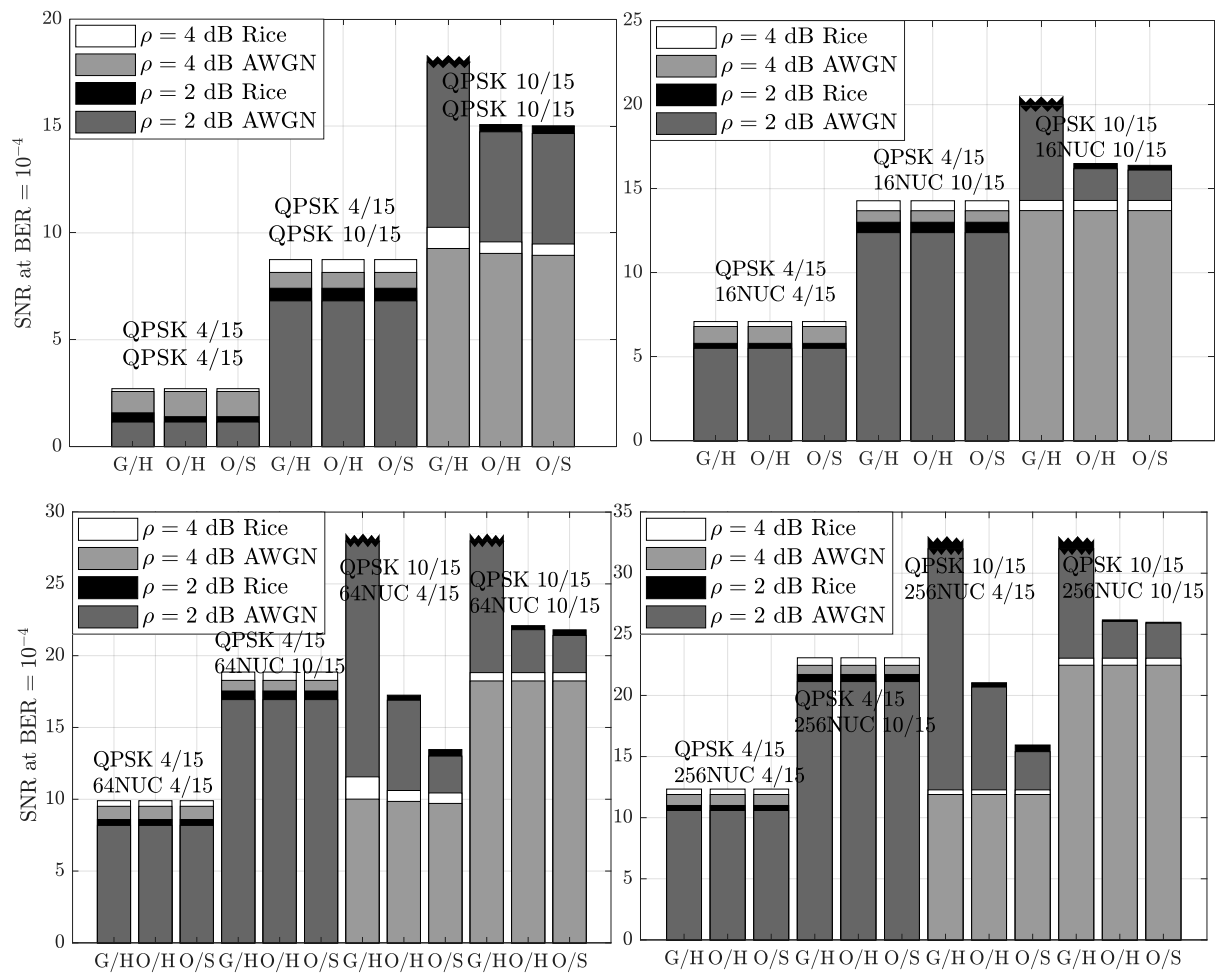

Figure 2.9: $x_{b}$ SNR thresholds for QPSK + QPSK (top-left), QPSK + 16NUC (topright), QPSK +64 NUC (bottom-left), and QPSK $+256 \mathrm{NUC}$ (bottom-right) with $\rho=2$ and $4 \mathrm{~dB}$ for AWGN and Rice channels (G/H: Gaussian Demapping and Hard-Cancellation, O/H: Optimum Demapping and Hard-Cancellation, O/S: Optimum Demapping and SoftCancellation).

that SC provides a slight $x_{b}$ improved performance in these conditions $(0,1 \mathrm{~dB})$ for both channels.

For the QPSK $+64 \mathrm{NUC}$, and QPSK $+256 \mathrm{NUC}$ cases, the differences in $x_{b}$ performance because of the top-layer signal demapping approach (GD vs OD) can be again noticed for the weak $x_{t}$ code rate $10 / 15$, when $\rho=2 \mathrm{~dB}$. On the other hand, the $x_{b}$ performance increase due to the use of SC is now increased. Large gains (about $4 \mathrm{~dB}$ for $64 \mathrm{NUC}$ and $5,3 \mathrm{~dB}$ for $256 \mathrm{NUC}$ ) can be achieved if a robust $x_{b}$ code rate is configured, but also are observed with high $x_{b}$ code rate (around $0,3 \mathrm{~dB}$ for both $x_{b}$ modulation orders) for AWGN channel. 
Similar gains are obtained when the more realistic fixed-rooftop channel is assumed. From Table 2.5, $3.8 \mathrm{~dB}$ gains are obtained by SC when $x_{b}$ is constituted by a $64 \mathrm{NUC} 4 / 15$ and $5.1 \mathrm{~dB}$ when is formed by a $256 \mathrm{NUC} 4 / 15)$.

\subsection{Conclusions}

This paper studies different demapping approaches for multi-layer broadcast systems from a generic point of view. Underlying signals have commonly been assumed as AWGN-like interference when demapping the top-layer constellation (GD). As previously introduced in [71], this assumption may not be valid when the power of the layers is similar and high code-rates are configured for the top-layer signal.

The paper provides results in terms of error exponent and generalized mutual information by means of Monte-Carlo simulations, covering a wide range of operational points. The performance has been crosschecked with ATSC 3.0 physical layer simulations and compared to the results presented in [71]. The optimum demapping (OD) approach, which considers the knowledge of the symbol alphabet of the underlying constellation brings potential gains at the expense of a complexity increase comparable to a higher modulation order (in terms of Euclidean distances to be computed). It was observed that OD gains depend on the power ratio between layers $(\rho)$, the top-layer code-rate and the lower-layer constellation. They vary from $0 \mathrm{~dB}$ (at high $\rho$, and low top-layer signal code-rate) up to $10 \mathrm{~dB}$ (at low $\rho$, and high top-layer signal code rate). Moreover, the OD method brings a performance increase up to 4 $\mathrm{dB}$ for the underlying layers when a cancellation method based on soft a-priori information transfer (SC) is applied.

The expected gains by the OD demapping may be useful in systems employing robust signals (i.e. QPSK modulation order) with low power differences. Although the operation points in which gains are obtained are less attractive for ATSC 3.0 LDM operation, systems such as WiB can benefit from high gains when e.g. the same QPSK signal is transmitted from multiple stations.

Further studies should consider the performance analysis using other fading channel models, as well as the impact of introducing more than two layers. In addition, since the a-priori information transfer from top to bottom layer signals have been demonstrated to significantly improve performance, an iterative extension, also considering the transfer from bottom to top layer, should be analyzed as well as their implications in terms of complexity. Other implemen- 
tation aspects, such as the increased power consumption by LDPC decoders in low SNR conditions, can also be considered. 

Chapter 3

\section{Scattered Pilot Performance and Optimization for ATSC 3.0}

E. Garro, J.J. Gimenez, S.I. Park, D. GomezBarquero, IEEE Trans. on Broadcast., vol. 63, no. 1, pp. 282-292, March 201\%. The next-generation U.S. Digital Terrestrial Television (DTT) standard ATSC 3.0 is the most flexible DTT standard ever developed, outperforming the state-of-theart DVB-T2 standard. This higher flexibility allows broadcasters to select the configuration that better suits the coverage and capacity requirements per service. Regarding the selection of pilot patterns, whereas DVB-T2 provides 8 different patterns with a unique pilot amplitude, ATSC 3.0 expands up to 16, with 5 different amplitudes per pattern. This paper focuses on the pilot pattern and amplitude performance and optimization for time and power multiplexing modes, TDM and LDM respectively, of ATSC 3.0. The selection of the optimum pilot configuration is not straightforward. On the one hand, the pilots must be sufficiently dense to follow channel fluctuations. On the other hand, as long as pilot density is increased, more overhead is introduced. Moreover, this selection is particularly essential in LDM mode, because the LDM implementation in ATSC 3.0 requires that both layers share all the waveform parameters, including pilot pattern configuration. In addition, there is an error proportional to the channel estimate of the top layer that affects to the lower layer performance. 


\subsection{Introduction}

The Advanced Television Systems Committee (ATSC) has released the next-generation U.S. Digital Terrestrial Television (DTT) standard, known as ATSC - Third Generation (ATSC 3.0) [26]. It outperforms current terrestrial broadcasting state-of-the-art standard, DVB - Terrestrial Second Generation (DVB-T2) [25] increasing transmission flexibility to meet broadcasters' requirements. It provides a higher spectral efficiency and extends into a wider operating range in terms of Signal-to-Noise Ratio (SNR) [26].

The selection of the transmission configuration that guarantees the maximum capacity (data-rate) for the desired coverage (robustness) is the main planning goal of a broadcaster. The direct approach is to select the appropriate Modulation and Coding Rate (MODCOD) that satisfies these requirements [55]. For such case, ATSC 3.0 provides a larger granularity in SNR, and improves the performance by using new Low Density Parity Check (LDPC) codes [79] and Non-Uniform Constellation (NUC) [28]. In particular, there are 2 LDPC code lengths (16200 and 64800 bits) in both DTT standards, but whereas DVB-T2 allows 6 code rates and 4 modulation orders, in ATSC 3.0 there are 12 code rates (from $2 / 15$ to $13 / 15$ ) and 6 modulation orders (from Quadrature Phase-Shift Keying (QPSK) to 4096Quadrature Amplitude Modulation (QAM)). Hence, while DVB-T2 offers a performance ranging from 1 to 22 dB SNR under Additive White Gaussian Noise (AWGN) channel conditions, in ATSC 3.0 it ranges from $-6.2 \mathrm{~dB}$ to $32 \mathrm{~dB}$.

Regarding multiplexing modes, whereas DVB-T2 offers Time Division Multiplexing (TDM) to carry services aimed at different reception conditions, ATSC 3.0 supports three options, time, frequency and power multiplexing modes, the last one known as Layered Division Multiplexing (LDM) [45]. In LDM, the transmitted signal consists of two independent signals, namely layers, superimposed together by assigning different power to each one, according to the Injection Level $(\Delta)$. Thus, whereas TDM mode reduces the capacity of the multiplexed services by not using $100 \%$ of time resources, LDM fully exploits the channel capacity by each multiplexed service, but in return it modifies the SNR threshold.

ATSC 3.0 has also increased the flexibility of the waveform generation parameters, i.e. Time Interleaver (TIL), Scattered Pilot (SP), Fast Fourier Transform (FFT) and Guard Interval (GI). TIL length has been increased by using a sheer convolutional TIL (CTI). In addition, ATSC 3.0 provides up to 16 different SP, with up to 5 different amplitudes for each one, known as pilot 


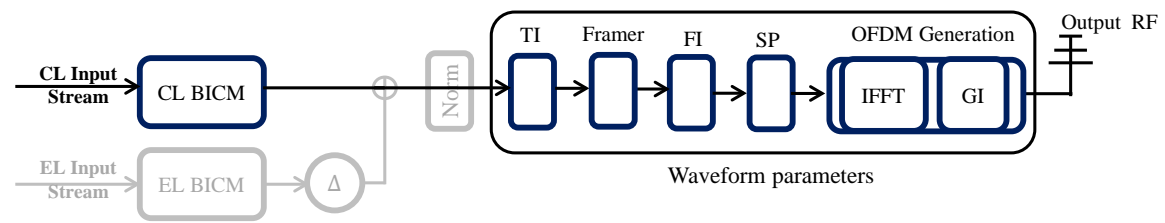

Figure 3.1: ATSC 3.0 transmitter block diagram. Grey blocks are only enabled when LDM mode is used. Each LDM layer passes through an independent BICM process. They are then aggregated, so that they share the same TIL length, SP, FFT size and GI length. At the receiver, only one channel estimation for both layers is performed.

boostings. It has also increased the FFT/GI combinations. There are 3 FFT sizes $(8 \mathrm{~K}, 16 \mathrm{~K}$ and $32 \mathrm{~K}$ ) and $12 \mathrm{GI}$ lengths (from 27 to $700 \mu \mathrm{s}$ ).

Different studies have shown the impact of the TIL, FFT, and GI in ATSC 3.0 [56], [59]. However, the impact of SP has only been partially evaluated in [80]. Hence, the proposed paper is focused on the pilot configuration optimization for TDM and LDM modes of ATSC 3.0. It could be assumed that the SP must be sufficiently dense to follow channel fluctuations. Nevertheless, at the same time, as long as pilot density is increased, more data overhead is introduced. In addition, the possibility of using 5 pilot boostings for each SP makes the selection even more tricky. In another vein, it should be noted that the LDM implementation in ATSC 3.0 requires that both layers share all the waveform parameters [51], including SP, in order to limit receiver's complexity. Thus, a trade-off between the optimum configuration for the mobile layer (higher robustness by a denser SP), and for the fixed layer (higher capacity by a sparser SP) arises. The selected configuration also affects channel estimation, and the estimate error affects the demodulation of the lower layer.

The paper is structured as follows: Section 3.2 overviews the ATSC 3.0 transmitter and receiver waveform parameters. The impact in performance due to the channel estimation at receiver is presented in Section 3.3. Section 3.4 describes the methodology and the simulation setup followed for performance evaluation. The results assessed by physical layer simulations are presented in Section 3.5. Finally, the conclusions are summarized in Section 3.6. 


\subsection{ATSC 3.0 Waveform Overview}

Figure 3.1 presents the ATSC 3.0 transmitter block diagram. The input stream passes through a BICM chain. Next, the waveform processing is performed. The selection of the configuration for every waveform parameter leads to different capacity - robustness trade-offs. A brief explanation of each one is presented next.

- Time Interleaver (TIL): increases the robustness of the system against impulsive noise and time selective fading thanks to the time diversity introduced. However, it increases the demodulation latency and limits the maximum data rate of the service [30], [81].

- Frequency Interleaver (FIL): Increases frequency diversity. It is performed throughout the complete channel bandwidth on a per Orthogonal Frequency-Division Multiplexing (OFDM) symbol basis to separate burst errors in the frequency domain [82].

- Scattered Pilot Pattern (SP): Pilots are carriers that do not contain net information but whose value is known by the receiver in order to get a proper channel estimation at pilot positions. Next, the channel estimates at data cells are obtained by interpolation. SP must be sufficiently dense to follow frequency channel fluctuations $\left(D_{x}\right)$ and time channel fluctuations $\left(D_{y}\right)$ [83]. More details are given in Section 3.3.

- Inverse Fast Fourier Transform (IFFT): OFDM systems, as ATSC 3.0, are very sensitive to Inter-Carrier Interference (ICI), which depends on the FFT size. The smaller the FFT size, the more ICI the system can withstand. ATSC 3.0 has adopted three different FFT sizes $(8,16$, and $32 \mathrm{k})$.

- Guard Interval (GI): ATSC 3.0 has adopted twelve GI lengths: 192, 384, 512, 768, 1024, 1536, 2048, 2432, 3072, 3648, 4096, and 4864 samples. GI length must be, at least, equal to the length of the multipath channel in order to limit Inter-Symbol Interference (ISI), which is important for Single Frequency Networks (SFN) topologies. As long as GI is increased, the longer the SFN distance allowed. However, it also increases the overhead. Thus, not all GI lengths are allowed for the three FFT sizes, as shown in Table III in [26].

Figure 3.1 also illustrates the processing when LDM mode is used, by the use of grey blocks in the figure. In such case, there are two input streams. The robust 
one, passes through a Core Layer (CL) BICM chain. The second input stream, providing a high data rate service and known as Enhanced Layer (EL), passes through a second and independent EL BICM chain. Both layers are then added by assigning a $\Delta \mathrm{dB}$ power reduction to the EL with respect to the CL. Last, waveform processing is performed. As it can be seen, the waveform processing is common for both LDM layers and, hence, channel estimation is performed only once at receivers. As each layer is intended for different reception conditions, the common waveform parameters restriction leads LDM to additional commitments regarding capacity - robustness trade-offs.

\subsection{Channel Estimation in ATSC 3.0}

Since the radio channel is frequency selective and time-varying, a dynamic estimation of the channel is needed. Channel estimation is performed by inserting scattered pilot subcarriers into the OFDM symbols. The pilot-based channel estimation consists of different algorithms to estimate the channel at the SP that varies among receivers. An interpolation of the channel across data cells is next needed. This interpolation could be frequency-only or a 2dimensional time/frequency interpolation, which depends on the SP pattern assumed. Therefore, there are two terms that affect the good or bad estimation of the Channel Frequency Response (CFR): scattered pilot configuration used by the broadcaster and the channel estimator employed by receivers.

\subsubsection{Scattered Pilot Configuration assumed at Transmitters}

The scattered pilot configuration is divided into two parameters: SP pattern, which defines the amount and location of the scattered pilots inside the ATSC 3.0 frames, and the pilot boosting, which defines their amplitude with respect to data carriers. There are some considerations for each parameter.

\section{Scattered Pilot Pattern}

As the CFR varies with both time and frequency, the SP is characterized by two terms, the frequency separation of pilots, $D_{x}$, and the length of the SP in OFDM symbols, $D_{y}$. The 16 different SP patterns of ATSC 3.0 are extracted from $8 D_{x}$ values $(3,4,6,8,12,16,24$, and 32$)$ and from $2 D_{y}$ values $(2$, 4). As an example, Figure 3.2 illustrates the pilot distribution over 8 OFDM symbols, assuming an SP with $D_{x}=3$ and $D_{y}=2$. Table 3.1 presents the SP with their corresponding overhead in ATSC 3.0. 


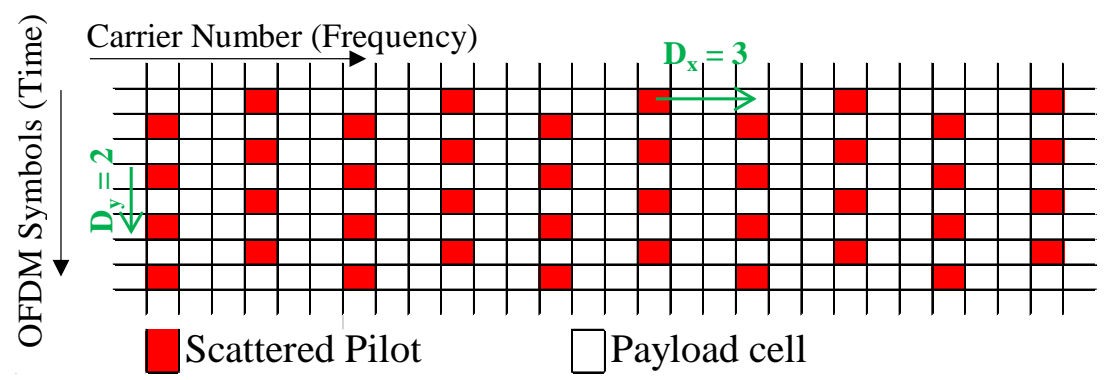

Figure 3.2: SP3_2 $\left(D_{x}=3, D_{y}=2\right)$.

Table 3.1: ATSC 3.0 SP Patterns

\begin{tabular}{cccccccc}
\hline \hline SP & $D_{x}$ & $D_{y}$ & Ov. (\%) & $S P$ & $D_{x}$ & $D_{y}$ & Ov. (\%) \\
\hline SP3_2 & 3 & 2 & 16.7 & $S P 8 \_4$ & 8 & 4 & 3.1 \\
\hline SP4_2 & 4 & 2 & 12.5 & SP16_2 & 16 & 2 & 3.1 \\
\hline SP3_4 & 3 & 4 & 8.3 & SP12_4 & 12 & 4 & 2.1 \\
\hline SP6_2 & 6 & 2 & 8.3 & SP24_2 & 24 & 2 & 2.1 \\
\hline SP4_4 & 4 & 4 & 6.3 & SP16_4 & 16 & 4 & 1.6 \\
\hline SP8_2 & 8 & 2 & 6.3 & SP32_2 & 32 & 2 & 1.6 \\
\hline SP6_4 & 6 & 4 & 4.2 & SP24_4 & 24 & 4 & 1.0 \\
\hline SP12_2 & 12 & 2 & 4.2 & SP32_4 & 32 & 4 & 0.8 \\
\hline \hline
\end{tabular}

At first, it could be assumed that the densest SP provides the most accurate channel estimation. Nevertheless, at the same time, as it can be seen in Table 3.1, it introduces the highest overhead. In addition, the values of $D_{x}$ and $D_{y}$ must be selected according to the CFR characteristics. The particular $D_{x}$ and $D_{y}$ implications are presented next.

Separation between pilot carriers $\left(D_{x}\right)$ The last path that can contribute constructively so that it can be correctly equalized by a receiver depends on the channel delay spread, i.e. the coherence bandwidth. According to the Nyquist sampling theorem, this limit when both time and frequency interpolation are implemented [83] is estimated as:

$$
T_{n}=\frac{T_{U}}{D_{x}}
$$


where $T_{n}$ represents the Nyquist limit and $T_{U}$ is the useful symbol duration.

For ATSC 3.0 it has been assumed that receivers are only able to correctly equalize those signals with echoes up to $75 \%$ or $89 \%$ of Nyquist limit. That is, only those GIs which length is shorter than $75 \%$ or $89 \%$ of $T_{n}$ are allowed. This ratio is also known as Guard Utilization Ratio (GUR). It can be seen that Nyquist limit is increased with the useful symbol duration, i.e. with the FFT size, and reduced with the SP. Hence, not all GIs are allowed for each SP and FFT size.

Length of pattern in symbols $\left(D_{y}\right)$ If the transmitted signal is expected to be received in mobility conditions, the channel will vary across OFDM symbols. Thus, the pilots need to be inserted at a certain rate $\left(D_{y}\right)$ that is a function of the coherence time, which is related to the Doppler shift limit. As symbols occur at the rate $f_{S}=1 /\left(T_{U}+T_{G}\right) \mathrm{Hz}$, the Doppler shift limit for frequency channel variation, $f_{D}$, that can be measured is:

$$
f_{D}=\frac{ \pm 1}{2 D_{y} \cdot\left(T_{U}+T_{G}\right)} H z
$$

where $T_{G}$ is the GI length in time.

From the expression it can be observed that the smaller the $D_{y}$, the GI, and the FFT size, the higher the Doppler shift limit. Hence, in order to support high speeds $D_{y}=4$ was discarded for $32 \mathrm{k}$ FFT size.

As summary, taking the Nyquist $\left(D_{x}\right)$ and Doppler $\left(D_{y}\right)$ limits into account, the different FFT/GI-SP combinations allowed in ATSC 3.0 are presented in Table 3.2.

\section{Pilot Boosting}

The other pilot parameter that affects the performance is the pilot boosting. In order to provide a reasonable trim, the equalized data $\operatorname{SNR}\left(S N R_{E q}\right)$ was considered as a good metric for obtaining the best overall performance taking into account different receiver equipment. It is estimated as:

$$
S N R_{E Q, b}=\frac{\sigma_{s}^{2} \times k}{\sigma_{N}^{2}+\sigma_{N}^{2} \times f_{i n t} / b}=S N R \times \frac{k}{1+f_{\text {int }} / b}
$$

where $\sigma_{s}^{2}$ is the data signal variance, $\sigma_{N}^{2}$ is the noise variance, $b$ is the SP boosting factor, $k$ is the power normalization $\left(k=D_{x} \cdot D_{y} /\left(D_{x} \cdot D_{y}-1\right)+\right.$ 
Table 3.2: SP pattern to be used for each allowed FFT and GI combination

\begin{tabular}{|c|c|c|c|}
\hline$G I$ & $8 k F F T$ & $16 k F F T$ & $32 k F F T$ \\
\hline GI1_192 & $\begin{array}{l}\text { SP } 32 \_2, \mathrm{SP} 32 \_4 \\
\text { SP16_2, SP16_4 }\end{array}$ & SP32_2, SP32_4 & SP32_2 \\
\hline GI2_384 & $\begin{array}{c}\mathrm{SP} 16 \_2, \mathrm{SP} 16 \_4 \\
\mathrm{SP} 8 \_2, \mathrm{SP} 8 \_4\end{array}$ & $\begin{array}{l}\text { SP } 32 \_2, \mathrm{SP} 32 \_4 \\
\text { SP16_2, SP16_4 }\end{array}$ & SP32_2 \\
\hline$G I 3 \_512$ & $\begin{array}{c}\mathrm{SP} 12 \_2, \mathrm{SP} 12 \_4 \\
\mathrm{SP} 6 \_2, \mathrm{SP} 6 \_4\end{array}$ & $\begin{array}{l}\mathrm{SP} 24 \_2, \mathrm{SP} 24 \_4 \\
\mathrm{SP} 12 \_2, \mathrm{SP} 12 \_4\end{array}$ & SP24_2 \\
\hline GI4_ 768 & $\begin{array}{l}\mathrm{SP} 8 \_2, \mathrm{SP} 8 \_4, \\
\mathrm{SP} 4 \_2, \mathrm{SP} 4 \_4\end{array}$ & $\begin{array}{c}\mathrm{SP} 16 \_2, \mathrm{SP} 16 \_4 \\
\mathrm{SP} 8 \_2, \mathrm{SP} 8 \_4\end{array}$ & SP $32 \_2, \mathrm{SP} 16 \_2$ \\
\hline GI5_1024 & $\begin{array}{l}\mathrm{SP} 6 \_2, \mathrm{SP} 6 \_4, \\
\mathrm{SP} 3 \_2, \mathrm{SP} 3 \_4\end{array}$ & $\begin{array}{l}\mathrm{SP} 12 \_2, \mathrm{SP} 12 \_4 \\
\mathrm{SP} 6 \_2, \mathrm{SP} 6 \_4\end{array}$ & SP24_2, SP12_2 \\
\hline GI6_1536 & $\mathrm{SP} 4 \_2, \mathrm{SP} 4 \_4$ & $\begin{array}{l}\mathrm{SP} 8 \_2, \mathrm{SP} 8 \_4 \\
\mathrm{SP} 4 \_2, \mathrm{SP} 4 \_4\end{array}$ & SP16_2, SP8_2 \\
\hline$G I^{\prime}=2048$ & $\mathrm{SP} 3 \_2, \mathrm{SP} 3 \_4$ & $\begin{array}{l}\text { SP6_2, SP6_4, } \\
\text { SP3_2, SP3_4 }\end{array}$ & SP12_2, SP6_2 \\
\hline GI8_2432 & - & $\begin{array}{l}\mathrm{SP} 6 \_2, \mathrm{SP} 6 \_4, \\
\mathrm{SP} 3 \_2, \mathrm{SP} 3 \_4\end{array}$ & SP12_2, SP6_2 \\
\hline GI9_3072 & - & $\mathrm{SP} 4 \_2, \mathrm{SP} 4 \_4$ & $\mathrm{SP} 8 \_2, \mathrm{SP} 4 \_2$ \\
\hline GI10_3648 & - & $\mathrm{SP} 4 \_2, \mathrm{SP} 4 \_4$ & $\mathrm{SP} 8 \_2, \mathrm{SP} 4 \_2$ \\
\hline GI11_4096 & - & SP3_2, SP3_4 & SP6 $62, \mathrm{SP} 3 \_2$ \\
\hline GI12_4864 & - & - & SP6_2, SP3_2 \\
\hline
\end{tabular}

b), and $f_{\text {int }}=f_{\text {int,time }} \times f_{\text {int,freq }}$ is the noise reduction factor by time and frequency interpolation. As $f_{\text {int }}$ varies depending on receiver manufacturers, the five different boosting values of ATSC 3.0 (from 0 to 4 ) are extracted from $f_{\text {int }, \text { freq }}=\{0,0.25,0.5,0.75,1\}$. Figure 3.3 presents the process of how to select the optimum boosting value for each SP with $f_{\text {int }, \text { freq }}=0.5$. The pilot boosting for the SP patterns of ATSC 3.0 are listed in Table 3.3.

On the other hand, although higher pilot boosting improves channel estimation accuracy, it also decreases the power of the data carriers, and so does the overall SNR of the system. This data cell power reduction is approximated as an SNR 


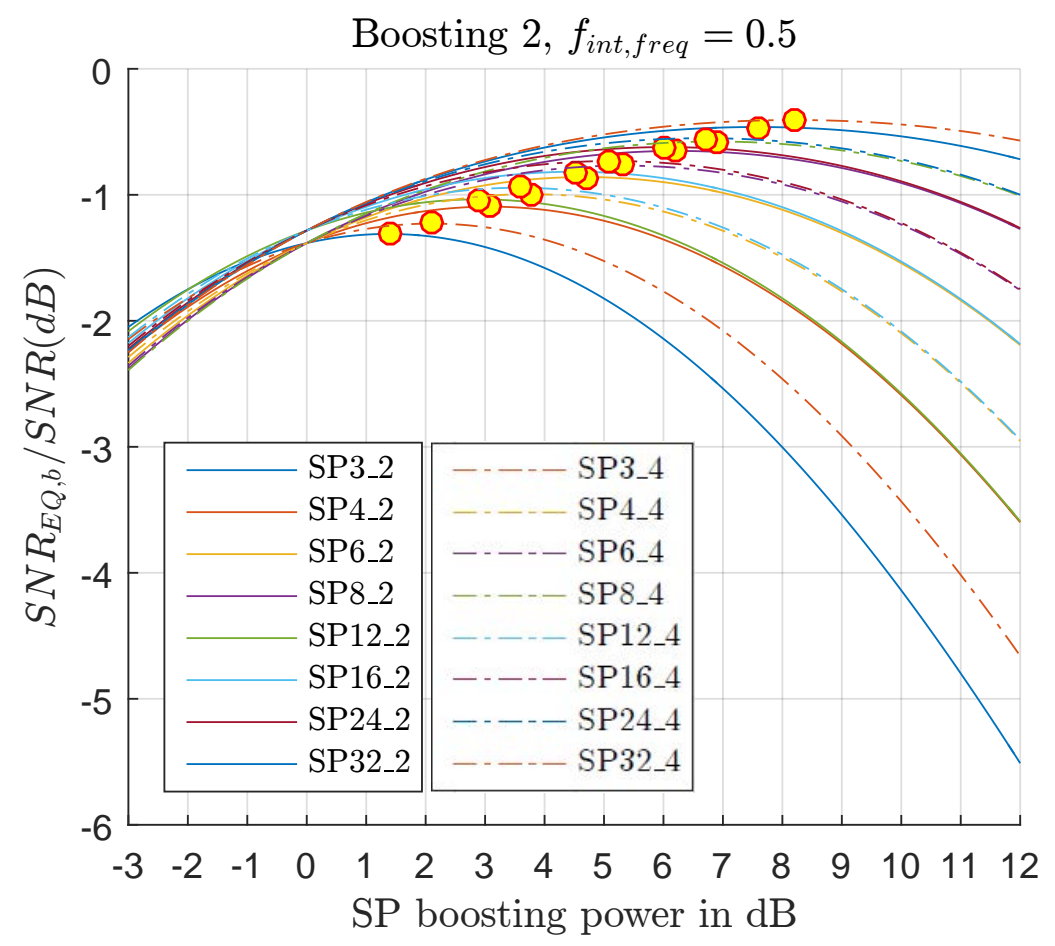

Figure 3.3: Equalized SNR performance and optimum boosting value for all SP patterns assuming a $f_{\text {int }, \text { freq }}=0,5$

reduction, and it can be estimated as:

$$
\Delta_{B P}(\mathrm{~dB})=10 \cdot \log _{10} \frac{N_{d a t a}+N_{S P} \cdot A_{S P}^{2}}{N_{\text {data }}+N_{S P}}
$$

where $N_{\text {data }}$ refers to the number of data cells per OFDM symbol, $N_{S P}$ refers to the number of scattered pilots per OFDM symbol, and $A_{S P}$ refers to pilot boosting relative to data cells. The corresponding data cell power reduction for each pilot boosting and SP of ATSC 3.0 is listed in Table $3.4^{1}$.

\footnotetext{
${ }^{1}$ Continual and edge pilots have not been considered in Equation (3.4) and Table 3.4
} 
Table 3.3: ATSC 3.0 Scattered Pilot Boosting $(d B)$

\begin{tabular}{cccccc}
\hline \hline \multirow{2}{*}{ SP } & \multicolumn{5}{c}{ Boosting b $(d B)$} \\
\cline { 2 - 6 } & 0 & 1 & 2 & 3 & 4 \\
\hline SP3_2 & 0 & 0 & 1.40 & 2.20 & 2.90 \\
\hline SP3_4 & 0 & 1.40 & 2.90 & 3.80 & 4.40 \\
\hline SP4_2 & 0 & 0.60 & 2.10 & 3.00 & 3.60 \\
\hline SP4_4 & 0 & 2.10 & 3.60 & 4.40 & 5.10 \\
\hline SP6_2 & 0 & 1.60 & 3.10 & 4.00 & 4.60 \\
\hline SP6_4 & 0 & 3.00 & 4.50 & 5.40 & 6.00 \\
\hline SP8_2 & 0 & 2.20 & 3.80 & 4.60 & 5.30 \\
\hline SP8_4 & 0 & 3.60 & 5.10 & 6.00 & 6.60 \\
\hline SP12_2 & 0 & 3.20 & 4.70 & 5.60 & 6.20 \\
\hline SP12_4 & 0 & 4.50 & 6.00 & 6.90 & 7.50 \\
\hline SP16_2 & 0 & 3.80 & 5.30 & 6.20 & 6.80 \\
\hline SP16_4 & 0 & 5.20 & 6.70 & 7.60 & 8.20 \\
\hline SP24_2 & 0 & 4.70 & 6.20 & 7.10 & 7.70 \\
\hline SP24_4 & 0 & 6.10 & 7.60 & 8.50 & 9.10 \\
\hline SP32_2 & 0 & 5.40 & 6.90 & 7.70 & 8.40 \\
\hline SP32_4 & 0 & 6.70 & 8.20 & 9.10 & 9.70 \\
\hline \hline
\end{tabular}

\subsubsection{Channel Estimator implemented at Receivers}

The second term that affects the good or bad estimation of the CFR is the estimator implemented at receivers. The use of one or another estimator by the receiver has a significant impact on the expected performance. There are different techniques to estimate the CFR.

Channel estimation in OFDM is a two dimensional (2-D) problem that varies with time and frequency. 2-D methods could be applied to estimate the channel from pilots. However, due to the computational complexity of 2-D estimators, it is commonly simplified by a cascade of two 1-D problems. In such case, complexity reductions can be achieved with reasonable performance loss [84]. 
Table 3.4: ATSC 3.0 Data Power Reduction $(d B)$

\begin{tabular}{cccccc}
\hline \hline \multirow{2}{*}{$S P$} & \multicolumn{5}{c}{$\Delta_{B P}(d B)$} \\
\cline { 2 - 6 } & 0 & 1 & 2 & 3 & 4 \\
\hline SP3_2 & 0 & 0 & 0.26 & 0.46 & 0.65 \\
\hline SP3_4 & 0 & 0.13 & 0.34 & 0.49 & 0.60 \\
\hline SP4_2 & 0 & 0.08 & 0.32 & 0.51 & 0.65 \\
\hline SP4_4 & 0 & 0.17 & 0.34 & 0.46 & 0.58 \\
\hline SP6_2 & 0 & 0.16 & 0.37 & 0.51 & 0.64 \\
\hline SP6_4 & 0 & 0.18 & 0.32 & 0.43 & 0.52 \\
\hline SP8_2 & 0 & 0.18 & 0.37 & 0.49 & 0.61 \\
\hline SP8_4 & 0 & 0.17 & 0.30 & 0.40 & 0.47 \\
\hline SP12_2 & 0 & 0.20 & 0.34 & 0.46 & 0.54 \\
\hline SP12_4 & 0 & 0.17 & 0.27 & 0.35 & 0.41 \\
\hline SP16_2 & 0 & 0.19 & 0.32 & 0.41 & 0.49 \\
\hline SP16_4 & 0 & 0.16 & 0.25 & 0.32 & 0.37 \\
\hline SP24_2 & 0 & 0.18 & 0.28 & 0.36 & 0.43 \\
\hline SP24_4 & 0 & 0.14 & 0.22 & 0.28 & 0.32 \\
\hline SP32_2 & 0 & 0.17 & 0.26 & 0.33 & 0.39 \\
\hline SP32_4 & 0 & 0.13 & 0.19 & 0.24 & 0.28 \\
\hline \hline
\end{tabular}

Assuming a two 1-D estimator, the first step is to estimate the channel at pilot positions. The simplest technique is the Least Square (LS) estimation, which does not exploit the correlation of the channel across frequency and time [85]. Considering the system model:

$$
Y[n, k]=X[n, k] H[n, k]+N[n, k]
$$

where $X[n, k]$ is the data, $H[n, k]$ is the CFR, and $N[n, k]$ is the AWGN noise at k-th subcarrier of the $\mathrm{n}$-th OFDM symbol, the LS estimation of $H[n, k]$ is obtained as:

$$
\widehat{H}[n, k]=\frac{Y[n, k]}{X[n, k]}=H[n, k]+\frac{N[n, k]}{X[n, k]}
$$


It can be seen that there will be an error introduced by the non-ideal estimation, that will depend on the noise power and pilot boosting. Mean Square Error (MSE) is usually considered as a performance measure of channel estimates, and it is defined as $M S E=E\|H[n, k]-\widehat{H}[n, k]\|^{2}$. Other estimation techniques such as Maximum Likelihood (ML) or Linear Minimum Mean Square Error (LMMSE) provide more accurate estimates. Nevertheless, their complexity is significantly increased as they require knowledge of channel statistics.

The second step in the CFR estimation is the interpolation across the scattered pilot carriers in order to obtain $\widehat{H}$ on data carriers. The interpolation error also affects the estimation accuracy, and so the MSE. In this step, the linear interpolation at data positions provides the lowest complexity. Nevertheless, it involves the poorest performance for channels with high frequency-selectivity, i.e., channels with large delay spread [86]. More accurate estimates can be obtained by applying different smoothing filters, such as Wiener filtering [87].

As it has been shown, there are different aspects regarding the channel estimation implemented at receivers that affects the performance. In addition, when LDM is used, a fine channel estimation is even more crucial because of an additional error, which is described in the next section.

\subsubsection{Channel Estimation in LDM}

LDM mode requires of the CL signal cancellation in order to obtain the EL. If the CL signal has not been properly obtained, a cancellation error appears. This error is known as Cross-Layer Interference (CLI). The CLI also depends on an accurate channel estimation, so that the need of a precise CFR estimation in LDM is even higher than for non-LDM systems. The estimation of the CLI is presented next.

A transmitted LDM signal is assumed to be defined as:

$$
X_{L D M}[n, k]=X_{C L}[n, k]+X_{E L}[n, k]
$$

where $X_{C L}[n, k]$ and $X_{E L}[n, k]$ denote the CL and EL transmitted data at k-th subcarrier of n-th OFDM symbol, respectively. As the power level of the sum of both layers must be normalized, the power level of each layer is defined by the injection level $(\Delta)$, according to:

$$
\begin{aligned}
& P_{C L}=\frac{10^{\frac{\Delta}{10}}}{1+10^{\frac{\Delta}{10}}} \\
& P_{E L}=\frac{1}{1+10^{\frac{\Delta}{10}}}
\end{aligned}
$$


The LDM received signal is:

$$
\begin{aligned}
& Y_{L D M}[n, k]=Y_{C L}[n, k]+Y_{E L}[n, k]= \\
& \quad\left(X_{C L}[n, k]+X_{E L}[n, k]\right) H[n, k]+N[n, k]
\end{aligned}
$$

To decode the EL, the receiver needs to demodulate the CL first (assuming the EL as an additional interference), remodulate it, and cancel it from $Y_{L D M}[n, k]$. From Equation (3.10) the EL can be estimated as:

$$
\widehat{Y}_{E L}[n, k]=Y_{L D M}[n, k]-\widehat{X}_{C L}[n, k] \widehat{H}
$$

where $\widehat{X}_{C L}$ represents the remodulated CL signal. As the EL is intended to provide high capacity services at high SNR, it can be assumed that the CL decoding is error free, that is $\widehat{X}_{C L}[n, k]=X_{C L}[n, k]$. Thus, the EL can be obtained as:

$$
\begin{aligned}
& Y_{E L}[n, k]=X_{C L}[n, k]H[n, k]-\widehat{H}[n, k])+ \\
& X_{E L}[n, k] H[n, k]+N[n, k]= \\
& I_{C L}[n, k]+X_{E L}[n, k] H[n, k]+N[n, k]
\end{aligned}
$$

where $I_{C L}[n, k]$ is the CLI from the CL into the EL, It is calculated as:

$$
\begin{aligned}
I_{C L}[n, k]=X_{C L_{R x}}[n, k](H[n, k]-\widehat{H}[n, k]) & \\
= & =X_{C L_{R x}}[n, k] \Delta_{H}[n, k]
\end{aligned}
$$

From (3.13), as $X_{C L_{R x}}$ and $\Delta_{H}$ can be approximated by Gaussian-distributed random processes and are independent, the CLI can be modelled as a Gaussiandistributed random process [45], with zero mean and power:

$$
P_{C L I}=M S E \cdot P_{X_{C L R x}}=M S E \cdot 10^{\Delta / 10}
$$

It should be observed, that CLI power is proportional to the channel estimation error (MSE) and the CL power. Although it was shown in different literature references [45] that this additional interference is almost negligible in comparison with the EL noise threshold, it has to be taken into account in channel estimation studies. 


\subsection{Methodology and Simulation Setup}

The performance of all the different studies is evaluated by means of physical layer simulations with a validated ATSC 3.0 software simulator. Despite the proposed paper is focused on the optimization of the SP for TDM and LDM modes of ATSC 3.0, the impact of FFT size and TIL length is provided first because of their greater influence on mobility conditions compared to SPs.

The different studies are structured as follows.

A. FFT size and TIL length impact for TDM systems. As these parameters mainly relate to time-varying channels, the results are only obtained for mobile reception. The configurations adopted are:

- Channel model: Typical Urban (TU-6) for Doppler shifts 11, 17, 22, 33, 44, 55, 83, and $111 \mathrm{~Hz}$.

- Pilot configuration: SP3_2 with pilot boosting 2.

- FFT and GI: The 3 FFT sizes (8k, $16 \mathrm{k}$, and $32 \mathrm{k})$. The GIs are extracted from Table 3.2 and assuming SP3_2. To sum up, GI3_512 for 8k, GI5_1024 for 16k, and GI7_2048 for 32k.

- TIL lengths: CTI of 512, 724, and 1024 convolutional rows. They approximately represent 50,100, and $200 \mathrm{~ms}$, respectively.

B. SP density and pilot boosting impact for TDM mode. The configurations assumed are:

- Mobile channel model: TU-6 for Doppler shifts $33 \mathrm{~Hz}$ and $55 \mathrm{~Hz}$.

- Fixed channel models: Rice (DVB-F1) for a common fixed reception, and $0 \mathrm{~dB}$ echo $(50 \% \mathrm{GI})$ as a way of characterizing an SFN.

- TIL length: CTI of 724 rows, equivalent to $100 \mathrm{~ms}$.

- Pilot configuration: SP3_2, SP4_2, SP6_2, SP8_2, SP12_2, SP16 2, SP24 2, SP32 $\overline{2}$ with all the pilot boostings. It can be noted that only SP with $\bar{D}_{y}=2$ are considered, as they are the only ones allowed for $32 \mathrm{k}$ FFT size.

- FFT and GI: 8k, 16k, and 32k for mobile reception. Since the FFT size does not impact on performance for fixed reception, only $16 \mathrm{k}$ 
size has been assumed for this scenario because it allows the use of every SP. The GIs used (extracted from Table 3.2) are summarized in Table 3.5.

C. SP density and pilot boosting impact for LDM mode.

- An LDM $\Delta=4 \mathrm{~dB}$ is assumed. This value distributes the total transmission power as $P_{C L}=71.5 \%$ and $P_{E L}=28.5 \%$.

- The rest of parameters are configured equally as for TDM.

Other parameters common in all the studies are:

- $6 \mathrm{MHz}$ Bandwidth (BW) signal.

- Mobile transmission mode: QPSK 4/15 (data rate of $3.1 \mathrm{Mbps}$ )

- Fixed transmission mode: 64NUC 10/15 (data rate of 23.8 Mbps)

- All simulations are conducted under realistic channel estimation. An LS estimator for the scattered pilot carriers with a two 1-D interpolator is assumed. The interpolation is constituted by a linear time interpolator for obtaining the CFR at data carriers between OFDM symbols and a Wiener frequency interpolator for obtaining the CFR at data carriers of the same OFDM symbol.

- The results are obtained for a Bit Error Rate $(\mathrm{BER})=10^{-4}$.

\subsection{Simulation results}

\subsubsection{FFT size and TIL depth impact in TDM systems}

Figure 3.4 presents the SNR threshold in $\mathrm{dB}$ at different speeds for $8 \mathrm{k}, 16 \mathrm{k}$, and 32k FFT sizes and 50, 100, and $200 \mathrm{~ms}$ TIL lengths. The figure shows that for pedestrian and very high speeds the system performance decreases. In the case of pedestrian reception the performance loss comes from the lack of time diversity, due to the large coherence time. For high speeds, the higher the FFT size the lower the Doppler shift limit, i.e. lower speeds are allowed. In particular, from Equation (3.2), the Doppler shift limits for $8 \mathrm{k}, 16 \mathrm{k}$ and $32 \mathrm{k}$ are 187 $\mathrm{Hz}, 92 \mathrm{~Hz}$, and $50 \mathrm{~Hz}$, respectively. For a carrier frequency $f_{c}=600 \mathrm{MHz}$ these 
Table 3.5: Simulation configurations for SP and Pilot boosting

\begin{tabular}{cccc}
\hline \hline SP & $F F T 8 k$ & $F F T 16 k$ & $F F T$ 32k \\
\hline SP3_2 & GI7 & GI11 & GI9 \\
\hline SP4_2 & GI6 & GI10 & - \\
\hline SP6_2 & GI5 & GI8 & GI7 \\
\hline SP8_2 & GI4 & GI6 & GI6 \\
\hline SP12_2 & GI3 & GI5 & GI5 \\
\hline SP16_2 & GI2 & GI4 & GI4 \\
\hline SP24_2 & - & GI3 & GI3 \\
\hline SP32_2 & GI1 & GI2 & GI2 \\
\hline \hline
\end{tabular}

limits correspond to 335,165 , and $90 \mathrm{~km} / \mathrm{h}$, which approximately coincide with those shown in the figure.

The same figure also shows that the gain introduced by the TIL at pedestrian and vehicular speeds is around $1 \mathrm{~dB}$ for all the FFT sizes. However, when the receiver speed is increased to the ICI-limited zone, the benefits for using longer TIL are reduced.

As a conclusion, if the maximum planned speed is below the Doppler limit, that is, below the ICI-limited zone, the highest TIL length is recommended. In the case of ATSC 3.0, the highest TIL length is 1024 convolutional rows, which represents approximately $200 \mathrm{~ms}$.

\subsubsection{SP and Pilot Boosting impact for TDM systems}

This section studies the impact of SP density and boosting on TDM systems for fixed and mobile reception.

\section{Fixed roof-top scenario in TDM systems}

Figure 3.5 presents the SNR threshold in $\mathrm{dB}$ obtained for the different SPs and pilot boostings for $16 \mathrm{k}$ FFT size.

Pilot density: It can be seen that the impact of the SP density is not significant since the sparsest pattern already fits the minimum required frequency 


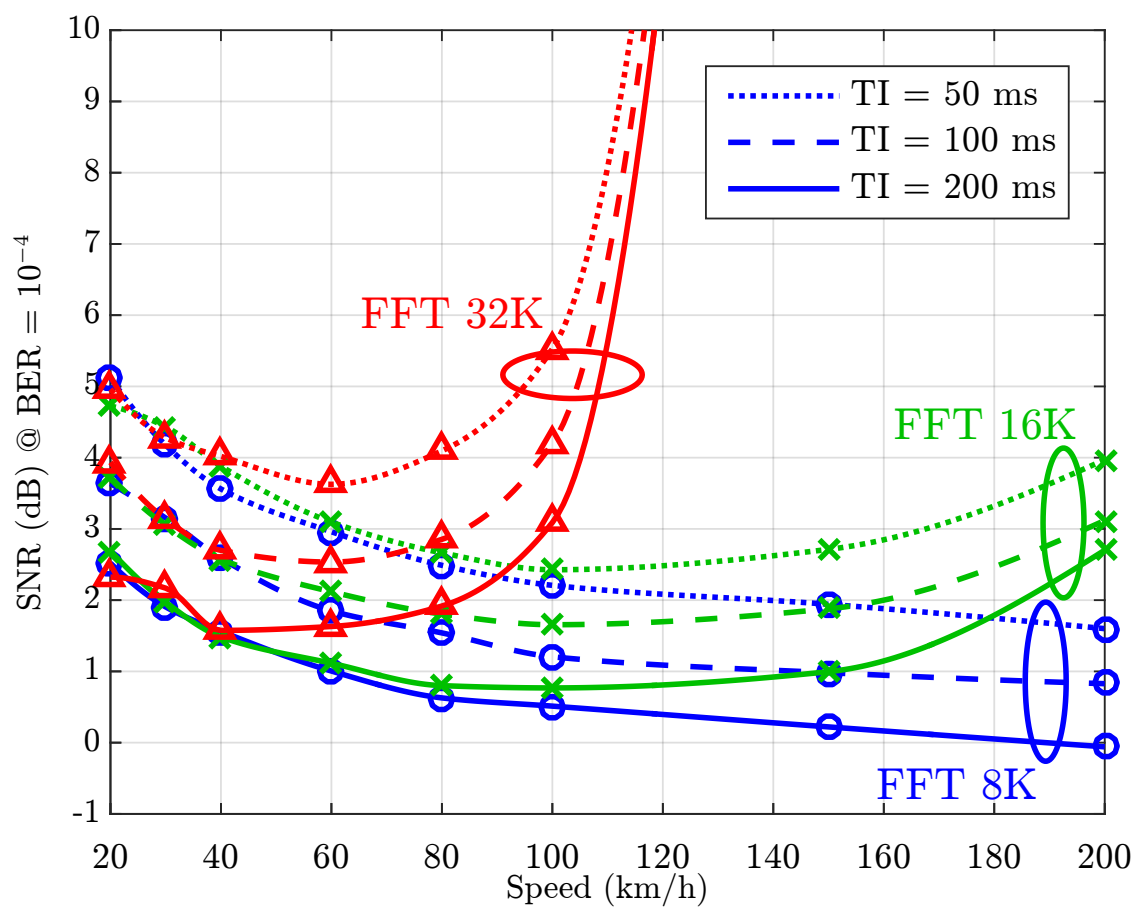

Figure 3.4: SNR threshold in dB for different FFT sizes and TIL lengths at different speeds $\left(f_{c}=600 \mathrm{MHz}\right)$. SP3_2 with pilot boosting 2 was assumed.

separation. Table 3.6 shows that the Nyquist limit of each SP is always larger than the Rice or $0 \mathrm{~dB}$ echo channels delay spreads. Hence, the fact of using denser patterns hardly improves performance, as it provides a more accurate channel estimation but at the same time it enhances the frequency domain noise bandwidth. This trend was also remarked in [88], where it was noted that the only penalty for using a denser pilot pattern is the capacity reduction. Moreover, it can lead to an slightly worse performance for pilot boostings higher than 0 .

Pilot Boosting: It can be seen that as long as pilot boosting is increased, the overall performance decreases. This is because pilot boosting 0 already provides an accurate estimation, so that there is no need to use higher boostings. In such cases the SNR threshold is increased (see Table 3.4) more than the reduction of the channel estimation error. This conclusion is also valid for denser SPs with the same pilot boosting. The sparsest one is sufficiently 
Table 3.6: Delay Spreads for Rice and $0 d B$ echo channels. FFT $16 \mathrm{k}$

\begin{tabular}{ccccc}
\hline \hline Channel & GI & Delay Spread & SP & Nyquist limit \\
\hline Rice & - & $5.42 \mu \mathrm{s}$ & - & $\geq 74 \mu \mathrm{s}$ \\
\hline & GI11 & $296.3 \mu \mathrm{s}$ & SP3_2 & $790 \mu \mathrm{s}$ \\
\cline { 2 - 5 } & GI10 & $263.9 \mu \mathrm{s}$ & SP4_2 & $592 \mu \mathrm{s}$ \\
\cline { 2 - 5 } GI8 & $175.9 \mu \mathrm{s}$ & SP6_2 & $395 \mu \mathrm{s}$ \\
\cline { 2 - 5 } $0 d B$ echo (50\% GI) & GI6 & $111.1 \mu \mathrm{s}$ & SP8_2 & $296 \mu \mathrm{s}$ \\
\cline { 2 - 5 } & GI5 & $74.1 \mu \mathrm{s}$ & SP12_2 & $197 \mu \mathrm{s}$ \\
\cline { 2 - 5 } & GI4 & $55.5 \mu \mathrm{s}$ & SP16_2 & $148 \mu \mathrm{s}$ \\
\cline { 2 - 5 } & GI3 & $37.1 \mu \mathrm{s}$ & SP24_2 & $98 \mu \mathrm{s}$ \\
\cline { 2 - 5 } & GI2 & $27.8 \mu \mathrm{s}$ & SP32_2 & $74 \mu \mathrm{s}$ \\
\hline \hline
\end{tabular}

dense, so that using a denser SP will reduce the SNR more than the channel estimation error.

In summary, it can be concluded that for TDM systems in fixed reception, an sparse SP with the minimum pilot boosting is good enough for obtaining an accurate channel estimation and a good performance.

\section{Mobile scenario in TDM systems}

In the case of mobile reception conditions, the results were obtained for Doppler shifts equal to 33 and $55 \mathrm{~Hz}$. Figure 3.6 illustrates the SNR threshold in dB obtained for all the SP configurations for $33 \mathrm{~Hz}$ Doppler shift.

Pilot density: The fact of using denser SPs can provide meaningful gains with pilot boosting 0 (performance gains from SP32_2 to SP3_2 are 1.2 dB FFT $8 \mathrm{k}, 0.6 \mathrm{~dB}$ for FFT $16 \mathrm{k}$, and $0.3 \mathrm{~dB}$ for FFT $32 \mathrm{k})$. For the rest of the boostings the performance gains by increasing SP density are no longer noticeable $(0.6 \mathrm{~dB}$ FFT 8k, 0.2 dB for FFT 16k, and -0.2 dB for FFT 32k from SP32_2 to SP3_2 approximately). Since the TU-6 channel delay spread is equal to $5 \mu \mathrm{s}$, which is again shorter than the Nyquist limit allowed by each FFT/SP combination assumed, it can be considered that the selection of $D_{x}$ is not critical as well. On the other hand, it can be observed that the performance for FFT $32 \mathrm{k}$ in comparison with the other two FFT sizes is decreased. This performance loss is because $33 \mathrm{~Hz}$ is close to the Doppler limit of this FFT size. 


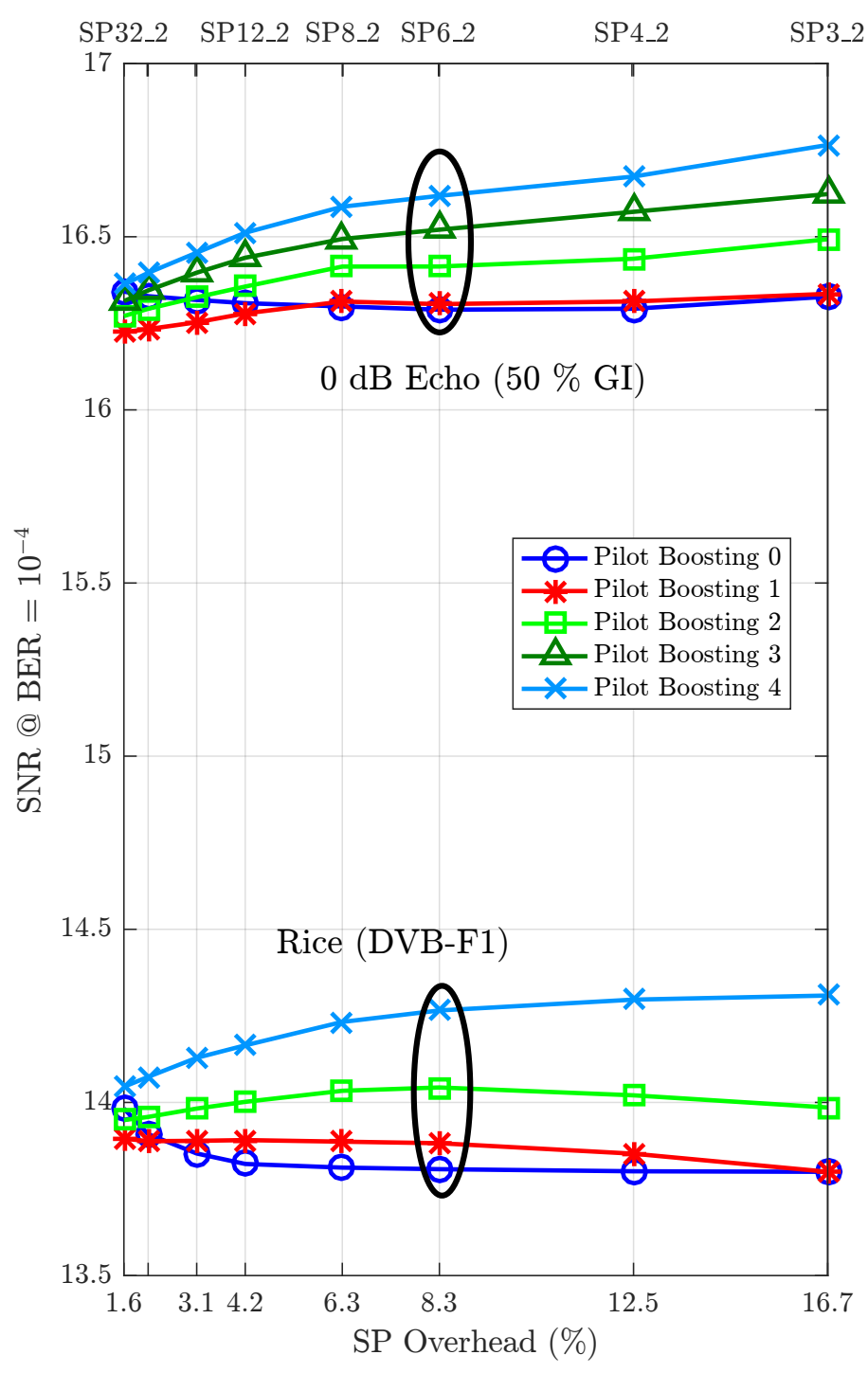

Figure 3.5: SP pattern and boosting impact on SNR performance for fixed reception (TDM). 
Pilot boosting: In this scenario, the best overall performance is achieved with pilot boosting 1 . When pilot boosting is higher than 1 , the same trend as in fixed reception can be observed, increasing pilot boosting decrease overall performance. The same reason given for fixed reception is applied. The reduction on the channel estimation error by using higher pilot boosting is smaller than the required SNR threshold increase. Nevertheless, in this scenario, there is an slight performance gain from pilot boosting 0 to pilot boosting 1 that decreases with the SP density and is independent of the FFT size. Specifically, the performance gains when using boosting 1 instead of boosting 0 are $0.5 \mathrm{~dB}$ for all the FFT sizes when SP32_2 is used, $0.3 \mathrm{~dB}$ when SP16_2 is used, and $0 \mathrm{~dB}$ when SP3 2 is used.

Figure 3.7 illustrates the SNR threshold in dB obtained for all the SP configurations with $55 \mathrm{~Hz}$ Doppler shift. This scenario can be assumed as high speed reception, since $55 \mathrm{~Hz}$ Doppler shift is higher than the Doppler shift limit for FFT $32 \mathrm{k}$. Thus, only $8 \mathrm{k}$ and $16 \mathrm{k}$ FFT sizes are presented. The performance for the two allowed FFT sizes is very similar than to $33 \mathrm{~Hz}$. Hence, the same conclusions can be extracted here.

In summary, the use of pilot boostings 0 or 1 is recommended for mobile reception, provided a dense enough SP is used according to receiver speed. As an specific recommendation, SP12_2 with boosting 1 could be considered the optimum SP because it offers almost the same performance as denser patterns but much less capacity overhead.

\subsubsection{SP and Pilot Boosting impact for LDM systems}

The same studies presented for TDM were done for LDM with an injection level of $\Delta=4 \mathrm{~dB}$. Same conclusions about channel estimation for the CL are expected, because in LDM the SPs are not affected by the EL. However, the impact of CLI on the EL performance should be studied.

\section{Mobile scenario in LDM systems}

Figure 3.8 shows the CL SNR threshold in $\mathrm{dB}$ for a Dopppler shift of 33 $\mathrm{Hz}\left(60 \mathrm{~km} / \mathrm{h}\right.$ at $\left.f_{c}=600 \mathrm{MHz}\right)$ for the three FFT sizes. As expected, the results for mobile reception are almost the same to the ones obtained for TDM systems. Nonetheless, there are some considerations to highlight.

Pilot density: The performance gains by using denser SPs can be obtained for pilot boosting 0 , as in TDM, but these gains are bigger (from SP32_2 to 
FFT 8k

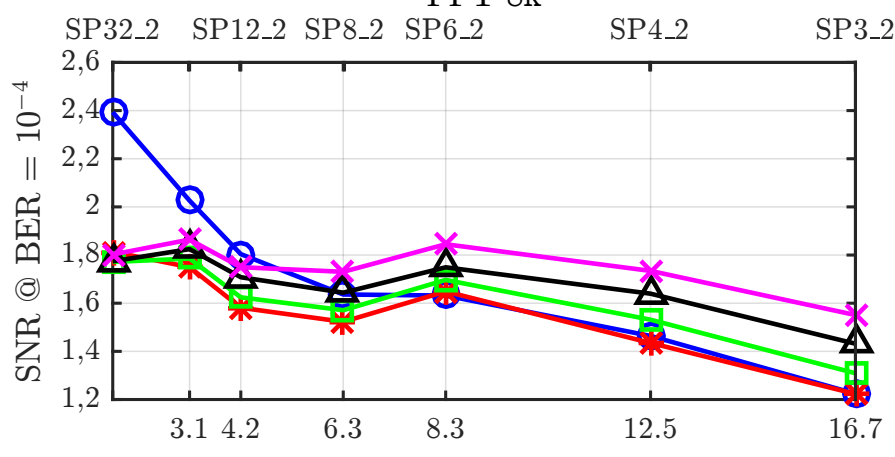

FFT16k
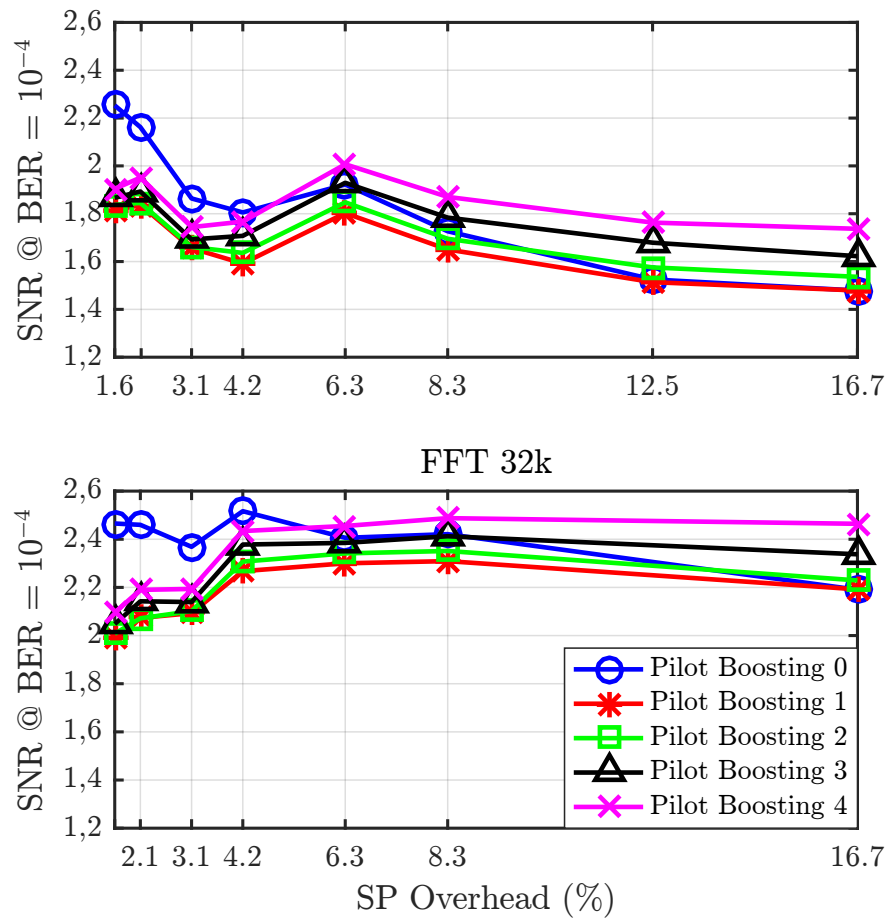

Figure 3.6: SP pattern and boosting impact on SNR performance for mobile reception at $60 \mathrm{~km} / \mathrm{h}$ in TDM systems $\left(f_{c}=600 \mathrm{MHz}\right)$. 

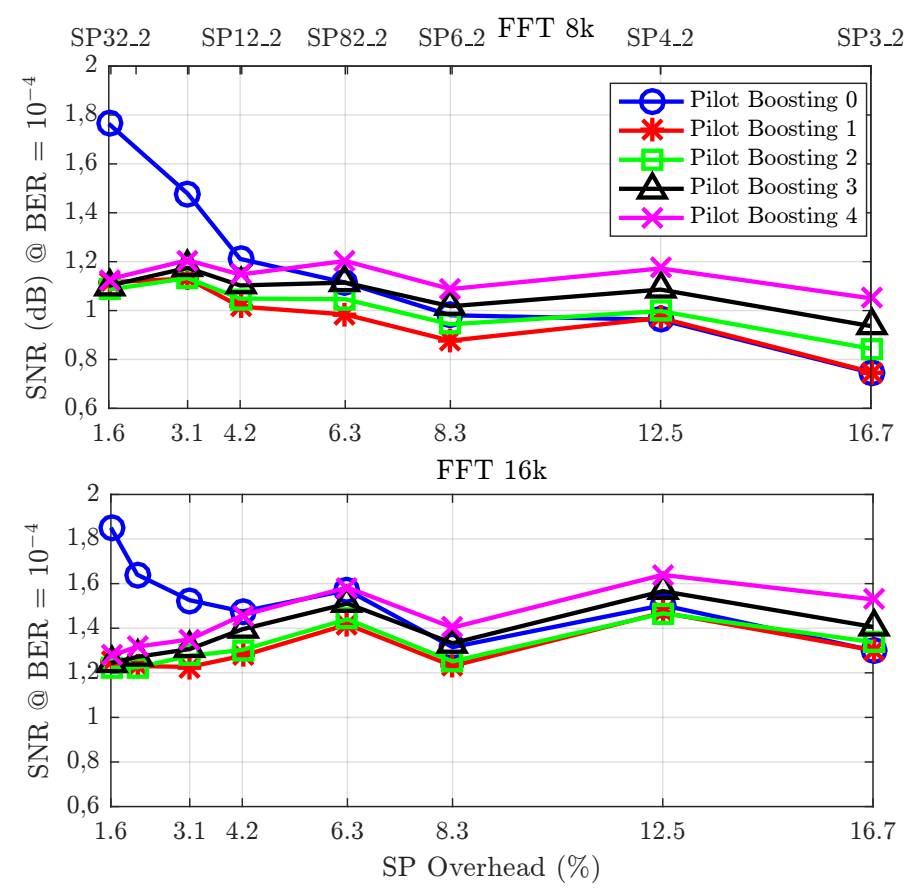

Figure 3.7: SP pattern and boosting impact on SNR performance for mobile reception at $100 \mathrm{~km} / \mathrm{h}$ in TDM systems $\left(f_{c}=600 \mathrm{MHz}\right)$.

SP3_2 the SNR threshold is reduced $1.8 \mathrm{~dB}$ for FFT 8k, $1.1 \mathrm{~dB}$ for FFT 16k, and $\overline{1} \mathrm{~dB}$ for FFT $32 \mathrm{k}$ ). For the rest of the boostings, the performance is practically the same for every SP, as in TDM systems.

Pilot boosting: Again, for sparse SPs, pilot boosting 0 is not recommended. Moreover, with this multiplexing mode, the performance gains from pilot boosting 0 to pilot boosting 1 are increased (the SNR threshold is reduced about $1.5 \mathrm{~dB}$ with SP32_2 for every FFT size). Another conclusion that can be extracted is that the performance of using pilot boosting higher than 1 is not decreased. In this case, the better estimation accuracy obtained by higher boostings compensates not only for $\Delta_{B P}$, but the LDM layers normalization power.

The performance of the CL at $55 \mathrm{~Hz}$ Doppler shift for 8k and 16k FFT sizes is illustrated in Figure 3.9. Again, as it was highlighted in TDM systems, the same conclusions regarding pilot density and pilot boosting performance for 
FFT $8 \mathrm{k}$

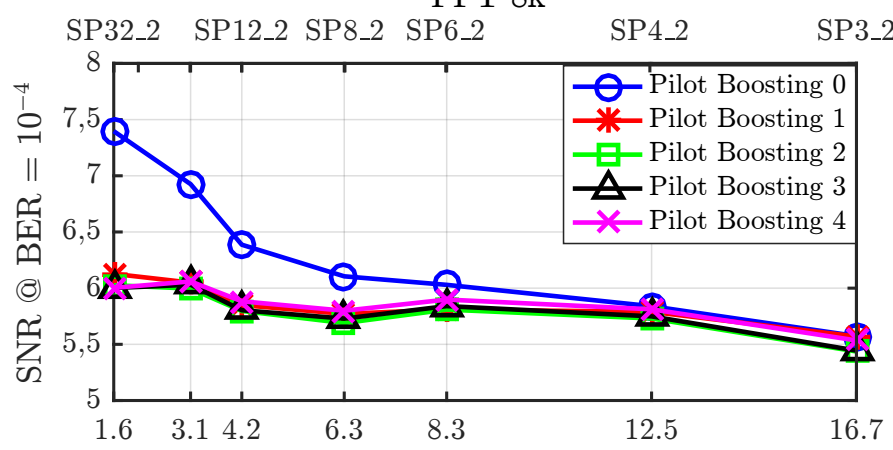

FFT 16k

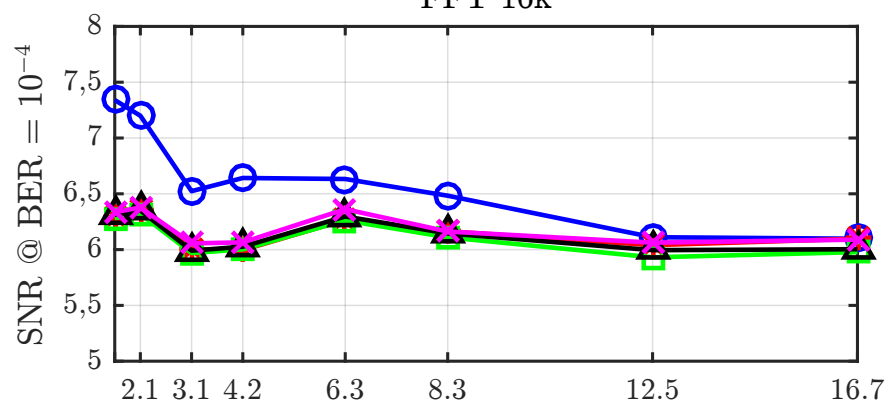

FFT $32 \mathrm{k}$

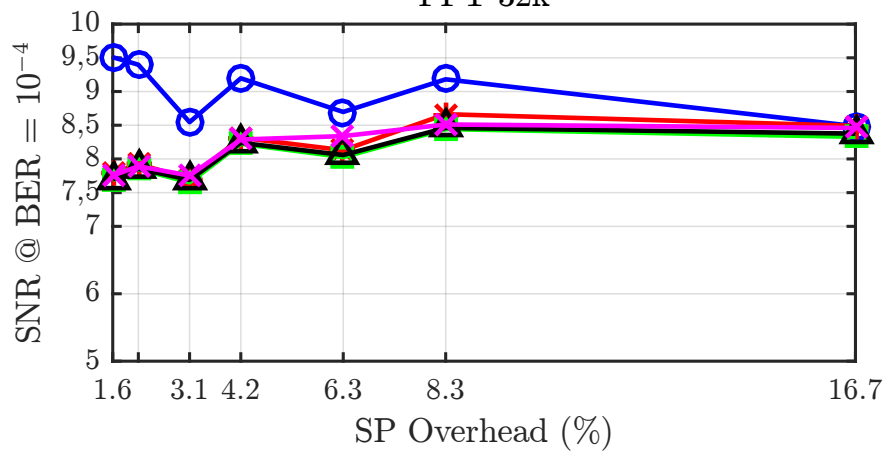

Figure 3.8: SP pattern and boosting impact on SNR performance for mobile reception at $60 \mathrm{~km} / \mathrm{h}(\mathrm{CL}$ LDM, $\Delta=4 \mathrm{~dB})$. 

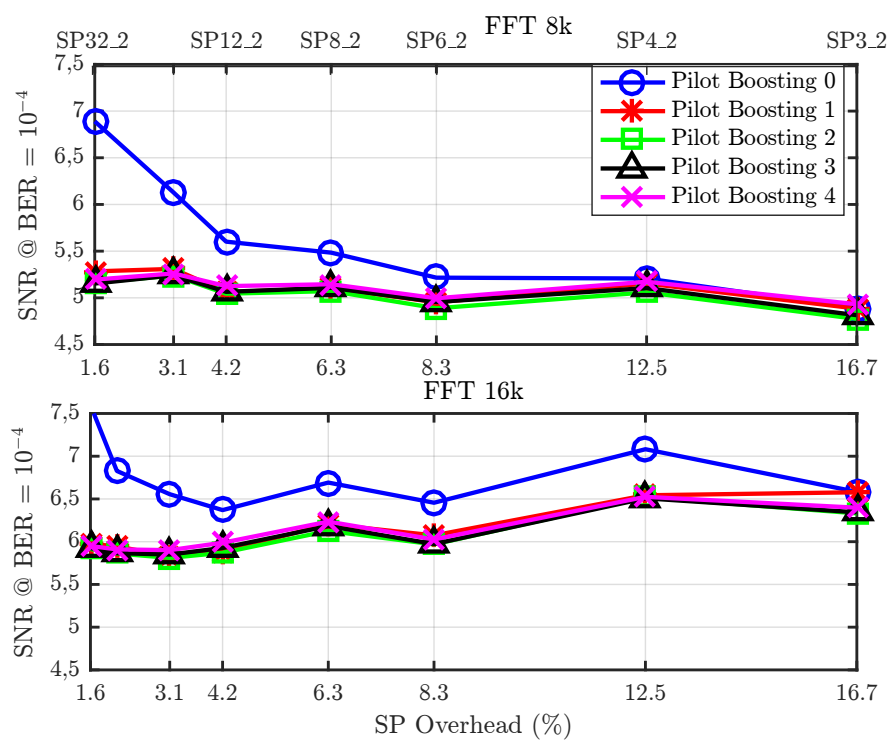

Figure 3.9: SP pattern and boosting impact on SNR performance for mobile reception at $100 \mathrm{~km} / \mathrm{h}\left(\mathrm{CL}\right.$ LDM, $\left.\Delta=4 \mathrm{~dB}, f_{c}=600 \mathrm{MHz}\right)$.

the CL at $33 \mathrm{~Hz}$ Doppler shift, can also be assumed for high speed reception conditions.

\section{Fixed roof-top scenario in LDM systems}

Regarding the EL performance, it can be observed in Figure 3.10 that despite the CLI introduced, the results are very similar to TDM. Apart from the $5.5 \mathrm{~dB}$ SNR threshold increase inherent to LDM with $\Delta=4 \mathrm{~dB}^{2}$. Other considerations that can be highlighted:

Pilot density: As it has been said for the CL, the only difference with respect to TDM systems is that using denser SPs with pilot boosting 0 improves the performance in a greater proportion, because of the performance loss of the sparsest SPs. The impact of increasing pilot density with other pilot boostings is negligible.

\footnotetext{
${ }^{2}$ The Enhanced Layer SNR threshold is approximately $\Delta+10 \log \left(1+10^{-\Delta / 10}\right) \mathrm{dB}$ higher than the SNR without LDM
} 


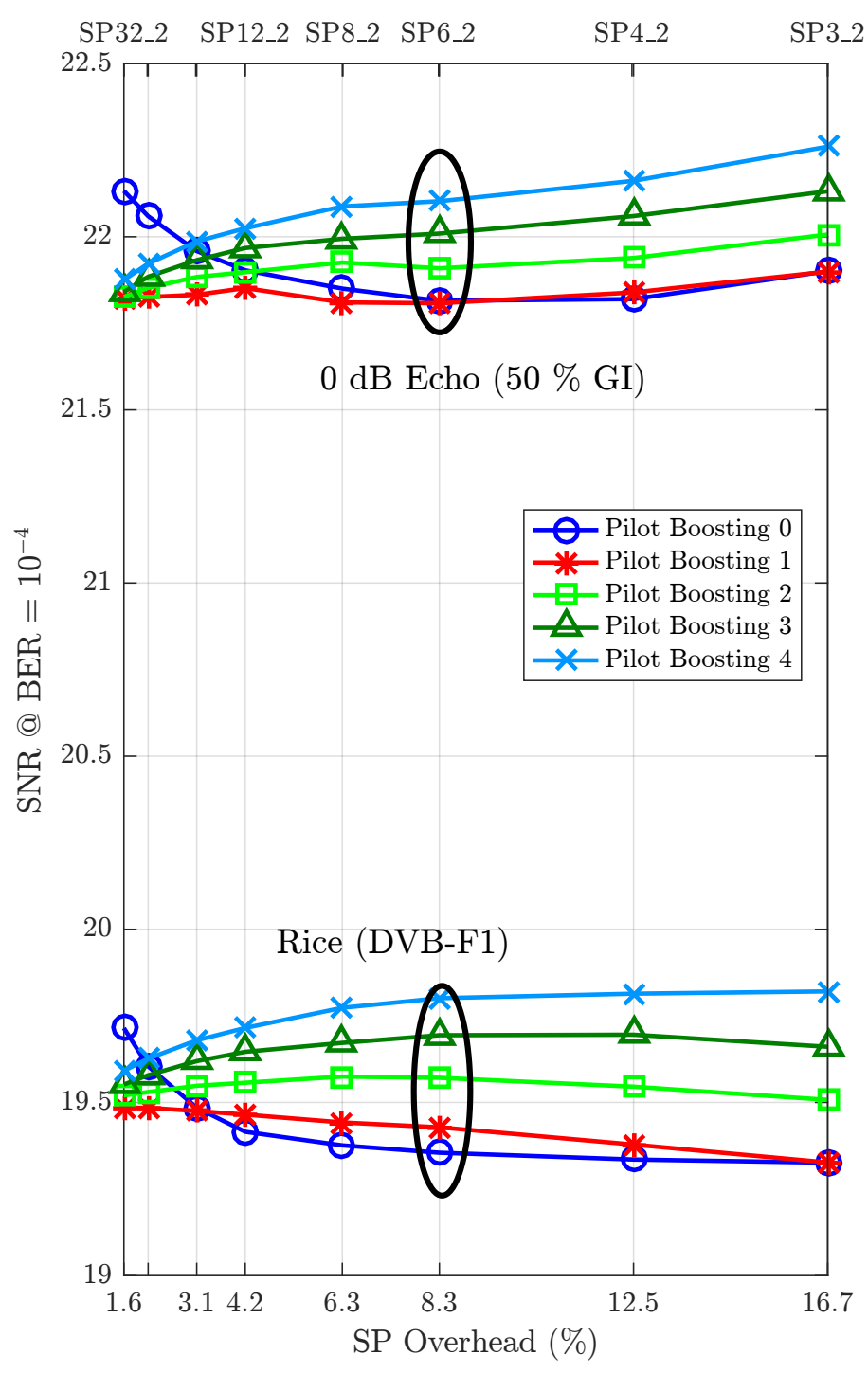

Figure 3.10: SP pattern and boosting impact on SNR performance for fixed reception (EL LDM, $\left.\Delta=4 \mathrm{~dB}, f_{c}=600 \mathrm{MHz}\right)$. 

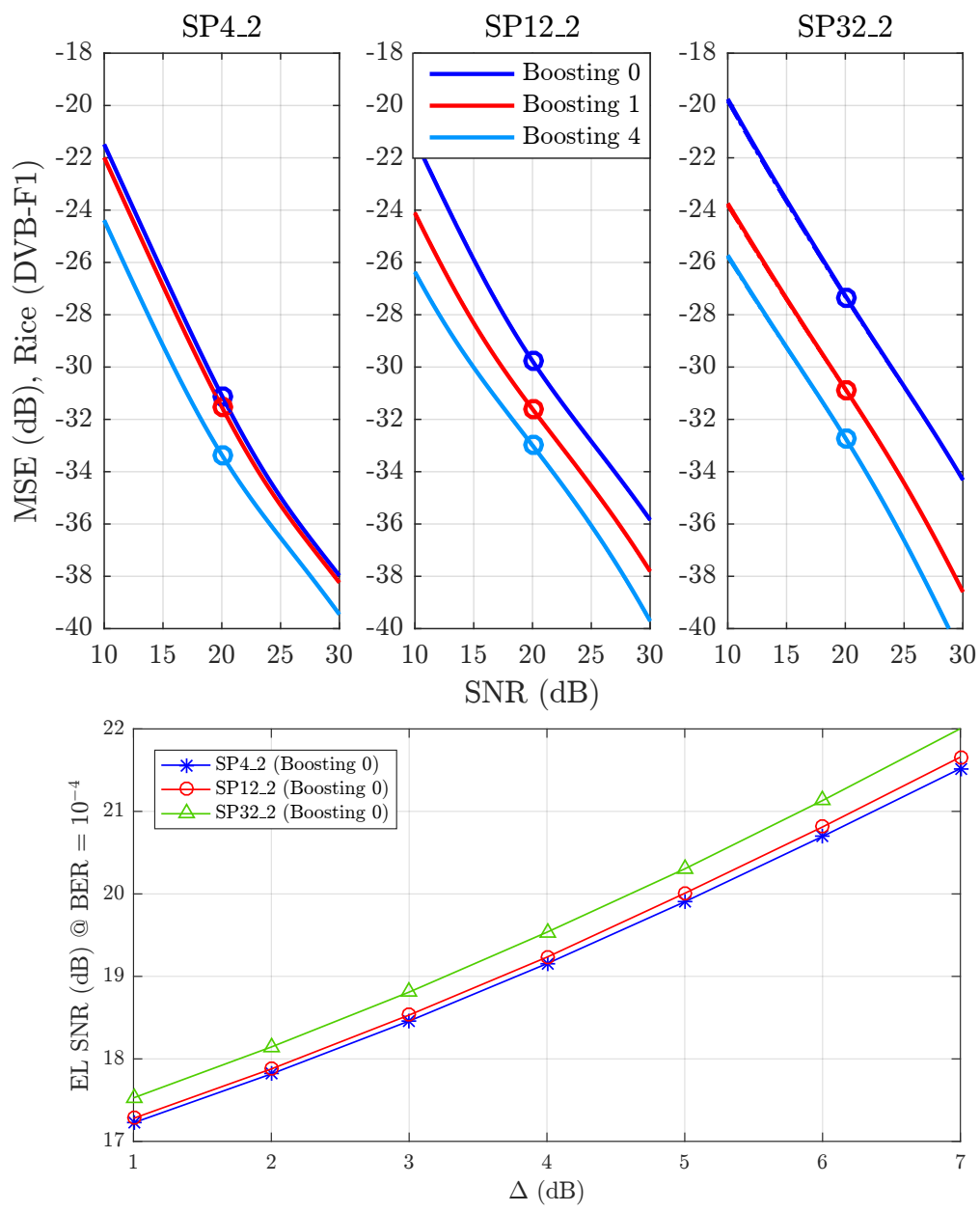

Figure 3.11: CLI depends on the MSE of Channel Estimation (top) and on the $\Delta$ (bottom). Rice fading channel. SP4_2, SP12_2, and SP32_2

Pilot boosting: As long as pilot boosting is increased, the overall performance decreases. This is because pilot boosting 0 already estimates the CFR accurately.

Although the CLI can be considered almost negligible, the main differences in channel estimation of LDM with respect to TDM systems are for the sparsest patterns with boosting 0 . The reason comes from this additional CLI associated with LDM. In (3.14) the CLI was shown to depend on two factors, the 
quality of the channel estimator, i.e. the MSE, and the LDM injection level, $\Delta$. Figure 3.11 shows the MSE for SP4_2, SP12_2, and SP32_2 (top figure) and the EL SNR threshold for different $\bar{\Delta}$ (bottom). It can be observed at the top part of the figure that the highest MSE, and so does, the highest CLI, is introduced by SP32_2 with pilot boosting 0 . In addition, when $\Delta$ is increased, more power is assigned to the CL, so that a higher CLI power is produced. It can be observed at the bottom part of the figure that as long as $\Delta$ is increased the performance gaps between SPs are increased as well.

Given that CL and EL must share the same SP pattern and taking into account the performance presented in previous figures, it is recommended not to use an sparse SP with pilot boosting 0 in LDM systems. This is especially remarkable when $\Delta$ is higher than $3 \mathrm{~dB}$. As a particular recommendation, SP6_2 with boosting 1 could be assumed as an optimum SP because it offers almost the best performance among all density-boosting combinations, but with low capacity overhead.

\subsection{Conclusions}

This paper evaluates the performance of the different pilot configurations allowed in ATSC 3.0 by physical layer simulations under realistic channel estimations. In contrast with DVB-T2, ATSC 3.0 offers up to 16 different scattered pilot patterns (SP), where each one could use up to 5 different pilot boostings. Thus, the selection of the optimum pilot configuration is not as obvious. The studies were done with different fading channels, Rice and $0 \mathrm{~dB}$ echo $(50 \%$ GI) for fixed reception and TU-6 for mobile reception. The studies have been conducted for Time (TDM) and Layered Division Multiplexing (LDM) modes of ATSC 3.0.

From the simulation results obtained for TDM systems, it can be observed that for fixed reception conditions, the use of dense SP is not required, as all of them accomplish with the Nyquist limit. Regarding pilot boosting, the channel can be accurately estimated by using higher values. However, the use of high pilot boostings decreases overall performance. From the simulation results, it is observed that despite the greater accuracy, it is recommended the use of the minimum pilot boosting. Regarding mobile reception, the same conclusion applies for pilot boosting, but a denser SP than for fixed reception is needed. As an specific recommendation, SP12_2 with boosting 1 is proposed as the optimum pilot configuration. The overhead of this SP is only $4.2 \%$, and 
it allows the use of SFNs of distance between transmitters up to $45 \mathrm{~km}$ and $105 \mathrm{~km}$ for 16k FFT size and 32k FFT size, respectively.

On the other hand, LDM introduces a new challenge for broadcasters since its ATSC 3.0 implementation requires that both layers have to share all the waveform parameters, including the SP. Thus, a trade-off for the optimum SP configuration between robustness of the mobile layer and capacity of the fixed layer arises. Taking into account all these considerations, in order to reduce the inter-layer interference because of non-ideal channel estimation, it is recommended not to use an sparse SP with pilot boosting 0 for high injection levels. As a particular recommendation, SP6_2 with boosting 0 could be assumed as an optimum SP. It offers almost the best performance with low capacity overhead.

It should be noted that the real channel estimator assumed was formed by an LS estimator with linear interpolation in time domain and Wiener frequency interpolation. Thus, although this configuration provides results close to real receivers [87], the conclusions highlighted on this paper can vary if a different channel estimator is used. 
Chapter 4

\section{Layered Division Multiplexing with Multi-Radio Frequency Channel Technologies}

E. Garro, J.J. Gimenez, S.I. Park, D. GomezBarquero, IEEE Trans. on Broadcast., vol. 62, no. 2, pp. 365-374, June 2016. The Advanced Television System Committee $(A T S C)$ is to release the next-generation U.S. Digital Terrestrial Television (DTT) standard, known as ATSC 3.0. Layered Division Multiplexing (LDM) is one of the new physical layer technologies included in the standard, which enables the efficient provision of mobile and fixed services by superposing two independent signals with different power levels. ATSC 3.0 has also adopted a novel transmission technique known as Channel Bonding (CB), which splits the data of a service into two sub-streams that are modulated and transmitted over two radio-frequency (RF) channels. This paper investigates the potential use cases, implementation aspects and performance advantages, for combining LDM with channel bonding and also with the MultiRF channel technology Time Frequency Slicing (TFS), introduced in DVB-T2 (as an informative annex) and $D V B-N G H$, which allows distributing the data of a service across two or more RF channels by means of time slicing and frequency hopping. 


\subsection{Introduction}

The ATSC - Third Generation (ATSC 3.0) Digital Terrestrial Television (DTT) standard [13], [26] introduces new transmission techniques with respect to the current state-of-the-art DTT technology, DVB - Terrestrial Second Generation (DVB-T2) [16], to increase system performance and spectrum flexibility. The efficient simultaneous provision of mobile and fixed services to users, as well as an increased throughput to deliver high quality services such as Ultra HighDefinition TV (UHDTV) are primary targets of the new system.

Power-based Layered Division Multiplexing (LDM) [45] is one of such novel technologies. In LDM, the transmitted signal consists of two independent signals (layers) superimposed together by assigning different power to each layer. With this, a robust layer carries service to mobile receivers while a high capacity layer is intended to transmit services to fixed users. LDM can outperform traditional solutions for the delivery of fixed and mobile services based on Time Division Multiplexing (TDM) [36], [58], [59], such as the use of Physical Layer Pipes (PLP)s or Future Extension Frames (FEF) in the T2-Lite profile in DVB-T2 [39], or Frequency Division Multiplexing (FDM), as implemented in Integrated Services Digital Broadcasting Terrestrial (ISDB-T) [43]. With LDM, each layer uses the full Radio Frequency (RF) bandwidth and transmission time, leading to a higher spectral efficiency. This additional gain can be translated into an increased robustness (or coverage gain) for the same service data rate, or a capacity gain [50]. The implementation of this technique requires increased complexity. LDM mobile receivers can be really simple since they only demodulate the robust layer. Receivers decoding the high capacity layer require the previous cancellation and removal of the robust layer. Furthermore, the implementation of LDM in ATSC 3.0 has been limited so that many components are shared between the two layers [51]. On the other hand, the optimum transmission parameters cannot be independently selected per layer, what drives a trade-off between robustness and capacity.

Additional spectral efficiency increase by Multi-Radio Frequency Channel (MultiRF) channel technologies was also discussed during ATSC 3.0 standardization process. In particular, two MultiRF channel variants were evaluated: Channel Bonding (CB), which basically consists of splitting service data across two RF channels [32], [33], and Time-Frequency Slicing (TFS) [89], that transmits data in a slot-by-slot manner by frequency hopping across an RF-Mux of two or more RF channels (in practice, up to 6) [89]. The main advantages of these two techniques are, basically, capacity and coverage gains. $\mathrm{CB}$ enables services that exceed the data rate offered by a single 
$\mathrm{RF}$ channel. Moreover, it can also provide advantages in combination with Scalable HEVC (SHVC) and LDM [90]. Both TFS and CB also lead to an almost ideal Statistical Multiplexing (StatMux) since it is performed with a large number of services. Improved RF performance can be exploited by means of an increased frequency diversity potentially over hundreds of $\mathrm{MHz}$ by using inter-RF frequency interleaving. This can be translated into a coverage gain for the reception of all services within a RF-Mux, since the reception of a service does not only depend on the quality conditions of a single RF channel. A uniform distribution of the encoded data across two RF channels might allow the recovery of data even when one of the RF channels is corrupted as long as a proper code rate is selected. By similar mechanisms, an increased robustness against interferences is also feasible, which may allow for reducing frequency reuse factor and thus increasing network spectral efficiency [91]. Regarding implementation, $\mathrm{CB}$ requires of two tuners at the receiver, each one fixed on a RF channel while the reception with TFS can be performed, under certain circumstances, using a single tuner.

LDM and MultiRF channel technologies have never been implemented in a terrestrial broadcasting standard and their joint performance is not known. This paper presents the potential use cases of combining LDM and CB or TFS. It evaluates the performance of the joint implementation by means of physical layer simulations and analyses the main implementation aspects at both transmitter and receiver sides.

The paper structure is as follows: Section 4.2 details the main characteristics of LDM, CB and TFS. Section 4.3 evaluates the possible use cases of a joint implementation of LDM with CB and TFS. The main transmitter and receiver implementation aspects are analysed in Section 4.4. Section 4.5 describes the methodology and the simulation setup followed for performance evaluation. The simulation results of LDM with MultiRF channels are presented in Section 4.6. Finally, conclusions are summarized in Section 4.7.

\subsection{Overview of Technologies}

\subsubsection{Layered Division Multiplexing (LDM)}

The concept of LDM, formerly known as Cloud Transmission (CloudTxn) [49], involves the superposition of multiple signals, with different transmit power levels, forming a multi-layer signal. ATSC 3.0 defines LDM with only two layers. The top layer, known as CL, is the most robust one as it can be 


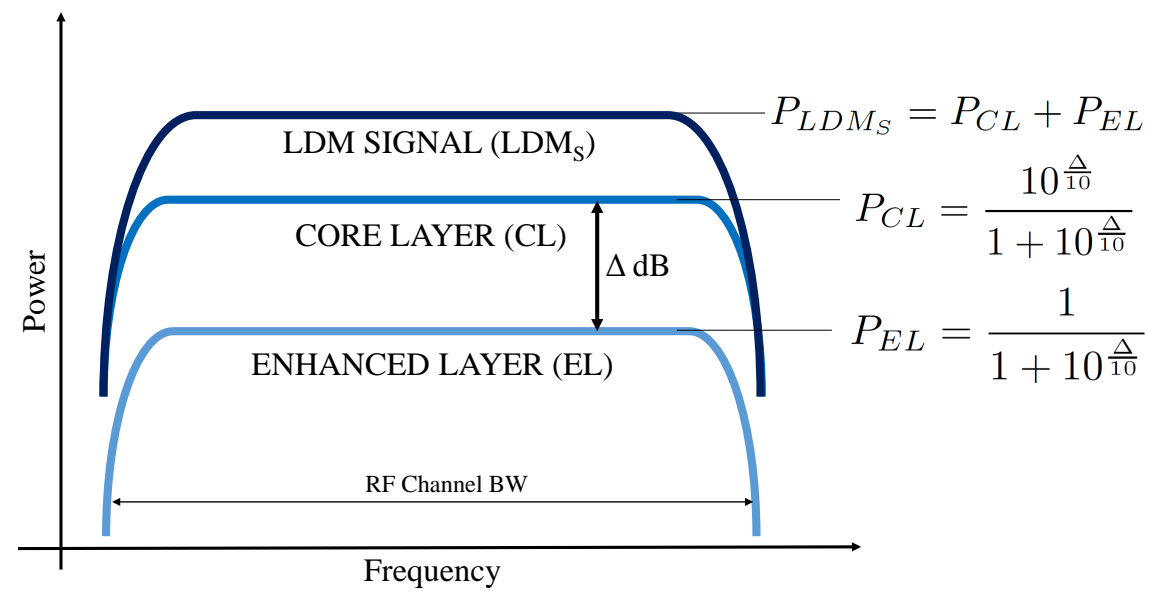

Figure 4.1: LDM Concept. CL and EL sharing the same RF channel by different power assignment. EL is inserted $\Delta \mathrm{dB}$ below CL level.

configured even with a negative Signal-to-Noise Ratio (SNR) threshold [79]. The lower layer, EL, is set with a high capacity (less robust) mode so that it can be used to provide high data rate services to fixed roof-top receivers. The so-called $\Delta$, is the parameter that defines the ratio between the power assigned to the upper and lower layer. As long as $\Delta$ is increased, more power is assigned to the CL and less to the EL, and viceversa. This is directly related with the SNR of both layers. At the receiver side, the EL is demodulated once the CL has been demodulated, cancelled and removed from the received signal.

As it can be seen in Figure 4.1, each layer passes through an independent Bit-Interleaved Coded Modulation (BICM) module, so they can be configured with different Modulation and Coding Rate (MODCOD) parameters. However, several restrictions have been imposed in ATSC 3.0 to limit the receiver complexity. The layers are combined together before the time interleaver, so they share the same Time Interleaver (TIL), as well as the same Orthogonal Frequency-Division Multiplexing (OFDM) waveform parameters: Fast Fourier Transform (FFT), Guard Interval (GI), and Pilot Pattern (PP) scheme. As a result, there is a trade-off when configuring the common transmission parameters for the two layers between the optimum CL and EL parameters:

- A low carrier spacing, a low-dense PP, and a low TIL depth should be chosen to reduce the overheads due to GI and PP and the demodulation latency for fixed service (EL) receivers. 

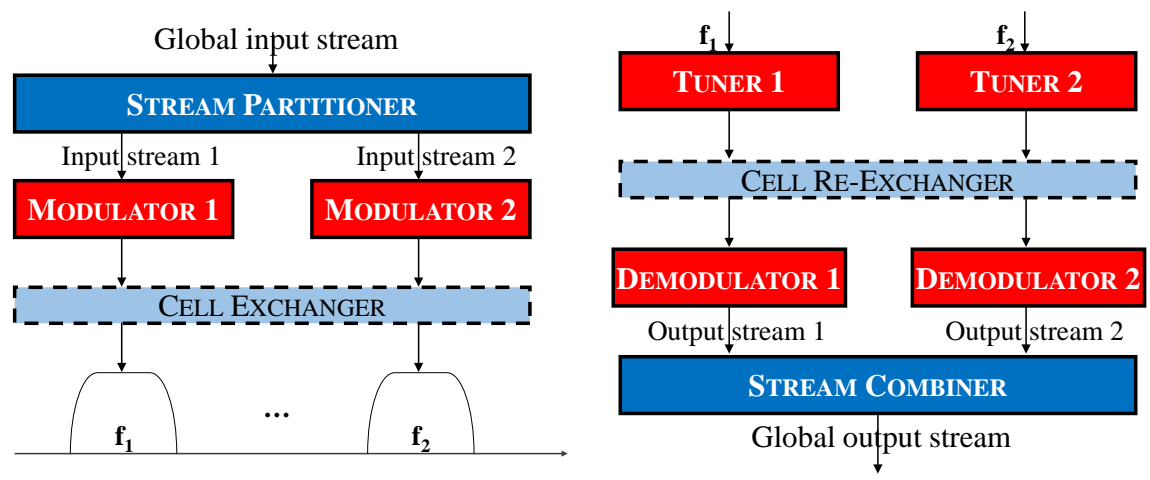

Figure 4.2: $\mathrm{CB}$ transmitter (left) and receiver (right) block diagrams. The cell exchanger and re-exchanger are by-passed with Plain CB and active with SNR averaging CB.

- A high carrier spacing, dense PP, and a larger TIL depth are recommended to deal with fading effects and to avoid Inter-Carrier Interference (ICI) caused by Doppler shift for the mobile service (CL).

Considering these common parameters, when the waveform is configured to favour fixed reception, the CL has a penalty in mobility performance. The lack of an optimum transmission configuration for the CL can be partly compensated by a very robust MODCOD, even with negative SNR threshold. In ATSC 3.0, code rates $2 / 15,3 / 15$, and $4 / 15$ provide a negative SNR threshold (-5.7 dB, $-3.7 \mathrm{~dB}$, and $-2.2 \mathrm{~dB}$ respectively) for Rayleigh channel using Quadrature Phase-Shift Keying (QPSK) modulation [55].

\subsubsection{Channel Bonding (CB)}

CB enables the bundling of two standard-bandwidth RF channels. Basically, the process splits the data of a high-capacity stream into two sub-streams that are modulated and transmitted each one in a different RF channel. At the receiver, a simultaneous demodulation of the $\mathrm{RF}$ channels takes place by means of two independent tuners. The demodulated streams are combined back to create the original single data stream. The RF channels do not necessarily need to be adjacent to each other, thus allowing the reception of channels in different bands e.g. Very-High Frequency (VHF) and Ultra-High Frequency (UHF).

ATSC 3.0 defines two operation modes for CB. Figure 4.2 illustrates the transmitter and receiver block diagrams for both $\mathrm{CB}$ modes. The basic mode is known as Plain $C B$, which enables doubling the transmission of services that 


\begin{tabular}{|c|c|c|c|c|c|c|c|c|c|c|c|c|c|c|c|c|c|}
\hline \multirow{5}{*}{ 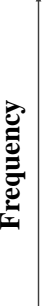 } & \multicolumn{9}{|c|}{ non-TFS Frame } & \multicolumn{8}{|c|}{ TFS Frame } \\
\hline & $\mathrm{RF}_{4} \mid \frac{\mathrm{a}}{2}$ & & 8 & 7 & 8 & 7 & 8 & 7 & 8 & $\mathrm{RF}_{4}$ & 4 & 5 & 6 & 7 & 8 & 1 & 2 \\
\hline & $\mathrm{RF}_{3}$ & 5 & 6 & 5 & 6 & 5 & 6 & 5 & 6 & $\mathrm{RF}_{3}$ & 6 & 7 & 8 & 1 & 2 & 3 & 4 \\
\hline & $\mathrm{RF}_{2}$ & 3 & 4 & 3 & 4 & 3 & 4 & 3 & 4 & $\mathrm{RF}_{2}$ & 8 & 1 & 2 & 3 & 4 & 5 & 6 \\
\hline & $\mathrm{RF}_{1}$ & 1 & 2 & 1 & 2 & 1 & 2 & 1 & 2 & $\mathrm{RF}_{1}$ 䅧 1 & 2 & 3 & 4 & 5 & 6 & 7 & 8 \\
\hline
\end{tabular}

Figure 4.3: Non-TFS (left) and TFS (right) transmission with 4 RF channels and 8 services.

exceed single RF channel throughput. The second operation mode, known as $S N R$ averaging, exploits inter-RF frequency interleaving across the two RF channels, improving transmission robustness [32]. An additional block, the cell exchanger, is employed to ensure an even distribution of data across two RF channels. Cell exchanger distributes the odd and even cells of each Forward Error Correction (FEC) codeword in each RF channel respectively. The reverse operation takes places at the receiver to recover data.

\subsubsection{Time Frequency Slicing (TFS)}

TFS was already introduced as not-normative annex in DVB-T2 and was fully adopted in the Digital Video Broadcasting - Next Generation Handheld (DVB-NGH) specification. TFS distributes the data of each service across two or more RF channels by means of time slicing and frequency hopping. Figure 4.3 illustrates the transmission of services over $4 \mathrm{RF}$ channels in a traditional way and with TFS. FEC codewords of a service are time interleaved, divided into slots and sequentially transmitted over multiple RF channels. Service data recovery is performed by means of frequency hopping over the different channels within the RF-Mux.

Ideally, in order to exploit the extended frequency diversity, each FEC codeword should be evenly split and sent across the RF-Mux. This distribution is achieved by the TIL and a proper framing, so each data-slot containing time-interleaved data of the desired service suffers different SNR conditions according to the RF channels whereby it is received. At the receiver, TFS can be performed with a single tuner provided there is a gap time for tuning between slot boundaries and the tuner is fast enough for seamless reception. The inclusion of these time gaps introduces overheads which can limit the peak data rate of the services. 
Table 4.1: Potential gains of LDM and MultiRF use cases

\begin{tabular}{cccc}
\hline \hline Gain & $\begin{array}{c}L D M+C B \\
\text { (both layers) }\end{array}$ & $\begin{array}{c}L D M+C B \\
(E L \text { only) }\end{array}$ & LDM+TFS \\
\hline Increased Data-Rate & $\checkmark$ & $\checkmark$ & \\
\hline StatMux Gain & $\checkmark$ & $\checkmark$ & $\checkmark$ \\
\hline Increased RF-Performance & $\checkmark$ & & $\checkmark$ \\
\hline \hline
\end{tabular}

\subsection{Use Cases for LDM and MultiRF Channel}

This section describes the potential use cases for a joint MultiRF and LDM implementation. Table 4.1 collects the most relevant use cases and the related advantages that can be exploited.

The most complete combination is the transmission of both LDM layers with CB using the SNR averaging mode with two RF channels in the same frequency band. This mainly allows doubling the service data rate of each layer, an enhanced StatMux as well as improving transmission robustness by inter$\mathrm{RF}$ frequency interleaving. The same transmission mode for both LDM signals is compulsory in this use case, to ensure an appropriate cell exchanging procedure.

The selected CB use cases implement the same transmission mode for both LDM signals or just the EL signal and consider the allocation of the RF channels in the same frequency band in order not to excessively increase receiver complexity. Note that the integration of two different types of antenna on mobile receivers is especially tricky in the case of VHF antennas.

With LDM, CB can also be implemented just in the EL. Implementing CB for the CL would increase mobile receiver complexity since two tuners will be required. When $\mathrm{CB}$ is implemented in the EL, Plain $\mathrm{CB}$ is the only mode allowed, since SNR averaging would exchange the cells of two independent CL streams, which is not desired. If CB is implemented on both layers, the CL sub-streams are no longer independent and form part of a stream that is partitioned. In such case, mobile receivers should implement two tuners.

With TFS, LDM must be performed in both layers what enables inter-RF frequency interleaving as well as StatMux gains.

The most important gains that can be obtained with these combinations are explained below. 


\subsubsection{Increased Peak Service Data-rate}

This advantage can only be obtained when CB is employed since it allows the simultaneous reception of data from two different RF channels. This implementation would allow the transmission of services that exceed the data rate of a single RF channel.

In a classical single RF channel LDM transmission considering a commonly used MODCOD combination QPSK 4/15 for the CL and 64Quadrature Amplitude Modulation (QAM) 10/15 for the EL, the capacities of each layer would be $2.5 \mathrm{Mbps}$ and $20 \mathrm{Mbps}$ respectively. If either Plain CB or SNR Averaging are performed over the two LDM layers, their data rates can be doubled (5 Mbps for CL, and $40 \mathrm{Mbps}$ por EL).

According to [92], and considering that about half data rate is required with High Efficiency Video Coding (HEVC) compared to H.264 [93], HD720p and HD1080p services would require around $2.5 \mathrm{Mbps}$ and $5 \mathrm{Mbps}$ respectively. In such case, the CL with CB could transmit a HD1080p service instead of a HD720p service.

\subsubsection{StatMux Gain}

Both LDM layers could exploit StatMux gain when using CB and TFS. Table 4.2 depicts an illustrative example of the feasible gains for different video services considering the StatMux gain values ${ }^{1}$ for HD services in H.264. The same StatMux gain values are assumed for HEVC coding. The capacities of the CL and EL are, again, 2.5 Mbps (QPSK 4/15) and 20 Mbps (64QAM 10/15). The data rate of the HD services were already introduced in the previous subsection. A 4K UHDTV service using HEVC is considered to require $15 \mathrm{Mbps}$. It can be seen that the most important gains are achieved with HD1080p service in the EL. The StatMux gain increases with the number of RF channels, with significant gains for $6 \mathrm{RF}$ channels. Thus, the most important gains are expected with TFS, thanks to the aggregation of more than 2 RF channels.

\footnotetext{
${ }^{1}$ According to [83], the StatMux gain for HD H.264 is around 15\% for 3-4 programmes, $30 \%$ for 9-12 programmes, and $32 \%$ for $18-24$ programmes
} 
Table 4.2: StatMux gain in number of services for LDM and MultiRF

\begin{tabular}{ccccc}
\hline \hline Layer & Type of service & 2 RF & $4 R F$ & $6 R F$ \\
\hline$C L$ & HD720p @ 2.5 Mbps & 0 & 0 & 1 \\
\hline$E L$ & HD1080p @ 5 Mbps & 2 & 4 & 7 \\
\hline$E L$ & 4K UHD @ $15 \mathrm{Mbps}$ & 0 & 1 & 2 \\
\hline \hline
\end{tabular}

\subsubsection{Increased RF Performance}

CB and TFS could offer an increased RF performance by extending the frequency interleaving across multiple RF channels, so that an increased frequency diversity is achieved. CB can offer this increased RF performance by employing SNR averaging mode between the two RF channels. Furthermore, the use of TFS could provide a higher frequency diversity by the availability of using more than 2 RF channels.

As it was described in Section 4.2.1, the two layers share the same TIL, FFT, GI, and PP in order to limit receiver complexity. As a result, there is a trade-off in the selection of these parameters for optimum mobile or fixed reception. For mobile reception, it is desirable that the interleaving duration is longer than the channel coherence time, which is inversely proportional to the Doppler spread [22]. In the case of pedestrian reception when deep fading occurs, the coherence time would be large, and, thus, the required time interleaving duration should be high. However, this is not always possible due to the limited amount of memory at the receiver for Time De-Interleaver (TDIL) as well as due to the increase latency required, which would affect the performance because of higher zapping times.

For fixed reception, the most important degradation comes from the existing imbalances between RF channels [94]. Inter-RF frequency interleaving averages these SNR imbalances thus harmonizing the coverage of the RF channels whereby the services are transmitted. 


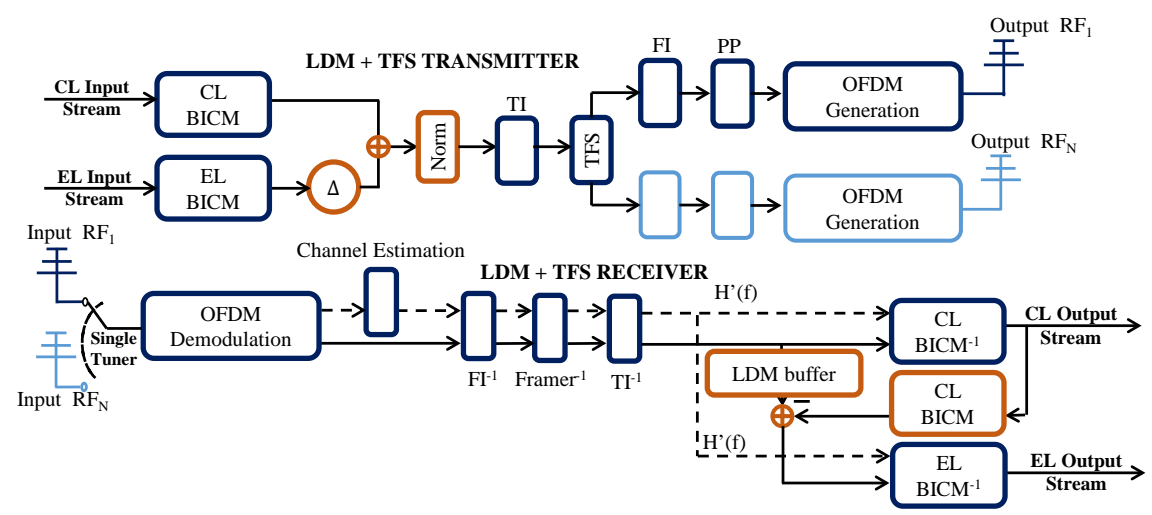

Figure 4.4: LDM + TFS transmitter and receiver block diagrams. At the transmission, CL and EL Input streams pass through independent BICM modules. Then, they are aggregated by injecting the EL $\Delta$ dB below CL. Next, TIL and TFS Framer are executed over the LDM signal in order to transmit it across the $\mathrm{N}$ different RF channels of the RF-Mux. At the receiver, the tuner hops among the RF channel frequencies, demodulating first the CL stream, and if it is desired the EL stream by the LDM cancellation process.

\subsection{Implementation Aspects of LDM with MultiRF Channel Technologies}

\subsubsection{LDM with TFS}

The implementation of TFS for both layers is the only possible solution given that the LDM layers are combined before the TIL and TFS framer [95]. The joint LDM and TFS transmitter and receiver block diagrams are illustrated in Figure 4.4. The two LDM layer streams (CL Input Stream and EL Input Stream) pass through different BICM modules (CL BICM and EL BICM). They are then aggregated by injecting the EL $\Delta \mathrm{dB}$ below CL. The distribution of the two layers aggregated, namely the LDM signal, across the $\mathrm{N}$ RF channels is handled by the TIL and the TFS framer. At the receiver, the tuner hops among the N RF channels in the RF-Mux. The received signal is then demodulated in order to first get the CL stream. The remodulation and cancellation of the CL is also performed if it is desired to receive the EL stream.

TFS requires an even distribution of data across the RF channels, which is achieved by means of time interleaving and a proper framing. With LDM in ATSC 3.0, the TIL is configured according to the size and the number of 
cells of the CL FEC codewords regardless of the EL FEC codewords. Thus, if CL and EL number of cells are different it may happen that they are not equally and evenly spread across the RF-Mux, compromising the expected performance of the EL. The correct TFS operation on the EL also depends on the time interleaver scheme employed. The TIL schemes that can be selected in ATSC 3.0 are:

- A sheer convolutional interleaver (CI) in Single - PLP (S-PLP) [30].

- A hybrid TIL constituted by a joint cell and a twisted block interleaver (BI) for intra-frame TIL, when there are Multiple PLP (M-PLP) [30].

Figure 4.5 presents the TIL output for the CL and EL cells of a frame that are transmitted in RF1 channel when the RF-Mux is composed of 4 RF channels. It is assumed that the EL constellation order is 3 times that of the CL. Each CL FEC codeword involves 12 cells, so there will be 4 cells per EL FEC codeword. Thus, there are 3 times more EL FEC codewords per frame than CL FEC codewords. In this example, there are 8 CL FEC codewords and 24 EL FEC codewords. This illustrative example can be considered as a simplified version of the LDM MODCOD distribution of CL QPSK 4/15 - EL 64QAM 10/15 with a FEC codeword length of 64800 bits. The cells are column-wise written in the TIL matrix. The cells are read-out in a different way according to each TIL. Additionally, the DVB-NGH BI is also shown for comparison, since it is used for TFS operation in DVB-NGH [96].

The sequences to the RF1 channel show that the TIL that reaches the best even distribution of cells is the hybrid cell and twisted block interleaver, where almost one cell of each EL FEC codeword is transmitted.

At the receiver side, the critical point with TFS is tuning operation. TFS reception is possible with a proper scheduling at the transmitter which allocates time gaps between consecutive data slots to enable tuning operation. These time gaps create overheads that restrict peak service data rate. These time margins must include tuning operations (Automatic Gain Control (AGC) + Phase Locked Loop (PLL)) and channel estimation in the time domain (time interpolation among pilot carriers at the start and end of each slot). The necessary time margin for frequency hopping time $\left(t_{h o p}\right)$ is calculated by equation (4.1), where $t_{\text {tuning }}$ depends on the signal bandwidth, FFT, and GI. $t_{C h E}$ depends on the number of symbols required for time interpolation in a scattered PP $\left(D_{Y^{-}} 1\right)[89]$. 

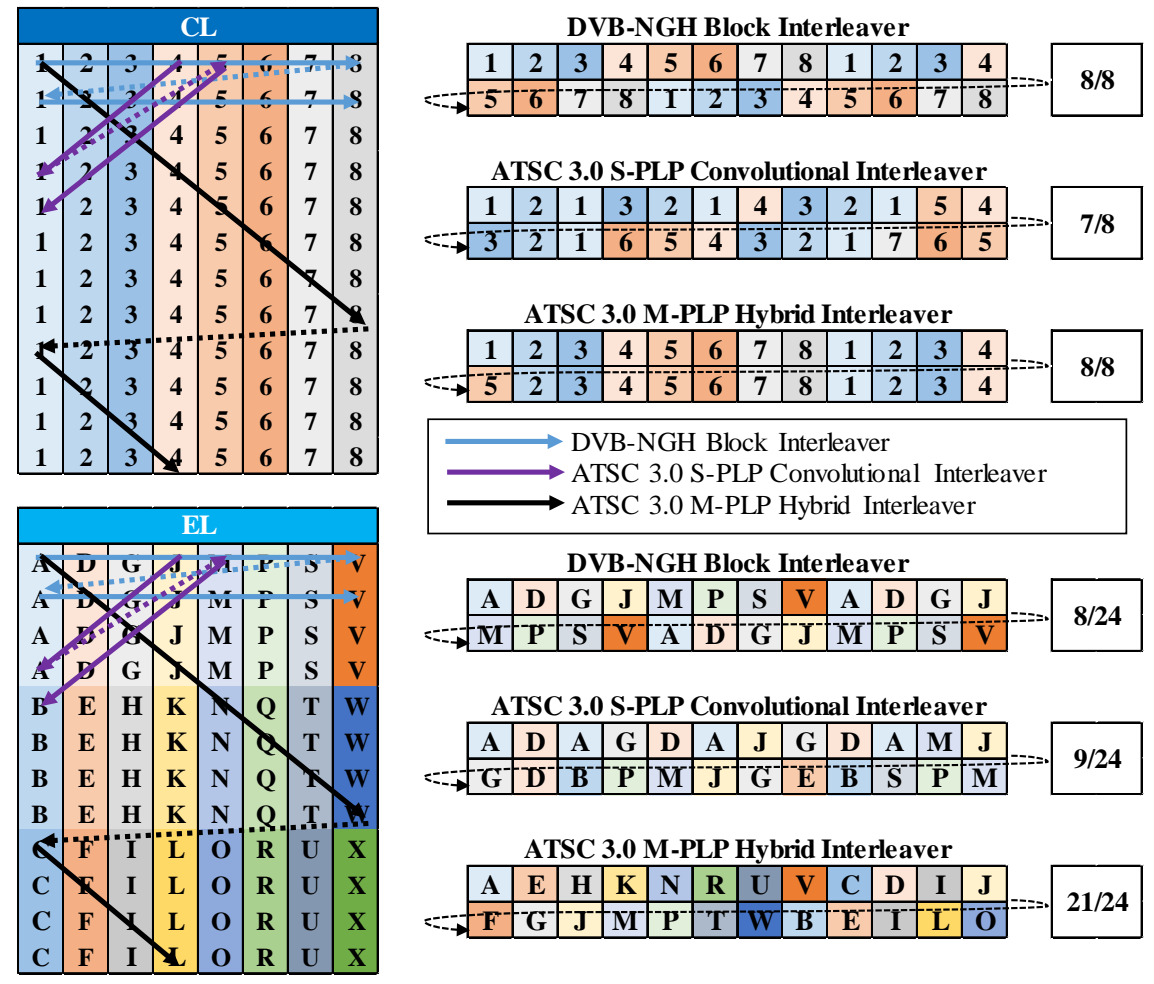

Figure 4.5: Left part of the figure, CL and EL frame matrices composed by 8 FEC codewords of 12 cells (in numbers) and 24 FEC codewords of 4 cells (in letters) respectively. Right part, CL and EL cell output sequences to RF1 of a RF-Mux of 4 RF channels with the different TIL read-out wise schemes. Cells of the 8/8 CL FEC codewords, and 21/24 EL FEC codewords are transmitted with ATSC 3.0 Hybrid TIL. ${ }^{2}$

$$
t_{\text {hop }}=2 t_{C h E}+t_{\text {tuning }}
$$

On the other hand, peak service data rate is also limited by the available TDIL memory at the receiver.

Tables 4.3 and 4.4 provide the data rate overhead for CL and EL considering ATSC 3.0 parameters $6 \mathrm{MHz}$ FFT 16k and GI 1/16. One symbol is considered to be required for tuning, and the values for $D_{Y}$ are 2 or 4 . RF-Muxes of 2 and $4 \mathrm{RF}$ channels are considered. The overhead has been calculated with

\footnotetext{
${ }^{2}$ Cell Interleaver is also considered for the Hybrid TIL output sequence, but not shown in Figure 4.5 for simplicity.
} 
Table 4.3: CL Peak service data rate overheads. 6 MHz FFT 16k GI 1/16

\begin{tabular}{|c|c|c|c|}
\hline$R F-M u x$ & $D_{Y}$ & Overhead & Mbps \\
\hline \multicolumn{4}{|c|}{ QPSK 4/15 (2.5 Mbps) } \\
\hline 4 & 4 & $51 \%$ & 1.23 \\
\hline 4 & 2 & $37 \%$ & 1.57 \\
\hline 2 & 4 & $37 \%$ & 1.57 \\
\hline 2 & 2 & $26 \%$ & 1.85 \\
\hline
\end{tabular}

Table 4.4: EL Peak service data rate overheads. 6 MHz FFT 16k GI 1/16

\begin{tabular}{|c|c|c|c|}
\hline$R F-M u x$ & $D_{Y}$ & Overhead & Mbps \\
\hline \multicolumn{4}{|c|}{$64 Q A M 10 / 15$ (20 Mbps) } \\
\hline 4 & 4 & $51 \%$ & 9.56 \\
\hline 4 & 2 & $37 \%$ & 12.30 \\
\hline 2 & 4 & $37 \%$ & 12.30 \\
\hline 2 & 2 & $26 \%$ & 14.54 \\
\hline
\end{tabular}

respect to the single data rate of $2.5 \mathrm{Mbps}$ and $20 \mathrm{Mbps}$ for the $\mathrm{CL}$ and EL respectively. A maximum $250 \mathrm{~ms}$ frame duration and $2^{19}$ cells of TDIL memory limitation are assumed. The results show that either increasing the number of RF channels in the RF-Mux or decreasing the PP density in the time domain (higher $D_{Y}$ ) makes peak service data rate significantly higher.

\subsubsection{LDM with Channel Bonding}

Figure 4.6 shows the joint LDM and CB transmitter and receiver chains for both LDM layers. It can be observed that the transmitter is composed by one stream partitioner per layer, forming two streams per LDM layer, which are next modulated and combined (CL stream 1 with EL stream 1; CL stream 2 with EL stream 2). In the figure it can be observed that the layers of the LDM signals share the same TIL, PP, and FFT, and are transmitted on both RF channel. The two transmitted LDM signals are received by two independent tuners. The received LDM signals are then demodulated in order to get the CL streams, which are next combined in the CL stream combiner. At the same time, the remodulation and cancellation of the CL streams are carried out in order to obtain the two EL streams. Last, the two EL streams are combined in the EL stream combiner. Notice that the cell exchanger and re-exchanger 

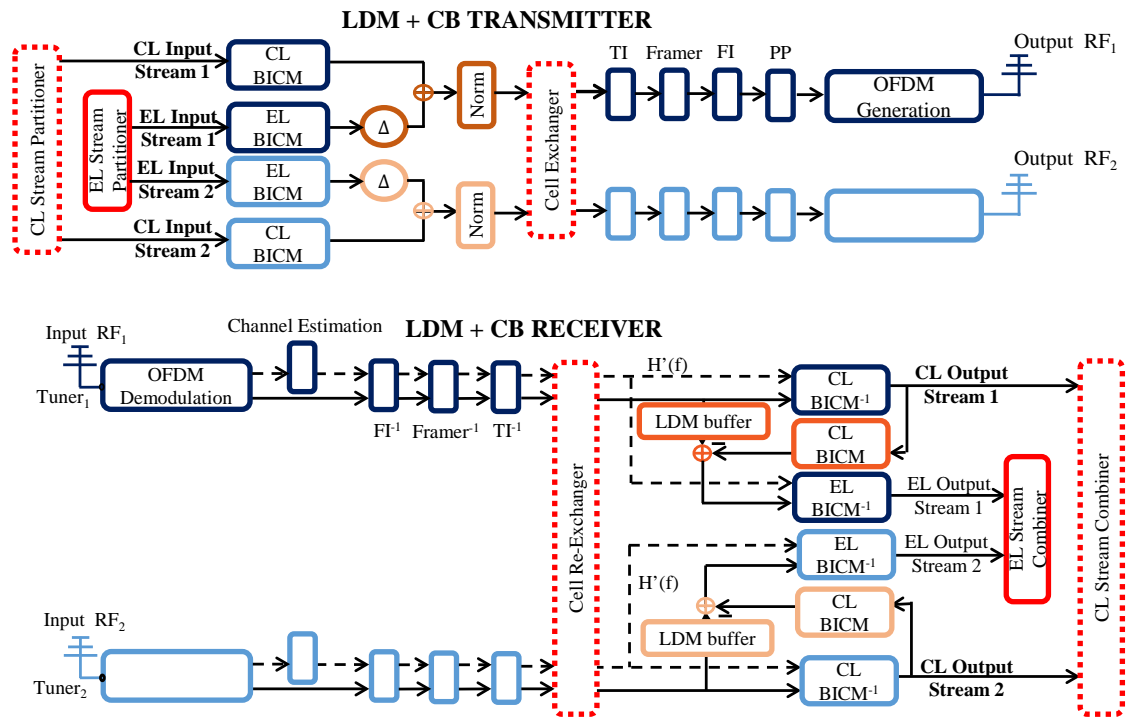

Figure 4.6: $\mathrm{LDM}+\mathrm{CB}$ transmitter and receiver block diagrams. Each LDM layer stream is partitioned into 2 sub-streams, one for each RF channel. Two tuners are needed at the receiver. If CL does not perform CB, CL streams are independent and cell exchanger, cell re-exchanger, CL partitioner, and CL combiner are disabled.

are only allowed when CB is performed to both LDM layers. If there were two independent CL streams, and Plain CB was only performed to the EL, blocks marked with dots would not be implemented (i.e. the CL stream partitioner, CL stream combiner, the cell exchanger, and cell re-exchanger).

The inter-RF frequency interleaving in CB is achieved by means of the cell exchanger. In contrast to TFS, the cell exchanger assigns one half of the cells on RF1 channel and the other half on RF2 channel, independently of the size of the FEC codewords.

\subsection{Methodology and Simulation Setup}

The performance of LDM and MultiRF is evaluated by means of physical layer simulations for mobile and fixed reception with a software simulator validated during the ATSC 3.0 standardization process. The performance of the MultiRF transmission is compared with the transmission over the RF channel with the worst SNR condition, i.e. the one that would be limiting the reception 
of the complete set of services in a classical transmission. Cells of a service are spread in a frame which length is set by the time interleaving duration.

For fixed reception, the effect of transmitting slots of data across different RF channels is emulated taking into account the SNR imbalances between RF channels according to the statistical model presented in [94]. This model accounts for the signal strength variations between UHF channels derived from the transmitter antenna systems and propagation. Notice that only the average values of the imbalances between pairs of frequencies are taken into account for these simulations. According to the model, the worst RF channel coincides with the one with highest frequency.

For mobile reception, the degradation caused by Doppler shift is taken into account. For this purpose, each RF channel is modelled by a different Typical Urban (TU-6) channel with a given number of time realizations. Each TU-6 channel is characterized with the Doppler shift given by

$$
f_{d}(\mathrm{~Hz})=v(\mathrm{~m} / \mathrm{s}) \cdot \frac{f_{c}(\mathrm{~Hz})}{c(\mathrm{~m} / \mathrm{s})}
$$

where $f_{d}$ is the Doppler shift, $v$ is the receiver speed, $f_{c}$ is the carrier frequency of the RF channel, and $c$ is speed of light. It can be observed that $f_{d}$ varies according to the carrier frequency of the RF channel assumed. A correlation factor $(\rho)$ is defined in order to represent the potential time correlation for different signals being broadcast from the same station. According to [97], the correlation is approximately stated between 0.4 and 0.8 in urban environments. The process is made by correlating the phase of the TU- 6 channel time realizations tap-by-tap. To emulate the MultiRF transmission, each FEC codeword is split into $N_{R F}$ data slots which are sequentially filtered by the corresponding TU-6 channel realization of the assigned RF channel (with 4 RF channels, the transmission sequence would be $\left.f_{1}, f_{2}, f_{3}, f_{4}, f_{1}, \ldots\right)$.

The transmission and channel parameters assumed for the simulations are the following:

- Ideal channel estimation is considered.

- $6 \mathrm{MHz}$ channel Bandwidth (BW) is used.

- 16k FFT, 1/16 GI fraction are the waveform parameters shared by both layers.

- 50, 100, and 200 ms TIL duration are considered. 
Table 4.5: RF channel imbalances $(d B)$ respect to the lowest frequency

\begin{tabular}{cccccc}
\hline \hline RF1 & RF2 & RF3 & RF4 & RF5 & RF6 \\
$503 \mathrm{MHz}$ & $533 \mathrm{MHz}$ & $563 \mathrm{MHz}$ & $593 \mathrm{MHz}$ & $623 \mathrm{MHz}$ & $653 \mathrm{MHz}$ \\
\hline 0 & -1.1 & -2.15 & -3.15 & -4.09 & -4.99 \\
\hline \hline
\end{tabular}

- The channel model for mobile reception is TU-6 channel.

- The speeds evaluated on the mobile performance are $3,10,20,30,70$, 100 , and $160 \mathrm{~km} / \mathrm{h}$.

- The correlation factor between RF channels considered on the first study are $\rho=0,0.3,0.5,0.7,0.9,1$. For the rest of the studies, a correlation factor of $\rho=0.7$ is assumed.

- The channel model for fixed reception adopted is Rice (DVB-F1) channel.

- The transmission mode for the mobile service adopted is QPSK 4/15, $(2,5$ Mbps).

- The transmission mode for the fixed service assumed is 64NUQAM 10/15 (19,5 Mbps).

- $\Delta=4 \mathrm{~dB}$ is considered, which distributes the total transmission power according to $70 \%$ for the CL, and $30 \%$ for the EL, approximately.

An intermediate frequency spacing of $30 \mathrm{MHz}$ has been considered. The main results are based on a RF-Mux of $2 \mathrm{RF}$ channels centered at 503 and $533 \mathrm{MHz}$ carrier frequencies. In addition, in order to evaluate the effect of using more than two RF channels, RF-Muxes of 4 and 6 RF channels are also implemented. The additional RF frequencies are centered at 563, 593, 623, and $653 \mathrm{MHz}$. The imbalances between RF channels and the different Doppler shifts for all the speeds under evaluation are presented in Table 4.5 and Table 4.6 respectively. 
Table 4.6: Doppler shift $f_{d}(\mathrm{~Hz})$ per RF channel

\begin{tabular}{ccccccc}
\hline \hline Speed & RF1 & RF2 & RF3 & RF4 & RF5 & RF6 \\
\hline $3 \mathrm{~km} / \mathrm{h}$ & 1.40 & 1.48 & 1.56 & 1.65 & 1.73 & 1.81 \\
\hline $10 \mathrm{~km} / \mathrm{h}$ & 4.66 & 4.93 & 5.21 & 5.50 & 5.77 & 6.05 \\
\hline $20 \mathrm{~km} / \mathrm{h}$ & 9.31 & 9.87 & 10.43 & 10.98 & 11.54 & 12.10 \\
\hline $30 \mathrm{~km} / \mathrm{h}$ & 13.97 & 14.81 & 15.64 & 16.47 & 17.31 & 18.14 \\
\hline $70 \mathrm{~km} / \mathrm{h}$ & 32.60 & 34.55 & 36.49 & 38.44 & 40.38 & 42.32 \\
\hline $100 \mathrm{~km} / \mathrm{h}$ & 46.57 & 49.35 & 52.13 & 54.91 & 57.69 & 60.46 \\
\hline $160 \mathrm{~km} / \mathrm{h}$ & 74.52 & 78.96 & 83.41 & 87.85 & 92.30 & 96.74 \\
\hline \hline
\end{tabular}

\subsection{LDM and MultiRF Channel Performance Evaluation}

This section presents the results of the performance evaluation of the proposed LDM and TFS/CB use cases with inter-RF frequency interleaving.

A first study assesses the EL performance for the different TIL schemes. Next, the results are mainly focused on the performance of the CL in mobile reception, for different time interleaving durations, correlation factors $\rho$, receiver speeds, and number of RF channels. All the results are obtained for a Bit Error Rate (BER) of $10^{-4}$, since there are no important differences for lower values.

\subsubsection{LDM EL and MultiRF performance for fixed reception. Influence of the TIL scheme}

Different TIL schemes available in ATSC 3.0 (hybrid TIL and CI) as well as the block-type TIL of DVB-NGH are used to evaluate the even distribution of cells for the EL of the LDM+TFS system. For reference, performance is also compared to the ideal distribution of cells as well as the performance of the worst RF channel in each RF-Mux. RF-Muxes of 2, 4, and 6 RF channels are considered. The time interleaving duration is set to $100 \mathrm{~ms}$. The SNR of the $\mathrm{RF}$ channels under evaluation is set according to the methodology explained in the previous section.

Figure 4.7 illustrates the performance of the evaluated cases. The result confirms that the ATSC 3.0 hybrid TIL provides the best performance since it fits the ideal TIL scheme case. Regarding the other TIL schemes, it can be 
Table 4.7: EL with TFS performance losses $(d B)$ for the different TIL schemes with respect to ideal TIL

\begin{tabular}{cccc}
\hline \hline RF-Mux & Hybrid TIL & $C I$ & DVB- $N G H$ BI \\
\hline 2 RF channels & 0 & 0.18 & 0.51 \\
\hline 4 RF channels & 0 & 1.08 & 1.24 \\
\hline 6 RF channels & 0 & 1.36 & 1.86 \\
\hline \hline
\end{tabular}

observed that DVB-NGH provides the worst performance with respect to the ideal whereas the CI is in-between. Table 4.7 summarizes the performance loss of the three TIL schemes under evaluation with respect to the ideal performance.

It can be concluded that the ATSC 3.0 hybrid TIL scheme is the optimum for LDM and MultiRF operation. This scheme is assumed for the rest of the simulations in this paper. Assuming this TIL, the potential gain of interRF frequency interleaving for fixed reception comes from the SNR averaging between the different SNR of the RF channels involved in transmission.

\subsubsection{LDM CL and MultiRF performance for mobile reception}

Performance in mobile reception for the CL is evaluated next. Figure 4.8 depicts the MultiRF gain that can be achieved in pedestrian reception $(v=3$ $\mathrm{km} / \mathrm{h}$ ) assuming just different correlation factors between the two RF channels centered at 503 and $533 \mathrm{MHz}$. No SNR imbalances are considered in this result in order to know the impact of the time-correlation between the channels. It can be observed that the higher the correlation between the channel realizations, the lower the MultiRF gain obtained. For totally uncorrelated channels $(\rho=0)$, the MultiRF gains obtained are in the range $3.5-8.7$, depending on the time interleaver duration. On the other hand, if the channels are totally correlated $(\rho=1)$, no MultiRF gain is achieved for the time interleaving durations assumed.

One important aspect to note is that a higher MultiRF gain is reached with a low time interleaving durations, since higher time interleaving durations do not provide better performance for RF-Mux transmission in contrast with single RF transmission. For large interleaving durations the additional gain by inter-RF frequency interleaving is limited. However, there is a significant improvement for lower interleaving durations. 


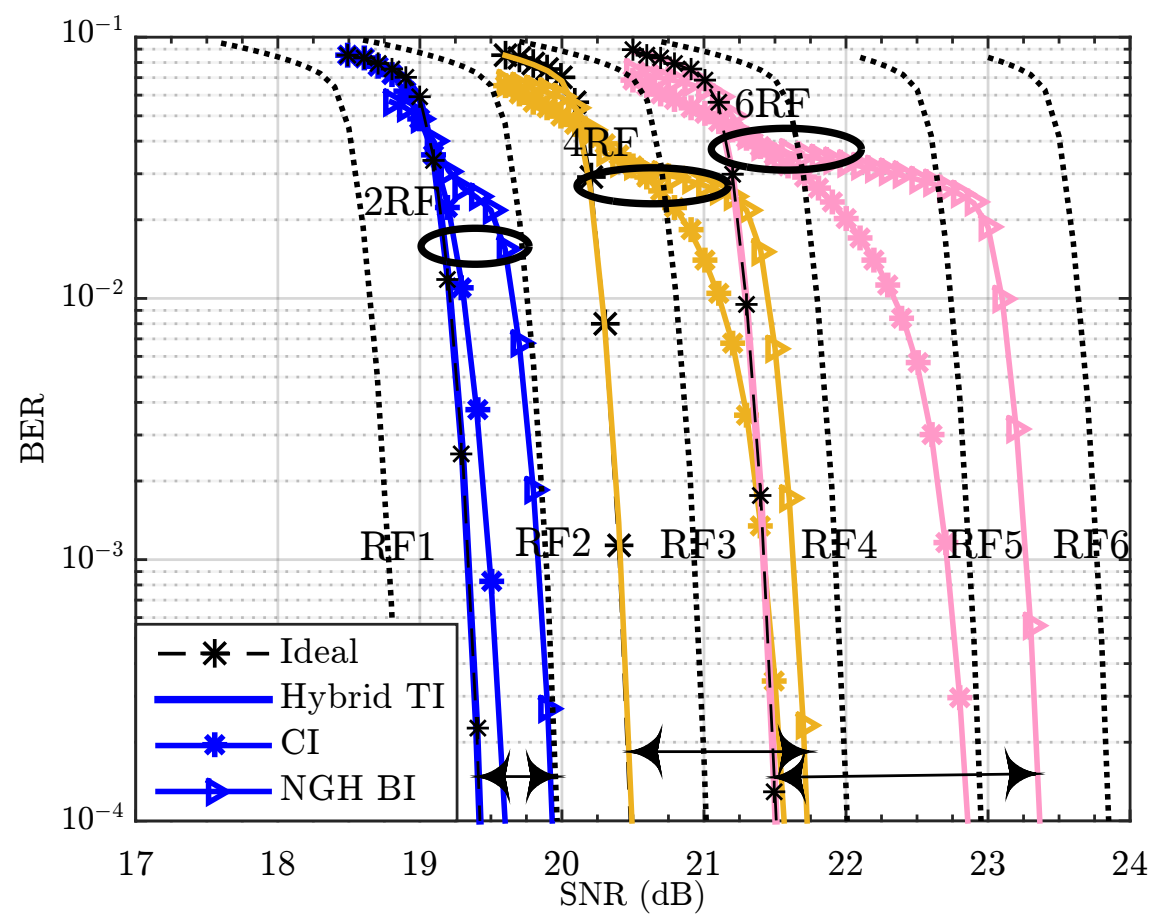

Figure 4.7: Performance of the EL for different TIL schemes for RF-Muxes of 2, 4, and 6 RF channels. 2RF-Mux constituted by RF1 and RF2 (1.1 dB SNR imbalance between best and worst RF channel). 4RF-Mux constituted by RF1, RF2, RF3, and RF4 (3.15 dB SNR imbalance between best and worst RF channel). 6RF-Mux constituted by RF1, RF2, RF3, RF4, RF5, and RF6 (5 dB SNR imbalance between best and worst RF channel).

Assuming $\rho=0.7$, the MultiRF gains for pedestrian reception are 6.5, 4.6, and $2.1 \mathrm{~dB}$ for 50,100 , and $200 \mathrm{~ms}$ respectively.

Fig 4.9 illustrates the MultiRF gains for the CL for different speeds and considering RF channel multiplexes of 2,4 , and $6 \mathrm{RF}$ channels. In this case, the SNR imbalances between RF channels (see Table 4.5) are considered.

For a 2RF-Mux, an imbalance of $1.1 \mathrm{~dB}$ between the two RF channels is assumed. As it was stated above, the lower the time interleaving duration, the higher the achieved gains by inter-RF frequency interleaving. Furthermore, it can be observed that the highest gains are achieved at low speeds. In fact, for speeds higher than $30 \mathrm{~km} / \mathrm{h}$, the gains due to the uncorrelated channel realizations are negligible, regardless the time interleaver duration. The 0.5 


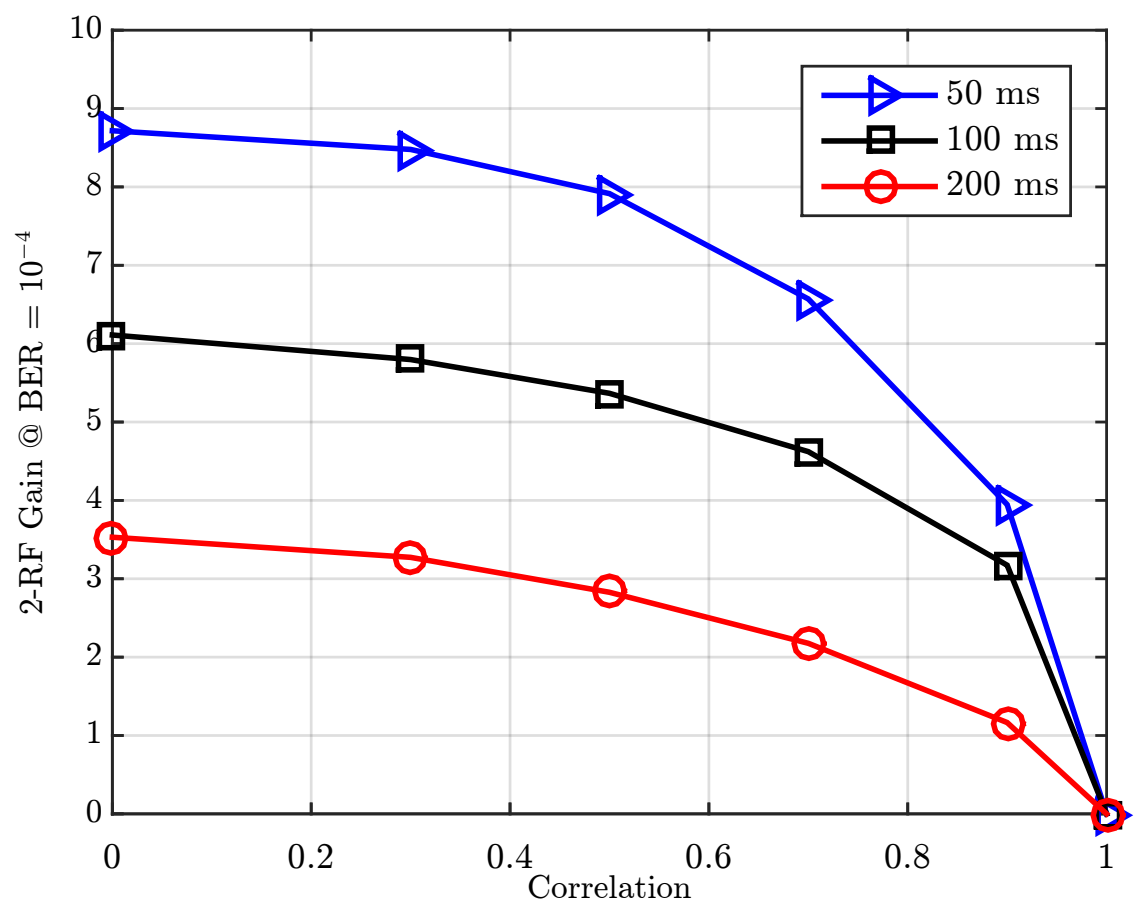

Figure 4.8: $2-\mathrm{RF}$ gain at $\mathrm{BER}=10^{-4}$ for different correlation factor between channel realizations. 50, 100, and $200 \mathrm{~ms}$ interleaving durations.

$\mathrm{dB}$ ground gain of the RF-Mux corresponds to the imbalance between the best and the worst RF channel, and it is independent of the receiver speed.

For a 4RF-Mux, the worst RF channel would have an imbalance of $3.15 \mathrm{~dB}$ with respect to the best $\mathrm{RF}$ channel, and for a $6 \mathrm{RF}-\mathrm{Mux}$ this imbalance ascends to about $5 \mathrm{~dB}$. The reason is based on the higher frequency separation between the worst and the best RF channel in the RF-Mux. For low-speed reception the RF-Mux gains rise up to $12 \mathrm{~dB}$ if a $6 \mathrm{RF}-\mathrm{Mux}$ and a time interleaving duration of $50 \mathrm{~ms}$ is used. In addition, as long as the number of RF channels are increased the gains derived from the uncorrelated channel realizations are extended up to $100 \mathrm{~km} / \mathrm{h}$ for this interleaver duration.

As a summary, Table 4.8 presents the gains for a classical scenario, where a time interleaving duration of $100 \mathrm{~ms}$ is commonly used, with the three RF-Mux compositions for pedestrian reception. It can be seen that the increased gains 


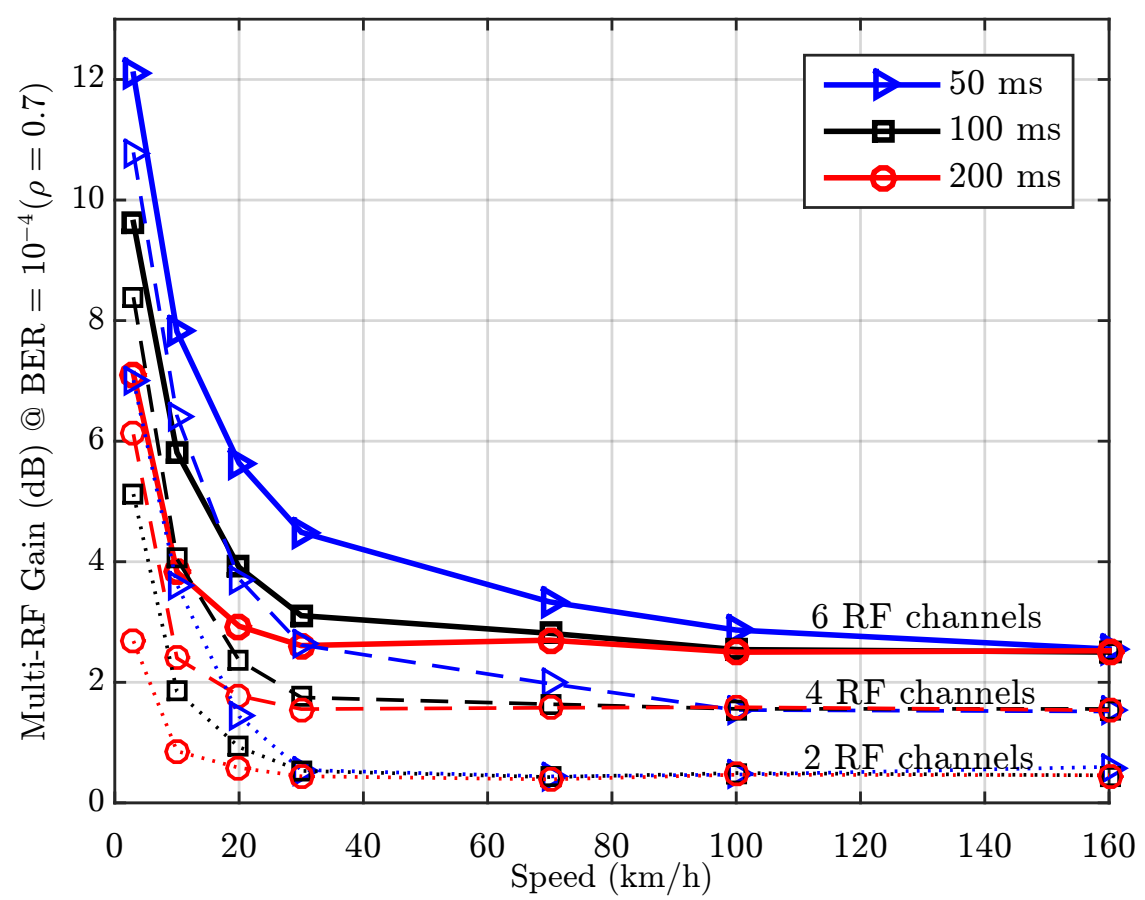

Figure 4.9: Multi-RF gain at $\mathrm{BER}=10^{-4}$ for different speeds. 50,100 , and $200 \mathrm{~ms}$ interleaving durations. Correlation factor of $\rho=0.7$.

of $6 \mathrm{RF}$ channels in comparison with $4(1.3 \mathrm{~dB})$ are less significant than those from the step of 2 to $4 \mathrm{RF}$ channels $(3.2 \mathrm{~dB})$.

\subsection{Conclusions}

This paper investigates the Layered Division Multiplexing (LDM) combination with multiple radio-frequency (MultiRF) channel technologies. Channel Bonding (CB), which only uses 2 RF channels and Time-Frequency Slicing (TFS), that enables the use of up to $6 \mathrm{RF}$ channels, were considered.

There are three main advantages that $\mathrm{CB}$ and/or TFS could offer to LDM: increased peak service data-rate (only with CB), enhanced Statistical Multiplexing (StatMux) thanks to a pool with a large number of services (with $\mathrm{CB}$ and TFS) and/or an increased RF performance due to a higher frequency 
Table 4.8: Multi-RF gains for TIL $=100 \mathrm{~ms} @ 3 \mathrm{~km} / \mathrm{h}$

\begin{tabular}{cccc}
\hline \hline \multirow{2}{*}{$f_{\text {separation }}$} & $2 R F$ & $4 R F$ & $6 R F$ \\
\cline { 2 - 4 } & $30 \mathrm{MHz}$ & $90 \mathrm{MHz}$ & $150 \mathrm{MHz}$ \\
\hline RF Channel Imbalances & $0.5 \mathrm{~dB}$ & $1.5 \mathrm{~dB}$ & $2.5 \mathrm{~dB}$ \\
\hline RF Channel Incorrelation & $4.6 \mathrm{~dB}$ & $6.8 \mathrm{~dB}$ & $7.1 \mathrm{~dB}$ \\
\hline Total Gain $\left(G_{\text {Imb }}+G_{f_{d}}\right)$ & $5.1 \mathrm{~dB}$ & $8.3 \mathrm{~dB}$ & $9.6 \mathrm{~dB}$ \\
\hline \hline
\end{tabular}

diversity by means of an inter-RF frequency interleaving (with CB with SNR averaging and TFS).

The joint LDM and CB leads to two possible use cases. One option is that the two LDM layers perform the SNR averaging CB mode. The other is Plain CB for the EL, which allows a simpler mobile receiver implementation. Regarding joint LDM and TFS, there is only one possible use case. TFS must be applied to both layers since its combination takes place before the TFS scheduling is carried out.

Regarding implementation, the combination of LDM with TFS does not always guarantee the desired frequency diversity for both layers since it depends on the Time Interleaver (TIL) scheme employed which is configured according to the CL transmission mode. It is concluded that the hybrid TIL (constituted by a cell interleaving and a twisted block interleaving) provides the best even distribution of data. In addition, TFS produces an overhead in the peak service data-rate that should be taken into account. It was observed that the overhead increases with the number of RF channels (in the worst case, with 4 $\mathrm{RF}$ channels the overhead could increase up to $51 \%$ ).

According to the performance in mobile and fixed reception, it was shown that high gains are obtained with time-uncorrelated channels for pedestrian reception. The gains range from 2.1 to $6.5 \mathrm{~dB}$ for time interleaving durations from 200 to $50 \mathrm{~ms}$ respectively, with a typical factor of $\rho=0.7$.

Important gains can be exploited from the SNR imbalances between the studied $\mathrm{RF}$ channels. These gains increases with the frequency separation between $\mathrm{RF}$ channels but the gain increase from 4 to $6 \mathrm{RF}$ channels is lower ( $8.3 \mathrm{~dB}$ to 9.6 $\mathrm{dB})$ than that from 2 to $4 \mathrm{RF}$ channels ( $5.1 \mathrm{~dB}$ to $8.3 \mathrm{~dB}$ ). 
Chapter 5

\section{Layered Division Multiplexing with Distributed Multiple-Input Single-Output Schemes}

E. Garro, C. Barjau, D. Gomez-Barquero, J. Kim, S.I. Park, N. Hur, IEEE Trans. on Broadcast., in press 2018. Single Frequency Networks (SFNs) provide an increased spectral efficiency compared to the traditional Multiple Frequency Networks (MFNs). However, some coverage areas in SFN can be affected by destructive interferences. In order to reduce these situations, distributed Multiple-Input Single-Output (MISO) schemes have been adopted in the new Digital Terrestrial Television (DTT) standards, Alamouti in DVB-T2 and Transmit Diversity Code Filter Sets (TDCFS) in ATSC 3.0. On the other hand, Layered Division Multiplexing (LDM), a Non-Orthogonal Multiple Access technology, has been adopted in ATSC 3.0 due to its spectral effciency increase compared to Time or Frequency Division Multiplexing (TDM/FDM). The LDM signal is formed by a power superposition of two independent signals, which are designed for different reception conditions (mobile and fixed-rooftop). The combination of distributed MISO and LDM techniques has not been evaluated yet. In this paper the joint transmission of LDM with distributed MISO is analyzed in terms of complexity and the joint performance is evaluated by means of physical layer simulations. 


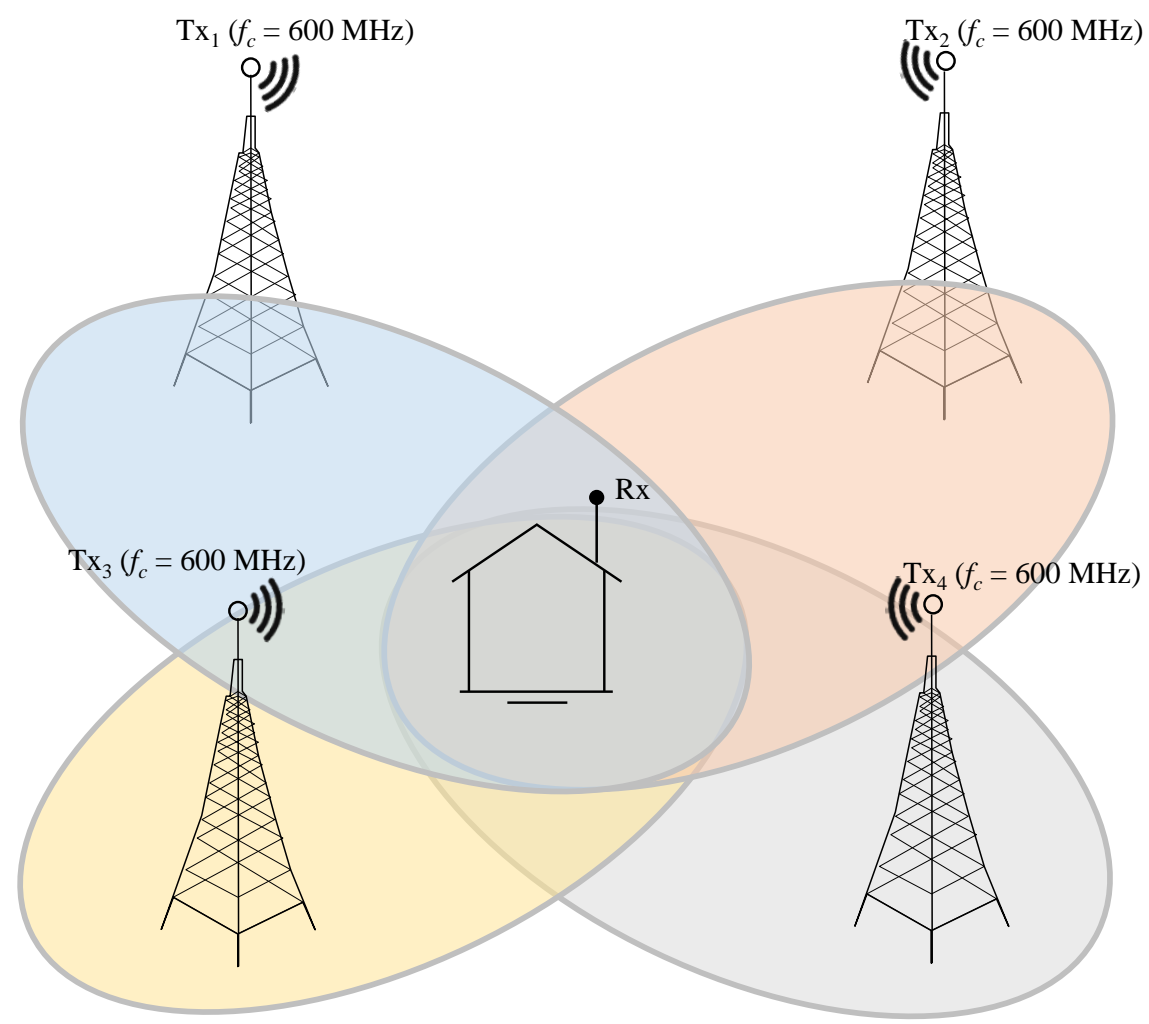

Figure 5.1: SFN constituted by four transmitters that use the same RF frequency $\left(f_{c}=600\right.$ $\mathrm{MHz}$ )

\subsection{Introduction}

A Single Frequency Networks (SFN) is constituted by several time and frequency synchronized transmitters, which send the same signal over the same Radio Frequency (RF) channel. In comparison with the traditional Multiple Frequency Network (MFN), an SFN provides an increased spectral efficiency, as well as a homogeneous distribution of the received signal strength over the coverage area [98], [99]. Figure 5.1 depicts an SFN with four transmitters as an example.

However, some SFN areas can suffer signal degradation. When the same signal from the different SFN transmitters arrives at receivers with similar magnitude and time of arrival but, with different phase, destructive inter- 
ferences may occur. Therefore, severe multipath may come up [69], [100]. In order to limit these destructive interferences, distributed Multiple-Input Single-Output (MISO) schemes have been adopted for the latest Digital Terrestrial Television (DTT) standards. Distributed algorithms alter the SFN, in such a way that the involved transmitters are still time and frequency synchronized, but the transmitted signal is modified. Two distributed MISO algorithms have been adopted in DTT standards, Frequency Pre-Distortion, such as Transmit Diversity Code Filter Sets (TDCFS) in ATSC - Third Generation (ATSC 3.0) [31], and Space Time/Frequency Block Code (ST/FBC), such as Alamouti in DVB - Terrestrial Second Generation (DVB-T2) and Digital Video Broadcasting - Next Generation Handheld (DVB-NGH) [101]. Their benefits have been already analyzed. Nevertheless, the joint transmission of distributed MISO schemes with new transmission techniques is still missing.

Non-Orthogonal Multiplexing (NOM), has emerged as a promising technique for New Radio 5G cellular systems [44]. In contrast to Orthogonal Multiplexing (OM) solutions, such as Time Division Multiplexing (TDM) or Frequency Division Multiplexing (FDM), each multiplexed service in NOM utilizes 100\% of frequency and time resources. Hence, NOM can outperform OM solutions [50]. ATSC 3.0 [26] has become the first terrestrial broadcasting system that implements a NOM solution, known as Layered Division Multiplexing (LDM) [45], [51]. The LDM signal consists of the superposition of two independent layers with different power levels. Each layer, defined as Core Layer (CL) and Enhanced Layer (EL) passes through a different Bit-Interleaved Coded Modulation (BICM) chain. Thus, each layer is designed with different robustness characteristics in order to target mobile reception conditions by the CL, and fixed-rooftop reception conditions by the EL.

Different studies for the joint transmission of LDM with other technologies, such as Multi-Radio Frequency Channel (MultiRF) channel aggregation (Channel Bonding) [32], [102], or Scalable HEVC (SHVC) [90] have been already assessed. However, the joint transmission of distributed MISO schemes with LDM in an SFN has not been evaluated yet. This paper analyzes the implementation aspects at transmitter and receiver sides and it evaluates the joint performance of LDM with the two distributed MISO schemes (TDCFS and Alamouti). On the one hand, although the joint transmission of LDM and TDCFS is currently allowed by ATSC 3.0 standard, the joint performance was not evaluated during the standardization process. On the other hand, the joint transmission of LDM with the well-known Alamouti scheme, adopted in DVB-T2 and DVB-NGH has not been assessed in the literature yet. The rest of the paper is structured as follows: Section 5.2 presents an overview of 
the distributed ATSC 3.0 TDCFS, and DVB-T2 Alamouti schemes. Next, the potential transmitter and receiver implementation aspects of the joint transmission with LDM are analyzed in Section 5.3. Section 5.4 describes the simulation setup followed for the performance evaluation, which is presented in Section 5.5. Last, conclusions are drawn in Section 5.6.

\subsection{MISO Background}

\subsubsection{Frequency pre-distortion}

The frequency pre-distortion approach de-correlates the signals from the different transmitters using a specific linear phase-distortion algorithm. This predistortion has to be unique for each transmitter and has to be different across Orthogonal Frequency-Division Multiplexing (OFDM) subcarriers. This decorrelation enhances the frequency selectivity at receivers so that destructive cancellations are prevented. There are two techniques: the so-called enhanced SFN (eSFN), which was adopted in DVB-NGH [101], and TDCFS, adopted in ATSC 3.0. Compared to eSFN, TDCFS provides a higher decorrelation of the signal in the frequency domain and, thus, an overall better performance [31].

\section{TDCFS in ATSC 3.0}

The linear frequency domain filters are all-pass filters with minimized crosscorrelation under the constraints of the number of transmitters $M \in\{2,3,4\}$ and the time domain span of the filters $L \in\{64,256\}$. Code filter frequency domain pre-distortion function $C_{x}[i]$ is determined using a time domain impulse response vector $h_{x}[n]$ and using a zero-padded Fast Fourier Transform (FFT) of size $N_{\mathrm{FFT}}^{m}$ associated with current subframe $m$. They are introduced in such a way that special signal processing at the receivers is not necessary, since $C_{x}[i]$ are seen by the receivers as a part of the channel. Thus, baseline receivers can also exploit the diversity introduced by these MISO schemes. They are calculated as:

$$
C_{x}[i]=\exp \left[j \arg \left(\sum_{n=0}^{L-1} h_{x}[n] e^{-\frac{j 2 \pi i n}{N_{F F T}}}\right)\right]
$$

where $n$ stands for filter index $n \in\{0, \ldots, L-1\}, h_{x}[n]$ are provided in [17], and $x$ is the transmitter index $x \in\{1, \ldots, M\}$. Subcarrier index $i \in\{0, \ldots, N o C-1\}$, where $N o C$ is the number of active carriers associated to the current subframe. 


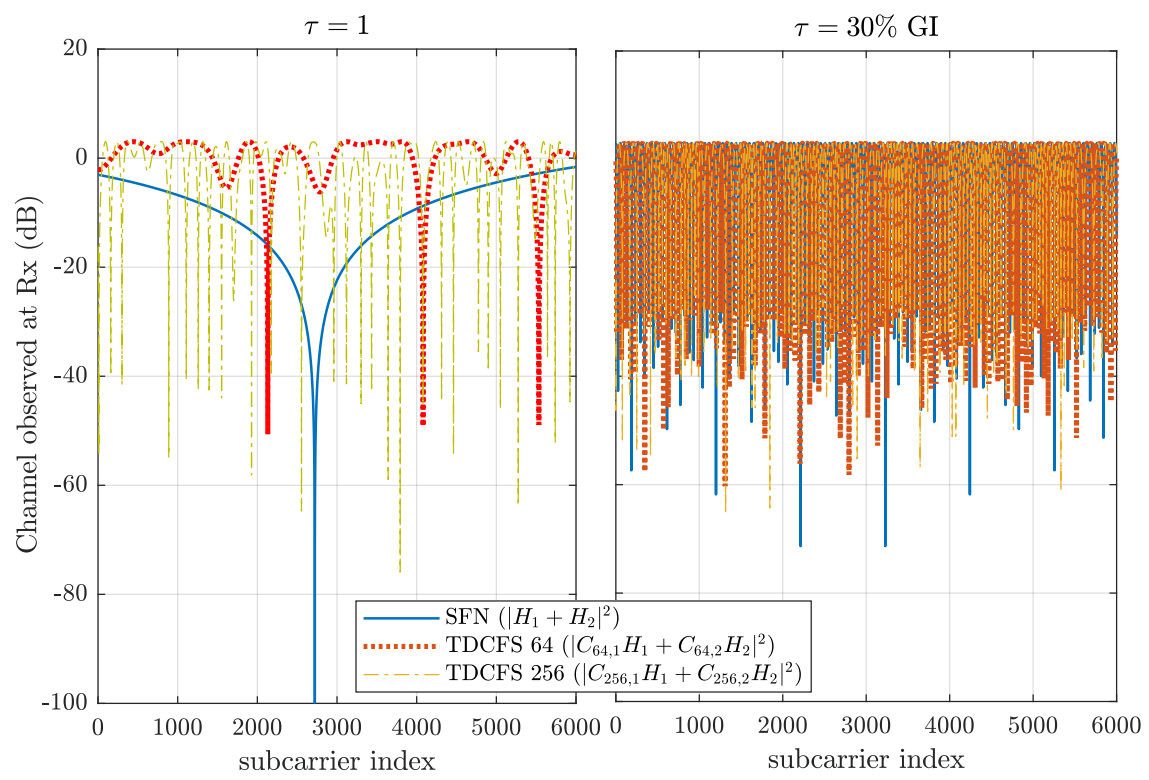

Figure 5.2: Channel frequency response of an SFN with two transmitters and when TDCFS is applied ( $L=64$, and $L=256$ ) (delays $\tau=1$, and 30\%GI samples)

Figure 5.2 shows the Channel Frequency Response (CFR) of an SFN with two transmitters and different delays when TDCFS is applied and when it is not (SFN). As it can be observed, TDCFS pre-distortion modifies the CFR so that no deep fadings occur even at short echo delays. It can also be observed that for medium echo delays (in this case 30\% of GI samples) no performance differences are expected between SFN and TDCFS since the CFRs seem to be equivalent.

\subsubsection{Space-Time/Frequency Block Coding (ST/FBC)}

In the case of ST/FBC, the data stream to be transmitted is encoded in pair of orthogonal blocks, which are distributed among spaced antennas and across time/frequency. Alamouti encoding is the simplest ST/FBC orthogonal design, but at the same time is the only ST/FBC achieving rate-1 [24]. 


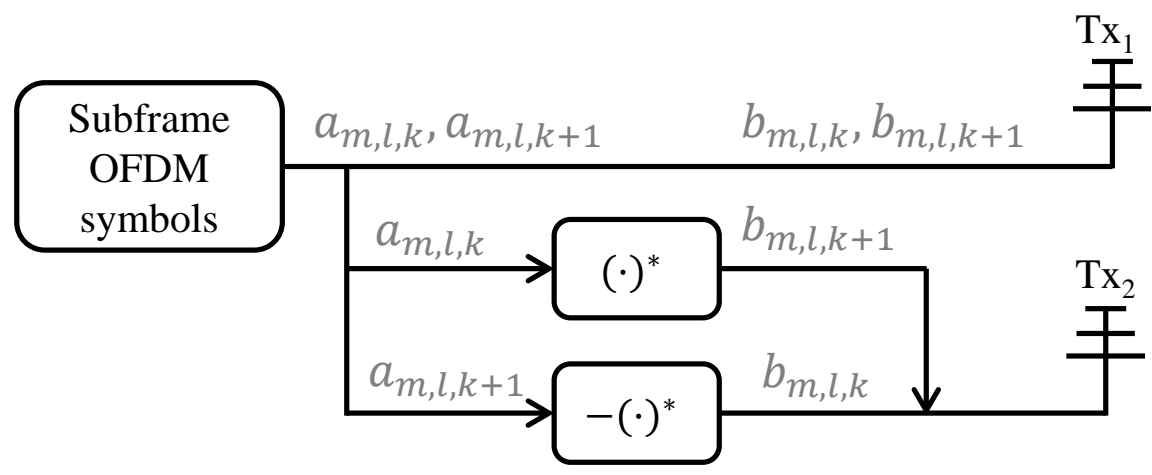

Figure 5.3: MISO Alamouti encoder processing on DTT systems

\section{Alamouti in DVB-T2/DVB-NGH}

An Alamouti encoding variant has been adopted in DVB-T2 and DVB-NGH. In particular, the pair of time indices is replaced by a pair of frequency indices to form an orthogonal Space Frequency Block Code (SFBC). The Alamouti encoding divides the available transmit antennas into two groups. The pair of data carriers $\left\{a_{m, l, k}, a_{m, l, k+1}\right\}$ from group 1 are not modified, while they are complex-conjugated and interleaved from transmit antennas of group 2. The output data carriers $\left\{b_{m, l, k}\left(\mathrm{Tx}_{1}\right), b_{m, l, k}\left(\mathrm{Tx}_{2}\right)\right\}$ for group 1 and 2, respectively are thus encoded according to:

$$
\begin{array}{ll}
b_{m, l, k}\left(\operatorname{Tx}_{1}\right)=a_{m, l, k}, & b_{m, l, k+1}\left(\operatorname{Tx}_{1}\right)=a_{m, l, k+1} \\
b_{m, l, k}\left(\operatorname{Tx}_{2}\right)=-a_{m, l, k+1}^{*}, & b_{m, l, k+1}\left(\operatorname{Tx}_{2}\right)=a_{m, l, k}^{*}
\end{array}
$$

where $l$ denotes the OFDM symbol index, $m$ denotes the subframe index, $k$ denotes the data carrier index $\left(k=\left\{0,2,4,6, \ldots, N_{\text {data }}\right\}\right)$, and $N_{\text {data }}$ is the number of data carriers in an OFDM symbol. The encoding process is illustrated in Figure 5.3.

Alamouti encoding requires not only additional complexity at the transmitter but also at the receiver. Although only a single receiving antenna is needed, the estimation of the CFRs of both transmitted MISO groups is required. Thus, orthogonal Pilot Pattern (PP) between groups should be used. This means that the number of pilots must be doubled for the same channel estimation resolution, so that this pilot overhead should be considered when evaluating the Alamouti gains in terms of spectral efficiency [103]. In addition, MISO Alamouti requires a more complex equalization process for recovering the components from the combined signals. 

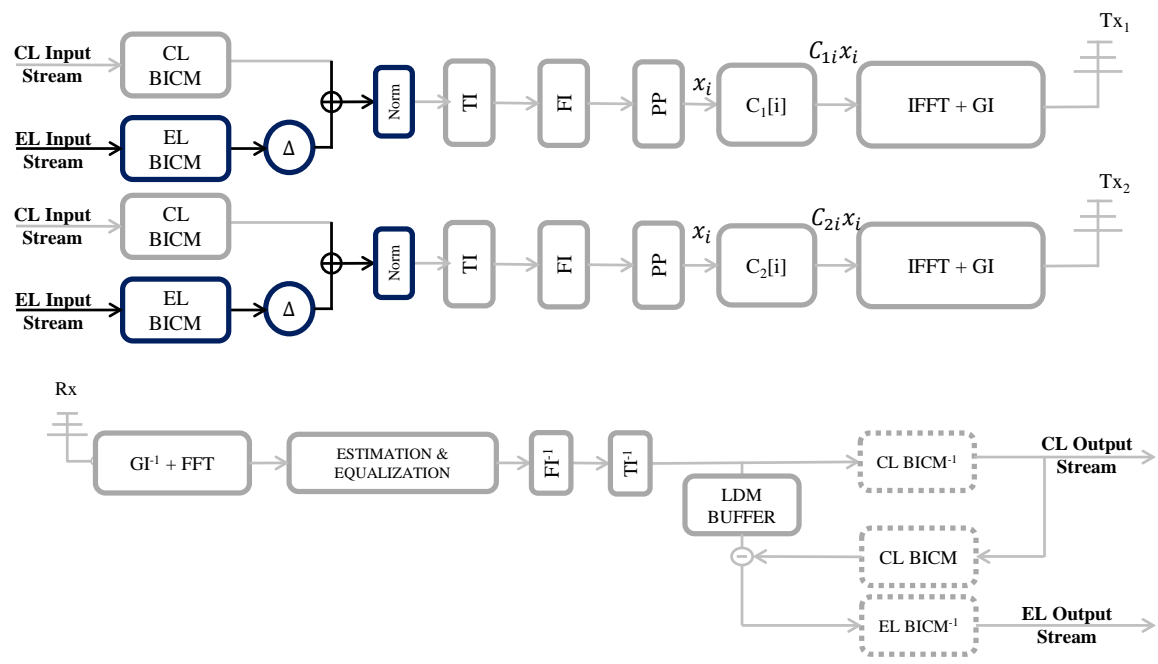

Figure 5.4: Joint MISO TDCFS and LDM block diagram with two transmitters (top) and receiver (bottom)

\subsection{Implementation Aspects of LDM with MISO schemes on ATSC 3.0}

This section evaluates the implementation aspects for ATSC 3.0 transmitters and receivers due to the joint LDM and MISO scheme transmission. Although ATSC 3.0 has only adopted TDCFS as a distributed MISO scheme, Alamouti is also considered in order to provide a more complete study.

\subsubsection{LDM with MISO TDCFS}

Figure 5.4 illustrates two joint LDM and MISO TDCFS transmitters (top) and an ATSC 3.0 LDM receiver (bottom). The two transmitters followed the same block diagram except for the different TDCFS pre-distortion function. Each transmitter applies two BICM chains (one per LDM layer, CL BICM and EL BICM), which are then aggregated with same power allocation controlled by an Injection Level $(\Delta)$. Time Interleaver (TIL) and Frequency Interleaver (FIL) are also equally implemented on both transmitters. Therefore, the same $x_{i}=C L_{i}+E L_{i}$ LDM signal can be observed at this point on both transmitters. Next, a different TDCFS pre-distortion function $C_{x}[i]$ is applied per transmitter, so that whereas $\mathrm{Tx}_{1}$ transmits $C_{1 i} x_{i}, C_{2 i} x_{i}$ is transmitted from $\mathrm{Tx}_{2}$. Finally, inverse-FFT is applied and GI is inserted. From the figure, when 


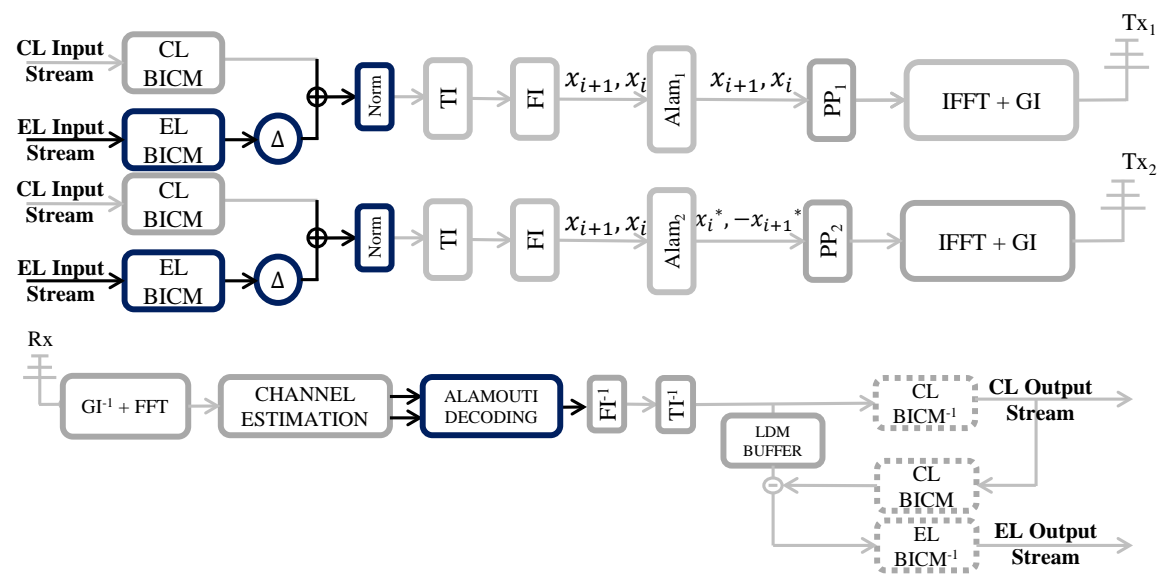

Figure 5.5: Joint MISO Alamouti and LDM block diagram with two transmitters (top) and receiver (bottom)

LDM is jointly used with MISO TDCFS no extra constraints are found, further than those related to each technology by itself. In addition, since TDCFS pre-distortion filters are applied to the combined LDM signal, and assuming same propagation conditions, similar MISO gains in both layers are expected,.

At the receiver, after removing GI and applying the FFT, the complex-valued received signal is modelled as:

$$
y_{i}=\left(h_{1, i} \cdot C_{1}[i]+h_{2, i} \cdot C_{2}[i]\right) \cdot x_{i}+n_{i}
$$

where $\left(h_{1, i} \cdot C_{1}[i]+h_{2, i} \cdot C_{2}[i]\right)$ is assumed by receiver's channel estimator as the CFR and $n_{i}$ is the Additive White Gaussian Noise (AWGN). De-Interleaving processes are next performed, followed by the CL demodulation. If the receiver is expected to retrieve the EL, CL should be first demodulated, remodulated, and cancelled.

\subsubsection{LDM with MISO Alamouti}

In the same way it was shown in previous section, Figure 5.5 illustrates two joint LDM and MISO Alamouti transmitters (top) and the corresponding LDM receiver (bottom).

Similar process as with TDCFS is done until MISO Alamouti encoding is performed in each transmitter. Next, whereas none processing is applied to $x_{i}$ LDM signal in Tx1, Alamouti encoding process complex conjugates and pair- 
wise interleaves $x_{i}$ in Tx2. After Alamouti encoding is processed, orthogonal pilot patterns are applied to each transmitter. Finally, IFFT and GI are applied and inserted similarly to the two transmitters. As the Alamouti encoding is performed over the combined LDM signal $x_{i}=C L_{i}+E L_{i}$, it could be considered that the main MISO Alamouti benefits can also be achieved by both layers.

At the receiver, after removing GI and applying the FFT, the complex-valued received signal is modelled as:

$$
\begin{aligned}
y_{i} & =h_{1, i} \cdot\left(x_{i}\right)-h_{2, i} \cdot\left(x_{i+1}\right)^{*}+n_{i} \\
y_{i+1} & =h_{1, i+1} \cdot\left(x_{i+1}\right)+h_{2, i+1} \cdot\left(x_{i}\right)^{*}+n_{i+1}
\end{aligned}
$$

The received signals $y_{i}$ and $y_{i+1}$ are a combination of the two pair of transmitted cells $x_{i}$ and $x_{i+1}$. Therefore, in order to extract $x_{i}$ and $x_{i+1}$, an Alamouti decoding process is needed after the two CFRs $\left(h_{1}\right.$ and $\left.h_{2}\right)$ have been obtained by the channel estimator. This equalization process is performed as:

$$
\left[\begin{array}{c}
y_{i} \\
y_{i+1}^{*}
\end{array}\right]=\left[\begin{array}{cc}
h_{1, i} & -h_{2, i} \\
h_{2, i+1}^{*} & h_{1, i+1}^{*}
\end{array}\right]\left[\begin{array}{c}
x_{i} \\
x_{i+1}^{*}
\end{array}\right]+\left[\begin{array}{c}
n_{i} \\
n_{i+1}^{*}
\end{array}\right]
$$

After Alamouti decoding, de-interleaving processes are performed before CL is demodulated, which can be cancelled for obtaining the EL. As it can be observed, MISO Alamouti requires of a more complex channel estimation process (two channel estimates are needed) and an extra decoding/equalization at receivers. These blocks are not very complex, but it is evident that the complexity is increased with respect to the previous configuration.

\subsection{Methodology and Simulation Setup}

\subsubsection{Methodology}

The performance of LDM and MISO schemes is evaluated by means of physical layer simulations with a software simulator validated during the ATSC 3.0 standardization process. The performance of the MISO schemes on both layers is compared with the transmission when no pre-processing is applied to the transmitted signal (SFN). In order to provide a fair comparison among the three configurations (SFN, MISO TDCFS and MISO Alamouti), different scenarios have been assumed with realistic channel estimation. 
Table 5.1: BICM and Waveform parameters

\begin{tabular}{ccc}
\hline \hline BICM & \multicolumn{2}{c}{ Waveform } \\
\hline Core Layer & CTI $=1024$ rows & BW $=6 \mathrm{MHz}$ \\
QPSK 4/15 & & \\
\hline Enhanced Layer & Pilot $D_{x}=6$ & Pilot boosting $=4$ \\
64 NUC 10/15 & Pilot $D_{y}=2$ & \\
\hline$\Delta=\{2-6\} \mathrm{dB}$ & FFT $=16 \mathrm{k}$ & GI $=1024$ samples \\
\hline \hline
\end{tabular}

\subsubsection{Transmission Setup}

The common transmitted parameters are introduced in Table 5.1.

\section{Pilot considerations}

On the one hand, a Single-Input Single-Output (SISO) Scattered Pilot (SP) will be used on TDCFS performance evaluation. On the other hand, a MIMO Pilot (MP) must be used with MISO Alamouti scheme. In [104] it was shown that for the FFT 16k - GI 1024 samples, and when a frequency FFT interpolator is used at receivers, $D_{x}=6, D_{y}=2 \mathrm{PP}$ with the maximum pilot boosting power provided the best performance (around $1 \mathrm{~dB}$ gain compared to no boosting). Therefore, ATSC $3.0 S P 6 \_2$ with ATSC 3.0 pilot boosting 4 is assumed for the performance simulations in TDCFS. This means that the pilot carriers are power boosted $4.6 \mathrm{~dB}$ with respect to data carriers' power. Regarding Alamouti pilot configuration, it should be noticed that ATSC 3.0 MP fall on exactly the same positions as for SISO. Thus, MP6_2 with pilot boosting 4 is used in Alamouti results.

Nevertheless, the amplitudes and/or phases of MP may be modified depending on the Multiple-Input Multiple-Output (MIMO) pilot antenna encoding, Walsh-Hadamard (WH) or Null Pilot (NP). In WH, whereas pilots from $\mathrm{Tx}_{1}$ are not modified, pilots from $\mathrm{Tx}_{2}$ are partitioned into two subsets. Phases of the pilots of first subset are not modified, but phases of pilots of second subset are inverted. Thus, the Doppler limit of WH channel estimation is the same as SISO, but the Nyquist limit is halved. On the other hand, in NP encoding, the amplitudes of the scattered pilots of both subsets are modified in both signals transmitted from $\mathrm{Tx}_{1}$ and $\mathrm{Tx}_{2}$. $\mathrm{Tx}_{1}$ alternately transmits scattered pilots with $3 \mathrm{~dB}$ increased transmit power and scattered pilots with null power (zero 
Table 5.2: $\mathrm{MP} D_{x}$ and $D_{y}$

\begin{tabular}{ccc}
\hline \hline \multirow{2}{*}{ SISO } & \multicolumn{2}{c}{ MIMO } \\
\cline { 2 - 3 } & WH encoding & NP encoding \\
\hline$D_{x}$ & $2 D_{x}$ & $D_{x}$ \\
\hline$D_{y}$ & $D_{y}$ & $2 D_{y}$ \\
\hline \hline
\end{tabular}

amplitude). Scattered pilots of $\mathrm{Tx}_{2}$ are transmitted with null power and with $3 \mathrm{~dB}$ gain in reverse order. As a result, for NP, the Doppler limit of channel estimation falls to half compared to SISO, but the Nyquist limit keeps the same. As summary, the equivalent values of $D_{x}$ and $D_{y}$ of each MIMO pilot encoding are summarized in Table 5.2. Both pilot encodings are analyzed for Alamouti in Section 5.5.2.

\section{TDCFS considerations}

Filter length $L=256$ samples is assumed on SFN scenarios with four or less transmitters, as it provides a better performance than $L=64$ samples [31]. A combination of both filter lengths was assumed on scenarios with SFNs constituted by five and eight transmitters.

\subsubsection{SFN scenarios}

The SFN scenarios under evaluation can be grouped in two studies. A first study with just two transmitters but with different delays (0 samples, $1.3 \%$ of GI samples, and $90 \%$ of GI samples) is assumed. Next, scenarios with more than two transmitters are evaluated. The time of arrival of each transmitter in every scenario is summarized in Table 5.3. Regarding echo amplitudes, in order to provide the most challenging SFN conditions, it has been assumed that all echoes arrive at receiver with same magnitude, i.e. $0 \mathrm{~dB}$ echo is always assumed. Figure 5.6 illustrates the Power Delay Profile (PDP) for scenario 7.

When more than two transmitters are considered, different alternatives can be assumed for MISO Alamouti grouping, as well as for MISO TDCFS filtering. Table 5.4 presents the potential grouping alternatives for scenarios 4, and 5, and Table 5.5 for scenario 6. Due to the big amount of grouping alternatives for the performance evaluation of scenario 7, only 0-18-50-90 and 1.3-23-70-95 for group 1 and group 2, respectively, alternative has been evaluated. 
Table 5.3: SFN scenarios under study

\begin{tabular}{ccc}
\hline \hline Scenario & Transmitters & $\tau(\%$ GI) \\
\hline 1 & 2 & 0,0 \\
\hline 2 & 2 & $0,1.3$ \\
\hline 3 & 2 & 0,90 \\
\hline 4 & 3 & $0,18,90$ \\
\hline 5 & 4 & $0,18,70,90$ \\
\hline 6 & 5 & $0,18,23,70,90$ \\
\hline 7 & 8 & $0,1.3,18,23,50,70,90,95$ \\
\hline \hline
\end{tabular}

Table 5.4: Grouping alternatives for scenarios 4 and 5

\begin{tabular}{ccccc}
\hline \hline \multirow{2}{*}{ Grouping } & \multicolumn{2}{c}{ Scenario 4 (3 Tx) } & \multicolumn{2}{c}{ Scenario 5 (4 Tx) } \\
\cline { 2 - 5 } & Group 1 & Group 2 & Group 1 & Group 2 \\
\hline 1 & $0-90$ & 18 & $0-70$ & $18-90$ \\
\hline 2 & $0-18$ & 90 & $0-18$ & $70-90$ \\
\hline 3 & $18-90$ & 0 & $0-90$ & $18-70$ \\
\hline \hline
\end{tabular}

For obtaining the CL performance in mobility conditions, a Typical Urban (TU-6) realization with a Doppler shift $f_{D}=33.3 \mathrm{~Hz}$ is applied to each transmitter path. Regarding EL performance evaluation, uncorrelated realizations with same magnitude but different random phase are applied in order to observe the most destructive interference fixed reception conditions.

\subsubsection{Receiver configuration}

A Least Square (LS) estimation with a moving average time interpolation and a FFT frequency interpolation are considered for the channel estimator. In addition, an Minimum Mean Square Error (MMSE) equalizer is assumed. During the process, it was observed that the performance of the channel estimator mainly depends on the filtering window length of the FFT frequency interpolator. On the one hand, the window length should be sufficiently long for tracing the different echoes of the SFN scenarios. On the other hand, the win- 


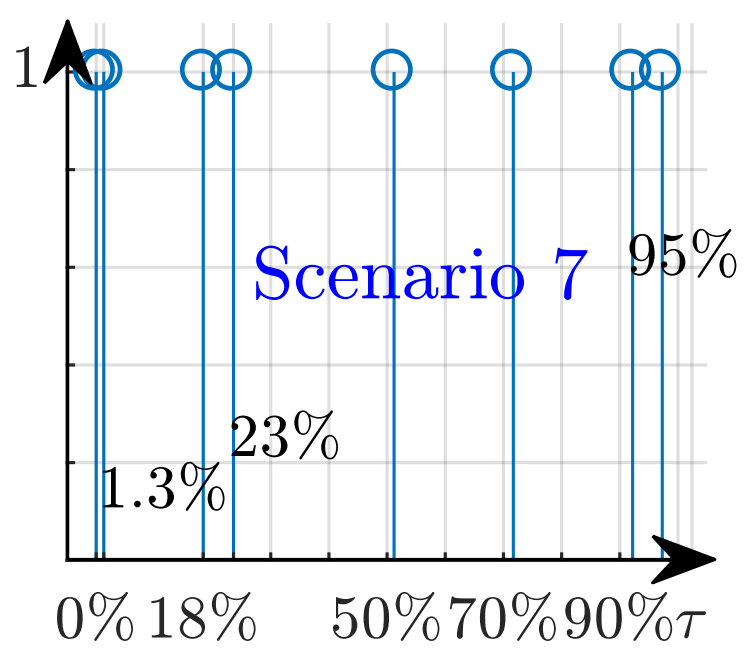

Figure 5.6: PDP of scenario 7. The rest of scenarios are constituted by a subset of this one.

dow length should be as short as possible in order not to increase the frequency domain noise bandwidth.

In order to provide a fair comparison for the whole SFN profiles, three different window lengths were evaluated:

1. $95 \%$ of GI window length for post-echoes and $10 \%$ for pre-echoes.

2. $95 \%$ of GI window length +256 samples of TDCFS filtering for postechoes and $10 \%$ of GI for pre-echoes.

3. $95 \%$ of FFT $/ D_{x}$ window length for post-echoes and $10 \%$ of $\mathrm{FFT} / D_{x}$ for pre-echoes.

For the transmitting configuration under study the window lengths in number of samples are:

1. $\lceil 95 \% \cdot 1024\rceil=973$, and $\lceil 10 \% \cdot 1024\rceil=103$.

2. $\lceil 95 \% \cdot 1024+256\rceil=1229$, and $\lceil 10 \% \cdot 1024\rceil=103$.

3. $\left\lceil 95 \% \cdot \frac{16 \cdot 1024}{6}\right\rceil=2595$, and $\left\lceil 10 \% \cdot \frac{16 \cdot 1024}{6}\right\rceil=274$. 
Table 5.5: Grouping alternatives for scenario 6 (5 Transmitters)

\begin{tabular}{ccc}
\hline \hline Grouping & Group 1 & Group 2 \\
\hline 1 & $0-23-90$ & $18-70$ \\
\hline 2 & $0-18-23$ & $70-90$ \\
\hline 3 & $0-18-70$ & $23-90$ \\
\hline 4 & $0-23-70$ & $18-90$ \\
\hline 5 & $0-70-90$ & $18-23$ \\
\hline 6 & $0-18-90$ & $23-70$ \\
\hline 7 & $18-23-70$ & $0-90$ \\
\hline 8 & $18-23-90$ & $0-70$ \\
\hline 9 & $18-70-90$ & $0-23$ \\
\hline 10 & $23-70-90$ & $0-18$ \\
\hline \hline
\end{tabular}

The performance of the different window lengths was evaluated by comparing the Mean Square Error (MSE) of the channel estimator for the $95 \%$ of GI echo delay. Top part of the Figure 5.7 depicts the channel impulse responses filtered on this scenario with the three configurations and when TDCFS pre-distortion is disabled (left) and enabled (right). Bottom part of Figure 5.7 provides the MSE for the different configurations under consideration.

From top part of the figure it can be seen that the echo is not fully covered by the shortest window length (95\% GI length - purple graph) when TDCFS is applied and when it is not. The omission of the echo is translated in a bad channel estimation, as it is observed in the bottom part. Therefore, this window length is discarded for the performance evaluation of all the proposed SFN profiles. When the other two configurations are compared, it can be observed that $95 \%$ of GI window length +256 samples provides a lower MSE. Hence, taking into account all the potential echo delays, the GI + TDCFS samples length is considered as a valid window filtering length, and it is adopted for the rest of the studies. 

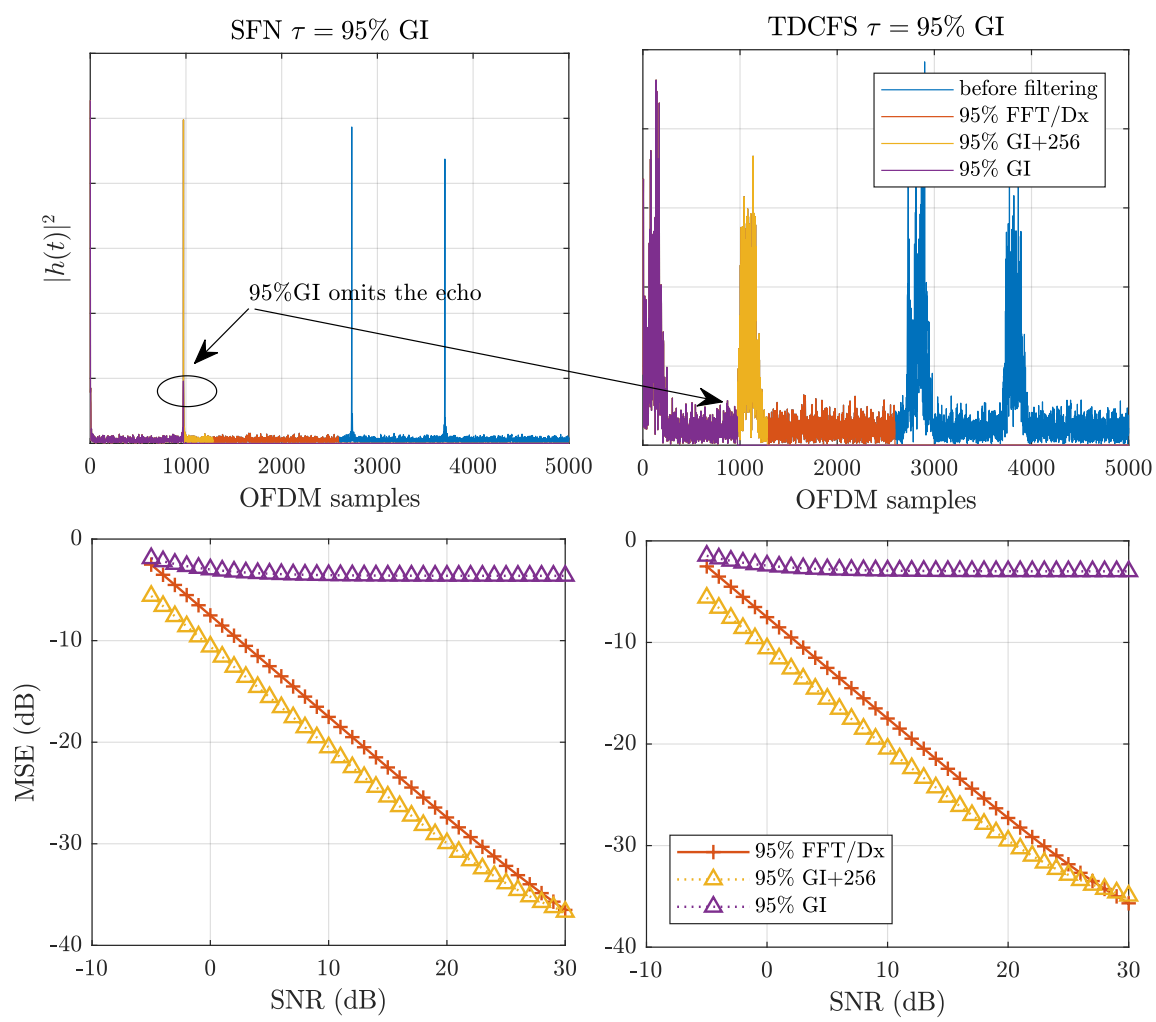

Figure 5.7: Top: SFN (left) and TDCFS (right) channel impulse response in a $0 \mathrm{~dB}$ echo channel with $\tau=95 \%$ GI delay. Bottom: MSE of channel estimator with different FFT frequency interpolators for SFN (left) and TDCFS (right)

\subsection{LDM and distributed MISO Performance Evaluation}

This section studies the potential gains offered by the joint configuration of MISO TDCFS or MISO Alamouti with LDM in comparison with the use of none MISO technique on SFN scenarios. It is divided in three subsections. Subection 5.5.1 evaluates and compares the performance of the CL for all the defined scenarios in previous section. Next, subsection 5.5.2 compares the EL performance for all the scenarios with the three schemes in the same manner as in the CL studies. The performance of both LDM layers is analyzed for an injection level $\Delta=4 \mathrm{~dB}$. The third subsection analyzes the influence of $\Delta$ on MISO Alamouti and MISO TDCFS gains for both LDM layers. 

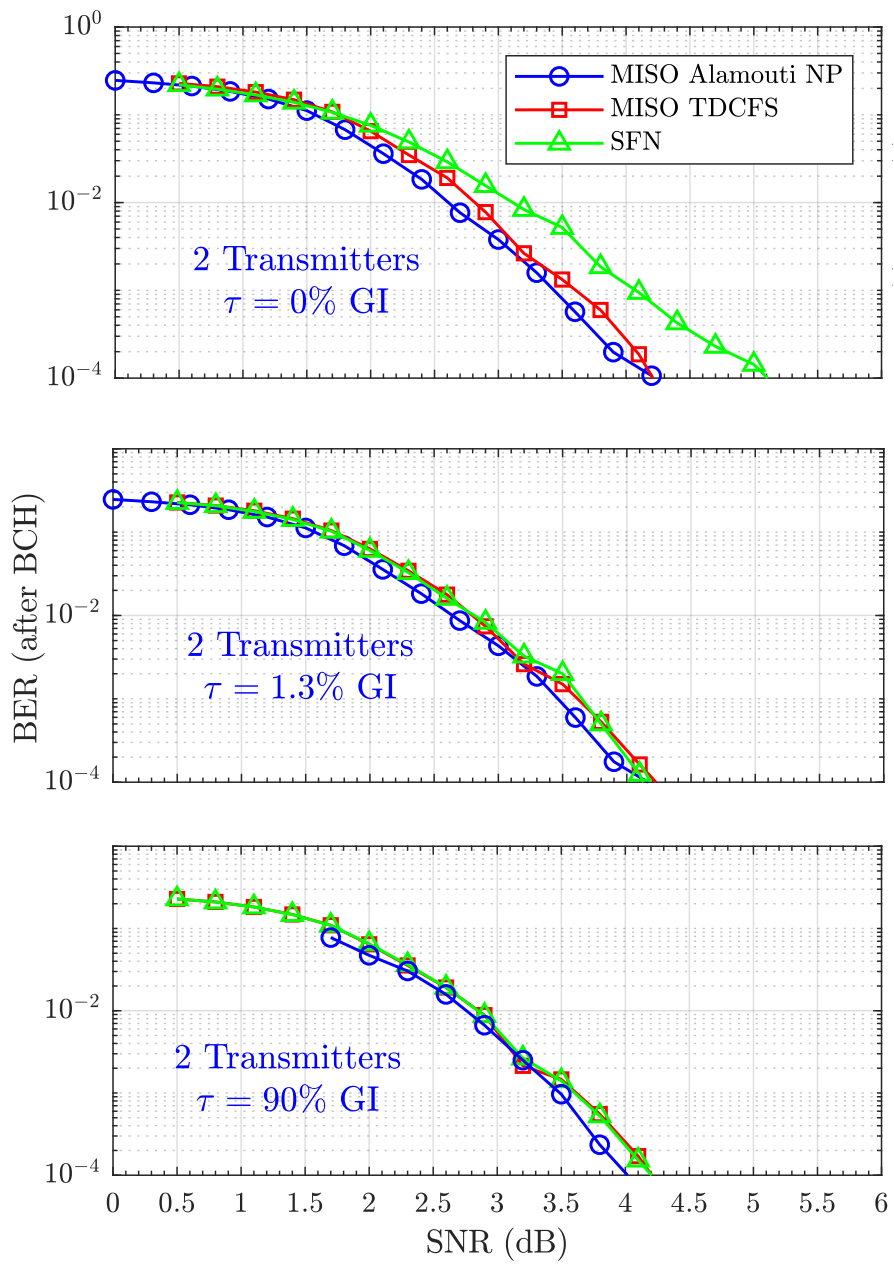

Figure 5.8: Core Layer performance with SFN, MISO TDCFS and MISO Alamouti schemes (with NP encoding) for SFN scenarios with two transmitters $(\tau=0 \%$ (top), $\tau=1.3 \%$ (middle), and $\tau=90 \%$ (bottom) GI samples. LDM injection level $\Delta=4 \mathrm{~dB}$. Uncorrelated TU-6 channel realizations with Doppler shift $f_{D}=33.3 \mathrm{~Hz}$ are applied to each path. 

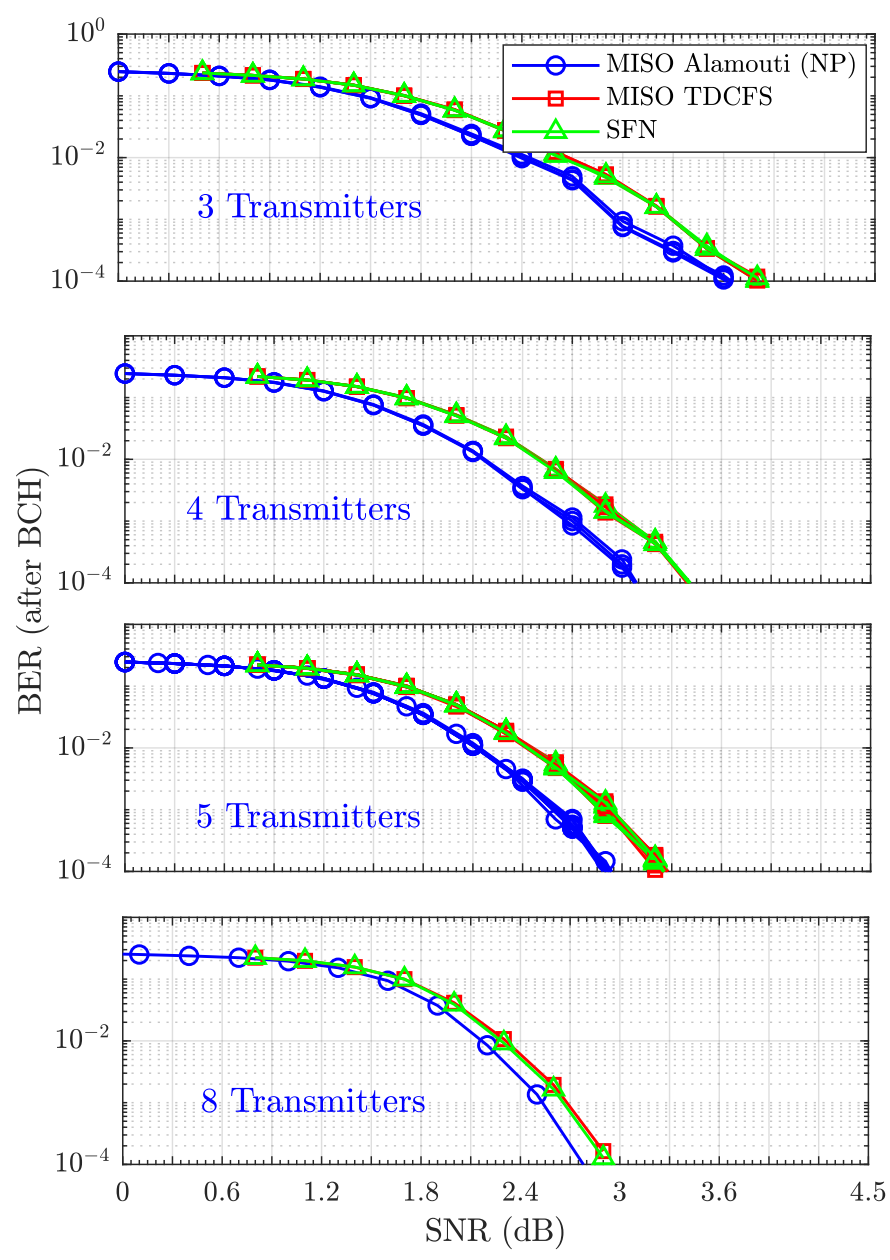

Figure 5.9: Core Layer performance with SFN, MISO TDCFS and MISO Alamouti schemes (with NP encoding) for SFN scenarios with three, four, five and eight transmitters. LDM injection level $\Delta=4 \mathrm{~dB}$. Uncorrelated TU-6 channel realizations with Doppler shift $f_{D}=$ $33.3 \mathrm{~Hz}$ are applied to each path.

\subsubsection{Core Layer Performance}

Scenarios 1, 2, and 3 (2 transmitters)

Figure 5.8 depicts the CL performance for the three scenarios with two transmitters. In general, it can be observed that the performance among the three 
Table 5.6: CL SNR threshold $(\mathrm{dB})$ at $B E R=10^{-4}$ for all the scenarios under evaluation

\begin{tabular}{cccc}
\hline \hline Scenario & MISO Alamouti (NP) & MISO TDCFS & SFN \\
\hline 1 & 4.0 & 4.2 & 5.1 \\
\hline 2 & 4.0 & 4.2 & 4.1 \\
\hline 3 & 3.9 & 4.1 & 4.1 \\
\hline 4 & 3.6 & 3.8 & 3.8 \\
\hline 5 & 3.1 & 3.4 & 3.4 \\
\hline 6 & 2.9 & 3.2 & 3.3 \\
\hline 7 & 2.7 & 3 & 3 \\
\hline \hline
\end{tabular}

schemes is very similar regardless of the echo delay. Only at $\tau=0 \%$ echo delay, $0.8 \mathrm{~dB}$ gains can be observed when MISO Alamouti (with NP encoding) or MISO TDCFS is enabled. As the TU-6 paths form a non-static channel, TIL can obtain enough diversity. Thus, additional MISO spatial diversity gain is not significant. This is known as the diminishing marginal returns of diversity [46].

Scenarios 4, 5, 6, and 7 (more than 2 transmitters)

Figure 5.9 depicts the CL performance for the different grouping alternatives of the scenarios with more than two transmitters. It can be firstly observed that MISO Alamouti (with NP encoding) slightly outperforms SFN and MISO TDCFS by $0.3 \mathrm{~dB}$ in all scenarios. It can also be observed that the performance of the three schemes is not modified whether one grouping alternative is assumed (all curves with the same color/marker are almost overlapped). One last conclusion that can be extracted from Figure 5.9 is that the overall performance of the three schemes increases with the number of transmitters because of the additional diversity.

The Signal-to-Noise Ratio (SNR) thresholds for the three schemes and seven scenarios are summarized in Table 5.6. In summary, it can be concluded that for mobile environments, where the CL is traditionally planned, small gains are obtained by distributed MISO schemes, so that they do not provide a significant performance increase. 

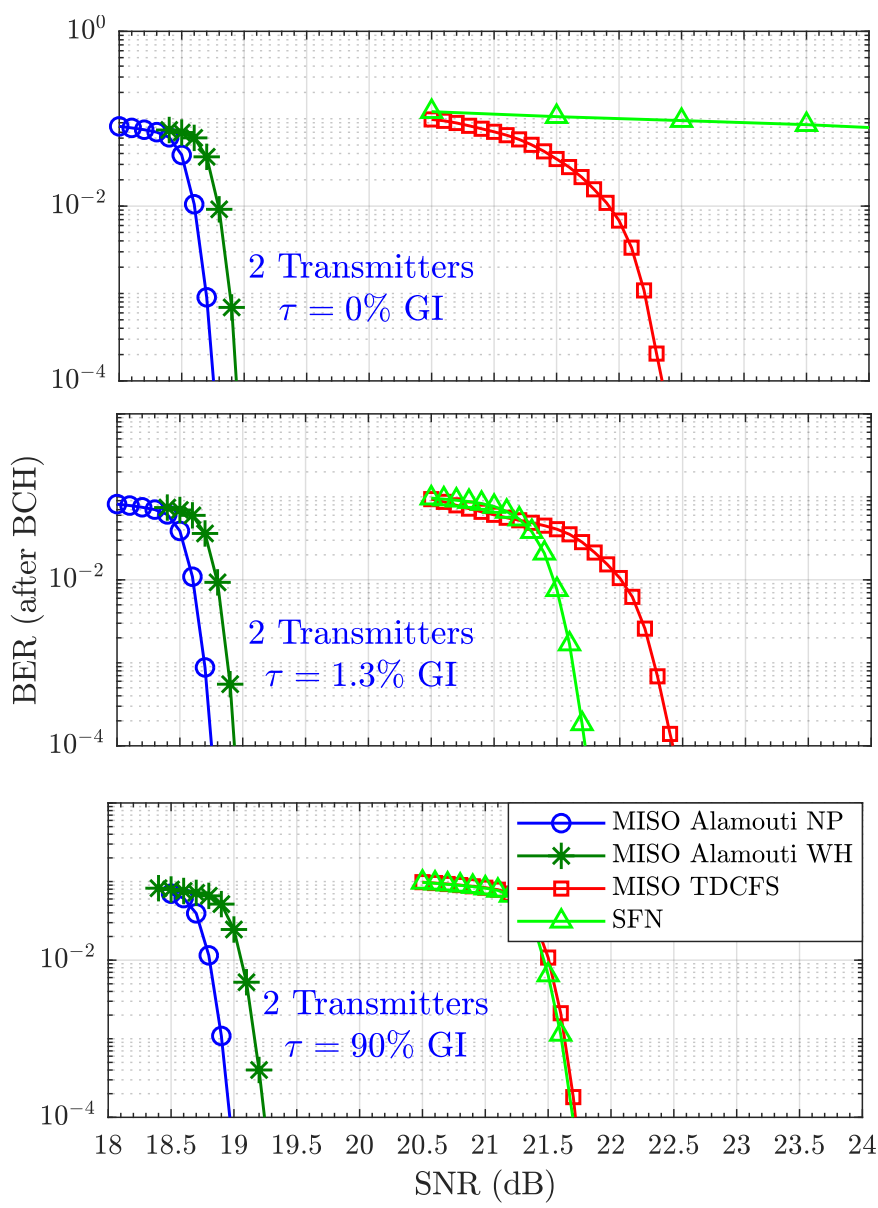

Figure 5.10: Enhanced Layer performance with SFN, MISO TDCFS and MISO Alamouti schemes for SFN scenarios with two transmitters, delayed by $\tau=0 \%$ (top), $\tau=1.3 \%$ (middle), and $\tau=90 \%$ (bottom) GI samples. LDM injection level $\Delta=4 \mathrm{~dB}$. Uncorrelated realizations with same magnitude but different random phase are applied to each channel path. Both MIMO pilot encodings have been considered (Null Pilots - NP, Walsh-Hadamard - WH).

\subsubsection{Enhanced Layer Performance}

For this layer, the two ATSC 3.0 MIMO pilot encodings have been considered on MISO Alamouti scheme. 


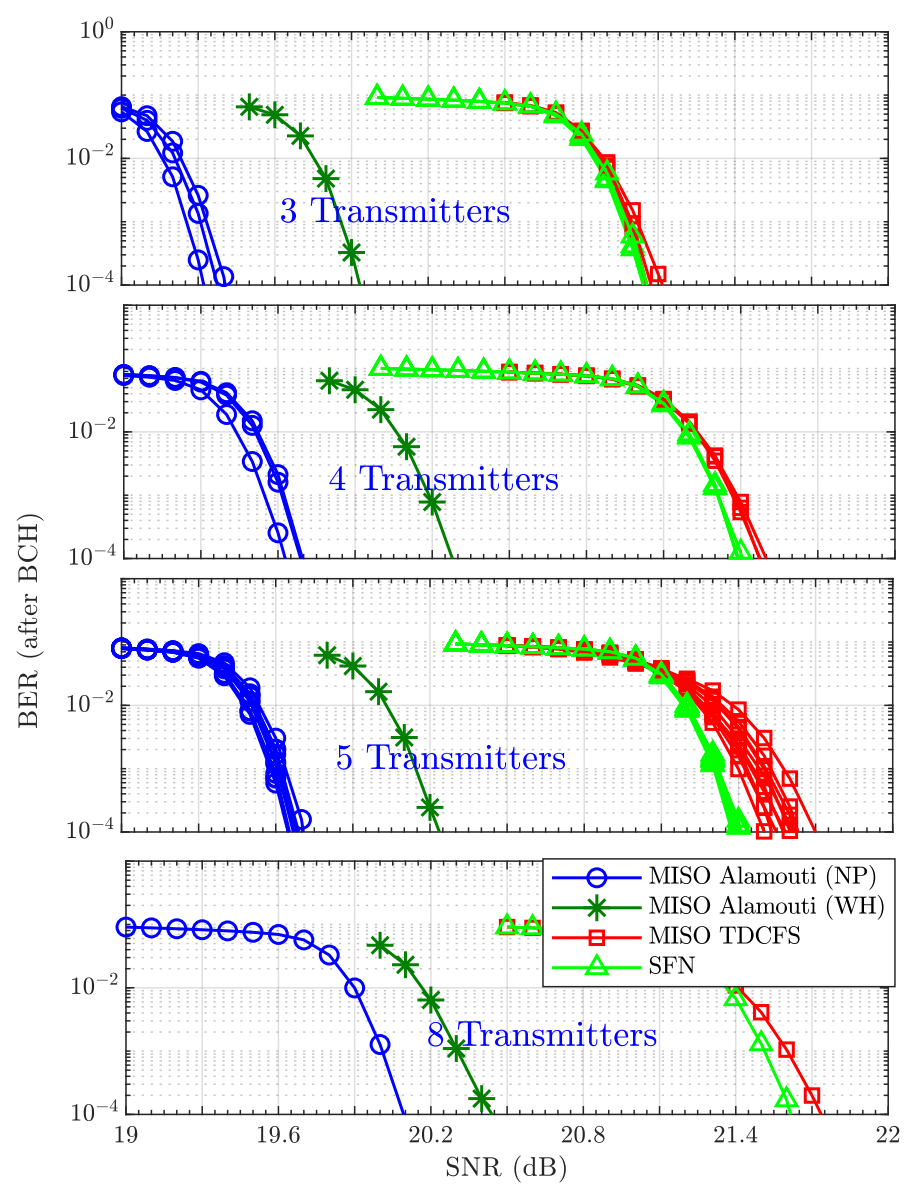

Figure 5.11: Enhanced Layer performance with SFN, MISO TDCFS and MISO Alamouti schemes for SFN scenarios with three, four, five and eight transmitters. LDM injection level $\Delta=4 \mathrm{~dB}$. Uncorrelated realizations with same magnitude but different random phase are applied to each channel path. Both MIMO pilot encodings have been considered (Null Pilots - NP, Walsh-Hadamard - WH).

\section{Scenarios 1, 2, and 3 (2 transmitters)}

Figure 5.10 illustrates the EL performance for the SFN scenarios constituted by two transmitters with the three schemes. It can be seen that for fixed reception conditions, Alamouti with NP encoding now increases the performance up to $3 \mathrm{~dB}$ with respect to SFN. It is explained because TIL is not providing any time diversity, and because of the higher spatial diversity at high CNR 
Table 5.7: EL SNR threshold $(\mathrm{dB})$ at $\mathrm{BER}=10^{-4}$ for all the scenarios under evaluation

\begin{tabular}{cccc}
\hline \hline Scenario & MISO Alamouti (NP) & MISO TDCFS & SFN \\
\hline 1 & 18.8 & 22.4 & NA \\
\hline 2 & 18.8 & 22.4 & 21.7 \\
\hline 3 & 19.0 & 21.7 & 21.7 \\
\hline 4 & 19.4 & 21.1 & 21.1 \\
\hline 5 & 19.7 & 21.5 & 21.4 \\
\hline 6 & 19.7 & 21.6 & 21.5 \\
\hline 7 & 20.1 & 21.8 & 21.7 \\
\hline \hline
\end{tabular}

regions. It can also be noticed that SFN cannot achieve Quasi-Error Free (QEF) conditions for the null echo delay. Nevertheless, this SFN scenario is improved when MISO TDCFS is applied. On the other hand, at short echo delays (such as $1.3 \%$ of GI samples), TDCFS provides a worse performance ($0.3 \mathrm{~dB}$ ) than SFN because of its additional frequency selectivity. If both MIMO pilot encodings are compared, it can be observed that, as it was expected, NP is always outperforming WH. This better performance comes from the $3 \mathrm{~dB}$ boosting at low echo delay SFN profiles, and from the higher echo tolerance at high echo delays.

\section{Scenarios 4, 5, 6, and 7 (more than 2 transmitters)}

The EL performance for the different grouping alternatives of scenarios 47 is presented in Figure 5.11. It can be seen that, similarly than previous scenarios, MISO Alamouti NP outperforms the other schemes. Nevertheless, these gains are reduced to approximately $1.7 \mathrm{~dB}$. The reason comes from the non-optimal Alamouti configuration of only two transmitters involved in the SFN. Again, NP is also outperforming WH. One last conclusion that can be derived from the figure is that the overall performance decreases with the number of transmitters because of the frequency selectivity increase.

The EL SNR thresholds in $\mathrm{dB}$ for the three schemes and seven scenarios are summarized in Table 5.7. In summary, it can be concluded that for fixed environments, where the EL is usually assigned for, MISO Alamouti provides from $1.7 \mathrm{~dB}$ to $3 \mathrm{~dB}$ gains. Hence, the inclusion of this MISO scheme in the ATSC 3.0 standard is recommended. 


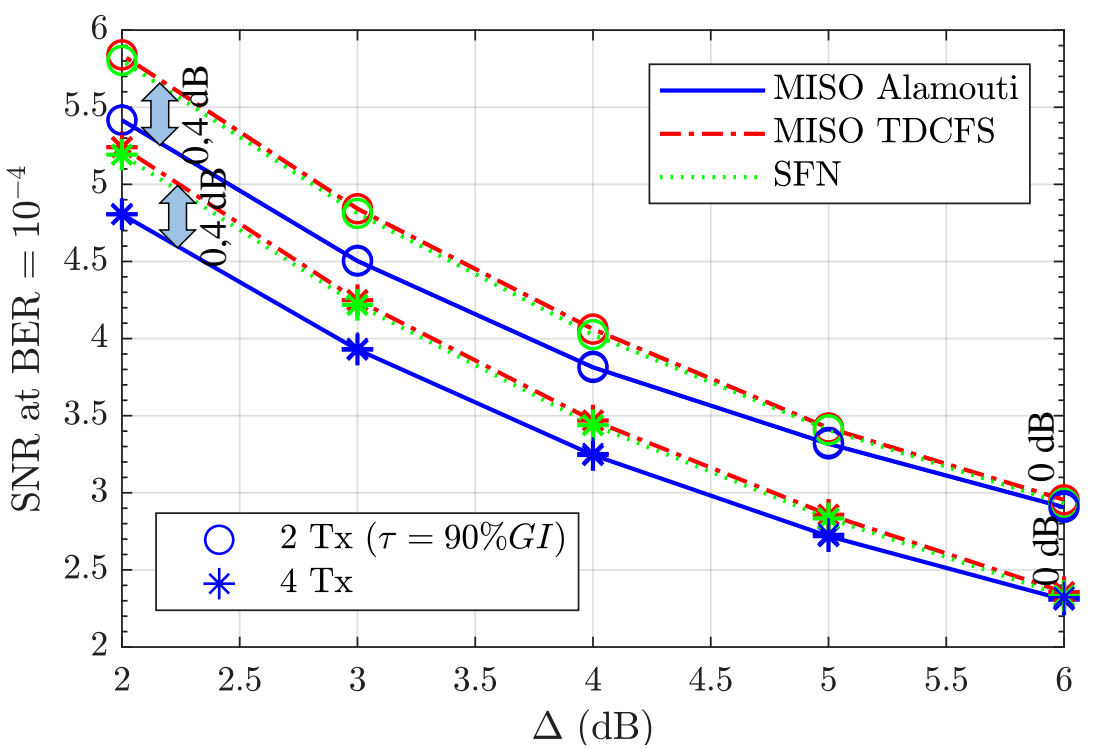

Figure 5.12: Core Layer performance with SFN, MISO TDCFS and MISO Alamouti schemes for $\Delta=\{2-6\} \mathrm{dB}$. MIMO NP encoding is assumed for MISO Alamouti.

\subsubsection{Influence of Injection Level $(\Delta)$}

Previous sections evaluated the performance for the different MISO schemes with a fixed $\Delta=4 \mathrm{~dB}$ value. This section aims at evaluating the influence of $\Delta$ in a wider range. Concretely, $\Delta=\{2-6\}$ dB is evaluated. Scenarios 3 (2 transmitters, $\tau=90 \% \mathrm{GI}$ ) and 5 (4 transmitters) are only considered, since they can be assumed as the two most representative SFN scenarios. Figure 5.12 illustrates the CL performance, while Figure 5.13 depicts the EL performance.

From Figure 5.10, it can be observed that the performance of the three schemes is alike, because of the previously time diversity added by TIL. Nevertheless, small Alamouti gains can be obtained at lower $\Delta$ values, because of the inherent behavior of Alamouti, where gains increase with SNR region. Finally, it can also be seen that the performance increase thanks to the additional time diversity of 4 transmitters scenario remains at $0.6 \mathrm{~dB}$ regardless of $\Delta$. Regarding EL performance, from Figure 5.11, the same conclusions extracted from Section 5.5.2 to $\Delta=4 \mathrm{~dB}$ can be applied to other values: on the one hand, Alamouti always outperforms the other two configurations. Maximum gains of $3 \mathrm{~dB}$ are achieved on two transmitters scenario, whereas these gains are re- 


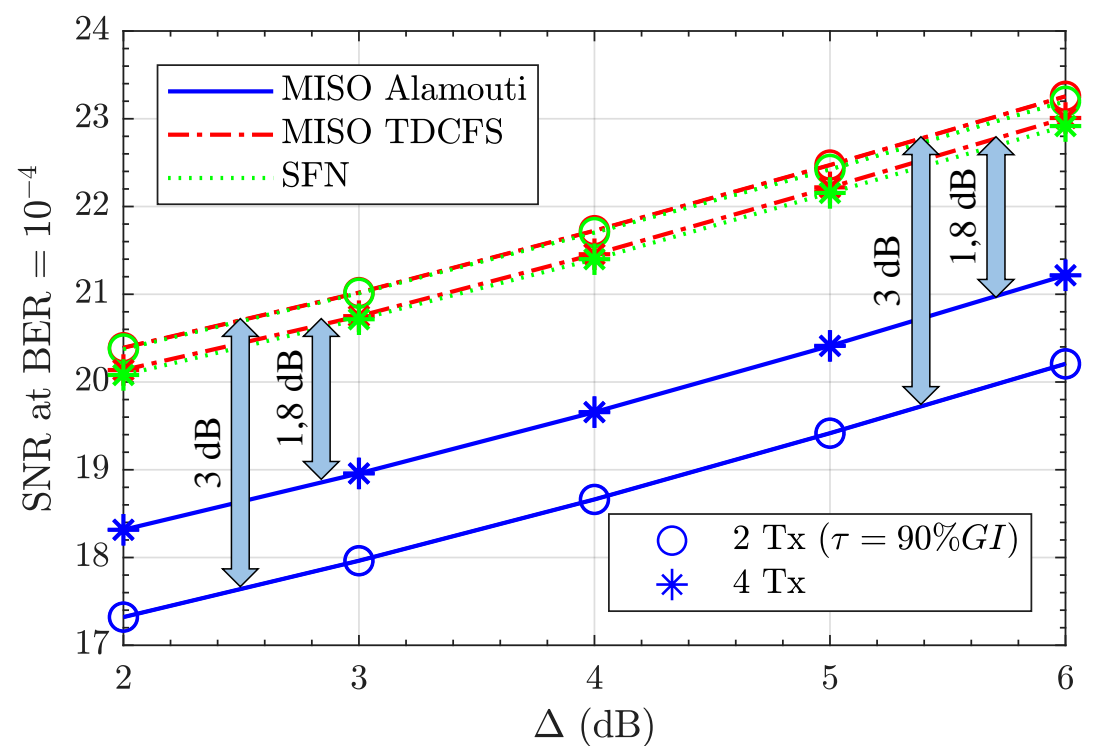

Figure 5.13: Enhanced Layer performance with SFN, MISO TDCFS and MISO Alamouti schemes for $\Delta=\{2-6\} \mathrm{dB}$. MIMO NP encoding is assumed for MISO Alamouti.

duced to $1.7 \mathrm{~dB}$ on four transmitters scenario. Therefore, Alamouti scheme is considered as an optimum configuration for both LDM layers, and in particular for the EL, where up to $3 \mathrm{~dB}$ performance gains can be achieved compared to MISO TDCFS and SFN schemes.

\subsection{Conclusions}

This paper studies the joint transmission of ATSC 3.0 Layered Division Multiplexing (LDM) mode with distributed Multiple-Input Single-Output (MISO) schemes. Two alternatives have been considered, ATSC 3.0 predistortion scheme, namely TDCFS, and DVB-T2 Space Frequency Block Code scheme, also known as MISO Alamouti. Whereas TDCFS de-correlates the signals from the different transmitters to avoid destructive interferences, Alamouti encoding achieves full diversity by sending the same but orthogonal signals between transmitters. This is done in pair of consecutive data carriers. On the one hand, although the joint transmission of LDM and TDCFS is currently allowed by ATSC 3.0, the joint performance was not evaluated during the standardization process. On the other hand, the joint transmission of LDM 
with the MISO Alamouti scheme is also analyzed in order to provide a comparison between the different distributed MISO schemes in the literature.

Regarding the implementation aspects, it was observed that the LDM layers are aggregated before any waveform processing, like MISO schemes. Hence, the combination of LDM with TDCFS does not require extra complexity constraints. Nevertheless, the use of MISO Alamouti requires a slightly more complex channel estimator and equalizer.

For the simulated performance evaluation, seven SFN scenarios with different echo delays $(\tau)$ and number of transmitters were considered. No significant MISO gains were obtained for the CL. The MISO spatial diversity gain is not significant because of the time diversity provided by the prior Time Interleaver (TIL). Regarding the performance of the Enhanced Layer (EL), on the one hand Alamouti gains from $3 \mathrm{~dB}$ to $1.8 \mathrm{~dB}$ were achieved for scenarios with two transmitters and more, respectively. These gains are achieved with the MIMO Null Pilot encoding (MIMO NP), which outperforms the traditionally MIMO Walsh Hadamard encoding (MIMO WH) encoding used in DVB systems. On the other hand, TDCFS gains were only shown at the most challenging scenario, where one echo arrives at the same time and magnitude but with inverse phase.

Overall, since Alamouti gains of up to $3 \mathrm{~dB}$ can be achieved, it is proposed to be included into the next DTT standard, despite it requires of a more complex receiver. 
Chapter 6

\section{Layered Division Multiplexing with Co-Located Multiple-Input Multiple-Output Schemes}

E. Garro, C. Barjau, D. Gomez-Barquero, J. Kim, S.I. Park, N. Hur, submitted to IEEE Trans. on Broadcast. in 2018. The most recent standard for broadcast services, ATSC - Third Generation (ATSC 3.0), has adopted co-located Multi-Antenna schemes and Layered Division Multiplexing (LDM) in order to increase the capacity and reliability compared to former Digital Terrestrial Television (DTT) systems. ATSC 3.0 has adopted both technologies separately, but no combination of them is planned yet. Compared to baseline LDM case, use of several antennas allows for diverse parametrization for each layer. This paper analyzes the potential combination of co-located Multiple-Input Multiple-Output (MIMO) schemes with LDM. A trade-off analysis between complexity constraints and performance benefits is evaluated. 


\subsection{Introduction}

ATSC - Third Generation (ATSC 3.0), the new U.S. Digital Terrestrial Television (DTT) standard [26], has adopted two novel technologies, aim at increasing capacity and robustness of the transmitted services: Multiple-Input Multiple-Output (MIMO) [34] and Layered Division Multiplexing (LDM) [51].

Multiple antenna schemes are based on using two or more antennas on transmitter and/or receiver sides in order to improve the quality and reliability of a transmitted service. Whereas Multiple-Input Single-Output (MISO) and Single-Input Multiple-Output (SIMO) can only improve the reliability of the multipath link by using multiple antennas only at transmitter or receiver side, respectively, MIMO, can also improve the bit-rate of the service as well [35]. These benefits can be achieved without additional channel bandwidth and total transmission power [46]. However, additional complexity at both sides of the transmission link are required. On the one hand, the existing transmitting infrastructures needs to be upgraded with additional transmit antennas, power combiners, etc. On the other hand, more sophisticated signal processing as well as more receiving antennas will be required at receiver side.

Complementary to multiple antenna schemes, LDM, a Non-Orthogonal Multiplexing (NOM) [44], has been also adopted in ATSC 3.0. In LDM, the transmitted signal consists of the superposition of two services with different power levels, controlled by the Injection Level $(\Delta)$. Each service, namely layer, is configured with different robustness and capacity characteristics. The Core Layer (CL), intended for mobile reception, is expected to provide a very robust low bit-rate service. The Enhanced Layer (EL), intended to less demanding fixed roof-top reception conditions, harbors a high bit-rate service. LDM increases spectral efficiency compared to Time Division Multiplexing (TDM) or Frequency Division Multiplexing (FDM), as each type of service uses the full Radio Frequency (RF) bandwidth and transmission time resources [45], [50].

The ATSC 3.0 standard has adopted both technologies separately, but no combination of them is planned yet. Although theoretical studies have been conducted in [105], a more detailed study is required. This paper analyzes the potential combination of co-located MIMO schemes with LDM. A trade-off analysis between complexity constraints and performance benefits emerges. The first combination that may be extracted is the use of MIMO Spatial Multiplexing (SM) for the two LDM layers. It will increase the capacity and reliability of the two services. Nevertheless, the low operational Signal-to-Noise Ratio (SNR) region of CL limits the spatial multiplexing gain [106]. More- 

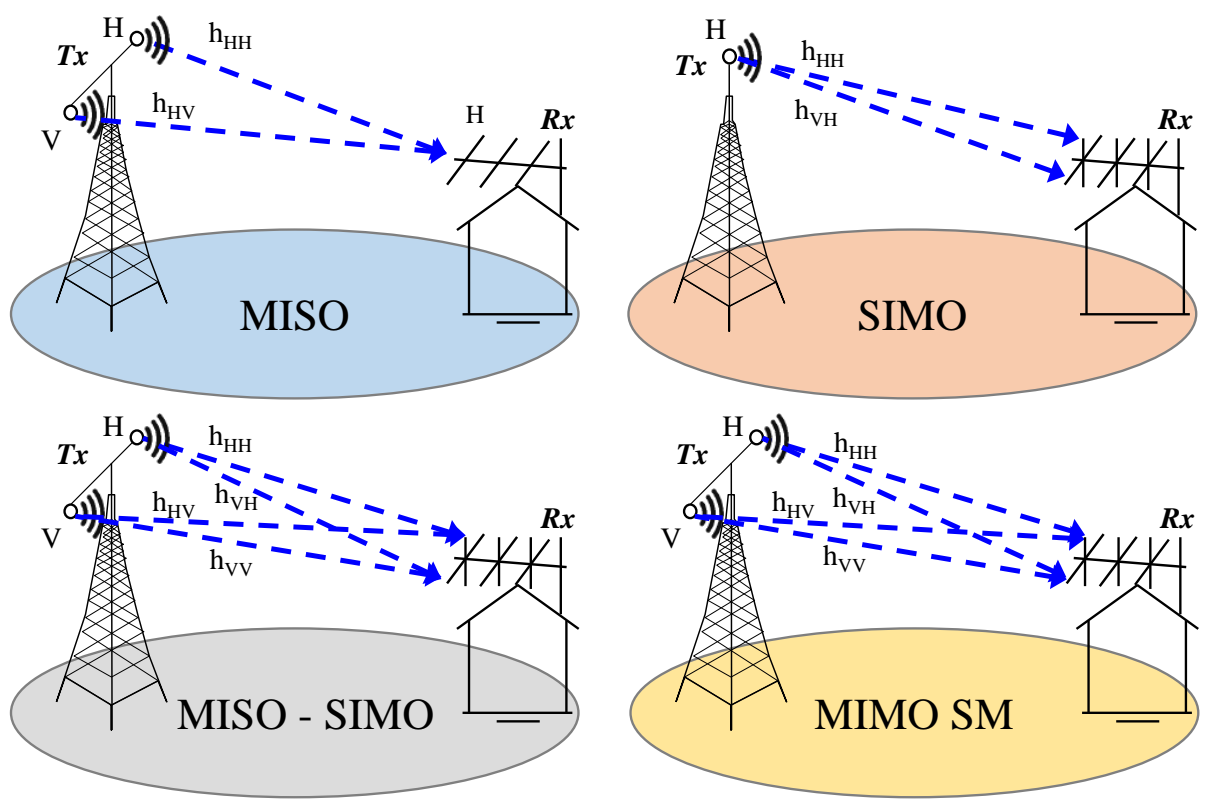

Figure 6.1: Co-Located Multi-Antenna schemes. Top-left: MISO exploits diversity gain. Top-right: SIMO exploits diversity and array gains. Bottom-left: MISO-SIMO exploits diversity and array gains. Bottom-right: MIMO SM exploits diversity, array, and spatial multiplexing gain.

over, it is traditionally oriented for low-complex mobile receivers. Hence, a less complex multi-antenna scheme can be more advisable for the CL.

The rest of the paper is structured as follows: Section 6.2 overviews the multiple antenna schemes employed in terrestrial broadcasting. The potential joint LDM and co-located MIMO use cases are derived in Section 6.3. Section 6.4 analyses the transmitters and receivers implementation aspects for the joint system. Next, Section 6.5 defines the the methodology and the simulation setup used for the performance evaluation results, which are provided in Section 6.6. Finally, conclusions are drawn in Section 6.7.

\subsection{MIMO in Terrestrial Broadcasting}

Depending on the number of transmitting and receiving antennas, co-located MIMO schemes can be divided into four different configurations: 
1. MISO: Only transmitter has two or more antennas. This scheme exploits diversity gain by the use of frequency pre-distortion [31] or SpaceFrequency Block Code (SFBC) [68] techniques.

2. SIMO: Only receiver has two or more antennas. SIMO array gain by the use of combining techniques, such as Maximum Ratio Combining (MRC) [107], can be obtained.

3. MISO-SIMO: It refers to a MIMO system with multiple antennas at both sides. However, transmitter and receiver are not intended to exploit spatial multiplexing gain. In this combination, whereas transmitter exploits MISO diversity gain, the receiver takes advantage of SIMO array gain. This scheme is known as Diversity-MIMO in [103].

4. MIMO SM: In contrast with previous configuration, it uses not only diversity and array gain, but also spatial multiplexing gain.

Figure 6.1 briefly illustrates the four MIMO configurations for terrestrial broadcasting. The two co-located MIMO schemes adopted in ATSC 3.0 are MISO Transmit Diversity Code Filter Sets (TDCFS) and MIMO SM. They are summarized below.

\subsubsection{TDCFS in ATSC 3.0}

TDCFS is a frequency pre-distortion MISO approach. It de-correlates the signals from the different transmitters using a specific linear phase-distortion algorithm. This pre-distortion has to be unique for each transmitter and has to be different across Orthogonal Frequency-Division Multiplexing (OFDM) subcarriers. This de-correlation enhances the frequency selectivity at receivers so that destructive cancellations are prevented. Compared to enhanced SFN (eSFN) [101], the frequency pre-distortion scheme adopted in Digital Video Broadcasting - Next Generation Handheld (DVB-NGH), TDCFS provides a higher decorrelation of the signal in the frequency domain and, thus, an overall better performance. 

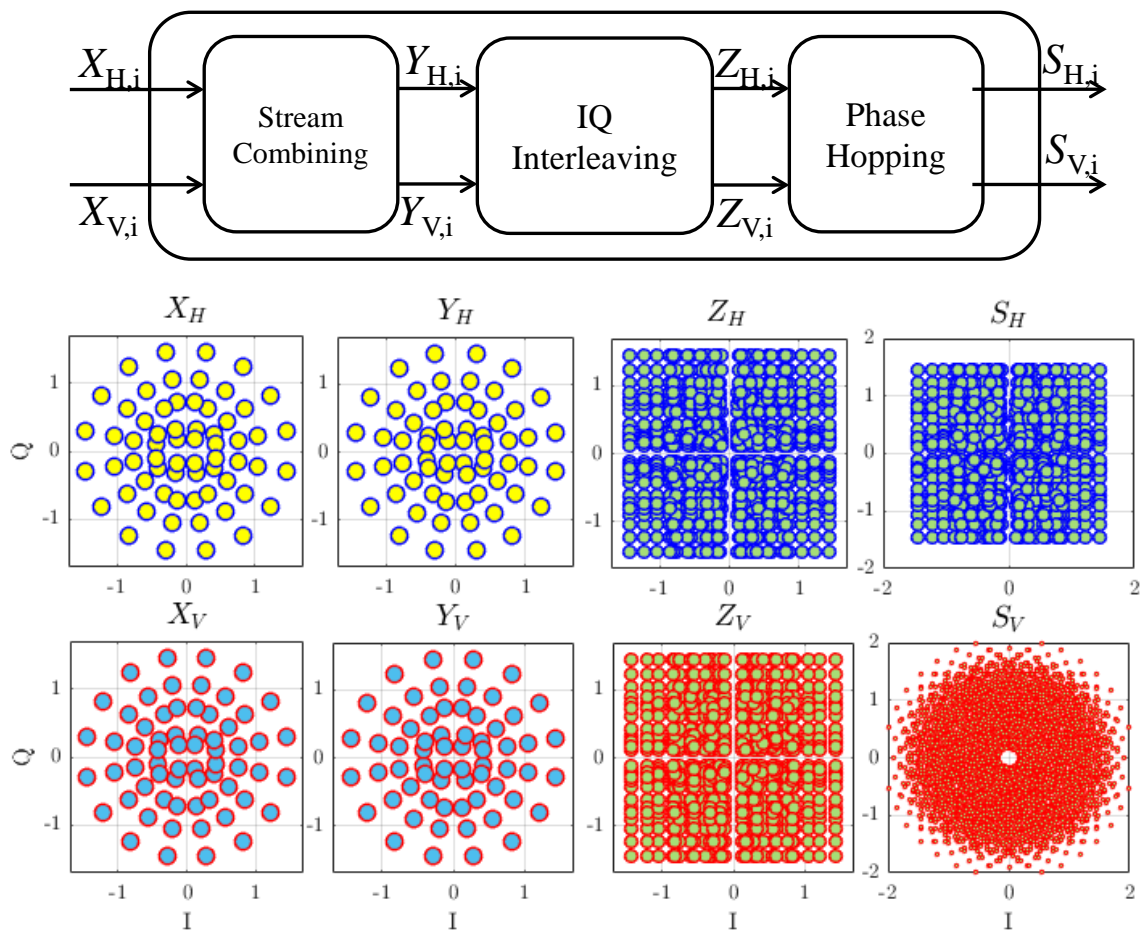

Figure 6.2: ATSC 3.0 MIMO Precoder. Input $\left(X_{H}, X_{V}\right)$ and output $64 \mathrm{NUC}$ constellation after stream combining $\left(Y_{H}, Y_{V}\right)$, IQ polarization interleaving $\left(Z_{H}, Z_{V}\right)$, and phase hopping $\left(S_{H}, S_{V}\right)$

\subsubsection{MIMO in ATSC 3.0}

Since the ATSC 3.0 operational frequency band is Ultra-High Frequency (UHF) band (470-960 MHz), two co-located antennas with cross-polar polarization (i.e. antennas with horizontal and vertical polarizations) are required in order to achieve degree of freedom of 2 , so that it can achieve full spatial multiplexing in Line of Sight (LoS) conditions [108].

Regarding ATSC 3.0 physical layer, same baseline Bit-Interleaved Coded Modulation (BICM) chain [55] as Single-Input Single-Output (SISO) but with an additional MIMO demultiplexer, and a MIMO precoder is used. The MIMO demultiplexer distributes the output bits from the Bit Interleaver (BIL) into two MIMO streams (one per antenna). The MIMO precoder acts on a pair of input constellation symbols within the Forward Error Correction (FEC) block. It is formed by three optional stages: stream combining, IQ polarization in- 
terleaving, and phase hopping, which are described next and illustrated in Figure 6.2.

- Stream Combining: A linear combination of the input constellation symbols based on a rotation angle $\theta$. It depends on the Modulation and Coding Rate (MODCOD) used. If there is no power imbalance between antennas, the optimum rotation angle is $\theta=0^{\circ}$ for all MODCODs.

- IQ Polarization Interleaving: A switching interleaving operation. The output symbol consists of the In-phase (I) component of one input symbol and the Quadrature (Q) component of the other input symbol. It provides an additional diversity gain because each symbol is transmitted in the two cross-polarized antennas.

- Phase Hopping: A phase rotation to the symbols of the second antenna based on the phase rotation angle $\phi(i)=\frac{2 \pi}{9} i, i=0, \ldots, N_{\text {cells }} / 2-1$. It improves the the performance in high correlated channel conditions [109].

The rest of the baseline blocks are doubled, including Time Interleaver (TIL) [30], framing, and Frequency Interleaver (FIL) [82]. Regarding channel estimation, in order to correctly demodulate the two MIMO streams, orthogonal MIMO Pilot (MP) patterns are required.

\section{MIMO Pilots in ATSC 3.0}

In order to properly estimate the four MIMO channel components $\left(h_{H H}, h_{H V}, h_{V H}, h_{V V}\right)$, orthogonal pilot patterns are needed. ATSC 3.0 MP fall on exactly the same positions as Scattered Pilot (SP), i.e. $\mathrm{MP}_{D_{x}, D_{y}}=\mathrm{SP}_{D_{x}, D_{y}}$. Nevertheless, MP amplitudes and/or phases may be modified according to the MIMO pilot encoding used. ATSC 3.0 provides two MIMO pilot encodings, Walsh-Hadamard (WH) and Null Pilot (NP) encoding. Figure 6.3 illustrates the MP6_2 for WH (left) and NP (right) encoding. In WH it can be observed that whereas all pilots from Antenna \#1 are not modified (all green), phases of the scattered pilots from Antenna \#2 are inverted every second pilot bearing carrier (blue). For NP, the amplitudes of the scattered pilots of both antennas are modified. Antenna \#1 transmits alternatively scattered pilots with $3 \mathrm{~dB}$ increased power (red) and with null power (black). Antenna \#2 transmits in the reverse order. These pilot encoding mechanisms thus modify the equivalent $D_{x}$ and $D_{y}$ compared to SISO and will have an impact in the final performance [104], [110]. They equivalent values are summarized in Table 6.1. 

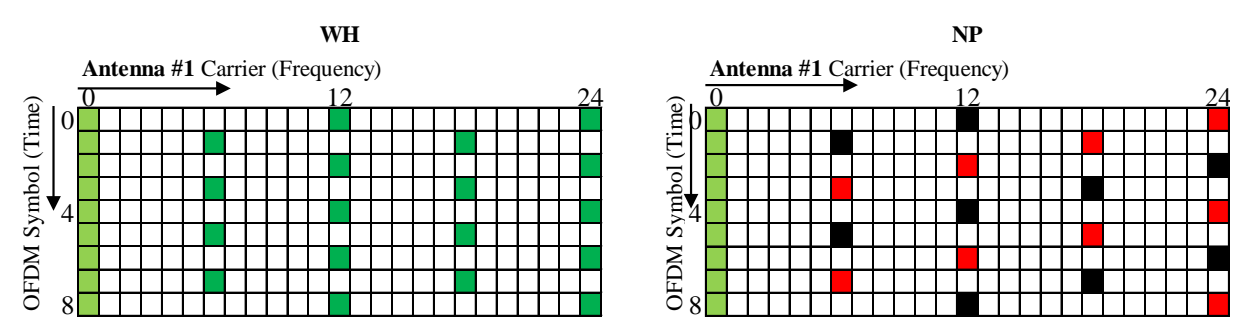

Carrier Number ( Frequency)
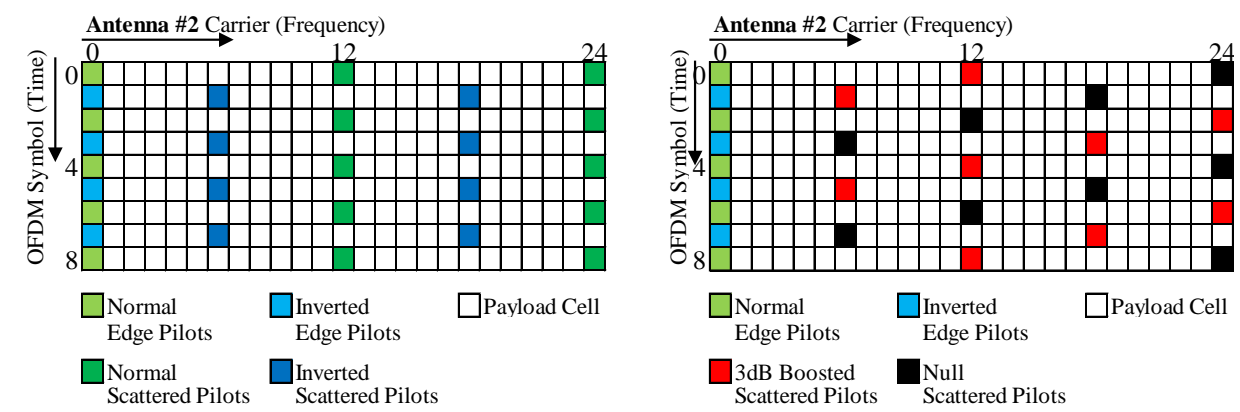

Figure 6.3: MP6_2 for WH (left) and NP (right)

Table 6.1: $\operatorname{MP} D_{x}$ and $D_{y}$

\begin{tabular}{ccc}
\hline \multirow{2}{*}{ SISO } & \multicolumn{2}{c}{ MIMO } \\
\cline { 2 - 3 } & WH encoding & NP encoding \\
\hline$D_{x}$ & $2 D_{x}$ & $D_{x}$ \\
\hline$D_{y}$ & $D_{y}$ & $2 D_{y}$ \\
\hline \hline
\end{tabular}

\subsection{Potential co-located MIMO and LDM use cases}

Taking into account that the bit-rate increase provided by MIMO SM is expected to be fully exploited on the EL, the potential MIMO and LDM use cases (UC) can be grouped into two alternatives, according to the number of antennas of mobile receivers. 


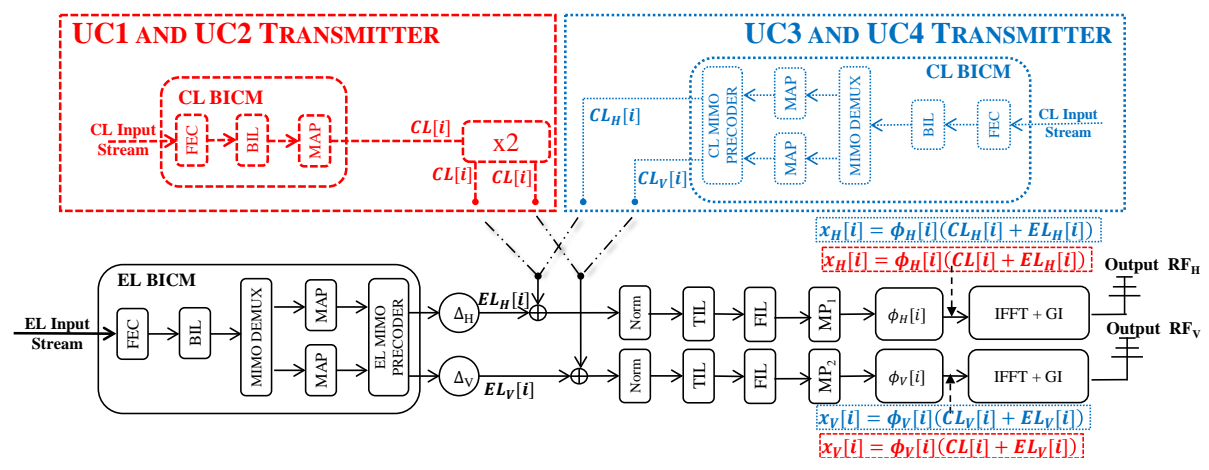

Figure 6.4: Transmitter block diagram for use cases UC1, UC2 (dashed-red), and UC3, UC4 (dotted-blue).

\subsubsection{Mobile receivers with one antenna}

This UC aims at not increasing mobile receivers complexity, but still be able to take advantage of the MISO diversity gain on the CL. On the other hand, fixed receivers can exploit MIMO spatial multiplexing in order to provide higher bit-rates for the EL. This use case can be in turn subdivided into two options depending on the application of TDCFS filtering (UC1 when TDCFS is disabled and UC2 when it is enabled).

\subsubsection{Mobile receivers with two antennas}

This use case is appropriate when there are no restrictions on the mobile receivers' complexity. It exploits not only diversity gain, but also array gain and, even spatial multiplexing gain on the CL. Two options can as well be devised, depending on the activation of the TDCFS filtering (UC3 when TDCFS is disabled, and UC4 when it is enabled).

Figure 6.4 depicts the transmitter block diagram for all the use cases. The main difference among them is the CL BICM chain as well as the TDCFS filtering. For uses cases where mobile receivers have one-antenna (top-left part with red-dashed contour blocks), the CL cell stream is duplicated so that the same information is transmitted in both antenna polarizations. The EL cell stream, which exploits MIMO SM, performs MIMO demultiplexing and MIMO precoding first, and next each EL sub-stream is injected into one of the two CL streams. Hence, two LDM signals are transmitted with different polarizations, $x_{H}[i]=\mathrm{CL}[i]+\mathrm{EL}_{H}[i]$ in horizontal polarization, and $x_{V}[i]=\mathrm{CL}[i]+\mathrm{EL}_{V}[i]$ in vertical polarization. 
Table 6.2: LDM and co-located MIMO schemes use cases

\begin{tabular}{cccccc}
\cline { 2 - 5 } & Use Case & $\begin{array}{c}\text { Mobile } \\
\text { complexity }\end{array}$ & $\begin{array}{c}\text { Div } \\
\text { Gain }\end{array}$ & $\begin{array}{c}\text { Array } \\
\text { Gain }\end{array}$ & $\begin{array}{c}\text { Spatial } \\
\text { Mux Gain }\end{array}$ \\
\hline $\mathbf{1}$ & $\begin{array}{c}\text { MISO Plain CL } \\
\text { MIMO SM EL }\end{array}$ & Low & $\checkmark$ & & \\
\hline $\mathbf{2}$ & $\begin{array}{c}\text { MISO TDCFS CL } \\
\text { MIMO SM EL }\end{array}$ & Low & $\checkmark$ & & \\
\hline $\mathbf{3}$ & $\begin{array}{c}\text { MIMO SM CL } \\
\text { MIMO SM EL }\end{array}$ & Very High & $\checkmark$ & $\checkmark$ & $\checkmark$ \\
\hline $\mathbf{4}$ & $\begin{array}{c}\text { MIMO TDCFS CL } \\
\text { MIMO TDCFS EL }\end{array}$ & Very High & $\checkmark$ & $\checkmark$ & $\checkmark$ \\
\hline \hline
\end{tabular}

For use cases where mobile receivers have two antennas (top-right part with blue-dotted contour blocks), the CL and EL streams pass through independent BICM MIMO SM chains. Thus, both layers are MIMO demultiplexed and MIMO precoded generating $\mathrm{CL}_{H}[i], \mathrm{CL}_{V}[i]$, and $\mathrm{EL}_{H}[i], \mathrm{EL}_{V}[i]$ cell streams. The two LDM signals transmitted with different polarizations are $x_{H}[i]=$ $\mathrm{CL}_{H}[i]+\mathrm{EL}_{H}[i]$ in horizontal polarization, and $x_{V}[i]=\mathrm{CL}_{V}[i]+\mathrm{EL}_{V}[i]$ in vertical polarization. In all the use cases, independent injection level $\left(\Delta_{H}, \Delta_{V}\right)$ between layers can be applied.

Regarding waveform generation, all use cases need of two TIL, two FIL, and orthogonal pilot patterns $\mathrm{MP}_{1}$ and $\mathrm{MP}_{2}$. The difference between UC1 and $\mathrm{UC} 2$, or UC3 and UC4 is the TDCFS application $\left(\phi_{H / V}\right)$. Whereas $\phi_{H / V}[i]=1$ is assumed for $\mathrm{UC} 1$ and $\mathrm{UC} 3, \phi_{H / V}[i]=\exp \left[j \arg \left(\sum_{n=0}^{L-1} h_{H / V}[n] e^{-\frac{j 2 \pi i n}{N_{F F T}}}\right)\right]$ is applied on $\mathrm{UC} 2$ and $\mathrm{UC} 4$, where $L \in\{64,256\}$ refers to time domain span of the TDCFS filters, $h_{H / V}$ are the time domain impulse response vectors provided in [17], and $N_{F F T}$ is the Fast Fourier Transform (FFT) size of current subframe. Finally, Inverse-FFT is applied and Guard Interval (GI) is inserted on each transmitting antenna.

Table 6.2 summarizes the use cases with the potential complexity constraints and performance gains. Next section analyzes the implementation aspects for the four use cases under consideration. 


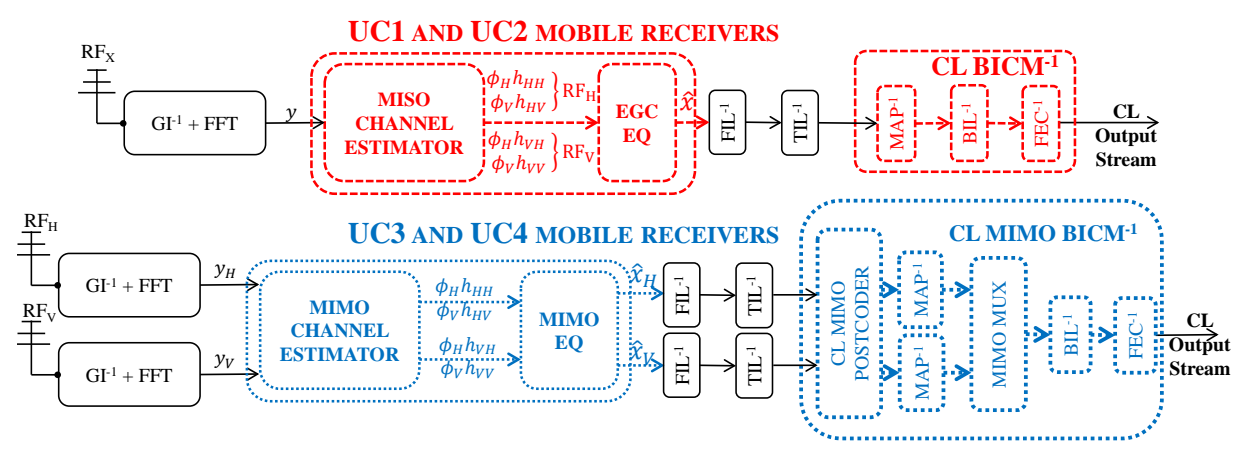

Figure 6.5: Top: Mobile receiver block diagram for the UC1/UC2 (MISO Plain/MISO TDCFS on the CL and MIMO SM on the EL). A more complex channel estimator and equalizer should be used compared to baseline receivers. Bottom: Mobile receiver block diagram for the UC3/UC4 (MIMO SM/MIMO TDCFS on the CL and the EL). A second receiving chain with a more complex MIMO channel estimator, MIMO equalizer and two SISO demappers are needed

\subsection{Implementation Aspects of LDM with co-located MIMO schemes}

This section presents the potential mobile and fixed receivers' block diagrams for all the use cases. Next, it evaluates the latency and memory requirements due to the joint LDM and co-located MIMO scheme transmission.

\subsubsection{Mobile receivers block diagrams}

For the UC1 and UC2, where the CL is transmitted via MISO scheme, the mobile receiver can be implemented with one antenna. Thus, it receives the signal either in horizontal $\left(\mathrm{RF}_{\mathrm{H}}\right)$ or vertical polarization $\left(\mathrm{RF}_{\mathrm{V}}\right)$. The received symbol for these use cases can be expressed as:

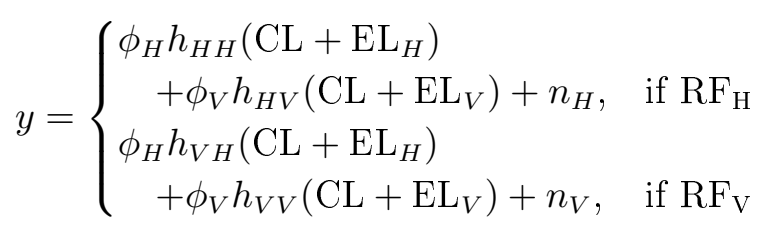

The mobile receiver block diagram, which is expected to only demodulate the CL, can be implemented as top part of Figure 6.5. Since MP are needed for the correct demodulation of the EL, the mobile receiver's channel estimator should take into account the MPs. Thus, it will estimate $\phi_{H} h_{H H}$ and $\phi_{V} h_{H V}$, or 
$\phi_{H} h_{V H}$ and $\phi_{V} h_{V V}$, for horizontal or vertical polarized antennas, respectively. In addition, a slightly more complex Equal Gain Combining (EGC) equalizer should be used in order to combine the two channel estimates [46].

For UC3 and UC4, where the CL is transmitted via MIMO scheme, mobile receivers should be implemented with the two cross-polarized antennas. Thus, the received symbol can be expressed as:

$$
\left\{\begin{array}{l}
y_{H}=\phi_{H} h_{H H}\left(\mathrm{CL}_{H}+\mathrm{EL}_{H}\right) \\
\quad+\phi_{V} h_{H V}\left(\mathrm{CL}_{V}+\mathrm{EL}_{V}\right)+n_{H}, \quad \text { on } \mathrm{RF}_{\mathrm{H}} \\
y_{V}=\phi_{H} h_{V H}\left(\mathrm{CL}_{H}+\mathrm{EL}_{H}\right) \\
\quad+\phi_{V} h_{V V}\left(\mathrm{CL}_{V}+\mathrm{EL}_{V}\right)+n_{V}, \quad \text { on } \mathrm{RF}_{\mathrm{V}}
\end{array}\right.
$$

Bottom part of Figure 6.5 illustrates the mobile receiver block diagram for these use cases. In comparison with $\mathrm{UC} 1 / \mathrm{UC} 2$, mobile receivers should be implemented as MIMO receivers. Hence, a more complex MIMO channel estimator, which will estimate $\phi_{H} h_{H H}$ and $\phi_{V} h_{H V}$ on horizontal polarization antenna and $\phi_{H} h_{V H}$ and $\phi_{V} h_{V V}$ on vertical polarization antenna is needed. Furthermore, a more complex MIMO equalizer, plus two Frequency De-Interleaver (FDIL) and two Time De-Interleaver (TDIL), as well as a CL MIMO post-coder and two CL SISO demappers are needed.

\subsubsection{Fixed receivers block diagrams}

Since EL is going to be transmitted by MIMO SM on the four use cases, fixed receivers will always need two receiving antennas $\left(\mathrm{RF}_{\mathrm{H}}\right.$, and $\left.\mathrm{RF} \mathrm{V}_{\mathrm{V}}\right)$. Hence, the received symbols are expressed as (6.2), for all use cases, keeping in mind, that $\mathrm{CL}_{\mathrm{H}}=\mathrm{CL}_{\mathrm{V}}=\mathrm{CL}$ for $\mathrm{UC} 1 / \mathrm{UC} 2$.

The corresponding block diagrams are depicted in Figure 6.6. Compared to mobile receivers implementation, fixed receivers need to perform the LDM cancellation process in order to obtain the EL. Thus, in addition to the previous blocks, two LDM buffers are required for retrieving $\mathrm{EL}_{\mathrm{H}}$ and $\mathrm{EL}_{\mathrm{V}}$. Moreover, a second $\mathrm{BICM}^{-1}$ chain for the EL is needed. The difference between fixed receivers implementation is observed at the CL demodulation and remodulation. For UC1/UC2, baseline CL BICM and CL BICM ${ }^{-1}$ chains are used, while UC3/UC4, a CL MIMO BICM ${ }^{-1}$ chain, which includes a CL MIMO postcoder, two SISO demappers and a CL MIMO multiplexing, and a CL MIMO BICM chain for its reconstruction are needed. 


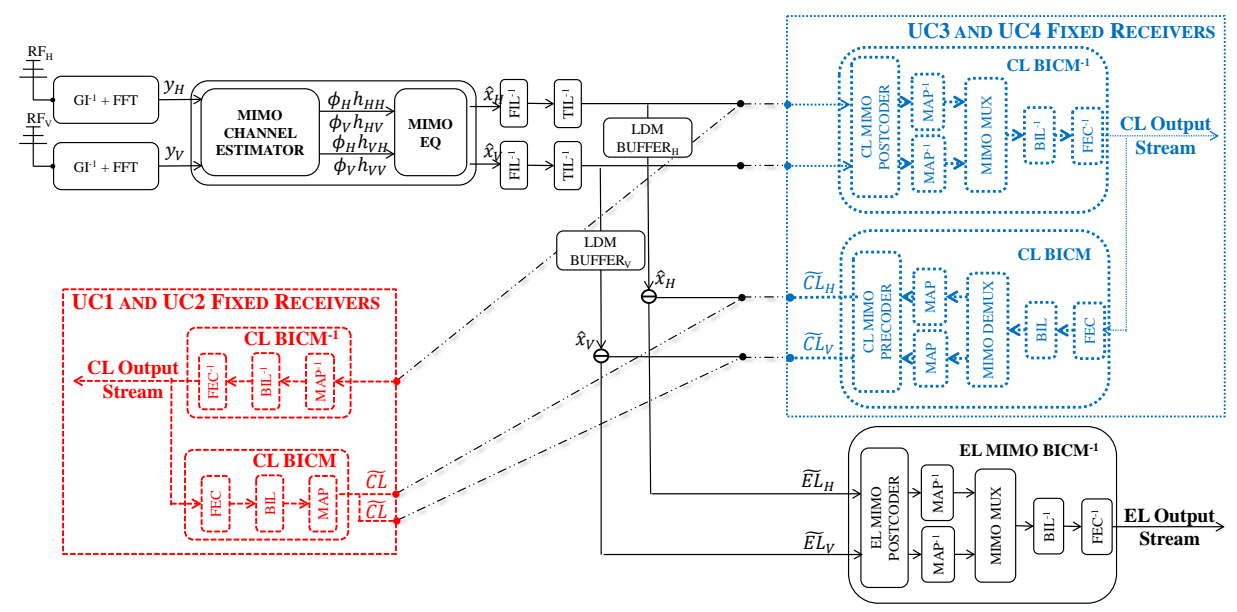

Figure 6.6: Fixed receiver block diagram for the four use cases. A MIMO channel estimator, a MIMO equalizer, two FDIL and TDIL are needed before BICM demodulation. Two LDM buffers are needed for the CL cancellation. Red-dashed: CL demodulation and remodulation for $\mathrm{UC} 1 / \mathrm{UC} 2$ with baseline BICM chains. Blue-dotted: $\mathrm{CL}$ demodulation and remodulation for $\mathrm{UC} 3 / \mathrm{UC} 4$ with $\mathrm{MIMO} \mathrm{BICM}^{-1}$ chains.

\subsubsection{Receivers blocks complexity}

The memory and/or latency requirements of the different blocks involved in the demodulation of a received signal with the different implementations are analyzed in this section. They are compared with a baseline SISO receiver.

\section{Channel Estimator}

In order to obtain the Channel Frequency Response (CFR), channel estimator makes use of pilot carriers. First, an estimation at pilot positions is done. Next, time interpolation followed by frequency interpolation is performed. Channel estimator memory requirements will depend, thus, on the pilot pattern assumed (i.e. pilot density) and the implemented time/frequency interpolation method. The widely used Least Square (LS) estimation, with a linear time interpolation and a FFT frequency interpolation is assumed in this section. Worst case has been supposed, where two memory blocks are used in order to avoid memory conflicts. 


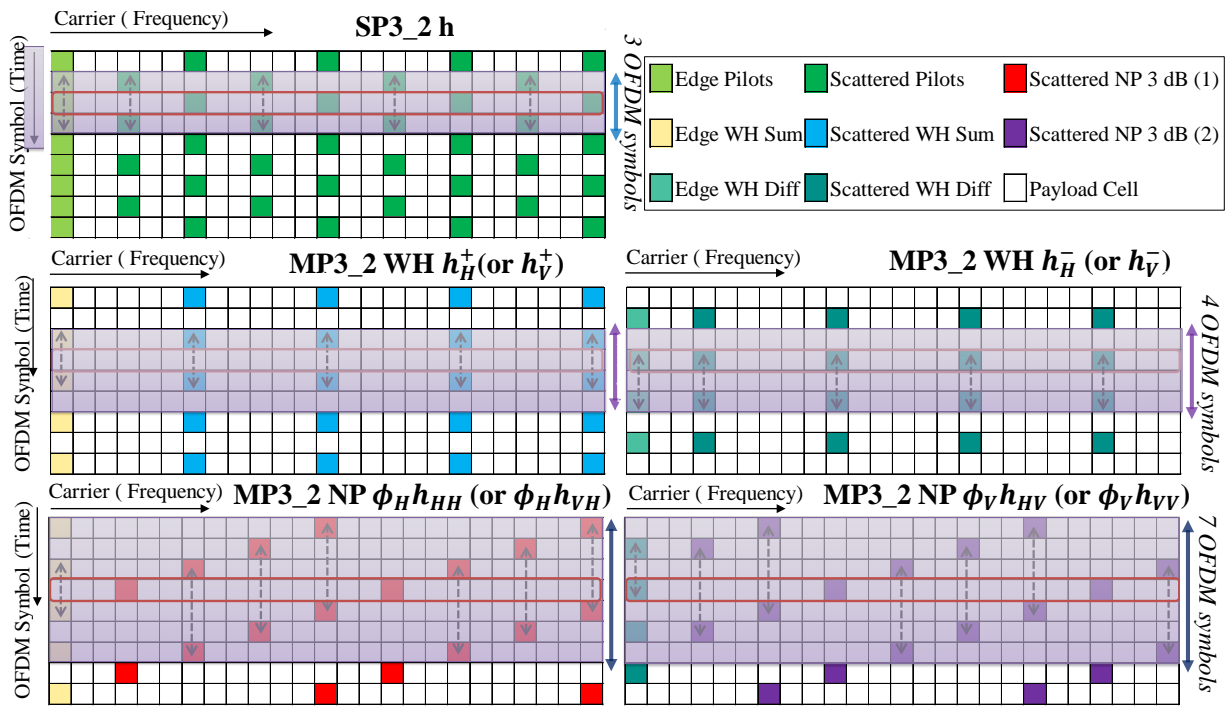

Figure 6.7: Number of OFDM symbols to be stored for a channel estimator with linear time interpolation. For SISO SP3_2, 3 OFDM symbols are required. For MIMO MP3_2 with WH, 4 OFDM symbols are required ( 3 from $h_{H}^{+}$, and 1 from $h_{H}^{-}$). For MIMO MP3_2 with NP, 7 OFDM symbols are needed.

Figure 6.7 shows the number of OFDM symbols to be stored by a linear time interpolation estimator with a SISO SP3_2, and a MIMO MP3_2 with WH and NP encodings. The memory requirements differs among them:

- SISO: $2 D_{y}-1$ OFDM symbols are needed in order to obtain the inbetween time interpolation estimates. In addition, all active carriers of each OFDM symbol should be stored. Hence, for the worst case $\left(D_{y}=4\right.$, FFT $\left.16 \mathrm{k}^{1}\right)$, a SISO receiver should be able to store $2 \cdot 7 \cdot 13825=193550$ subcarriers.

- MISO WH: In this case, the receiver observes from the first pilot subsets the sum of $h_{H}^{+}=\phi_{H} h_{H H}+\phi_{V} h_{H V}\left(\right.$ or $\left.h_{V}^{+}=\phi_{H} h_{V H}+\phi_{V} h_{V V}\right)$, whereas the second pilot subsets the difference of $h_{H}^{-}=\phi_{H} h_{H H}-\phi_{V} h_{H V}$ (or $\left.h_{V}^{-}=\phi_{H} h_{V H}-\phi_{V} h_{V V}\right)$. Thus, two parallel channel estimation are performed. Finally, WH decoding process for obtaining $\phi_{H} h_{H H}$ and $\phi_{V} h_{H V}$

\footnotetext{
${ }^{1}$ ATSC 3.0 does not allow the use of $D_{y}=4$ to $32 \mathrm{k}$ FFT size.
} 
Table 6.3: Channel Estimator memory requirements (in subcarriers)

\begin{tabular}{ccccc}
\hline \hline SISO & MISO WH & MIMO WH & MISO NP & MIMO NP \\
\hline $2 \times 96775$ & $2 \times 110600$ & $4 \times 110600$ & $2 \times 207375$ & $4 \times 207375$ \\
\hline \hline
\end{tabular}

(or $\phi_{H} h_{V H}$ and $\phi_{V} h_{V V}$ ) is performed as:

$$
\begin{cases}\phi_{H} h_{H H}=\frac{h_{H}^{+}+h_{H}^{-}}{2}, \phi_{V} h_{H V}=\frac{h_{H}^{+}-h_{H}^{-}}{2}, & \text { if } \mathrm{RF}_{\mathrm{H}} \\ \phi_{H} h_{V H}=\frac{h_{V}^{+}+h_{V}^{-}}{2}, \phi_{V} h_{V V}=\frac{h_{V}^{+}-h_{V}^{-}}{2}, & \text { if } \mathrm{RF}_{\mathrm{V}}\end{cases}
$$

The only difference with respect to SISO estimator is that for the WH case, $2 D_{y}$ OFDM symbols are needed. Thus, $2 \cdot 8 \cdot 13825=221200$ subcarriers. This corresponds to a $14,3 \%$ memory increase with respect to SISO estimators.

- MIMO WH: The memory requirements obtained above are doubled (due to the two receiving antennas).

- MISO NP: In this case, the MISO receiver observes from the first pilots subset $\phi_{H} h_{H H}\left(\right.$ or $\left.\phi_{H} h_{V H}\right)$, whereas from the second pilots subset $\phi_{V} h_{H V}\left(\right.$ or $\left.\phi_{V} h_{V V}\right)$. Hence, again, two parallel channel estimations are performed although no WH decoding is needed. As it can be observed from Figure 6.7, the number of OFDM symbols in each subset is doubled compared to SISO. Thus, $4 D_{y}-1$ OFDM symbols are needed. Overall, the MISO NP requires of storing $2 \cdot 15 \cdot 13825=414750$ subcarriers, which doubles the memory increase with respect to SISO case.

- MIMO NP: The memory requirements obtained above are doubled (due to the two receiving antennas).

Table 6.3 quantifies the number of subcarriers to be stored for the worst case with SISO and each MIMO pilot encoding. As it can be seen, whereas MIMO WH channel estimator increases in slightly more than 2 times the memory requirements compared to SISO, MIMO NP requires of more than 4 times subcarriers to be stored. This value can be considered prohibitive, as it requires almost two times ATSC 3.0 TDIL memory $\left(2^{19}=524288\right.$ cells $)$, which has been traditionally considered as the most memory demanding receiver block by manufacturers. 


\section{Equalizer}

The equalizer makes use of the channel components estimated earlier in order to derive the transmitted signal. Again, the requirements vary with the number of receiving antennas.

A baseline receiver in a SISO transmission only requires of one channel component. If a linear equalizer is considered, such as Zero Forcing (ZF), or Minimum Mean Square Error (MMSE), the equalization process is very simple [111]. It just requires of a division of the receiving symbol by the associated channel component estimated. Hence, the number of operations is $\mathcal{O}(n)$.

For the UC1/UC2, two CFRs have been estimated by mobile receivers $\left(\phi_{H} \tilde{h}_{H H}\right.$ and $\phi_{V} \tilde{h}_{H V}$, or $\phi_{H} \tilde{h}_{V H}$ and $\left.\phi_{V} \tilde{h}_{V V}\right)$. The EGC equalizer only needs of an additional operation compared to a SISO equalizer. Before the linear equalization process takes place, the two channel components are aggregated. Therefore, the number of operations remains at $\mathcal{O}(n)$ per equalized symbol. The EGC equalization processing is described below:

$$
\begin{aligned}
& \hat{x}_{H}=W_{H} y, \begin{cases}W_{H}=\frac{\left(\phi_{H} \tilde{h}_{H H}+\phi_{V} \tilde{h}_{H V}\right)^{*}}{\left|\phi_{H} \tilde{h}_{H}+\phi_{V} \tilde{h}_{H V}\right|^{2},} & \text { for ZF } \\
W_{H}=\frac{\left(\left(\phi_{H} \tilde{h}_{H H}+\phi_{\varphi_{H}} \tilde{h}_{H V}\right)^{*}\right.}{\left|\phi_{H} \tilde{h}_{H H}+\phi_{V} \tilde{h}_{H V}\right|^{2}+\sigma_{n}^{2}}, & \text { for MMSE }\end{cases} \\
& \hat{x}_{V}=W_{v} y, \begin{cases}W_{H}=\frac{\left(\left(\phi_{H} \tilde{h}_{V H}+\phi_{V} \tilde{h}_{V V}\right)^{*}\right.}{\left|\phi_{H} \tilde{h}_{V H}+\phi_{V} \tilde{h}_{V V}\right|^{2},} & \text { for ZF } \\
W_{H}=\frac{\left(\left(\phi_{H} \tilde{h}_{V H}+\phi_{V} \tilde{h}_{V V}\right)^{*}\right.}{\left|\phi_{H} \tilde{h}_{V H}+\phi_{V} \tilde{h}_{V V}\right|^{2}+\sigma_{n}^{2}}, & \text { for MMSE }\end{cases}
\end{aligned}
$$

For the mobile receivers of $\mathrm{UC} 3 / \mathrm{UC} 4$, and for all the fixed receivers, a matrix inversion operation due to the MIMO channel estimation is required:

$$
\left[\begin{array}{l}
\hat{x}_{H} \\
\hat{x}_{V}
\end{array}\right]=\left[\begin{array}{ll}
\phi_{H} \tilde{h}_{H H} & \phi_{V} \tilde{h}_{H V} \\
\phi_{H} \tilde{h}_{V H} & \phi_{V} \tilde{h}_{V V}
\end{array}\right]^{-1}\left[\begin{array}{l}
y_{H} \\
y_{V}
\end{array}\right]
$$

This matrix inversion requires of $\mathcal{O}\left(n^{3}\right)$ operations per cell. However, this number can be even higher if the inverse matrix does not exist. In this case, the Moore-Penrose pseudo-inverse can act as a partial replacement, which requires of an additional multiplication compared to regular matrix inversion. In summary, for $2 \times 2 \mathrm{MIMO}$ schemes, the number of operations per cell compared to SISO is increased from 2 to 8 .

Regarding latency and memory requirements, the channel estimator is providing to the equalizer the four channel components on a per-OFDM symbol base. Hence, the latter should be capable of perform matrix pseudo-inversion more rapidly in order to avoid overflows. 


\section{FDIL and TDIL}

FIL operates over the number of data cells in an OFDM symbol $\left(N_{C}^{D}\right)$ [112]. $N_{C}^{D}$ depends on the FFT size, coefficient reduction factor $C_{\text {red coeff }}$, and the SP. The maximum $N_{C}^{D}$ is obtained, therefore, for $32 \mathrm{k}$ FFT size, $\bar{C}_{\text {red coeff }}=0$, and SP32_2. For this combination $N_{C}^{D}=27023$ cells must be de-interleaved. Mobile receivers of $\mathrm{UC1} / \mathrm{UC} 2$ do not require of extra memory for FDIL with respect to a SISO implementation. Nevertheless, MIMO receivers will require two FDIL.

Similar procedure can be applied to TDIL memory requirements. For MIMO implementations, there are two parallel and identical TIL. Hence, TDIL requires twice the memory as for SISO. ATSC 3.0 has adopted a TDIL memory size of $M_{T I}=2^{19}$ cells for SISO. In that case, MIMO receiver will need $M_{T I}=2^{20}$ cells

\section{$B I C M^{-1}$}

Mobile receivers of $\mathrm{UC1} / \mathrm{UC} 2$ perform a baseline BICM demodulation process. It is constituted by a demapping process $\left(\mathrm{MAP}^{-1}\right.$ ) followed by a Bit De-Interleaver (BDIL) and a FEC decoder. The main restriction will arise from the number of distances to be computed at the $\mathrm{MAP}^{-1}$. However, the CL is intended to use a low constellation order, such as Quadrature PhaseShift Keying (QPSK). For this constellation, a Maximum Likelihood (ML) demapper only computes 4 distances per constellation symbol.

On the other hand, mobile receivers of UC3/UC4 and fixed receivers require of a MIMO post-coding and two parallel SISO $\mathrm{MAP}^{-1}$. Regarding MIMO postcoder, there are no particular memory needs at receiver since the three stages act on a pair of output constellation symbols. For the $\mathrm{MAP}^{-1}$, since EL is aimed at high data rates transmissions, high modulation orders are commonly used. In this case, for e.g. $64 \mathrm{NUC}$ or $256 \mathrm{NUC}$, an ML demapper will compute 64 or 256 distances per constellation symbol and antenna. 
Table 6.4: MIMO and LDM receivers complexity summary

\begin{tabular}{|c|c|c|c|}
\hline & $\begin{array}{l}\text { Mobile Rx } \\
\text { UC1/UC2 }\end{array}$ & $\begin{array}{l}\text { Mobile Rx } \\
\text { UC3/UC4 }\end{array}$ & $\begin{array}{c}\text { Fixed Rx } \\
\text { UC1 } / \mathbf{U C 2} / \mathbf{U C 3} / \mathrm{UC4}\end{array}$ \\
\hline Channel & $114,3 \%(\mathrm{WH})$ & $228,6 \%(\mathrm{WH})$ & $228,6 \%(\mathrm{WH})$ \\
\hline Estimator & $214,3 \%(\mathrm{NP})$ & $428,6 \%(\mathrm{NP})$ & $428,6 \%(\mathrm{NP})$ \\
\hline \multirow{2}{*}{ Equalizer } & SISO & MIMO & MIMO \\
\hline & $\mathcal{O}(n)$ & $\mathcal{O}\left(n^{3}\right)$ & $\mathcal{O}\left(n^{3}\right)$ \\
\hline FDIL & SISO & $2 \times \mathrm{SISO}$ & $2 \times \mathrm{SISO}$ \\
\hline TDIL & SISO & $2 \times \mathrm{SISO}$ & $2 \times \mathrm{SISO}$ \\
\hline LDM Buffer & - & - & $2 \times \mathrm{LDM}$ \\
\hline $\mathrm{BICM}^{-1}$ & SISO & $2 \times \mathrm{SISO}$ & $2 \times$ SISO \\
\hline
\end{tabular}

\section{LDM buffers}

All previous blocks are inherent to the multiple antenna scheme used in the transmission, and are not directly related with LDM mode. If LDM is also used, additional complexity is found in the LDM cancellation process at fixed receivers.

LDM buffer needs to store the de-interleaved LDM aggregated symbols until the CL cancellation is conducted. LDM buffer size highly depends on constellation order and FEC block length of CL and EL. The maximum buffer size in order to avoid memory conflicts between the current FEC block and the next incoming FEC block is 64800 constellation symbols [51]. Moreover, for fixed MIMO receivers two parallel and independent CL cancellation processes are needed. Hence, two LDM buffers are required. In this case, the maximum memory LDM buffer is doubled to 129600 cells. LDM buffers memory size for the worst case is about $12.4 \%$ of $M_{T I}$. Hence, it does not represent a serious constraint for fixed receivers design.

Regarding latency requirements, CL FEC Blocks should be demodulated, remodulated, and cancelled faster than the TDIL read out rate. If the TDIL read out rate is faster than this latency, the LDM buffer is overflowed and the EL signal cannot be properly demodulated.

Table 6.4 summarizes the complexity of each receiver block for the four use cases under evaluation. From the table it can be observed that the complexity 
increase mainly comes from the use of co-located multiple antenna schemes rather than LDM. Mobile receivers with one antenna (on UC1/UC2) will only require a more complex channel estimator compared to baseline receivers. Mobile receivers with two antennas (UC3/UC4), will double memory size of almost every receiver block. The additional requirements from LDM are associated to fixed receivers. They required of LDM buffers for the CL cancellation. Thus, for every fixed receiver, the joint MIMO transmission with LDM should include all MIMO demands and LDM buffers. In order to limit memory demands for every UC, MIMO WH pilot encoding is recommended, as NP encoding requires more than two times channel estimator size.

\subsection{Methodology and Simulation Setup}

The performance of LDM and co-located MIMO schemes is evaluated by means of physical layer simulations. A validated software simulator for the SISO baseline scheme, which is updated with the new MIMO and LDM blocks, is assumed. The assumed transmitter setup, channel models, and receiver configuration are explained below.

\subsubsection{Transmitter Setup}

The four use cases are evaluated for the two LDM layers. In order to provide a fair comparison in terms of data-rate, the LDM signal is constituted by a CL QPSK 4/15 stream for UC1/UC2, and two QPSK 2/15 streams for UC3/UC4. For the EL, two 64 NUC $10 / 15$ streams are transmitted in every UC. TDCFS filtering with a filter length of $L=256$ samples is enabled for UC2 and UC4 in both LDM layers. The rest of configurable parameters are common for all the use cases. Table 6.5 summarizes the different configurations assumed for the performance evaluation.

\subsubsection{Channel models}

Three different channel models are evaluated. An ideal Additive White Gaussian Noise (AWGN) channel with realistic Cross-Polarization Discrimination (XPD) factors for mobile reception, XPD $=6 \mathrm{~dB}$, and for fixed-rooftop reception, $\mathrm{XPD}=20 \mathrm{~dB}$ is assessed. In addition, MIMO channel models extracted from broadcasting field test campaigns are also evaluated. In particular, for CL performance, the DVB-NGH mobile channel model[113] with a Doppler 
Table 6.5: Transmitter Setup

\begin{tabular}{|c|c|c|c|c|}
\hline \multicolumn{5}{|c|}{ USE CASE PARAMETERS } \\
\hline \multirow{2}{*}{$U C 1$} & Core Layer & Enhanced Layer & \multirow{2}{*}{$\Delta=4 \mathrm{~dB}$} & TDCFS \\
\hline & $1 \times \mathrm{QPSK} 4 / 15$ & $2 \times 64 \mathrm{NUC} 10 / 15$ & & $O F F$ \\
\hline \multirow{2}{*}{ UC2 } & Core Layer & Enhanced Layer & \multirow{2}{*}{$\Delta=4 \mathrm{~dB}$} & TDCFS \\
\hline & $1 \times \mathrm{QPSK} 4 / 15$ & $2 \times 64 \mathrm{NUC} 10 / 15$ & & $O N$ \\
\hline \multirow{2}{*}{ UC3 } & Core Layer & Enhanced Layer & \multirow{2}{*}{$\Delta=4 \mathrm{~dB}$} & TDCFS \\
\hline & $2 \times$ QPSK $2 / 15$ & $2 \times 64 \mathrm{NUC} 10 / 15$ & & $O F F$ \\
\hline \multirow{2}{*}{$U C_{4}$} & Core Layer & Enhanced Layer & \multirow{2}{*}{$\Delta=4 \mathrm{~dB}$} & TDCFS \\
\hline & $2 \times$ QPSK $2 / 15$ & $2 \times 64$ NUC $10 / 15$ & & $O N$ \\
\hline \multicolumn{5}{|c|}{ WAVEFORM PARAMETERS } \\
\hline \multirow{2}{*}{$T I L$} & \multicolumn{2}{|c|}{ TIL Type } & \multicolumn{2}{|c|}{ TIL Size } \\
\hline & \multicolumn{2}{|c|}{ S-PLP Convolutional } & \multicolumn{2}{|c|}{1024 rows } \\
\hline \multirow{2}{*}{$M P$} & \multirow{2}{*}{$\mathrm{MP} 6 \_2$} & \multirow{2}{*}{ Boosting 4} & \multirow{2}{*}{\multicolumn{2}{|c|}{$\begin{array}{c}\text { Walsh Hadamard } \\
\text { Null Pilots }\end{array}$}} \\
\hline & & & & \\
\hline \multirow{2}{*}{$O F D M$} & FFT Size & \multirow{2}{*}{$C_{\text {red_coeff }}=0$} & GI length & Bandwidth \\
\hline & $16 \mathrm{k}$ & & 1024 samples & $6 \mathrm{MHz}$ \\
\hline
\end{tabular}

spread $f_{D}=33,3 \mathrm{~Hz}$ is considered, while for EL performance, the Modified Guilford Model (MGM) channel [114] is assumed.

\subsubsection{Receiver Setup}

A LS estimation with a moving average or linear time interpolation and an FFT frequency interpolation [110] is considered for the channel estimator. In addition, an MMSE equalizer is assumed. 


\subsection{LDM and co-located MIMO Performance Evaluation}

This section studies the potential gains offered by the joint configuration of co-located MIMO schemes with LDM. The studies are divided in three main sections. A preliminary evaluation of the Mean Square Error (MSE) of the channel estimation for the DVB-NGH and MGM channel models is assessed. It provides a comparison between the two MP encodings (WH and NP), as well as between different time interpolation methods in practical mobile and fixed scenarios. The CL performance is evaluated next. The evaluation of the mobile receivers with one antenna ( $\mathrm{UC} 1$ and $\mathrm{UC} 2$ ) is compared with the performance of UC3 and UC4, i.e. for mobile receivers with two antennas. Last, the EL performance for the four use cases is evaluated. The EL MIMO SM performance evaluation is assessed when the CL is transmitted as a MISO and a MIMO scheme.

\subsubsection{Mean Square Error of Channel Estimation}

The MSE of channel estimator is evaluated in order to select the most suitable implementation to be used by mobile receivers for the CL, and by fixed receivers for the EL. Three time interpolation methods are considered, linear and two Moving Average window lengths (distance between pilot bearing carriers in time domain, and the total number of OFDM symbols of the current subframe). Figure 6.8 illustrates the MSE for DVB-NGH (top) and MGM (bottom).

\section{DVB-NGH channel}

It can be observed that NP outperforms $\mathrm{WH}$ at low SNR regions, i.e. at noise-limited regions. This is because of the $3 \mathrm{~dB}$ boosting pilot power of NP. Nevertheless, when the noise variance is negligible, the Inter-Carrier Interference (ICI) becomes the most dominant parameter in time selective channels. Hence, the denser MP pattern in time domain (WH with $D_{y}=2$ versus NP with $\left.D_{y}=4\right)$ should provide a more accurate estimation. This is confirmed from SNR $\geq 8 \mathrm{~dB}$, where it is observed that WH provides a lower MSE. Regarding the most suitable implementation, the shortest time-window length is recommended, which corresponds to linear interpolation among the three options. Last, the additional TDCFS frequency selectivity/diversity has no impact on the channel estimation error. 

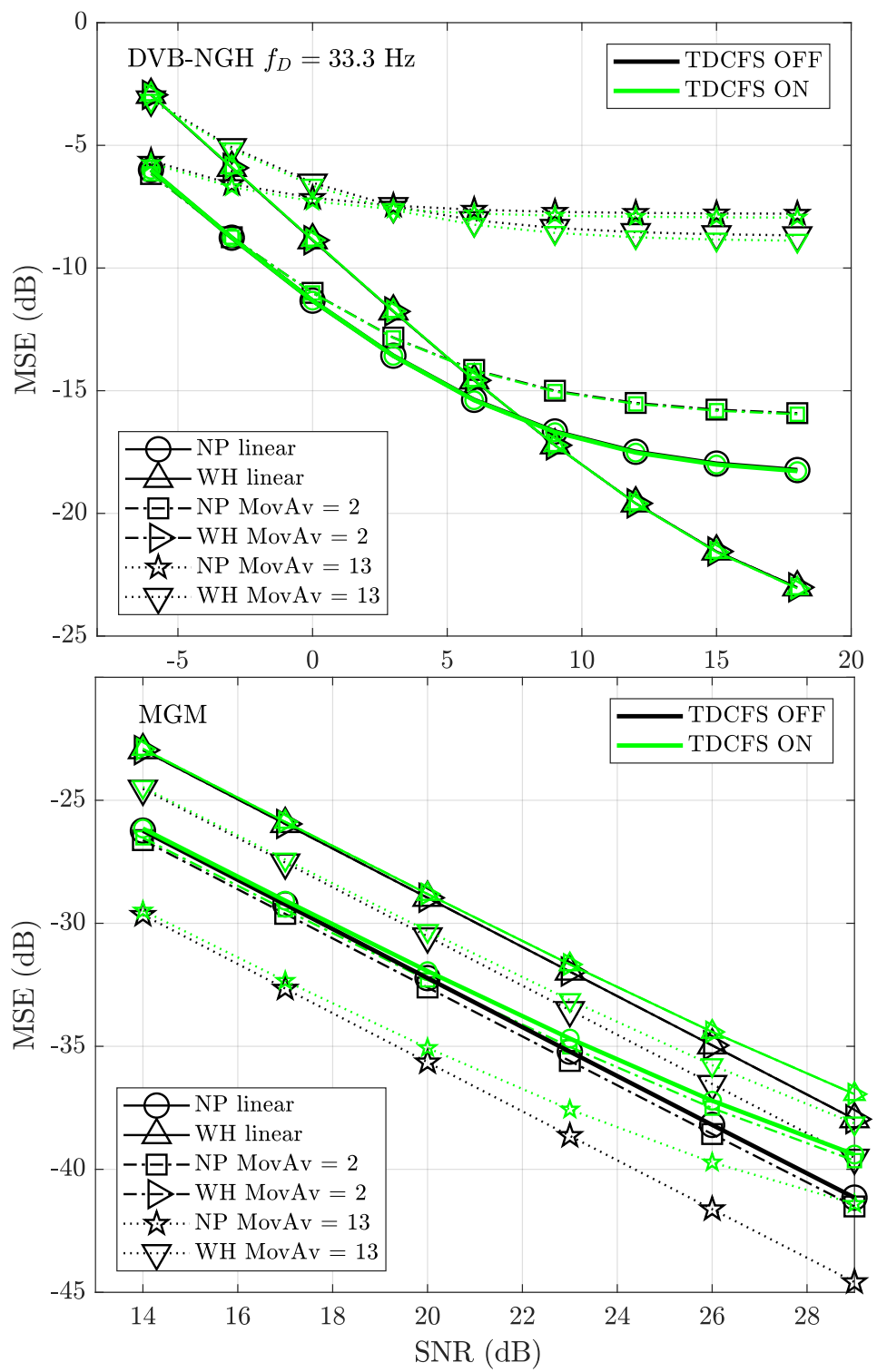

Figure 6.8: MSE estimation for DVB-NGH mobile $\left(f_{D}=33,3 \mathrm{~Hz}\right)$, and MGM channel for NP and WH with different time interpolation methods and with and without TDCFS. 


\section{MGM channel}

Contrary to previous channel, MGM is a non-time variant channel. Thus, at high SNR regions, the Inter-Symbol Interference (ISI) becomes as the most restrictive effect. It can be seen that for this channel, NP provides the lowest MSE regardless of the SNR operational region. For low SNR regions, as in DVB-NGH channel, it is due to the $3 \mathrm{~dB}$ increased pilot power. In contrast, at high SNR regions, it is due of a denser pattern in frequency domain (whereas on NP $D_{x}=6$, in WH it is extended to $D_{x}=12$ ). Regarding the most suitable time interpolation method, since this is a time-invariant channel, the longest time-window length will provide the lowest MSE. This can be explained by multi-rate digital signal processing, where the resultant noise bandwidth is reduced for longer interpolation factors. From the different evaluated configurations, Moving Average with 13 OFDM symbols is the longest window length, and, consequently, provides the lowest MSE. Last, it can be observed that TDCFS has an impact on the MSE. In particular, it is increased when TDCFS is enabled. This can be explained by the higher frequency selectivity introduced by TDCFS. It prevents deep fadings by increasing the frequency diversity, but at the same time, a denser pattern is required to follow the channel fluctuations.

In summary, NP outperforms WH in almost all the reception conditions under consideration. Nevertheless, it requires of a much higher memory increase compared to WH. Since performance results are still needed in order to provide a final recommendation, next sections still consider the two MP encodings. Regarding time interpolation, a linear interpolation is only assumed for the CL performance, and the Moving Average with $\mathrm{N}=13$ OFDM symbols is employed for the EL performance.

\subsubsection{Performance}

This subsection aims to evaluate the CL performance for all the use cases. In order to provide a fair comparison with $\mathrm{UC} 1$ and $\mathrm{UC} 2$ results in terms of spectral efficiency, the CL code rate has been halved from $1 \times$ QPSK $4 / 15$ to $2 \times \mathrm{QPSK} 2 / 15$ for $\mathrm{UC} 3 / \mathrm{UC} 4$. 


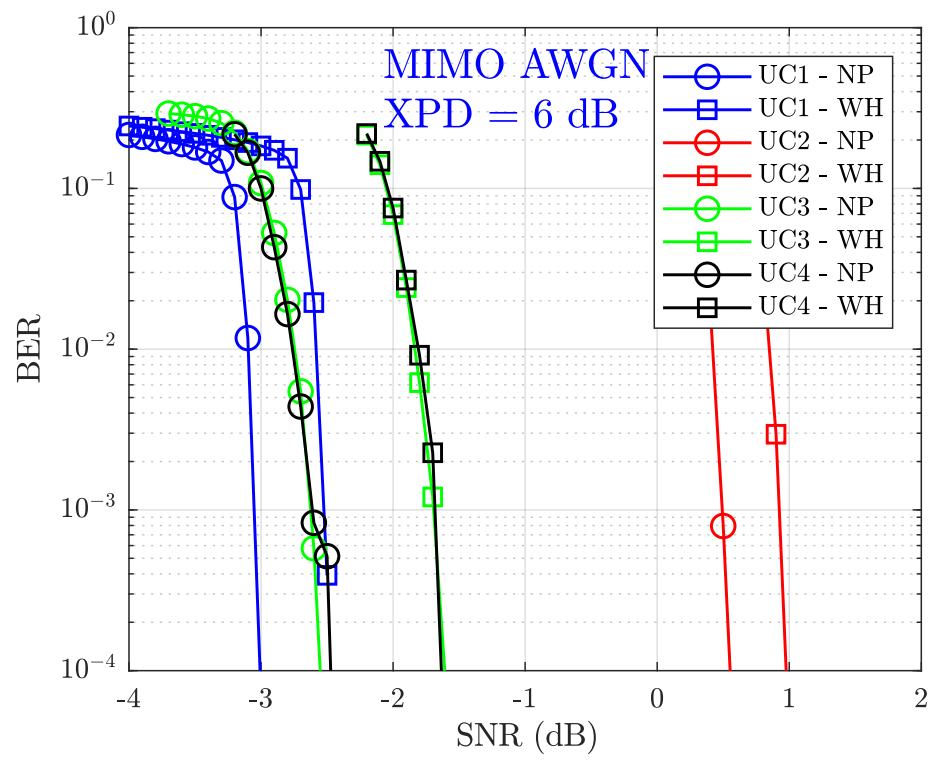

Figure 6.9: CL BER performance for MIMO AWGN with XPD $=6 \mathrm{~dB}$ for all use cases with Null Pilots and Walsh Hadamard MP encodings.

\section{MIMO AWGN $(X P D=6 d B)$}

Figure 6.9 depicts the Bit Error Rate (BER) for all the use cases with the two MP encodings under AWGN channel. It can be observed that UC1 with NP encoding outperforms the rest of configurations, including UC3. The diversity and multiplexing trade-off explains this behaviour. Whereas the MISO diversity gains are increased with the correlation between channel paths, the spatial multiplexing gains of MIMO SM are decreased [115]. For the MIMO AWGN channel under study, the correlation between paths is virtually obtained by the XPD factor, so that the highest correlation is obtained for XPD $=0 \mathrm{~dB}$. A medium value, such as the assumed XPD $=6 \mathrm{~dB}$, is still providing higher diversity gains than multiplexing gains. It can also be seen that, as expected from previous section, NP outperforms WH regardless of the UC, thanks to the $3 \mathrm{~dB}$ boosting pilot power. Last, it can be noticed that the $\mathrm{UC} 2$ performance, is highly decreased with respect to $\mathrm{UC1}$ (up to $3.5 \mathrm{~dB}$ ). This is because the TDCFS filters in an AWGN channel are enhancing rather than frequency diversity, the frequency selectivity, as it shown in Figure 6.10. 


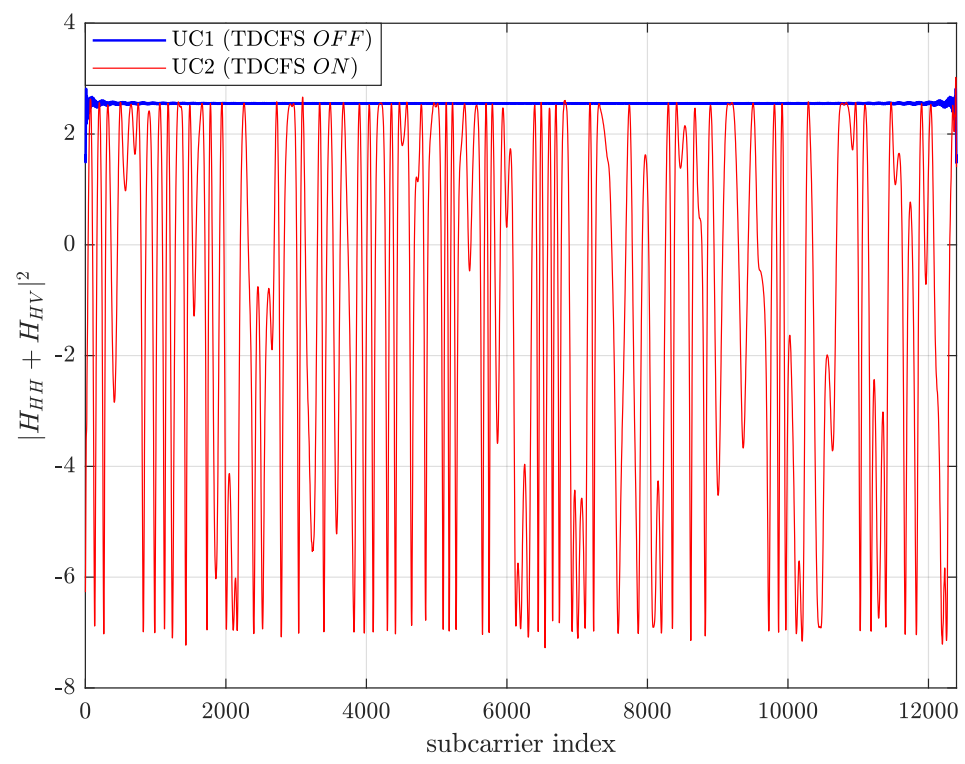

Figure 6.10: Estimated CFR for UC1 and UC2 for AWGN (XPD $=6 \mathrm{~dB}$ ) with NP encoding.

\section{DVB $\boldsymbol{N G H}\left(f_{D}=33.3 \mathbf{H z}\right)$}

The CL performance for all the use cases with the DVB-NGH mobile channel at $f_{D}=33.3 \mathrm{~Hz}$ is provided in Figure 6.11. As it can be observed, due to lack of frequency diversity of this channel, $\mathrm{UC1}$ is no longer providing the previous spatial diversity gains. This is translated into a significant performance decrease with respect to AWGN channel. On the other hand, UC3 and UC4 are now exploiting in a higher factor the spatial multiplexing gains as the operational SNR of the system has been increased. This also helps to understand the MP encoding comparison. It can be seen that the performance gap between $\mathrm{NP}$ and $\mathrm{WH}$ is reduced for $\mathrm{UC2} 2 \mathrm{UC} 4$, where the SNR thresholds are increased with respect to previous channel model. Moreover, for the SNR threshold of $\mathrm{UC1}$ ( $\mathrm{SNR} \approx 13 \mathrm{~dB}$ ), WH is outperforming NP thanks to its denser pattern in time-domain. Last, it can be noticed that MISO TDCFS of UC2 is giving a similar performance as MIMO SM use cases. This is because it is now increasing the frequency diversity and preventing deep fadings. Figure 6.12 shows the estimated CFR for UC1 and UC2, where the additional frequency diversity of TDCFS is shown. 


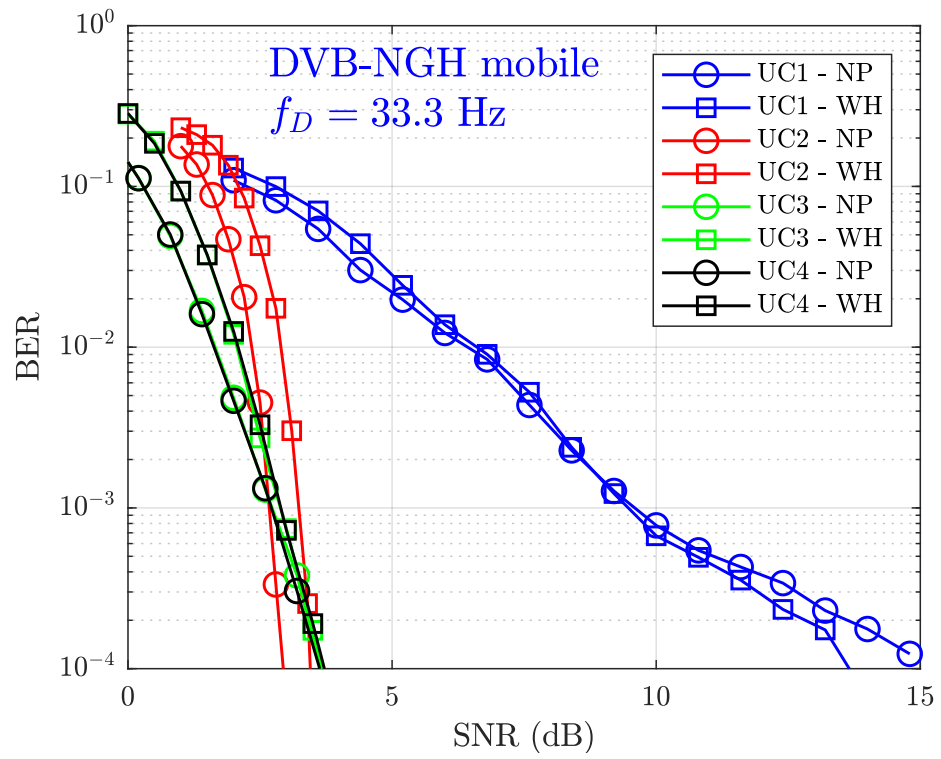

Figure 6.11: CL BER performance for DVB-NGH mobile channel with $f_{D}=33.3 \mathrm{~Hz}$ for all use cases with Null Pilots and Walsh Hadamard MP encodings.

Table 6.6 summarizes the CL SNR thresholds at BER $=10^{-4}$ with the four use cases. It can be observed that use cases with MIMO SM (UC3 and UC4) do not outperform in a high degree the two MISO use cases (UC1 and UC2). The spatial multiplexing gain of using a lower Low Density Parity Check (LDPC) coding rate can be caught up by the spatial and frequency diversity of MISO schemes. Therefore, the performance gains achieved by MIMO SM can be considered insufficient for the CL, taking into account the required complexity increase at mobile receivers. Finally, when the two MP encodings are analyzed it can be observed that NP generally outperforms WH. However, these gains are in a range lower than $1 \mathrm{~dB}$ for every channel evaluated. Thus, considering the memory requirements of NP at channel estimator, WH is recommended. 


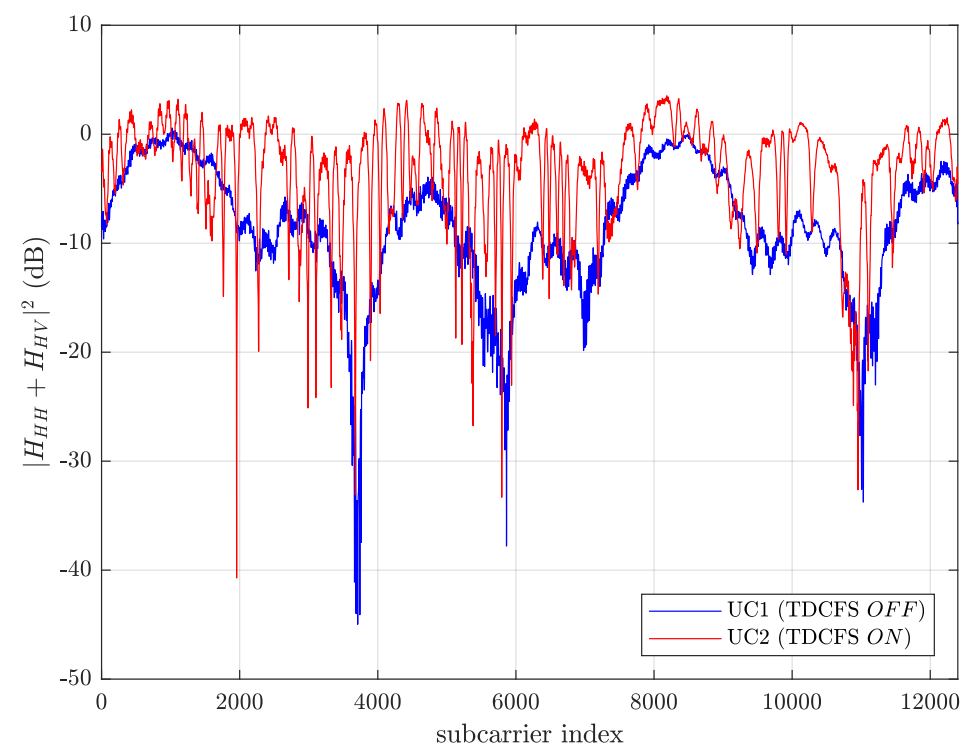

Figure 6.12: Estimated CFR for UC1 and UC2 for DVB-NGH mobile channel $\left(f_{D}=33.3\right.$ $\mathrm{Hz}$ ) with NP encoding.

\subsubsection{EL Performance}

From the CL performance results of previous section, UC1 or UC2 has been recommended. They provided similar performance as UC3 or UC4 but with less complexity at mobile receivers. This subsection evaluates the EL performance for all the use cases in order to quantify the impact of using a MISO scheme on the CL. Since the EL operational SNR region is much higher than the required for the CL, no impact in performance is expected from using MISO or MIMO schemes in the CL. The BER versus SNR is provided in Figure 6.13 for all the use cases with the two MP encodings and the two fixed channel models.

Two conclusions can be extracted from the results. First, the impact of using a MISO scheme on the CL does not affect to the EL performance. For the same MP encoding, the performance differences among the four use cases are negligible for both channel models. Second, NP encoding outperforms WH at most $0.5 \mathrm{~dB}$. On the one hand, for the MIMO AWGN, the SNR thresholds of the four use cases at BER $=10^{-4}$ are $18.7 \mathrm{~dB}$ and $19.2 \mathrm{~dB}$ for NP and WH encoding, respectively. On the other hand, $10 \mathrm{~dB}$ of performance degradation is observed for the more challenging MGM channel. The SNR thresholds at $\mathrm{BER}=10^{-3}$ range from $27.2 \mathrm{~dB}$ to $28.3 \mathrm{~dB}$. Table 6.7 summarizes the EL 
Table 6.6: CL SNR thresholds at BER $=10^{-4}$ for the two MP encodings in MIMO AWGN channel with XPD $=6 \mathrm{~dB}$, and DVB-NGH mobile channel with $f_{D}=33.3 \mathrm{~Hz}$.

\begin{tabular}{cccccc}
\hline \hline & & $U C 1$ & UC2 & UC3 & UC4 \\
\hline$A W G N$ & $N P$ & $-3.0 \mathrm{~dB}$ & $0.6 \mathrm{~dB}$ & $-2.5 \mathrm{~dB}$ & $-2.5 \mathrm{~dB}$ \\
\cline { 2 - 6 }$(X P D=6 \mathrm{~dB})$ & $W H$ & $-2.5 \mathrm{~dB}$ & $1.0 \mathrm{~dB}$ & $-1.6 \mathrm{~dB}$ & $-1.6 \mathrm{~dB}$ \\
\hline$D V B-N G H$ mobile & $N P$ & $15.0 \mathrm{~dB}$ & $3.0 \mathrm{~dB}$ & $3.7 \mathrm{~dB}$ & $3.7 \mathrm{~dB}$ \\
\cline { 2 - 6 }$\left(f_{D}=33.3 \mathrm{~Hz}\right)$ & $W H$ & $13.6 \mathrm{~dB}$ & $2.5 \mathrm{~dB}$ & $3.7 \mathrm{~dB}$ & $3.7 \mathrm{~dB}$ \\
\hline \hline
\end{tabular}

Table 6.7: EL SNR thresholds for the two MP encodings in MIMO AWGN channel XPD $=20 \mathrm{~dB}\left(\right.$ at BER $\left.=10^{-4}\right)$, and MGM channel $\left(\right.$ at BER $\left.=10^{-3}\right) . \mathrm{p}$

\begin{tabular}{cccccc}
\hline \hline & & UC1 & UC2 & UC3 & UC4 \\
\hline$A W G N$ & $N P$ & $18.7 \mathrm{~dB}$ & $18.7 \mathrm{~dB}$ & $18.7 \mathrm{~dB}$ & $18.7 \mathrm{~dB}$ \\
\cline { 2 - 6 }$(X P D=20 \mathrm{~dB})$ & $W H$ & $19.2 \mathrm{~dB}$ & $19.2 \mathrm{~dB}$ & $19.2 \mathrm{~dB}$ & $19.2 \mathrm{~dB}$ \\
\hline \multirow{2}{*}{$M G M$} & $N P$ & $27.8 \mathrm{~dB}$ & $27.2 \mathrm{~dB}$ & $27.4 \mathrm{~dB}$ & $27.4 \mathrm{~dB}$ \\
\cline { 2 - 6 } & $W H$ & $28.3 \mathrm{~dB}$ & $27.2 \mathrm{~dB}$ & $27.8 \mathrm{~dB}$ & $27.8 \mathrm{~dB}$ \\
\hline \hline
\end{tabular}

SNR thresholds with the four use cases. In summary, taking into account all the previous results, the MISO on the CL and MIMO SM on the EL is the recommended joint LDM and MIMO UC. The performance gains of MIMO SM cannot justify the complexity increase for mobile receivers. TDCFS use is also recommended in order to exploit the frequency diversity preventing deep fadings. Hence, among the different use cases, it is recommended the UC2. Regarding MP encoding, WH is recommended in order to reduce channel estimator memory requirements on mobile receivers at the expense of $0.5 \mathrm{~dB}$ performance loss.

\subsection{Conclusions}

This paper studies the joint transmission of ATSC 3.0 Layered Division Multiplexing (LDM) mode with co-located Multiple-Input Multiple-Output (MIMO) schemes. Two main use cases were proposed. The first use case, aimed at reducing mobile receivers' complexity, employed a co-located Multiple-Input Single-Output (MISO) scheme for the LDM Core Layer (CL), while the MIMO Spatial Multiplexing (MIMO SM) was only exploited in the LDM Enhanced 

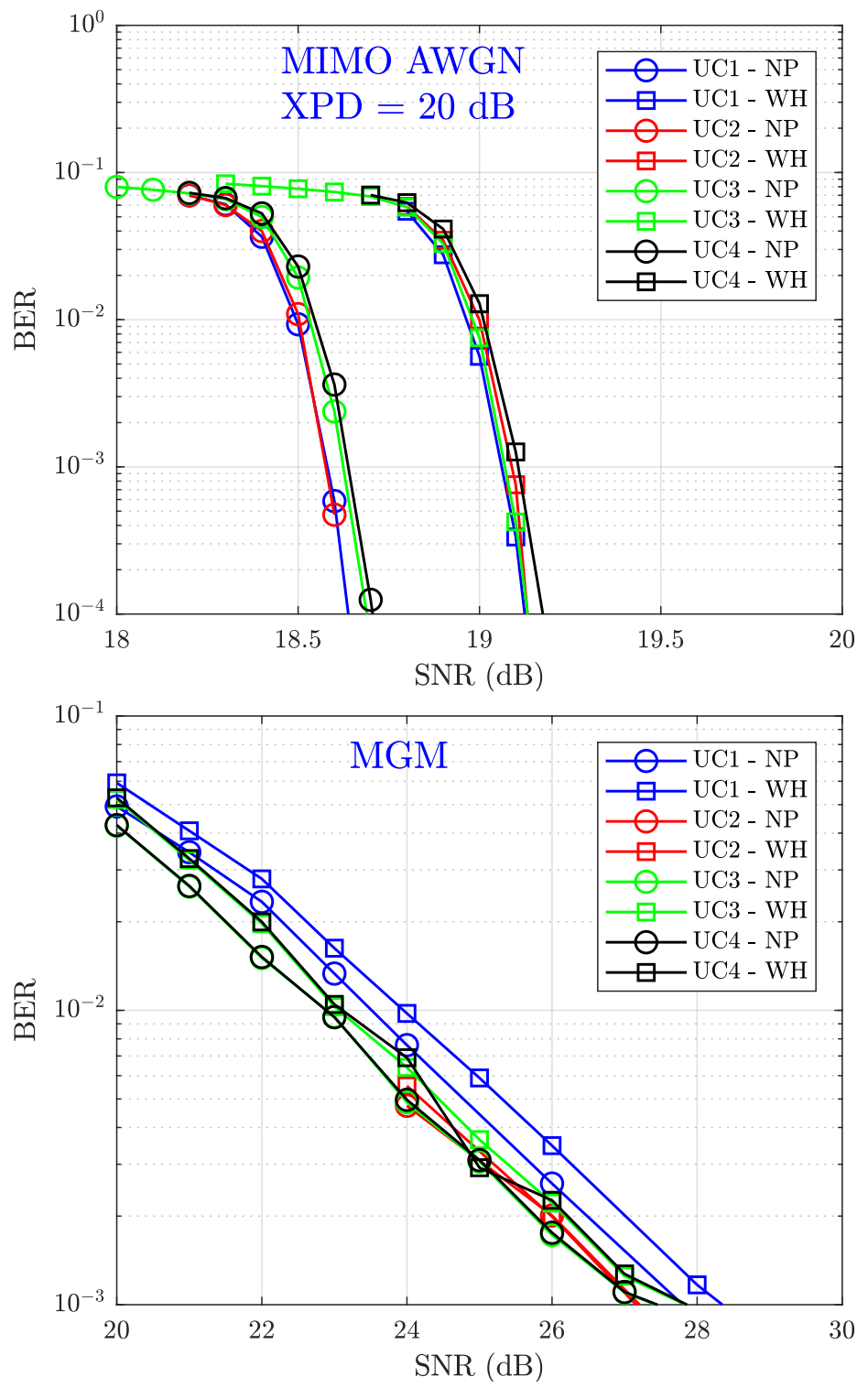

Figure 6.13: EL BER performance for MIMO AWGN (XPD = $20 \mathrm{~dB}$ ) (top) and MGM channel (bottom) for all use cases with Null Pilots and Walsh Hadamard MP encodings.

Layer (EL). The second use case, where there were no restrictions in terms of mobiles' complexity, employed a MIMO SM on both LDM layers. The imple- 
mentation aspects of the two proposed joint use cases was analyzed. It was noticed that no additional requirements other than those related to changing from SISO to MIMO transmission were needed. Special focus had been given on the channel estimator's memory. In comparison with a baseline receiver, the use of MIMO Pilot (MP) encodings increased the required channel estimator memory. The lowest memory increase was required by the Walsh Hadamard encoding (WH) in MISO receivers, with only a $14.3 \%$ the required memory increase. On the other hand, the use of Null Pilot encoding (NP) increased the memory up to 4 times for MIMO receivers with respect to baseline SISO receivers.

The performance of all the use cases was evaluated for the two LDM layers with different channel models extracted from field measurement campaigns (DVB-NGH for mobility conditions, and MGM for fixed reception) and with the two MP encodings. From the CL results, it was considered that the spatial multiplexing gains of MIMO SM were not enough for the additional complexity it entailed on mobile receivers. In addition, since the operational SNR of the CL was very low compared to the EL, the use of MISO or MIMO in the CL had no impact on the EL performance. It was also observed that NP encoding only outperformed WH about $0.5 \mathrm{~dB}$ on both layers. In summary, taking into account the complexity and performance trade-off, the MISO on the CL and MIMO SM on the EL was the recommended use case with the WH encoding. 



\section{Chapter 7}

\section{Results Discussions}

This section provides a global vision of the investigations carried out during the development of this thesis. It is divided in two main parts. First part presents a summary of the performance results of the core elements of the Layered Division Multiplexing (LDM) systems, i.e. the Core Layer (CL), the Enhanced Layer (EL). Next, the implementation aspects that should be bear in mind for mobile and fixed receivers are highlighted.

\subsection{Performance Evaluation}

Figure 7.1 illustrates an ATSC - Third Generation (ATSC 3.0) baseline transmitter block diagram when the LDM mode is used. In order to reduce receivers complexity, the CL and EL are configured with independent Bit-Interleaved Coded Modulation (BICM) chains, but they share the same Time Interleaver (TIL), Frequency Interleaver (FIL), Pilot Pattern (PP), Multiple-Input SingleOutput (MISO) algorithm, Fast Fourier Transform (FFT) size, and Guard Interval (GI). Next sections assess the impact of each of these blocks in the CL and EL performance. 


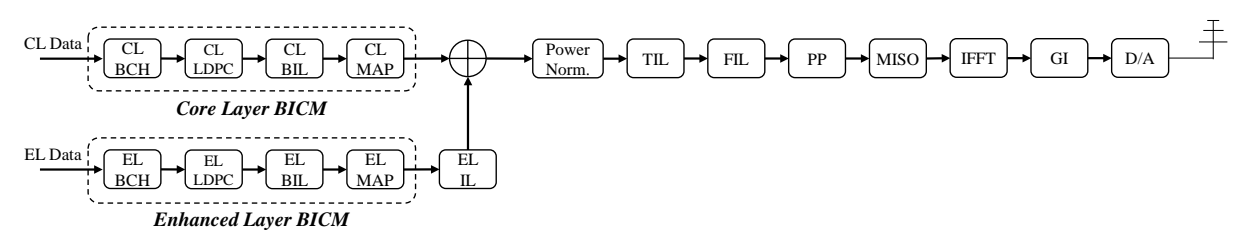

Figure 7.1: LDM transmitter block diagram.

\subsubsection{BICM Impact}

The structure of the BICM block consists of the serial concatenation of the outer encoder (Bose Chadhuri Hocquenghem (BCH)), inner encoder (Low Density Parity Check (LDPC)), a Bit Interleaver (BIL) and a modulation mapper. BICM is one of the most important modules, as it provides the error correction capability for the system, allowing the broadcaster to choose among different configurations for the desired robustness and capacity characteristics [55].

\section{CL performance}

Because of the required robustness for the correct operation of the CL, a fixed Quadrature Phase-Shift Keying (QPSK) modulation was assumed in this thesis. The efficiency of the LDPC coding rate for the CL was assessed in Chapter 2, where two different demapping algorithms were analyzed. The straightforward Gaussian Demapping (GD) approach [45], which treats the EL as Additive White Gaussian Noise (AWGN) interference was compared with an Optimum Demapping (OD) approach, which assumes the knowledge of the EL constellation. The 12 LDPC coding rates adopted in ATSC 3.0 were evaluated for AWGN and independent and identically distributed (i.i.d.) Rayleigh channel in Section 2.4.1 and Section 2.4.2, respectively. It was observed that OD performance gains depended on the operational Signal-to-Noise Ratio (SNR), which is related with the Injection Level $(\Delta)$. For low coding rates $(3 / 15-$ $7 / 15$ ), OD approach outperformed GD when the operational SNR was above $5 \mathrm{~dB}$ in AWGN. On the other hand, for high coding rates $(8 / 15-13 / 15)$, OD started to outperform GD when the operational SNR was above $10 \mathrm{~dB}$. In order to assess the same study in a more realistic scenario, an i.i.d. Rayleigh fading channel, modeling portable reception, was also considered. A general performance degradation was observed because of the more challenging conditions of the channel, and performance differences between the two demapping algorithms were obtained at a lower operational SNR. In particular, noticeable gains were observed at $0 \mathrm{~dB}$ for low coding rates and from $5 \mathrm{~dB}$ at high coding 
Table 7.1: OD gains (dB) for QPSK + QPSK in AWGN / i.i.d. Rayleigh

\begin{tabular}{cccccccc}
\hline \hline \multirow{2}{*}{$x_{t}$} & \multicolumn{7}{c}{$\rho(d B)$} \\
\cline { 2 - 7 } & 0 & 1 & 2 & 3 & 4 & 5 & 6 \\
\hline $2 / 15$ & $0.0 / 0.9$ & $0.0 / 0.4$ & $0.0 / 0.2$ & $0.0 / 0.0$ & $0.0 / 0.0$ & $0.0 / 0.0$ & $0.0 / 0.0$ \\
\hline $3 / 15$ & $0.0 / 1.8$ & $0.0 / 0.7$ & $0.0 / 0.4$ & $0.0 / 0.2$ & $0.0 / 0.0$ & $0.0 / 0.0$ & $0.0 / 0.0$ \\
\hline $4 / 15$ & $0.4 / 3.4$ & $0.1 / 1.4$ & $0.0 / 0.6$ & $0.0 / 0.4$ & $0.0 / 0.2$ & $0.0 / 0.1$ & $0.0 / 0.0$ \\
\hline $5 / 15$ & $1.2 / 12.9$ & $0.3 / 2.0$ & $0.0 / 0.8$ & $0.0 / 0.4$ & $0.0 / 0.3$ & $0.0 / 0.1$ & $0.0 / 0.0$ \\
\hline $6 / 15$ & $5.1 /-$ & $0.9 / 5.6$ & $0.2 / 1.3$ & $0.0 / 0.6$ & $0.0 / 0.3$ & $0.0 / 0.2$ & $0.0 / 0.0$ \\
\hline $7 / 15$ & $-/-$ & $1.4 /-$ & $0.3 / 2.8$ & $0.1 / 0.9$ & $0.0 / 0.3$ & $0.0 / 0.2$ & $0.0 / 0.1$ \\
\hline $8 / 15$ & $-/-$ & $-/-$ & $0.3 /-$ & $0.1 / 2.5$ & $0.0 / 0.4$ & $0.0 / 0.4$ & $0.0 / 0.2$ \\
\hline $9 / 15$ & $-/-$ & $-/-$ & $2.3 /-$ & $0.3 / 6.7$ & $0.1 / 1.0$ & $0.0 / 0.8$ & $0.0 / 0.4$ \\
\hline $10 / 15$ & $-/-$ & $-/-$ & $10.4 /-$ & $1.3 /-$ & $0.2 / 1.9$ & $0.0 / 2.2$ & $0.0 / 1.1$ \\
\hline $11 / 15$ & $-/-$ & $-/-$ & $7.4 /-$ & $5.1 /-$ & $0.9 / 6.3$ & $0.2 / 5.7$ & $0.0 / 2.3$ \\
\hline $12 / 15$ & $-/-$ & $-/-$ & $8.1 /-$ & $7.9 /-$ & $2.8 /-$ & $0.7 /-$ & $0.2 /-$ \\
\hline $13 / 15$ & $-/-$ & $-/-$ & $-/-$ & $-/-$ & $6.2 /-$ & $3.3 /-$ & $0.8 /-$ \\
\hline \hline
\end{tabular}

rates. The OD gains are summarized in Table 7.1. An additional result that was extracted from this chapter was the CL performance dependance on the EL constellation.

\section{EL performance}

The EL performance evaluation was also assessed on Chapter 2 of this thesis. In addition to the QPSK modulation, different Non-Uniform Constellation (NUC) orders (16NUC, 64NUC, and 256NUC) as well as different LDPC coding rates $(4 / 15$, and $10 / 15)$ were considered. Their efficiencies were analyzed by using the traditional Hard Cancellation (HC) method, and compared with a Soft Cancellation (SC) demapping algorithm. The latter regards the CL Log-Likelihood Ratios (LLR)s as a-priori information on the EL demapping. Thus, the traditional Successive Interference Cancellation (SIC) process could be omitted. In summary, three demapping configurations were analyzed for the EL performance: $\mathrm{GD}+\mathrm{HC}, \mathrm{OD}+\mathrm{HC}$, and $\mathrm{OD}+\mathrm{SC}$. The performance was evaluated for AWGN and for DVB Fixed Rice (DVB-F1), but similar conclusions were extracted regardless of the channel model. It was observed that the OD gains were again obtained for high CL coding rates. On the other hand, 
Table 7.2: OD and SC gains (dB) for for AWGN / DVB-F1

\begin{tabular}{cccccc}
\hline \hline \multirow{2}{*}{$C L, E L$} & \multirow{2}{*}{$\Delta(d B)$} & \multicolumn{2}{c}{$Q P S K+Q P S K$} & \multicolumn{2}{c}{$Q P S K+16 N U C$} \\
\cline { 2 - 6 } & & $O D$ & $S C$ & $O D$ & $S C$ \\
\hline \multirow{2}{*}{$10 / 15,4 / 15$} & 2 & $-/-$ & $-/-$ & $-/-$ & $-/-$ \\
\cline { 2 - 6 } & 4 & $-/-$ & $-/-$ & $-/-$ & $-/-$ \\
\hline \multirow{2}{*}{$10 / 15,10 / 15$} & 2 & $\infty / \infty$ & $0.1 / 0.1$ & $\infty / \infty$ & $0.1 / 0.1$ \\
\cline { 2 - 6 } & 4 & $0.2 / 0.7$ & $0.1 / 0.1$ & $0.0 / 0.0$ & $0.0 / 0.0$ \\
\hline \hline \multirow{2}{*}{$C L, E L$} & \multirow{2}{*}{$\Delta(d B)$} & $Q P S K$ & $64 N U C$ & $Q P S K$ & $256 N U C$ \\
\cline { 2 - 6 } & & $O D$ & $S C$ & $O D$ & $S C$ \\
\hline \multirow{2}{*}{$10 / 15,4 / 15$} & 2 & $\infty / \infty$ & $3.9 / 3.8$ & $\infty / \infty$ & $5.3 / 5.1$ \\
\cline { 2 - 6 } & 4 & $0.2 / 1.0$ & $0.1 / 0.2$ & $0.0 / 0.0$ & $0.0 / 0.0$ \\
\hline \multirow{2}{*}{$10 / 15,10 / 15$} & 2 & $\infty / \infty$ & $0.4 / 0.3$ & $\infty / \infty$ & $0.2 / 0.2$ \\
\cline { 2 - 6 } & 4 & $0.2 / 0.2$ & $0.0 / 0.0$ & $0.0 / 0.0$ & $0.0 / 0.0$ \\
\hline \hline
\end{tabular}

the benefits of SC approach were only observed when a low coding rate was employed in the EL, and varied depending on the EL modulation order. Table 7.2 summarizes the EL SNR thresholds with the different configurations.

In summary, it is concluded that the Optimum Demapping gains are not really attractive to ATSC 3.0 LDM configuration. Nevertheless, it may be useful in systems which employing robust signals with low power differences, such as Wideband reuse-1 (WiB) systems. Moreover, it is considered that the combination of Optimum Demapping with Soft-Cancellation can highly improve the performance of the underlying signal.

\subsubsection{TIL Impact}

The TIL has been considered as an integral part of the physical layer to mitigate long burst errors, which can greatly degrade the correction capabilities of Forward Error Correction (FEC) coding. Thus, TIL increases time diversity and transmission robustness at the expense of latency and zapping times [30]. ATSC 3.0 adopted a convolutional type for Single - PLP (S-PLP) mode and a hybrid type for Multiple PLP (M-PLP). The impact of the TIL depths of each type were investigated on mobility conditions on Section 3.5.1 and on 
Table 7.3: $2 \mathrm{RF}+$ TIL gains for different correlation factors

\begin{tabular}{cccc}
\hline Correlation & \multicolumn{3}{c}{ Freq.+Time Diversity gains $(\mathrm{dB})$} \\
\cline { 2 - 4 }$\rho$ & $0 \Rightarrow 50 \mathrm{~ms}$ & $50 \Rightarrow 100 \mathrm{~ms}$ & $100 \Rightarrow 200 \mathrm{~ms}$ \\
\hline 0 & 8.7 & 6.1 & 3.5 \\
\hline 0.3 & 8.4 & 5.7 & 3.2 \\
\hline 0.5 & 7.9 & 5.3 & 2.8 \\
\hline 0.7 & 6.5 & 4.6 & 2.1 \\
\hline 0.9 & 3.9 & 3.1 & 1.1 \\
\hline 11 & 0 & 0 & 0 \\
\hline
\end{tabular}

Section 4.6.2. In addition, a performance comparison between them and also with the Digital Video Broadcasting - Next Generation Handheld (DVB-NGH) block type was analyzed in Section 4.6.1 for the EL when Multi-Radio Frequency Channel (MultiRF) techniques are also used.

\section{CL performance}

Three TIL depths of the convolutional type were evaluated in Section 3.5.1 for different receiver speeds. Figure 3.4 depicted the SNR thresholds of the different FFT sizes, TIL depths and speeds under study. From the figure, it was shown that $1 \mathrm{~dB}$ gain was achieved by doubling the TIL depth, at lowmedium speeds and for all the FFT sizes. These speeds are characterized by a large coherence time, resulted in a lack of time diversity, which is the main goal of TIL. However, when the receiver speed was increased to the Inter-Carrier Interference (ICI)-limited zone, the benefits for using a longer TIL depth were reduced.

The impact of the hybrid TIL depth was also investigated in Section 4.6.2. It was evaluated when MultiRF techniques were also employed. It was observed for different correlation factors between Radio Frequency (RF) channels and at different receiving speeds. Three main conclusions were extracted from the research. First, it was stood out that the correlation factor affected the time as well as the frequency diversities. The higher the correlation between $2 \mathrm{RF}$ channel realizations, the lower the TIL and MultiRF gains (see Figure 4.8. Table 7.3 summarizes the diversity gains for different correlation factors at $v=3 \mathrm{~km} / \mathrm{h}$ speed. 
Table 7.4: Performance losses $(d B)$ for the different TIL schemes.

\begin{tabular}{cccc}
\hline \hline RF-Mux & Hybrid TIL & Conv TIL & DVB-NGH Block TIL \\
\hline 2 RF channels & 0 & 0.18 & 0.51 \\
\hline 4 RF channels & 0 & 1.08 & 1.24 \\
\hline 6 RF channels & 0 & 1.36 & 1.86 \\
\hline \hline
\end{tabular}

Next, it was also confirmed that the higher diversity gains were achieved for lower speeds. However, since these results were obtained under ideal channel estimation conditions, the ICI could not be observed. Finally, it was also observed that MultiRF gains were directly related with the TIL depth. The higher the TIL depth, the lower the MultiRF gains. This is explained by the diminishing marginal returns of diversity concept introduced in [46]. As time diversity is firstly obtained with the TIL, the frequency diversity gains of MultiRF techniques were marginal.

\section{EL performance}

Although the main objective of the TIL is aimed at improving CL performance in low-mobility conditions, the impact on the EL was also carried out in this thesis. The proper distribution of cells among the RF channels on a TimeFrequency Slicing (TFS) transmission depended on the TIL type. Thus, since the two LDM layers share the same TIL type, the TFS gains for the EL could be affected. This was firstly devised in Section 4.4.1, where the ATSC 3.0 hybrid TIL reached the best even distribution of cells. Finally, it was confirmed with performance results in Section 4.6.1. Table 7.4 summarizes the performance loss of the three TIL schemes under evaluation with respect to the ideal performance.

In summary, it is recommended to use the $200 \mathrm{~ms}$ time interleaving depth in order to improve the CL pedestrian performance $(v=3$ $\mathrm{km} / \mathrm{h}$ ) up to $13 \mathrm{~dB}$. 


\subsubsection{PP Impact}

In broadcast transmissions there is no return channel. Thus, an accurate channel estimation at receivers is of utmost importance for the proper data demodulation. In order to estimate the radio channel properly, some Orthogonal Frequency-Division Multiplexing (OFDM) carriers, which are already known by receivers, are multiplexed between the data carriers. These carriers are known as Scattered Pilot (SP) carriers. The channel state is firstly estimated in the SP and afterwards is interpolated in the time and frequency domains across the other carriers and OFDM symbols. Therefore, there are two terms that will affect the estimation accuracy of the channel state, the transmitted pilot configuration and the interpolation processing implemented at receivers.

As it occurred with the TIL, the LDM layers share the same pilot configuration, which conducts to a new trade-off between robustness and capacity. Whereas the CL demands a dense pilot configuration, the EL requires the minimum capacity overhead. Thus, the selection of the optimum pilot configuration is not so blatant. In addition, ATSC 3.0 provides up to 16 different SP patterns, with up to 5 different amplitudes for each one, known as pilot boostings. The evaluation of the different SP patterns and pilot boostings for Time Division Multiplexing (TDM) systems and for the LDM two layers was assessed in Section 3.5.2 and Section 3.5.3, respectively. An extract of the SNR thresholds for LDM layers with different configurations is provided in Table 7.5. It was recommended SP6_2 with boosting 1 as an optimum SP configuration. Same SP pattern was recommended in [104]. A brief explanation of the obtained results is discussed next.

\section{CL performance}

The impact of SP density and SP amplitude on the CL performance was assessed for a Typical Urban (TU-6) with two Doppler shifts, $33 \mathrm{~Hz}$ and $55 \mathrm{~Hz}$. These Doppler shifts correspond to user speeds $v=60 \mathrm{~km} / \mathrm{h}$, and $v=100$ $\mathrm{km} / \mathrm{h}$ for a carrier frequency $f_{c}=600 \mathrm{MHz}$, respectively. It was observed that increasing the SP density in frequency domain only improved the performance for the pilot boosting 0 . Nevertheless, this pilot boosting provided the worst performance. Therefore, in order to reduce the capacity overhead without affecting the channel estimation accuracy, it was recommended to use at least a pilot boosting 1 with a SP pattern not very dense. 
Table 7.5: SNR thresholds (dB) of CL and EL for different pilot configurations with $\Delta=4$ dB.

\begin{tabular}{cccccc}
\hline \hline & \multicolumn{3}{c}{$C L$} & \multicolumn{2}{c}{$E L$} \\
\hline SP & $S P$ & $T U-6$ & $T U-6$ & DVB-F1 & 0 dB echo \\
Pattern & Boosting & $f_{d}=33 \mathrm{~Hz}$ & $\left.f_{d}=55 \mathrm{~Hz}\right)$ \\
\hline & 0 & 6.1 & 6.6 & 19.4 & 21.9 \\
\cline { 2 - 6 } SP3_2 & 1 & 6.0 & 6.6 & 19.4 & 21.9 \\
\cline { 2 - 6 } & 4 & 6.0 & 6.4 & 19.8 & 22.3 \\
\hline & 0 & 6.5 & 6.5 & 19.4 & 21.8 \\
\cline { 2 - 6 } SP6_2 & 1 & 6.1 & 6.0 & 19.5 & 21.8 \\
\cline { 2 - 6 } & 4 & 6.1 & 6.0 & 19.8 & 22.1 \\
\hline & 0 & 6.7 & 6.4 & 19.4 & 21.9 \\
\cline { 2 - 6 } SP12_2 & 1 & 6.1 & 5.9 & 19.5 & 21.9 \\
\cline { 2 - 6 } & 4 & 6.1 & 6.0 & 19.8 & 22.0 \\
\hline & 0 & 7.4 & 7.5 & 19.7 & 22.1 \\
\cline { 2 - 6 } SP32_2 & 1 & 6.4 & 6.0 & 19.5 & 21.9 \\
\cline { 2 - 6 } & 4 & 6.4 & 6.0 & 19.6 & 21.9 \\
\hline \hline
\end{tabular}

\section{EL performance}

The impact of SP density and SP amplitude on the EL performance was assessed for a DVB-F1 and $0 \mathrm{~dB}$ echo channel (50\% GI). They can be associated with a fixed-reception condition in a Multiple Frequency Network (MFN) and a Single Frequency Networks (SFN) topology, respectively. In comparison with CL, same trend regarding SP density was observed. Using a denser SP pattern only improves the performance for pilot boosting 0 . However, when the pilot boostings were compared it was observed that pilot boosting 0 provided the best performance. 

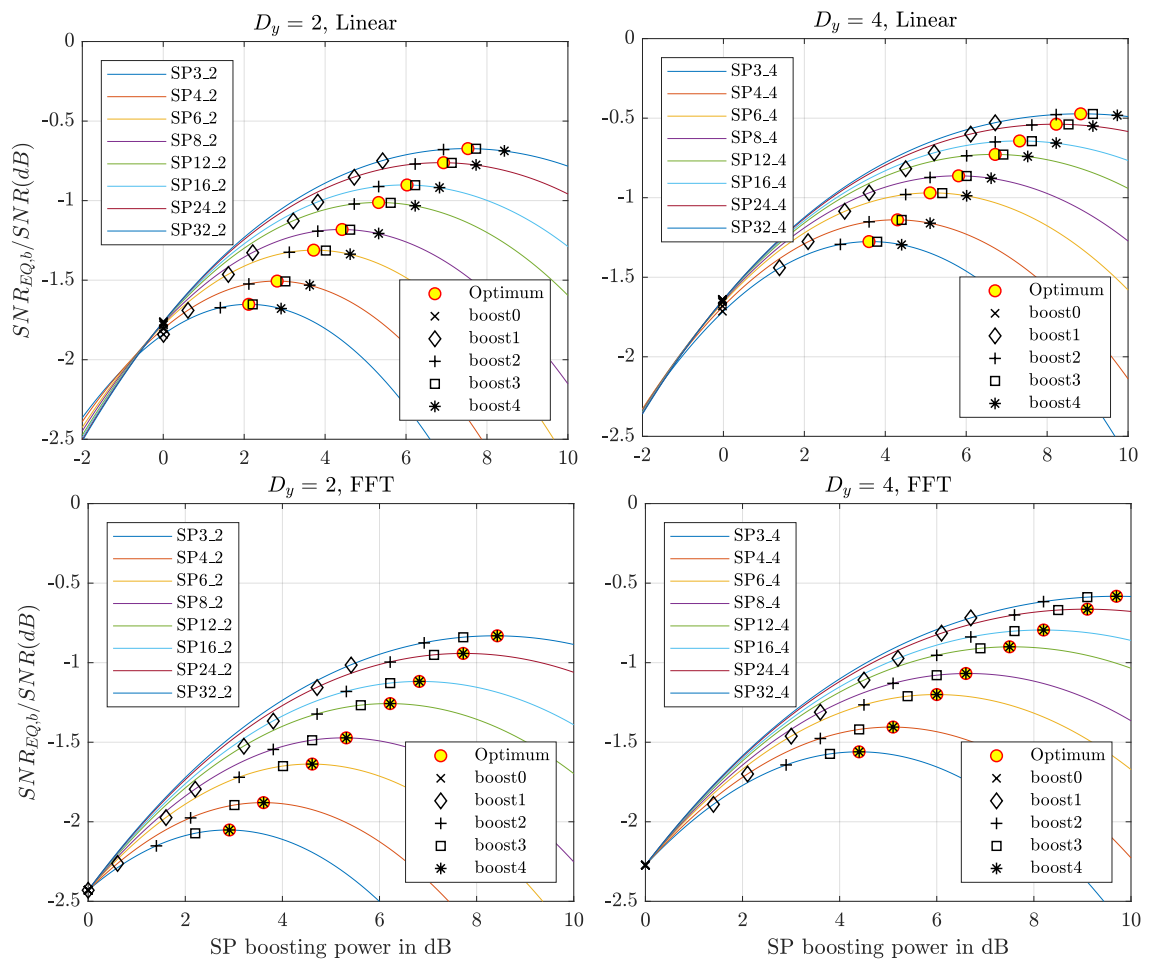

Figure 7.2: Equalized SNR performance with linear (top) and FFT (bottom) frequency interpolator.

\section{Injection Level $(\Delta)$ impact}

When LDM performance was compared with TDM, small differences were noticed at the fixed service, i.e. at the EL. They were explained due to the Cross-Layer Interference (CLI), dragged by a bad CL cancellation. It was observed that this CLI factor increased with the $\Delta$, but on a small scale.

\section{Channel Estimator impact}

It should be noted that the results are very dependant on the channel estimator implementation. The above results were obtained with a Least Square (LS) estimator at pilot positions followed by a linear interpolation in time domain and a Wiener interpolation in the frequency domain [87]. If a different channel 
estimator is implemented, the conclusions of this paper could differ. In particular, the adopted pilot boosting values were obtained by using Equation (7.1), where $f_{\text {int }}$ is the noise reduction factor by time and frequency interpolation (which was described in publication [ST2] of Section 1.6.

$$
S N R_{E Q, b}=\frac{\sigma_{s}^{2} \times k}{\sigma_{N}^{2}+\sigma_{N}^{2} \times f_{\text {int }} / b}=S N R \times \frac{k}{1+f_{\text {int }} / b}
$$

Figure 7.2 presents the adopted pilot boosting values in ATSC 3.0 and the optimum when a linear (top) or a FFT (bottom) frequency interpolator are implemented. From the figure it can be seen that, whereas the optimum pilot boosting for linear frequency interpolator is pilot boosting 3, for a FFT frequency interpolator is pilot boosting 4 .

In summary, since no significant gains are achieved by using a denser pilot pattern in both LDM layers, the Scattered Pilot Pattern SP6 2 is recommended. In another vein, pilot boosting 4 is finally recommended, as the FFT frequency interpolation method is the most commonly used in the market.

\subsubsection{MISO Impact}

Distributed MISO schemes are used in terrestrial broadcast to reduce SFN self-destructive interferences, and hence, increase the coverage area. Two distributed MISO schemes have been adopted in next-gen Digital Terrestrial Television (DTT) standards: Transmit Diversity Code Filter Sets (TDCFS), a frequency pre-distortion algorithm, in ATSC 3.0, and Alamouti, a SpaceFrequency Block Code (SFBC) algorithm, in DVB - Terrestrial Second Generation (DVB-T2) and DVB-NGH. The impact of the two distributed MISO schemes on the LDM systems was firstly analyzed in [110], which corresponds to Chapter 5 of this thesis. Since MISO Alamouti scheme requires of orthogonal PP between transmitters, the ATSC 3.0 MIMO Pilot (MP) encodings Walsh-Hadamard (WH) and Null Pilot (NP) were used. Seven SFN scenarios were considered. They varied according to the number of transmitters and the reception delays, but same amplitudes were assumed. These are also known as $0 \mathrm{~dB}$ echo scenarios. The performance results are discussed next. 


\section{CL performance}

A TU-6 realization associated to a Doppler shift $f_{D}=33.3 \mathrm{~Hz}$ was applied to each transmitter path for the CL performance. Small performance differences were observed between MISO Alamouti with NP encoding, MISO TDCFS and not using any distributed MISO scheme. This was explained due to the diminishing marginal returns of diversity concept again. The time diversity provided by the TIL limits the gains associated to subsequent blocks, such as the spatial diversity of distributed MISO. Regarding the comparison among the different scenarios, it was noticed that since the diversity increases with the number of transmitters, the overall performance of the three schemes also did so.

\section{EL performance}

In order to observe the most destructive SFN interference in fixed reception conditions, uncorrelated realizations with same magnitude but different random phase were applied to each transmitter path. For this layer, it was easier to distinguish the spatial diversity of distributed MISO schemes, as time diversity could not be achieved with the TIL. On the one hand, MISO Alamouti outperformed up to $3 \mathrm{~dB}$ the other two configurations for scenarios with two transmitters. These differences were reduced with the number of transmitter to $1.7 \mathrm{~dB}$ gains due to the frequency selectivity. On the other hand, regarding using MISO TDCFS, only gains were observed at null echo delay. This is, in fact, the scenario for what it was originally designed. For the rest of scenarios, no performance difference were detected.

To sum up, Table 7.6 presents the SNR threshold at Bit Error Rate (BER) = $10^{-4}$ for the LDM configuration CL QPSK 4/15, EL 64NUC 10/15, $\Delta=4 \mathrm{~dB}$. A wider range of $\Delta=\{2-6\} \mathrm{dB}$, but similar conclusions were extracted. Thus, it was considered that it has not impact on the distributed MISO gains.

Overall, on the one hand the use of distributed MISO schemes do not provide relevant gains on mobility performance. Moreover, TDCFS does neither provide significant gains on fixed scenarios. On the other hand, up to $3 \mathrm{~dB}$ gains can be obtained in fixed receivers if MISO Alamouti is employed. Thus, it is recommended for LDM transmissions, where mobile and fixed services are simultaneously delivered. Therefore, the distributed MISO Alamouti scheme is proposed to be include into the next DTT standard. 
Table 7.6: CL and EL SNR threshold $(\mathrm{dB})$ at $\mathrm{BER}=10^{-4}$ for all the scenarios under evaluation

\begin{tabular}{|c|c|c|c|c|c|c|}
\hline \multirow[b]{2}{*}{ Scenario } & \multicolumn{3}{|c|}{ CL performance } & \multicolumn{3}{|c|}{ EL performance } \\
\hline & $\begin{array}{c}\text { MISO } \\
\text { Alamouti }\end{array}$ & $\begin{array}{c}M I S O \\
T D C F S\end{array}$ & $S F N$ & $\begin{array}{c}\text { MISO } \\
\text { Alamouti }\end{array}$ & $\begin{array}{c}M I S O \\
T D C F S\end{array}$ & $S F N$ \\
\hline $\begin{array}{c}2 T x \\
(\tau=0 \% G I)\end{array}$ & 4.0 & 4.2 & 5.1 & 18.8 & 22.4 & NA \\
\hline $\begin{array}{c}2 T x \\
(\tau=1.3 \% G I)\end{array}$ & 4.0 & 4.2 & 4.1 & 18.8 & 22.4 & 21.7 \\
\hline $\begin{array}{c}2 T x \\
(\tau=90 \% G I)\end{array}$ & 3.9 & 4.1 & 4.1 & 19.0 & 21.7 & 21.7 \\
\hline $3 T x$ & 3.6 & 3.8 & 3.8 & 19.4 & 21.1 & 21.1 \\
\hline $4 T x$ & 3.1 & 3.4 & 3.4 & 19.7 & 21.5 & 21.4 \\
\hline $5 T x$ & 2.9 & 3.2 & 3.3 & 19.7 & 21.6 & 21.5 \\
\hline $8 T x$ & 2.7 & 3 & 3 & 20.1 & 21.8 & 21.7 \\
\hline
\end{tabular}

\subsubsection{FFT Impact}

ATSC 3.0 is based on the OFDM modulation. OFDM mitigates the effects of Inter-Symbol Interference (ISI) of frequency selective channels by dividing a broadband RF channel into a set of narrowband channels. Nevertheless, it becomes vulnerable to fast time-varying channels, i.e. to ICI originated by Doppler shifts. The Doppler tolerance depends on the carrier spacing, and, therefore, on the FFT size of the system. For the same RF bandwidth, the smaller the FFT size, the higher the carrier spacing, and, so, the stronger the Doppler tolerance. ATSC 3.0 has adopted three different FFT sizes (8k, $16 \mathrm{k}$, and $32 \mathrm{k}$ ). Chapter 3 evaluates the FFT size impact for the CL under mobility conditions. A TU-6 channel model with a wide range of Doppler shifts $\left(f_{D}=11,17,22,33,44,55,83\right.$, and $\left.111 \mathrm{~Hz}\right)$ was assumed. Table 7.7 shows the SNR thresholds for the three FFT sizes at BER $=10^{-4}$ with a QPSK 4/15, and TIL $=200 \mathrm{~ms}$. From the table, a better performance was initially observed when the Doppler shift was increased. Nevertheless, after this performance increase thanks to the time diversity, the higher FFT sizes started to suffer a performance degradation. In particular, whereas $8 \mathrm{k}$ FFT only increased its performance, $16 \mathrm{k}$ and $32 \mathrm{k}$ performance decreased from $f_{D}=83 \mathrm{~Hz}$ and 
Table 7.7: SNR thresholds $(\mathrm{dB})$ at BER $=10^{-4}$ of a QPSK $4 / 15$ for the the three FFT sizes and different Doppler shifts.

\begin{tabular}{ccccccccc}
\hline \hline \multirow{2}{*}{ FFT } & \multicolumn{8}{c}{ Doppler $(\mathrm{Hz})$} \\
\cline { 2 - 9 } & 11 & 17 & 22 & 33 & 44 & 55 & 83 & 111 \\
\hline $8 k$ & 2.0 & 1.4 & 1.1 & 0.5 & 0.1 & 0.0 & -0.3 & -0.5 \\
\hline $16 k$ & 2.2 & 1.5 & 1.0 & 0.6 & 0.3 & 0.2 & 0.5 & 2.2 \\
\hline $32 k$ & 1.8 & 1.7 & 1.1 & 1.1 & 1.4 & 2.6 & NA & NA \\
\hline \hline
\end{tabular}

$f_{D}=44 \mathrm{~Hz}$, respectively. In Section 3.3, it was defined the Doppler limit $\left(f_{D, \text { limit }}\right)$ as a function of the FFT size, GI length, and the length of the SP in OFDM symbols, $\left(D_{y}\right)$ as:

$$
f_{D, \text { limit }}=\frac{1}{2 D_{y} \cdot\left(T_{U}+T_{G}\right)} H z
$$

where $T_{U}$ was the useful symbol duration, and $T_{G}$ was the GI length in time. The corresponding $f_{D, \text { limit }}$ for the three configurations under study were: $187 \mathrm{~Hz}$ for $8 \mathrm{k}, 92 \mathrm{~Hz}$ for $16 \mathrm{k}$, and $50 \mathrm{~Hz}$ for $32 \mathrm{k}$. These values matched with the simulation results of Table 7.7.

In summary, 16k FFT size is recommended to be used in LDM. It will allow the proper demodulation of the CL at speeds up to 165 $\mathrm{km} / \mathrm{h}$ in the Ultra-High Frequency (UHF) band (600 MHz), while the GI overhead is halved in comparison with 8k FFT size. It is considered that this speed is more than sufficient for most vehicular receivers.

\subsubsection{MIMO Impact}

Compared to a baseline Single-Input Single-Output (SISO) receiver with one antenna at the transmitter and the receiver side, the utilization of multiple transmit and/or receive antennas can provide three performance gains. Spatial diversity gain due to the averaging of fading across different channel paths. Array gain due to the coherent combination of signals at the receiver side. Spatial multiplexing gain by increasing the transmitted data rate. These gains are achieved at the expense of a cost increase in receiver side, due to the mandatory implementation of multiple antennas. ATSC 3.0 has adopted the Multiple-Input Multiple-Output (MIMO) scheme implemented with two co- 


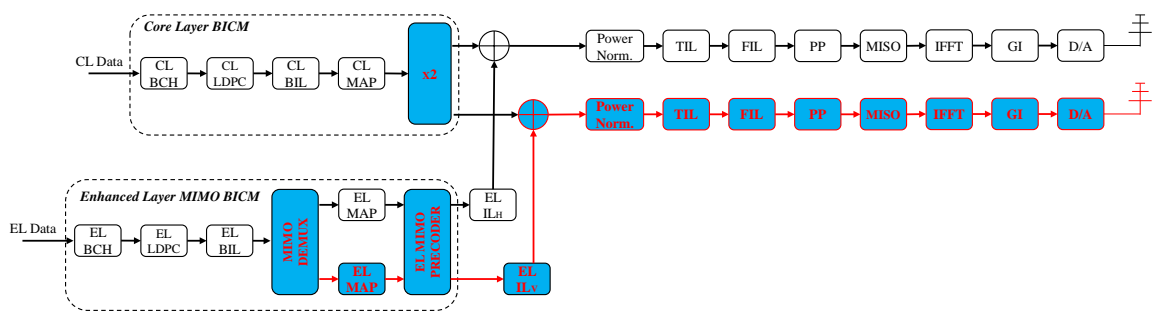

(a) CL MISO and EL MIMO transmitter configuration.

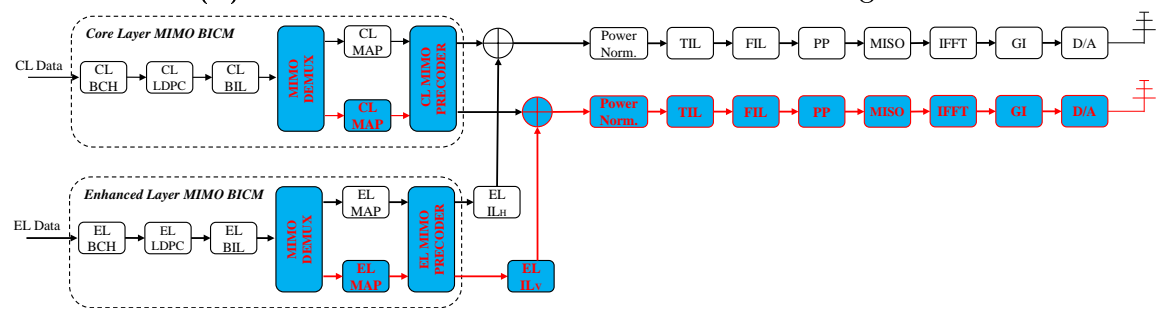

(b) CL MIMO and EL MIMO transmitter configuration.

Figure 7.3: LDM and MIMO transmitter block diagrams for the two joint transmissions considered during this thesis. Blue-coloured blocks represent additional complexity compared to a baseline LDM transmitter.

located antennas with cross-polar polarization for getting spatial multiplexing without a significant increase in the device size.

The potential performance benefits against additional complexity by employing a MIMO scheme in conjunction with LDM were investigated in Chapter 6 . Two combinations were extracted. On the one hand, with the objective of a low-complex mobile receiver, it was proposed to transmit the CL via a colocated MISO scheme, while the EL was transmitted with a MIMO scheme. Hence, CL could exploit the spatial diversity while the EL could increase the data rate by spatial multiplexing. It was justified since multiplexing gains are particularly noticeable at high SNR values [106], which does only apply to the EL. On the other hand, if there were no manufacturers' limitations on the mobile receivers implementation, the use of MIMO schemes for the two LDM layers was next proposed. It may increased not only the reliability of the CL but also the capacity. In addition, it was considered whether or not to enable TDCFS filtering. Figure 7.3 describes the two transmitter block diagrams when MIMO and LDM are jointly used. From the figure, the two proposals fluctuate on the CL BICM chain. Nevertheless this change modifies drastically the receiver implementations (see Section 7.2.9). 
Table 7.8: CL and EL SNR thresholds at BER $=10^{-4}$ for DVB-NGH mobile channel $\left(f_{D}=33.3 \mathrm{~Hz}\right)$ and MGM channel, respectively. NP and WH MP encodings are considered.

\begin{tabular}{cccccc}
\hline \hline & $\begin{array}{c}\text { MISO CL } \\
\text { MIMO EL } \\
\text { (wo TDCFS) }\end{array}$ & $\begin{array}{c}\text { MISO CL } \\
\text { (with TDCFS) }\end{array}$ & $\begin{array}{c}\text { MIMO CL } \\
\text { (wo TDCFS) }\end{array}$ & $\begin{array}{c}\text { MIMO CL } \\
\text { (with TDCFS) }\end{array}$ \\
\hline \multirow{2}{*}{$C L$} & \multirow{2}{*}{ (wP } & $15.0 \mathrm{~dB}$ & $3.0 \mathrm{~dB}$ & $3.7 \mathrm{~dB}$ & $3.7 \mathrm{~dB}$ \\
\cline { 2 - 6 } & $W H$ & $13.6 \mathrm{~dB}$ & $2.5 \mathrm{~dB}$ & $3.7 \mathrm{~dB}$ & $3.7 \mathrm{~dB}$ \\
\hline \multirow{2}{*}{$E L$} & $\mathrm{NP}$ & $27.8 \mathrm{~dB}$ & $27.2 \mathrm{~dB}$ & $27.4 \mathrm{~dB}$ & $27.4 \mathrm{~dB}$ \\
\cline { 2 - 6 } & $\mathrm{WH}$ & $28.3 \mathrm{~dB}$ & $27.2 \mathrm{~dB}$ & $27.8 \mathrm{~dB}$ & $27.8 \mathrm{~dB}$ \\
\hline \hline
\end{tabular}

Table 7.8 presents the SNR threshold obtained for the CL with the DVB-NGH mobile channel, and for the EL with the Modified Guilford Model (MGM) channel. These two MIMO channel models were extracted from field measurement campaigns [113], [114]. The two MP encodings, WH and NP were considered, but the MIMO precoder was disabled (i.e. plain precoder). In order to make a fair comparison in terms of spectral efficiency, the CL was transmitted $1 \times$ QPSK $4 / 15$ in the MISO use cases (first and second column), while $2 \times$ QPSK $2 / 15$ was transmitted in the MIMO use cases (third and fourth column).

It was observed that the use of a MISO scheme with TDCFS (second column) exploited more efficiently the spatial diversity than in MIMO schemes. It could also be seen that the performance of the EL did not vary due to the use of a MISO or a MIMO scheme on the CL. In addition, it was observed that NP outperforms WH about $0.5 \mathrm{~dB}$ in most of the cases, thanks to the $3 \mathrm{~dB}$ boosting pilot power.

Overall, the MISO TDCFS on the CL and MIMO on the EL was recommended for the joint co-located MIMO and LDM transmission. 


\subsection{Implementation Aspects}

This section examines the main implementation aspects that have been analyzed at receiver side when LDM is jointly used with other advanced technologies. Despite the LDM analysis can be split into two different receiver types, mobile and fixed receivers, the main constraints were common to both layers.

Figure 7.4, and Figure 7.5 depict the mobile and fixed receiver block diagrams assumed during this thesis, respectively. The blue-coloured blocks were the additional blocks needed for the joint transmission with MultiRF or with distributed MISO technologies.

In overall it was considered that the jointly use of LDM with the other advanced technologies did not require extra constraints, other than those related to each technology by itself. A summary of the different blocks in a receiver that have been analyzed during this thesis is presented next.

\subsubsection{Tuner}

The tuner was slightly analyzed in Section 4.4.1 for the joint LDM and TFS transmission. It was mentioned that in order to implement a TFS solution with only 1-tuner at receiver, time gaps had to be inserted between data slots. These time gaps should take into account not only tuning but also channel estimation operations for the time interpolation among SP at the start and end of each data slot. In addition, it was also highlighted that these time gaps restricted the peak service data rate up to $51 \%$ (Table 4.3 and 4.4).

\subsubsection{Channel Estimator}

The channel estimator implementation impacts significantly on the overall performance of a service. In OFDM receivers, the two-dimensional task of a channel estimator is commonly simplified into two simpler one-dimensional tasks. The first step is the extraction of the channel state at SP carriers. Different estimation techniques can be used, such as LS, Maximum Likelihood (ML), or Linear Minimum Mean Square Error (LMMSE) estimation. For channel estimation at SP, the simplest technique LS method has been assumed for this thesis.

The second step is the time and frequency interpolation between SP for getting the channel state at data carriers. The longer the interpolation method in number of taps, the more accurate the channel estimation. However, this is 


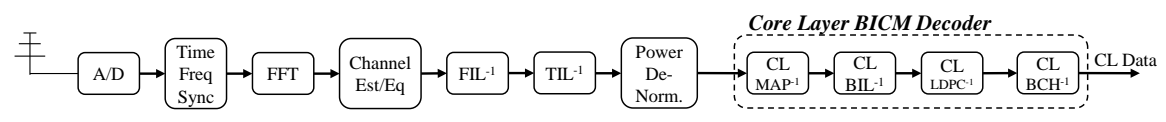

(a) Baseline mobile receiver block diagram.

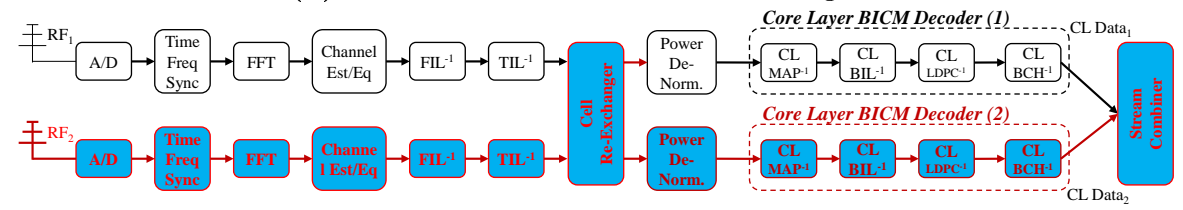

(b) Mobile receiver block diagram when CB is also employed.

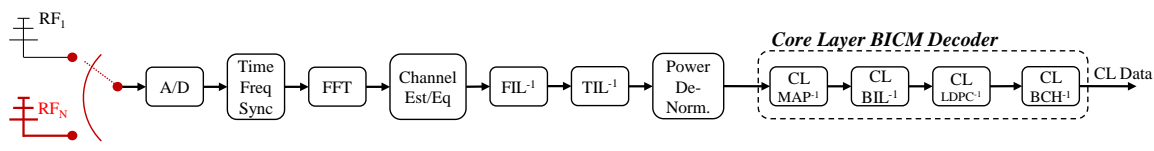

(c) Mobile receiver block diagram when TFS is also employed.

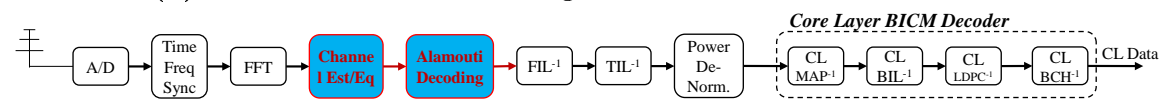

(d) Mobile receiver block diagram when distributed MISO Alamouti is also employed.

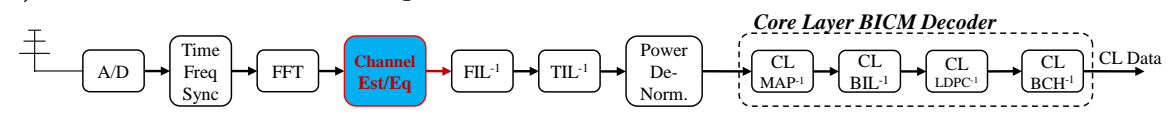

(e) Mobile receiver block diagram when co-located MISO is employed on the CL and MIMO on the EL.

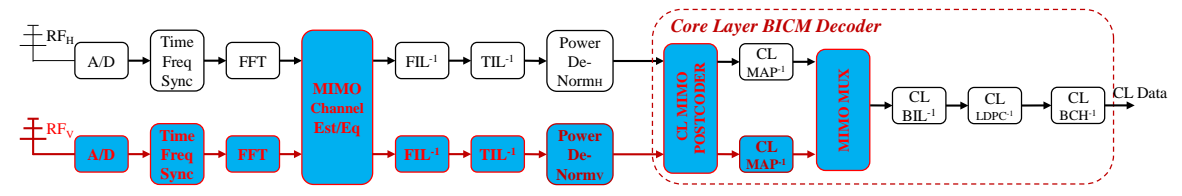

(f) Mobile receiver block diagram when co-located MIMO is employed on the CL and MIMO on the EL.

Figure 7.4: LDM mobile receiver block diagrams for the different joint transmissions considered during this thesis. Blue-coloured blocks represent additional complexity compared to a baseline LDM mobile receiver.

unattractive to manufacturers. In particular, the time interpolation is the element that generates a greater increase in receiver costs. The higher the number of OFDM symbols to be gathered for time interpolation, the greater the required memory for storing the signal stream before equalization. Whereas two different frequency interpolators have been used in this thesis (Wiener 
filtering in Chapter 3 and FFT in Chapter 5), the linear interpolation was assumed for time interpolation.

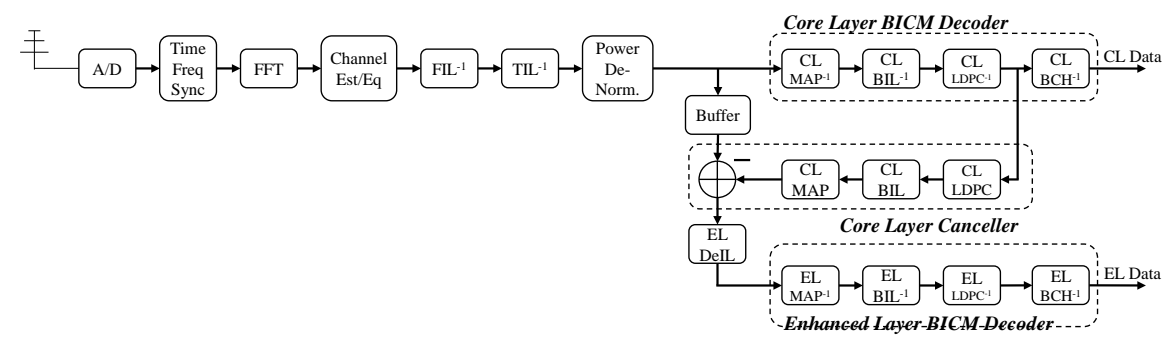

(a) Baseline fixed receiver block diagram.

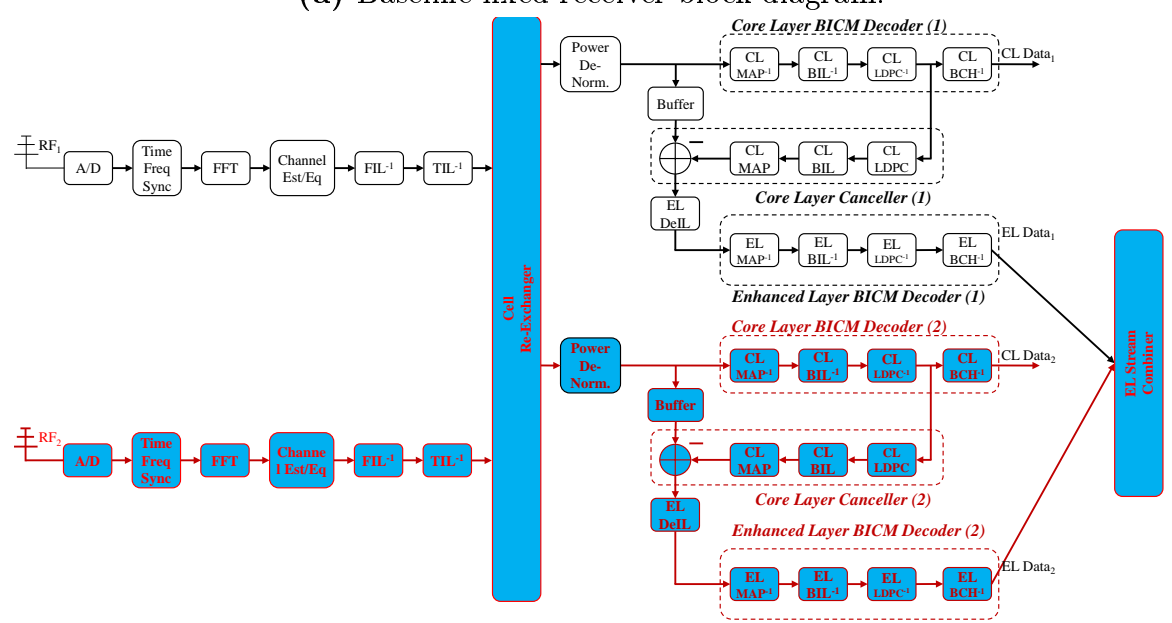

(b) Fixed receiver block diagram when $\mathrm{CB}$ is also employed.

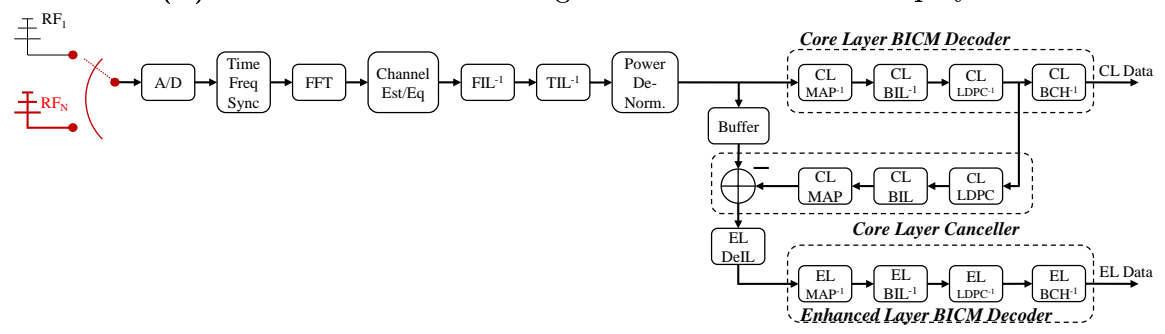

(c) Fixed receiver block diagram when TFS is also employed.

Figure 7.5: LDM fixed receiver block diagrams for the different joint transmissions considered during this thesis. Blue-coloured blocks represent additional complexity compared to a baseline LDM fixed receiver. 


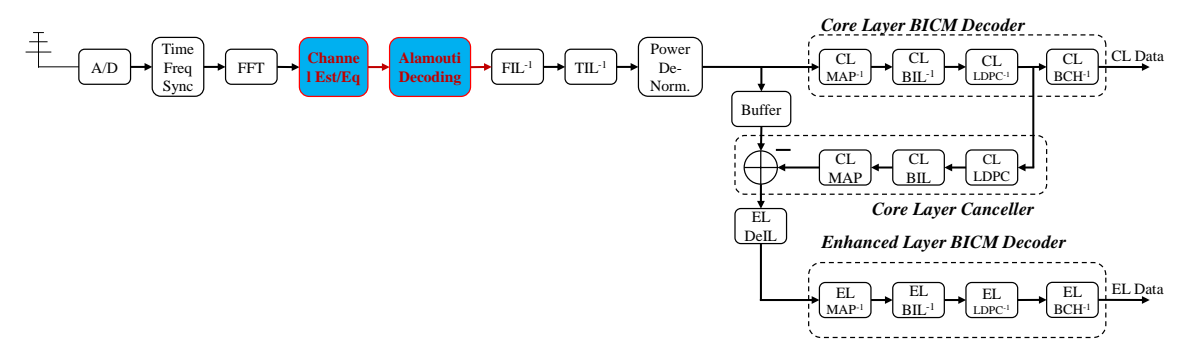

(d) Fixed receiver block diagram when Alamouti is also employed.

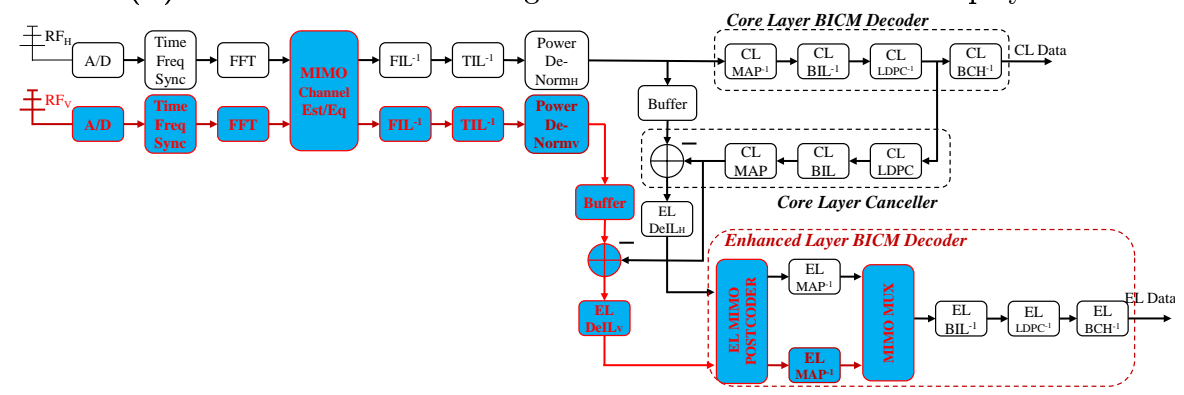

(e) Fixed receiver block diagram when co-located MISO is employed on the CL and MIMO on the EL.

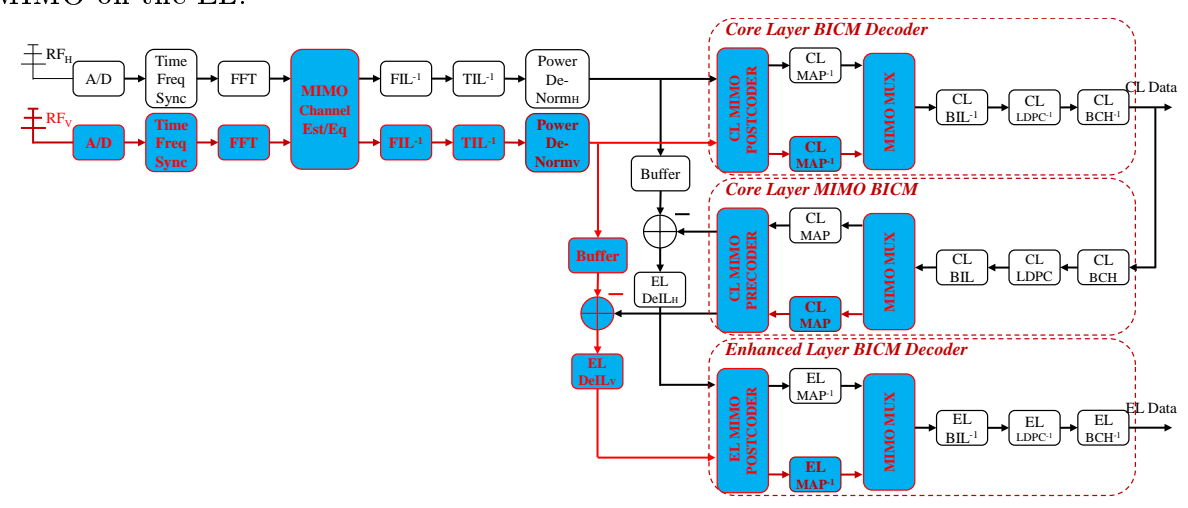

(f) Fixed receiver block diagram when co-located MIMO is employed on the CL and MIMO on the EL.

Figure 7.5: LDM fixed receiver block diagrams for the different joint transmissions considered during this thesis. Blue-coloured blocks represent additional complexity compared to a baseline LDM fixed receiver.

It should be noted that MP encodings were used for the MISO Alamouti scheme in Chapter 5. For the proper Alamouti decoding, these MP required a higher 
Table 7.9: Channel Estimator memory requirements (in subcarriers)

\begin{tabular}{ccc}
\hline \hline SISO & MISO WH & MISO NP \\
\hline 96775 & 110600 & 207375 \\
\hline \hline
\end{tabular}

memory increase compared to a baseline SISO channel estimator. Table 7.9 quantifies the number of subcarriers to be stored for the worst case with SISO and each MP encoding. As it can be seen, while WH requires a small increase with respect to SISO, NP encoding requires more than double. This value can be considered prohibitive to manufacturers.

\subsubsection{Equalizer}

The equalization process makes use of the channel components provided by the channel estimator in order to obtain the transmitted signal. It has been traditionally implemented with a simple linear type, such as Zero Forcing (ZF) or Minimum Mean Square Error (MMSE). Hence, the number of operations is $\mathcal{O}(n)$. However, Alamouti decoding requires the storage of two consecutive subcarriers from the two estimated channel components. Hence, for the proper equalization a pseudo-inverse matrix solution as it is defined in Section 5.3.2 is needed. This pseudo-matrix inversion increases the number of operations to $\mathcal{O}\left(n^{3}\right)$.

\subsubsection{Time De-Interleaver (TDIL)}

The TDIL has been traditionally considered as the most memory demanding receiver block by manufacturers. ATSC 3.0 specification has limited the TDIL memory to a maximum of $2^{19}=524288$ cells. Taking a look into the different implementations for mobile and fixed receivers (Figure 7.3 and Figure 7.4), only the joint transmission with $\mathrm{CB}$ implies an additional constraint. The 2 -tuner operation mode required of doubling the TDIL memory up to $2^{20}$ cells. 

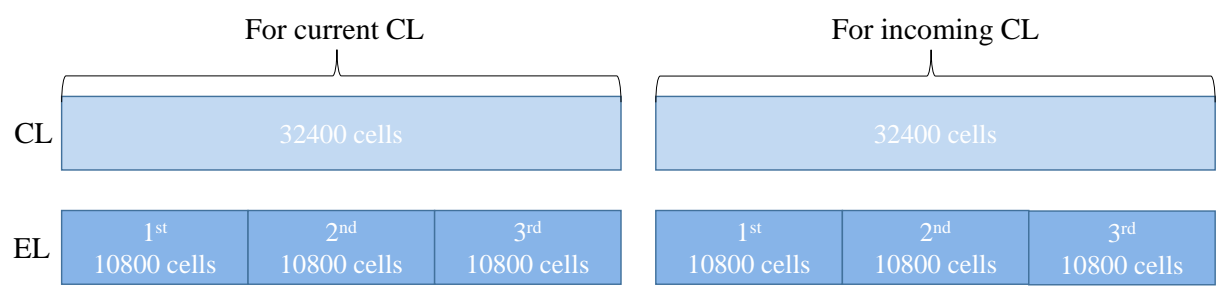

Figure 7.6: Maximum memory LDM buffer. One FEC CL 64800k codeword with QPSK constellation, and three FEC EL 64800k codewords with $64 \mathrm{NUC}$ constellation.

\subsubsection{Cell Re-Exchanger}

Inter-RF frequency interleaving is achieved in $\mathrm{CB}$ when it operates under the SNR Averaging mode. To ensure the even distribution of data across the two RF channels, an additional block called cell exchanger is employed. It distributes the odd and even cells of each FEC codeword in each RF channel respectively. At the receiver side, this exchange is undone with the cell reexchanger. Since the cell exchange is a linear process at cell level, it does not require special memory or latency attention.

\subsubsection{LDM Buffer}

An LDM buffer has to be implemented on fixed receivers for getting the EL. It should store some amount of the TDIL output cells awaiting for the CL cancellation. Its size highly depends on the constellation order and the FEC codeword length of the CL and EL. The straightforward implementation is to use two memory blocks in order to avoid memory conflicts between the current FEC codeword and the next one. Taking into account all the potential combinations, the maximum buffer size increases to 64080 cells, as it is depicted in Figure 7.6 [51]. From the different studies of this thesis, only the joint transmission with CB demanded a second LDM buffer for the second receiving chain. However, the maximum buffer size is still relatively small compared to the TDIL memory (64800 versus 524288 cells, about $12 \%$ ), so that it is not the most limiting element at receiver. Regarding latency requirements, the time needed for the CL demodulation and subsequent reconstruction should be faster than the TDIL read out rate ${ }^{1}$. Otherwise, the EL may not be correctly demodulated.

\footnotetext{
1 As a numerical example, the read out rate of DVB-T2 TDIL was established around $7.6 \cdot 10^{6}$ cells/s in [68].
} 
Table 7.10: Number of Euclidean distances of the different demapping approaches

\begin{tabular}{cccc}
\hline \hline \multicolumn{2}{c}{ Mobile Receiver } & \multicolumn{2}{c}{ Fixed Receiver } \\
$G D$ & $O D$ & $G D+H C$ & $O D+S C$ \\
\hline $2^{m_{C L}}$ & $2^{m_{C L}+m_{E L}}$ & $2^{m_{C L}}+2^{m_{E L}}$ & $2^{m_{C L}+m_{E L}+1}$ \\
\hline \hline
\end{tabular}

\subsubsection{Demapping}

Chapter 2 investigated different demapping approaches for increasing the CL and EL performance. Significant gains were achieved in particular scenarios. However, they can be considered out of the LDM scope. In addition, these gains were obtained at the expense of an increased complexity. Section 2.2.3 estimated the number of Euclidean distances to be computed by the GD $+\mathrm{OD}$, and $\mathrm{HC}+\mathrm{SC}$ approaches. They are presented in Table 7.10, where $m_{C L}$ and $m_{E L}$ represented the number of transmitted bits per symbol of the CL and EL, respectively. As it can be observed, the GD approach provides a low-complexity demapper implementation, which results in the most practical implementation for LDM systems.

\subsubsection{LDPC decoding}

In Section 7.2.6 it was mentioned that the CL cancellation processing should be performed faster that the TDIL read out rate in order not to overflown the LDM buffer. As the cancellation process requires of the previous CL decoding, the latency for the CL LDPC decoding should also be considered. Nevertheless, since the operational SNR of the EL is much more higher than the CL SNR threshold, the number of LDPC iterations for the CL decoding are drastically reduced [51].

\subsubsection{MIMO}

The additional complexity when MIMO Spatial Multiplexing (SM) mode of ATSC 3.0 is jointly used with LDM was analyzed in Section 6.4.3 of Chapter 6. Table 7.11 summarizes the complexity of each block for mobile and fixed receivers. As it can be observed, the complexity increase required of doubling every receiver block, but it came mainly from the use of a 2-tuner receiver of multiple antenna schemes rather than from LDM. Doubling the LDM buffers in MIMO fixed receivers is the only additional block required from LDM. How- 
Table 7.11: MIMO and LDM receivers complexity summary.

\begin{tabular}{ccc}
\hline \hline & $\begin{array}{c}\text { MIMO Mobile } \\
\text { receiver }\end{array}$ & $\begin{array}{c}\text { MIMO Fixed } \\
\text { receiver }\end{array}$ \\
\hline Channel Estimator & $114.3 \%(\mathrm{WH})$ & $228.6 \%(\mathrm{WH})$ \\
(\% memory increase) & $214.3 \%(\mathrm{NP})$ & $428.6 \%(\mathrm{NP})$ \\
\hline Equalizer & $\mathcal{O}\left(n^{3}\right)$ & $\mathcal{O}\left(n^{3}\right)$ \\
\hline Frequency De-Interleaver (FDIL) & $2 \times \mathrm{SISO}$ & $2 \times \mathrm{SISO}$ \\
\hline acTDIL & $2 \times \mathrm{SISO}$ & $2 \times \mathrm{SISO}$ \\
\hline LDM buffer & - & $2 \times \mathrm{SISO}$ \\
\hline BICM ${ }^{-1}$ & $2 \times \mathrm{SISO}$ & $2 \times \mathrm{SISO}$ \\
\hline \hline
\end{tabular}

ever, as it has been mentioned it constituted just a $12 \%$ compared to the TDIL memory.

In summary, as it has been discussing, the additional constraints of combining LDM with other advanced technologies can be considered admissible. As a matter of fact, the main demands, such as the 2-tuner receiving architecture or the Time De-Interleaver memory, were already adopted in the ATSC 3.0 standard. Given the performance increase of the combination of them, it is recommended to be included in future DTT standards. 



\section{Chapter 8}

\section{Conclusions and Future Work}

This dissertation has investigated advanced technologies to optimize the Layered Division Multiplexing (LDM) mode of the next-gen U.S. Digital Terrestrial Television (DTT) standard, ATSC - Third Generation (ATSC 3.0). The simultaneous delivery of TV services to mobile and fixed receivers is one of the main objectives of next-gen DTT systems. The next-gen European DTT standards, DVB - Terrestrial Second Generation (DVB-T2) and Digital Video Broadcasting - Next Generation Handheld (DVB-NGH) accomplish this objective by Time Division Multiplexing (TDM). ATSC 3.0 has also adopted the power division multiplexing mode, known as LDM. The LDM signal consists of the superposition of two independent services, namely layers, with different power levels. Therefore, while TDM or Frequency Division Multiplexing (FDM) distribute the orthogonal radio resources (time and frequency) among the multiplexed services, LDM layers use all the frequency and time resources but with different power levels, i.e. it is a Non-Orthogonal Multiplexing (NOM) solution. In the literature it has been extensively demonstrated that LDM exceeds TDM and FDM performance at the expense of more complex receivers [45]-[51]. Each LDM layer, defined as Core Layer (CL) and Enhanced Layer (EL) passes through different Bit-Interleaved Coded Modulation (BICM) chains, and are next superimposed with different power levels according to a configurable parameter, known as Injection Level $(\Delta)$. For simplicity, the LDM layers in ATSC 3.0 share the rest of physical layer 
blocks, including the Time Interleaver (TIL), the Pilot Pattern (PP), the Fast Fourier Transform (FFT) size, and the Guard Interval (GI). Depending on the intended layer, two receiver types are categorized. Straightforward mobile receivers, which are only intended to obtain the CL, regards the EL as an additional Additive White Gaussian Noise (AWGN). Therefore, there are no additional complexity constraints compared with traditional single layer receivers. By contrast, more complex fixed receivers, which are intended to obtain the EL, are required, as they have to perform a Successive Interference Cancellation (SIC) process.

The main investigation of this thesis can be divided into two parts. A first part aims to maximize the performance of the two LDM layers, by optimizing the ATSC 3.0 baseline transceiver chain. Two new demapping algorithms, one for each layer, have been introduced. In addition, the optimum pilot configuration, taking into account that both layers must share the same PP, has been also derived. The second part of the thesis investigates the potential conjunction of LDM with other disruptive technologies that has been adopted for next-gen DTT systems, such as Multi-Radio Frequency Channel (MultiRF) channel aggregation, distributed Multiple-Input Single-Output (MISO) schemes, or the co-located Multiple-Input Multiple-Output (MIMO) profile of ATSC 3.0. The main implications aspects at transmitter and receiver sides and the performance gains have been analysed. This concluding chapter extracts the main contributions of the thesis and proposes further research topics.

\subsection{Concluding Remarks}

\subsubsection{Baseline optimization}

\section{Demapping Optimization}

Two new demapping approaches for increasing the CL and EL performance have been proposed, at the expense of a higher complexity. Due to complexity issues, the CL demapping process has commonly assumed the EL as AWGN like interference. This assumption is not valid when the power of the LDM layers is similar and when the CL does not use a robust coding rate. The new CL demapping approach, which considers the knowledge of the EL constellation, brings performance gains up to $10 \mathrm{~dB}$ at low $\Delta$, and high CL coding rates. Nevertheless, the number of Euclidean distances to be computed is in turn increased. Whereas the commonly used demapping approach calculates 
$2^{m_{t}}$ Euclidean distances, the proposed approach requires $2^{m_{t}+m_{b}}$, where $m_{t}, m_{b}$ represents the number of transmitted bits per symbol of the CL and EL, respectively. Regarding the proposed EL demapping approach, it forwards the optimized Log-Likelihood Ratios (LLR) from the CL as a-priori information. Hence, it eliminates the traditional hard SIC process. Based on the previous scenario, performance gains in the range of $4 \mathrm{~dB}$ are accomplished for EL low coding rates. However, the number of Euclidean distances to be computed is again increased from $2^{m_{t}}+2^{m_{b}}$ to $2^{m_{t}+m_{b}+1}$. Other implementation aspects, such as the increased power consumption by LDPC decoders in low Signal-toNoise Ratio (SNR) conditions, should also be bear in mind.

\section{Pilot Optimization}

Whereas DVB-T2 permits 8 different Scattered Pilot (SP) patterns with a unique pilot amplitude, ATSC 3.0 offers a higher flexibility with up to 16 different SP patterns, where each one can use up to 5 different pilot amplitudes, called pilot boostings. Different trade-offs should be bear in mind. Regarding SP density, the selected SP pattern must be sufficiently dense to follow channel variations in time (Doppler tolerance) and frequency (echo tolerance) domain. However, the denser the SP, the lower the data rate. Considering that both LDM layers share the same pilot configuration, this pilot density (required by the CL) versus data rate (required by the EL) is further compromised. Regarding pilot boosting, the channel accuracy is increased by using higher values. However, the higher the pilot carriers amplitude, the lower the data carriers amplitude and, in turn, the lower the operational SNR. The studies have been done with different fading channels, DVB Fixed Rice (DVB-F1) and $0 \mathrm{~dB}$ echo (50\% GI) for fixed reception and Typical Urban (TU-6) for mobile reception. The results have been obtained not only for LDM but also for TDM so that it can be used as a pilot configuration guideline to ATSC 3.0 broadcasters.

In the case of LDM, since the CL employs very robust Modulation and Coding Rate (MODCOD), the channel estimation error is not relevant on the final performance. However, if the CL has not been properly obtained, a cancellation error is forwarded to the EL. This error is known as Cross-Layer Interference (CLI), which is proportional to the channel estimation error and the $\Delta$. From the simulation results, it has been observed that LDM mode requires a denser SP pattern than TDM. Nevertheless, it has been observed that the use of a dense SP patterns is not required, as all of them have been designed to cope with long echoes and high speeds. Therefore, SP12_2 has been recommended for TDM, while SP6_2 for LDM. Regarding pilot boosting, it has been found 
that the optimum pilot boosting depends on the receiver implementation. In particular, it mainly depends on the frequency interpolator used at the channel estimation process. Whereas the straightforward interpolators, such as linear interpolators are optimized for a medium pilot boosting value (pilot boosting 3 ), more sophisticated receivers, such as Wiener filtering [87] are intended to used the minimum pilot boosting (pilot boosting 0 ).

\subsubsection{Joint LDM and advanced technologies transmissions}

\section{LDM with MultiRF technologies}

The LDM combination with Channel Bonding (CB) has been evaluated. This combination is allowed in the ATSC 3.0 physical layer protocol. However, they had not been studied until now. In addition, LDM with the other MultiRF channel technology proposed in next-gen DTT standards, Time-Frequency Slicing (TFS), has been also considered. There are two main advantages that MultiRF technologies offer to a DTT transmission. On the one hand, a data rate increase, exceeding the net capacity of a single Radio Frequency (RF) channel (with Plain $C B$ mode). On the other hand, an RF performance increase by means of inter-RF interleaving (with $S N R$ Averaging $C B$ mode and TFS). Two use cases have been proposed. The most complete combination is the transmission of both LDM layers with SNR Averaging $C B$ mode. It allows doubling the service data rate, as well as improving transmission robustness of both LDM layers. However, this combination demand more complex mobile receivers. The second use case only applies the Plain CB mode on the EL, so that different CL streams are transmitted. Thus, mobile receiver implementations are still using only 1-tuner RF chain. Nevertheless, this second use case cannot exploit inter-RF frequency interleaving, but it can double EL data rate.

The performance results for the first use case (SNR Averaging $C B$ on both layers) show that high gains are obtained with uncorrelated channels for CL pedestrian reception $(2.1 \mathrm{~dB}$ for $\mathrm{TIL}=200 \mathrm{~ms})$. However, these gains depend on the time interleaving durations. The longer the duration, the lower the MultiRF gains. This is explained by the diminishing marginal returns of diversity concept [46]. In addition, important gains can be exploited from the SNR imbalances of the RF channels. These gains depends on the frequency separation among them (from $0.5 \mathrm{~dB}$ to $2.5 \mathrm{~dB}$ on $30 \mathrm{MHz}$ and $150 \mathrm{MHz}$ frequency separation, respectively). 


\section{LDM with distributed MISO schemes}

The joint transmission of LDM mode with the distributed MISO scheme of ATSC 3.0, Transmit Diversity Code Filter Sets (TDCFS), has also been studied. Again, although the combination is allowed in the specification, it has not been evaluated so far. TDCFS de-correlates the signals from the different transmitters to avoid destructive interferences. It has been concluded that the combination of LDM with the TDCFS does not require extra complexity constraints at receivers. In the performance simulations it has been found that no spatial diversity gains are provided in mobility conditions for the CL. It is also explained by the diminishing marginal returns of diversity concept. Negligible gains have also been obtained by using TDCFS on the EL.

In addition, the joint transmission of LDM with the distributed MISO scheme of DVB-T2, MISO Alamouti has also been investigated. Alamouti encoding achieves full diversity by sending the same but orthogonal signals between transmitters. In contrast with TDCFS, Alamouti requires of orthogonal MIMO Pilot (MP) patterns, which leads to more complex channel estimator and equalizer implementations. Regarding performance simulations, the two MP patterns of ATSC 3.0 have been evaluated, Null Pilot (NP), and WalshHadamard (WH). Compared to Single-Input Single-Output (SISO), NP halves the speed limit but maintains the echo tolerance, while WH echo tolerance is halved, keeping the speed limit. Alamouti gains of at least $1.8 \mathrm{~dB}$ have been achieved for the EL when the ATSC 3.0 MP NP encoding is used. If the MP $\mathrm{WH}$ encoding is used these gains are $0.5 \mathrm{~dB}$ smaller. Overall, although it requires of a more complex receiver, the distributed MISO Alamouti scheme has been proposed to be considered for future ATSC 3.0 releases.

\section{LDM with Co-Located MIMO Schemes}

The joint transmission of LDM with the co-located MIMO profile of ATSC 3.0 has been carried out last. Two main use cases have been proposed, according to the mobile receiver complexity. In the first use case, the CL is transmitted in a co-located MISO scheme, while the EL exploits the spatial multiplexing from MIMO. Therefore, mobile receivers are still implemented with a single antenna, and they slightly increase their complexity in terms of channel estimator memory requirements. In the second use case, both layers are broadcasted via a MIMO approach. This second use case will highly increase not only fixed receivers complexity, but also mobile receivers complexity as two antennas are demanded. This is, in turn, translated into higher device's cost. Special focus on the channel estimator's memory requirement has been analyzed. In com- 
Table 8.1: ATSC 3.0 FFT-GI-SP recommendation.

\begin{tabular}{ccc}
\hline \hline \multicolumn{3}{c}{ Pilot density } \\
\hline TDM mobile & TDM fixed & LDM \\
\hline FFT 8k & FFT 32k & FFT 16k \\
GI3_512 & GI3_512 & GI5_1024 \\
SP12_2 & SP12_2 & SP6_2 \\
\hline \hline
\end{tabular}

\begin{tabular}{ccc}
\hline \hline \multicolumn{3}{c}{ Pilot boosting } \\
\hline Linear & $F F T$ & Wiener \\
\hline 3 & 4 & $0-1$ \\
\hline \hline
\end{tabular}

parison with a baseline SISO receiver, the use of MP encodings increase the required channel estimator memory. The lowest memory increase is originated on mobile receivers of the first use case. When WH encoding is used, only a $14.3 \%$ compared to baseline SISO receivers is needed. At the other end, the receivers of second use case increase their memory requirements up to $428.6 \%$ when NP encoding is considered.

The performance of the CL and EL is evaluated with different channel models extracted from field measurement campaigns. Whereas the DVB-NGH MIMO channel is used for the CL performance on mobility conditions, the Modified Guilford Model (MGM) is considered for the EL performance at fixed roof-top reception. It is observed that spatial multiplexing gains at CL are insufficient for the additional complexity on mobile receivers. Furthermore, the use of MISO or MIMO over the CL does not affect the spatial multiplexing gains of the EL. In summary, taking into account the complexity and performance trade-off, the first use case is proposed for next-gen DTT standards, i.e. the use of MISO on the CL and MIMO on the EL. The two MP encodings are also compared. NP encoding outperforms $\mathrm{WH}$ about $0.5 \mathrm{~dB}$ on both layers. However, it is considered not enough compared with the memory increase requirements.

\subsection{Recommendations for Advanced LDM transmissions}

The investigations carried out during this thesis lead to a number of recommendations for the deployment of ATSC 3.0, and also for the design of future DTT systems. A balance between complexity increase and performance gains is assessed.

- The proposed demapping approaches, Optimum Demapping (OD) for the CL and Soft Cancellation (SC) for the EL, provide significant gains 
at unattractive ATSC 3.0 LDM operational SNR regions. Nevertheless, next-gen systems such as, Wideband reuse-1 (WiB) can benefit from high gains when e.g. the same signal is transmitted from multiple stations with small power imbalance among them [70], [116]. Moreover, as it is envisaged for Quadrature Phase-Shift Keying (QPSK) signals, the number of distances to be computed is moderately increased.

- ATSC 3.0 is designed to provide flexible operating modes for a variety of conditions while keeping an efficient use of the spectrum. This thesis recommends the following waveform parameter choices, so that broadcasters can optimally deliver their intended services. SP12_2 is proposed as the optimum pilot configuration in TDM systems. The overhead is only $4.2 \%$, and it allows up to $105 \mathrm{~km}$ distance between Single Frequency Networks (SFN) transmitters for 32k FFT size (GI3_512), as well as speeds up to $350 \mathrm{~km} / \mathrm{h}$ in the Ultra-High Frequency (UFF) band for an $8 \mathrm{k}$ FFT size (GI3_512). For LDM systems, SP6_2 is considered as an optimum SP. The overhead is only $8.4 \%$, and considering a common $16 \mathrm{k}$ FFT size (GI5_1024), it allows up to $45 \mathrm{~km}$ distance between SFN transmitters for the EL, as well as speeds up to $180 \mathrm{~km} / \mathrm{h}$ in the UHF band for the CL. Regarding pilot boosting, a medium pilot boosting value is recommended for simple linear interpolators, the maximum value for widely used FFT interpolators, and the minimum value for sophisticated receivers, such as Wiener filtering. Table 8.1 presents the optimum FFT-GI-SP configuration for different transmitter and receiver implementations. These configurations have been included in [117].

- Aside from doubling the service data rate, the joint transmission of $\mathrm{CB}$ and LDM is particularly recommended for the EL performance, as limited performance gains for the CL are obtained due to the diminishing marginal returns of diversity.

- The introduction of distributed MISO schemes is also recommended for increasing the EL performance in fixed-rooftop conditions, where the TIL is not providing performance gains. Since TDCFS gains are only achieved at very limited SFN scenarios, MISO Alamouti scheme adopted in DVB-T2 rewards higher profits, at the expense of more complex receivers. Therefore, its inclusion in further ATSC 3.0 releases is highly recommended.

- Taking into account the complexity and performance trade-off, MISO on the CL and MIMO on the EL is proposed for next-gen DTT standards. It will not force to very complex mobile receivers, and EL can double the 
service data rate by spatial multiplexing. In addition, WH MP encoding is recommended despite the $0.5 \mathrm{~dB}$ performance loss versus NP.

\subsection{Future Research Topics}

\subsubsection{Further studies of LDM with co-located MIMO schemes}

All results obtained in Chapter 7 assumed a plain MIMO precoder, i.e. the three stages (Stream Combining, IQ Polarization Interleaving, and Phase Hopping) were disabled during the transmission. Hence, their impact is still missing. Taking a look at Figure 6.4, a different MIMO precoder per LDM layer was proposed for the second joint use case, but there are no results that demonstrates the suitability of this implementation. At first, this implementation offers a higher flexibility for each layer, optimizing their performance independently. For example, as the MODCOD of the CL and EL are initially different, the phase rotation angle of the Stream Combining stage should also be different. However, this implementation will require a more complex LDM cancellation process, which can be for example, extremely ambitious for the non-linear process of the IQ Polarization Interleaving stage.

In addition, same $\Delta_{H}=\Delta_{V}$ values have been assumed on each transmitter chain for simplicity. Nevertheless, different values can be assigned. This configuration can be satisfactory for those scenarios where one of the transmitting polarizations suffers of deep fading. Moreover, these scenarios can be used as a transition from SISO to MIMO, as it was recommended for DVB-NGH deployments, where the precoder reduces performance losses of uneven power allocation [108]. The introduction of asymmetrical constellations can also further reduce this performance loss by applying a more robust constellation to the antenna with less transmitting power.

\subsubsection{N-LDM and LDM Segmentation}

The LDM capabilities for the two layers case have been widely studied in the literature. However, LDM can be implemented with more layers. An information-theoretic analysis for the $N$-layer case has been introduced in [118], as well as initial performance simulations for 3-layer case in [71], [118]. Nevertheless, laboratory results as well as field trials should be further evaluated.

In another vein, although the use of LDM is complementary to other multiplexing modes, it has not been studied in detail. For example, the combination 
of LDM with TDM in ATSC 3.0 has been assessed in [64], [65]. It focuses on flexible framing with multiple Physical Layer Pipes (PLP) and LDM. Performance studies are provided by using different framing configurations consisting of three PLPs. The use of two CL PLPs superimposed onto an EL PLP is the TDM/LDM approach recommended. The joint use of LDM with FDM is being investigated for the next-generation terrestrial broadcasting standard in Japan [119], [120]. An improve frequency utilization and performance of the UHDTV service is proposed by applying LDM over the central segments for partial reception. As a result, the SNR threshold of the mobile stream is increased around $1.5 \mathrm{~dB}$ but the transmission rate of the UHDTV stream is increased since all segments are used. More detailed performance analysis under different channel conditions are still needed.

\subsubsection{SIC processing for Wideband Broadcasting}

DTT networks are traditionally planned assuming a given frequency reuse factor. With frequency reuse, the available RF channels are orthogonally distributed across the transmitting stations of the network, limiting the available spectrum per transmitter (typically 5-6 RF channels). To compensate this spectrum scarcity, high-capacity transmission modes are needed, such as the widely 256QAM $2 / 3$ mode used in DVB-T2 networks. These modes require high SNR thresholds, which, in turn, lead to high power transmissions. Wideband Broadcasting breaks with this frequency reuse concept, allowing all transmitters to potentially use all RF channels, by using an ultra-robust transmission mode (e.g. QPSK 1/2). The combination of wideband and frequency reuse 1 may result in more energy efficient networks, as the same capacity is achieved by extending the bandwidth rather than the transmit power.

It should be noted that decreasing frequency reuse down to 1 results in severe co-channel interferences, which may not only be resolved with the ultra-robust transmission mode. To improve the coverage performance, SIC processes can be implemented at receivers at the expense of higher complexity [116]. This situation resembles the initial Cloud Transmission concept, in which two signals from different transmitters were combined [49]. However, while in LDM several signals are aggregated at the modulation level, so that they are acquired at receiver as a single signal, in the Wideband system, single-layer signals are transmitted from different stations, so that they are not necessarily synchronized. Mechanisms that guarantee the proper signal acquisition and synchronization should be investigated. In addition, the number of received signals as well as the power level between them is at first unknown. Therefore, the SIC processes to be performed must be designed accordingly. 


\subsubsection{NOM for 5th Generation (5G) Mobile Systems}

Nowadays, the state-of-the-art DTT standard is ATSC 3.0. However, the Third Generation Partnership Project (3GPP) standardization forum is also enhancing the use of Point-to-Multipoint (PTM) technologies in mobile networks since Release 9 (Rel' 9) [121]. In fact, the development of $5 \mathrm{G}$ is considered as a good opportunity for a converged broadband and broadcast system [122].

NOM techniques, like LDM, are being considered in order to achieve higher spectrum efficiency in 5G cellular systems [44], [123]. Two Study Item (SI) have been developed inside 3GPP: 36.859 Study on Downlink Multi-User Superposition Transmission (MUST) for Rel-13 analyzed a Downlink NOM mode for Long Term Evolution (LTE)-Advanced Pro [52], and 38.812 Study on NonOrthogonal Multiple Access (NOMA) for NR, focused on an Uplink NOM, for 5G-New Radio was approved in March 2017, but delayed to focus on the 5G main standard (Rel' 15). The potential application of LDM in the 5G PTM transmission is explored in [124], [125]. It is highlighted that LDM gains will become higher thanks to the multiple antenna technologies adopted in 5G.

Multiple scenarios where LDM can enhance the current $4 \mathrm{G}$ performance can be considered. On the one hand, in $4 \mathrm{G}$ the broadcast services (evolved Multimedia Broadcast/Multicast Service (eMBMS)) are delivered in TDM mode with unicast. With LDM, the 5G PTM service could be delivered in the CL, while multiple unicast services can be delivered in the EL. Results in [124], [125] shows that LDM achieves higher spectrum efficiency than current TDM mode. On the other hand, if a stand-alone broadcast network is allowed by 5G, LDM can address the simultaneous broadcast delivery more efficiently than TDM.

Since Rel'15 is already published (December 2017), it is expected that the NOM SI will be shortly resumed. Thus, enhanced NOM variants will be proposed and should be evaluated in the 5G-NR framework [126]. 


\section{Bibliography}

[1] A/53 - ATSC Digital Television Standard, ATSC, 2007 (cit. on p. 1).

[2] Transmission System for Digital Terrestrial Television Broadcasting, ARIB, 2014 (cit. on p. 2).

[3] Framing Structure, Channel Coding and Modulation for Digital Television Terrestrial Broadcasting System, Chinese National Standard, 2006 (cit. on p. 2).

[4] Digital Video Broadcasting (DVB); Frame Structure Channel Coding and Modulation for Digital Terrestrial Television, ETSI, 1997 (cit. on p. 2).

[5] W. Y. Zou and Y. Wu, "COFDM: An Overview", IEEE Trans. on Broadcast., vol. 41, no. 1, pp. 1-8, 1995 (cit. on p. 2).

[6] Y. Wu, E. Pliszka, B. Caron, P. Bouchard, and G. Chouinard, "Comparison of Terrestrial DTV Transmission Systems: the ATSC 8-VSB, the DVB-T COFDM, and the ISDB-T BST-OFDM", IEEE Transact. on Broadcast., vol. 46, no. 2, pp. 101-113, 2000 (cit. on p. 2).

[7] M. Simon and M. A. Aitken, "An Overview of the North American ATSC M/H Mobile Broadcasting System and Its Next Generation ATSC 3.0", in Next Generation Mobile Broadcasting, D Gomez- 
Barquero, Ed., Boca Raton, FL, USA: CRC Press, 2013, pp. 91-122 (cit. on p. 2).

[8] C. E. Shannon, "A Mathematical Theory of Communication", Bell System Technical Journal, vol. 27, pp. 379-423, 623-656, 1948 (cit. on pp. 3, 11).

[9] "Digital Dividend - Insights for Spectrum Decisions", ITU, Tech. Rep., 2012 (cit. on p. 3).

[10] "The Future of Terrestrial Broadcasting", European Broadcasting Union, Tech. Rep. 013, 2011 (cit. on p. 3).

[11] "Final Acts WRC-07", ITU, Geneva, Switzerland, Tech. Rep., 2007 (cit. on p. 3).

[12] "Final Acts WRC-15", ITU, Geneva, Switzerland, Tech. Rep., 2015 (cit. on p. 3).

[13] D. Gomez-Barquero and M. W. Caldwell, "Broadcast Television Spectrum Incentive Auctions in the U.S.: Trends, Challenges and Opportunities", IEEE Communications Magazine, vol. 53, no. 7, pp. 50-56, 2015 (cit. on pp. 3,78 ).

[14] G. J. Sullivan, J. R. Ohm, W. J. Han, and T. Wiegand, "Overview of the High Efficiency Video Coding (HEVC) Standard", IEEE Trans. on Circuits and Systems for Video Technology, vol. 22, no. 12, pp. 16491668, 2012 (cit. on p. 3).

[15] T. K. Tan et al., "Video Quality Evaluation Methodology and Verification Testing of HEVC Compression Performance", IEEE Trans. on Circuits and Systems for Video Technology, vol. 26, no. 1, pp. 76-90, 2016 (cit. on p. 3).

[16] Digital Video Broadcasting (DVB); Frame Structure Channel Coding and Modulation for a Second Generation Digital Terrestrial Television Broadcasting System (DVB-T2), ETSI, 2011 (cit. on pp. 4, 78). 
[17] ATSC Standard - Physical Layer Protocol, ATSC, 2017 (cit. on pp. 4, $102,131)$.

[18] S. Saito et al., "8K Terrestrial Transmission Field Tests Using DualPolarized MIMO and Higher-Order Modulation OFDM", IEEE Trans. Broadcast., vol. 62, no. 1, pp. 306-315, 2016 (cit. on p. 4).

[19] A. Sato, T. Shitomi, T. Takeuchi, M. Okano, and K. Tsuchida, "Transmission Performance Evaluation of LDPC coded OFDM over Actual Propagation Channels in Urban Area. Examination for Next-Generation ISDB-T", in 2017 IEEE International Symposium on Broadband Multimedia Systems and Broadcasting (BMSB), Cagliari, Italy, 2017, pp. 1-5 (cit. on p. 4).

[20] Commercial Requirements for DVB-T2, Digital Video Broadcasting, 2007 (cit. on p. 4).

[21] R. Gallager, "Low-Density Parity-Check Codes", IRE Trans. on Information Theory, vol. 8, no. 1, pp. 21-28, 1962 (cit. on p. 4).

[22] D. Gozalvez, D. Gomez-Barquero, D. Vargas, and N. Cardona, "Time Diversity in Mobile DVB-T2 Systems", IEEE Trans. Broadcast., vol. 57, no. 3, pp. 617-628, 2011 (cit. on pp. 4, 85).

[23] D. Gozalvez, "Combined Time, Frequency and Space Diversity in Multimedia Mobile Broadcasting Systems", PhD thesis, Universitat Politecnica de Valencia, 2012 (cit. on p. 4).

[24] S. M. Alamouti, "A Simple Transmit Diversity Technique for Wireless Communications", IEEE Journal on Selected Areas in Commun., vol. 16, no. 8, pp. 1451-1458, 1998 (cit. on pp. 4, 103).

[25] I. Eizmendi et al., "DVB-T2: The Second Generation of Terrestrial Digital Video Broadcasting System", IEEE Trans. Broadcast., vol. 60, no. 2, pp. 258-271, 2014 (cit. on pp. 5, 50).

[26] L Fay, L Michael, D. Gomez-Barquero, N. Ammar, and M. W. Caldwell, "An Overview of the ATSC 3.0 Physical Layer Specification", IEEE 
Trans. Broadcast., vol. 62, no. 1, pp. 159-171, 2016 (cit. on pp. 5, 6, 26, $50,52,78,101,124)$.

[27] "Report and Order Authorizing Permissive Use of the "Next Generation" Broadcast Television Standard", Federal Communications Comssion, Tech. Rep. FCC 17-158, 2017 (cit. on p. 5).

[28] N. S. Loghin et al., "Non-Uniform Constellations for ATSC 3.0", IEEE Trans. on Broadcast., vol. 62, no. 1, pp. 197-203, 2016 (cit. on pp. 5, $50)$.

[29] M. Fuentes, "Non-Uniform Constellations for Next-Generation Digital Terrestrial Systems", PhD thesis, Universitat Politecnica de Valencia, 2017 (cit. on p. 5).

[30] P. Klenner et al., "Physical Layer Time Interleaver for the ATSC 3.0 System", IEEE Trans. Broadcast., vol. 62, no. 1, pp. 253-262, 2016 (cit. on pp. $5,52,87,128,156)$.

[31] S. LoPresto, R. Citta, D. Vargas, and D. Gomez-Barquero, "Transmit Diversity Code Filter Sets (TDCFSs), an MISO Antenna Frequency Predistortion Scheme for ATSC 3.0", IEEE Trans. Broadcast., vol. 62, no. 1, pp. 271-280, 2016 (cit. on pp. 5, 101, 102, 109, 126).

[32] L. Stadelmeier, D. Schneider, J. Zolner, and J. J. Gimenez, "Channel Bonding for ATSC 3.0", IEEE Trans. Broadcast., vol. 62, no. 1, 2016 (cit. on pp. 5, 78, 82, 101).

[33] J. J. Gimenez, "Improved Spectrum Usage with Multi-RF Channel Aggregation Technologies for the Next-Generation Terrestrial Broadcasting", PhD thesis, Universitat Politecnica de Valencia, 2015 (cit. on pp. 5, $7,78)$.

[34] D. Gomez-Barquero et al., "MIMO for ATSC 3.0", IEEE Trans. Broadcast., vol. 62 , no. 1, pp. 298-305, 2016 (cit. on pp. 5, 19, 124).

[35] D. Vargas, "Transmit and Receive Signal Processing for MIMO Terrestrial Broadcast Systems", PhD thesis, Universitat Politecnica de Valencia, 2016 (cit. on pp. 5, 124). 
[36] J. Montalban, "Solutions for New Terrestrial Broadcasting Systems Offering Simultaneously Stationary and Mobile Services", PhD thesis, Universidad del País Vasco / Euskal Herriko Unibertsitatea, 2014 (cit. on pp. $6,8,78)$.

[37] C. Regueiro, "Reception Performance Studies for the Evaluation and Improvement of the New Generation Terrestrial Television Systems", PhD thesis, Universidad del País Vasco / Euskal Herriko Unibertsitatea, 2017 (cit. on pp. 6, 27, 30).

[38] Benefits of Using Multiple PLP in DVB-T2, White Paper, Enensys Technologies, 2012 (cit. on p. 7).

[39] D. Gozalvez, D. Gomez-Barquero, I. Eizmendi, G. Berjon-Eriz, and M. Velez, "DVB-T2 for Mobile and Mobile DVB-T2 (T2-Lite)", in Next Generation Mobile Broadcasting, D Gomez-Barquero, Ed., Boca Raton, FL, USA: CRC Press, 2013, pp. 151-183 (cit. on pp. 7, 78).

[40] Digital Video Broadcasting (DVB); Next Generation Broadcasting System to Handheld - Physical Layer Specification (DVB-NGH), DVB, 2012 (cit. on p. 7).

[41] C. Hellge, T. Wiegand, E. G. Torre, D. Gomez-Barquero, and T. Schierl, "Efficient HDTV and 3DTV Services over DVB-T2 using Multiple PLPs with Layered Media", IEEE Comm. Magazine, vol. 51, no. 10, pp. 7682, 2013 (cit. on p. 7).

[42] S. Moon et al., "Enhanced Spatial Multiplexing for Rate-2 MIMO of DVB-NGH System", in 2012 International Conference on Telecommunications (ICT), Jounieh, Lebanon, Apr. 2012, pp. 1-5 (cit. on p. 7).

[43] M. Takada et al., "An Overview of the ISDB-T One-Seg Broadcasting, ISDB-Tsb and ISDB-Tmm", in Next Generation Mobile Broadcasting, D Gomez-Barquero, Ed., Boca Raton, FL, USA: CRC Press, 2013, pp. 151-183 (cit. on pp. 7, 78).

[44] L. Dai et al., "Non-Orthogonal Multiple Access for 5G: Solutions, Challenges, Opportunities, and Future Research Trends", IEEE Communications Magazine, vol. 53, no. 9, pp. 74-81, 2015 (cit. on pp. 7, 101, 124, 186). 
[45] L. Zhang et al., "Layered Division Multiplexing: Theory and Practice", IEEE Trans. Broadcast., vol. 62, no. 1, pp. 216-232, 2016 (cit. on pp. 7, $27,50,61,78,101,124,154,177)$.

[46] D. Tse and P. Viswanath, Fundamentals of Wireless Communication. New York, NY, USA: Cambridge University Press, 2005, ISBN: 0-52184527-0 (cit. on pp. 8, 116, 124, 133, 158, 177, 180).

[47] T. Cover, "Broadcast channels", IEEE Trans. on Information Theory, vol. 18 , no. 1, pp. 2-14, 1972 (cit. on pp. 8, 11, 12, 177).

[48] P. P. Bergmans and T. M. Cover, "Cooperative Broadcasting", IEEE Trans. on Information Theory, vol. 20, no. 3, pp. 317-324, 1974 (cit. on pp. $8,11,177)$.

[49] Y. Wu, B. Rong, K. Salehian, and G. Gagnon, "Cloud Transmission: A New Spectrum-Reuse Friendly Digital Terrestrial Broadcasting Transmission System", IEEE Trans. Broadcast., vol. 58, no. 3, pp. 329-337, 2012 (cit. on pp. 8, 12, 13, 26, 79, 177, 185).

[50] D. Gomez-Barquero and O. Simeone, "LDM Versus FDM/TDM for Unequal Error Protection in Terrestrial Broadcasting Systems: An Information-Theoretic View", IEEE Trans. Broadcast., vol. 61, no. 4, pp. 571-579, 2015 (cit. on pp. 8, 78, 101, 124, 177).

[51] S. I. Park et al., "Low Complexity Layered Division Multiplexing System for ATSC 3.0", IEEE Trans. Broadcast., vol. 62, no. 1, pp. 233-243, 2016 (cit. on pp. 8, 14, 15, 17, 28, 31, 51, 78, 101, 124, 139, 173, 174, 177).

[52] H. Lee, S. Kim, and J. H. Lim, "Multiuser Superposition Transmission (MUST) for LTE-A systems", in 2016 IEEE International Conference on Communications (ICC), 2016, pp. 1-6 (cit. on pp. 8, 186).

[53] S. Gadkari and K. Rose, "Time-Division Versus Superposition Coded Modulation Schemes for Unequal Error Protection", IEEE Trans. Communications, vol. 47, no. 3, pp. 370-379, 1999 (cit. on pp. 12, 27). 
[54] B. Rong et al., "Signal Cancellation Techniques for RF Watermark Detection in an ATSC Mobile DTV System", IEEE Trans. on Vehicular Technology, vol. 60, no. 8, pp. 4070-4076, 2011 (cit. on p. 13).

[55] L Michael and D. Gomez-Barquero, "Bit-Interleaved Coding and Modulation (BICM) for ATSC 3.0", IEEE Trans. Broadcast., vol. 62, no. 1, pp. 181-188, 2016 (cit. on pp. 15, 38, 50, 81, 127, 154).

[56] J. Montalban, M. Velez, I. Angulo, P. Angueira, and Y. Wu, "Large Size FFTs over Time-Varying Channels", Electronics Letters, vol. 50, no. 15, pp. 1102-1103, 2014 (cit. on pp. 15, 17, 51).

[57] L. Zhang et al., "Performance Characterization and Optimization of Mobile Service Delivery in LDM-Based Next Generation DTV Systems", IEEE Trans. Broadcast., vol. 61, no. 4, pp. 557-570, 2015 (cit. on p. 15).

[58] J. Montalban et al., "Performance Study of Layered Division Multiplexing Based on SDR Platform", IEEE Trans. Broadcast., vol. 61, no. 3, pp. 436-444, 2015 (cit. on pp. 15, 78).

[59] C. Regueiro et al., "LDM Core Services: Indoor and Mobile Performance in ATSC 3.0", IEEE Trans. Broadcast., vol. 62, no. 1, pp. 244-252, 2016 (cit. on pp. $15,17,51,78$ ).

[60] B. Lim et al., "Mobile Field Comparison Test of LDM and TDM Based on ATSC 3.0", in 2017 IEEE International Symposium on Broadband Multimedia Systems and Broadcasting (BMSB), Cagliari, Italy, 2017 (cit. on p. 16).

[61] S. I. Park et al., "Field Test Results of Layered Division Multiplexing for the Next Generation DTV System", IEEE Trans. Broadcast., vol. 63, no. 1, pp. 260-266, 2017 (cit. on p. 16).

[62] S. Park et al., "Field Comparison Tests of LDM and TDM in ATSC 3.0", IEEE Trans. Broadcast., vol. 64, no. 2, pp. 1-11, 2018 (cit. on p. 16).

[63] "High-VHF Field Measurements of ATSC 3.0 Digital Television Signals in Cleveland, Ohio", FCC, Tech. Rep., 2016 (cit. on p. 16). 
[64] J. Y. Lee et al., "Multiple Service Configurations Based on Layered Division Multiplexing", IEEE Trans. Broadcast., vol. 63, no. 1, pp. $267-$ 274, 2017 (cit. on pp. 16, 185).

[65] J. Lee et al., "Framing and Multiple-PLP Structures for LDM-based Next Generation Terrestrial Broadcasting Systems", in 2015 IEEE International Symposium on Broadband Multimedia Systems and Broadcasting (BMSB), Ghent, Belgium, 2015 (cit. on pp. 16, 185).

[66] W. Li et al., "Using LDM to Achieve Seamless Local Service Insertion and Local Program Coverage in SFN Environment", IEEE Trans. Broadcast., vol. 63, no. 1, pp. 250-259, 2017 (cit. on p. 16).

[67] J. Y. Lee et al., "Efficient Transmission of Multiple Broadcasting Services Using LDM and SHVC", IEEE Trans. Broadcast., vol. 64, no. 2, pp. 1-11, 2018 (cit. on p. 16).

[68] Implementation Guidelines for a Second Generation Digital Terrestrial Television broadcasting System (DVB-T2), ETSI, 2012 (cit. on pp. 17, $126,173)$.

[69] J. Morgade et al., "SFN-SISO and SFN-MISO Gain Performance Analysis for DVB-T2 Network Planning", IEEE Trans. Broadcast., vol. 60, no. 2, pp. 272-286, 2014 (cit. on pp. 19, 101).

[70] E. Stare, J. J. Gimenez, and P. Klenner, "WiB - A New System Concept for Digital Terrestrial Television (DTT)", The Best of IET and IBC 2016-2017, vol. 8, no. 1, pp. 4-9, 2016 (cit. on pp. 27, 41, 183).

[71] C. Regueiro et al., "LLR Reliability Improvement for Multilayer Signals", IEEE Trans. Broadcast., vol. 63, no. 1, pp. 275-281, 2017 (cit. on pp. $27,30,37,40,46,184)$.

[72] G. Caire, G. Taricco, and E. Biglieri, "Bit-Interleaved Coded Modulation", IEEE Trans. Information Theory, vol. 44, no. 3, pp. 927-946, 1998 (cit. on p. 29).

[73] S. Ten Brink, and J. Speidel, and Ran-Hong Yan, "Iterative Demapping and Decoding for Multilevel Modulation", in 1998 IEEE Global 
Telecommunications Conference (GLOBECOM '98), Sydney, Australia, 1998 (cit. on p. 30).

[74] C. Regueiro et al., "Improving LDPC Decoding Performance for ATSC 3.0 LDM Profiles", in 2017 IEEE International Symposium on Broadband Multimedia Systems and Broadcasting (BMSB), Cagliari, Italy, 2017 (cit. on pp. 32, 38).

[75] R. G. Gallager, Information Theory and Reliable Communication. Wiley, 1968 (cit. on p. 32).

[76] A. Martinez, A. G. i Fabregas, G. Caire, and F. Willems, "BitInterleaved Coded Modulation Revisited. A Mismatched Decoding Perspective", in 2008 IEEE International Symposium on Information Theory, 2008, pp. $2337-2341$ (cit. on pp. 33, 37).

[77] T. T. Nguyen and L. Lampe, "Bit-Interleaved Coded Modulation with Mismatched Decoding Metrics", IEEE Trans. Comm., vol. 59, no. 2, pp. 437-447, 2011 (cit. on p. 35).

[78] A. Alvarado, L. Szczecinski, R. Feick, and L. Ahumada, "Distribution of L-values in Gray-Mapped $M^{2}$-QAM: Closed-Form Approximations and Applications", IEEE Trans. Comm., vol. 57, no. 7, pp. 2071-2079, 2009 (cit. on pp. 36,37 ).

[79] K. J. Kim et al., "Low-Density Parity-Check Codes for ATSC 3.0", IEEE Trans. Broadcast., vol. 62, no. 1, pp. 189-196, 2016 (cit. on pp. 50, 80).

[80] E. Garro, J. J. Gimenez, D. Gomez-Barquero, and S. I. Park, "Pilot optimization for mobile services in atsc 3.0", in 2016 IEEE International Symposium on Broadband Multimedia Systems and Broadcasting (BMSB), 2016, pp. 1-5 (cit. on p. 51).

[81] P. F. Gomez, D. Gomez-Barquero, D. Gozalvez, A. Añorga, and M. Breiling, "Time Interleaving in DVB-NHG", in Next Generation Mobile Broadcasting, D Gomez-Barquero, Ed., Boca Raton, FL, USA: CRC Press, 2013, pp. 355-388 (cit. on p. 52). 
[82] M. Earnshaw, K. Shelby, H. Lee, Y. Oh, and M. Simon, "Physical Layer Framing for ATSC 3.0", IEEE Trans. Broadcast., vol. 62, no. 1, pp. 263270, 2016 (cit. on pp. 52, 128).

[83] "Frequency and Network Planning Aspects of DVB-T2", European Broadcasting Union, Tech. Rep. 3348, 2013 (cit. on pp. 52, 54, 84).

[84] J. Montalban et al., "Cloud Transmission: System Performance and Application Scenarios", IEEE Trans. Broadcast., vol. 60, no. 2, pp. 170-184, 2014 (cit. on p. 58).

[85] M. K. Ozdemir and H. Arslan, "Channel Estimation for Wireless OFDM Systems", IEEE Communications Surveys $\&$ Tutorials, vol. 9, no. 2, pp. 18-48, 2007 (cit. on p. 59).

[86] S. Coleri, M. Ergen, A. Puri, and A. Bahai, "Channel estimation Techniques Based on Pilot Arrangement in OFDM Systems", IEEE Trans. on Broadcast., vol. 48, no. 3, pp. 223-229, 2002 (cit. on p. 60).

[87] P. Hoeher, S. Kaiser, and P. Robertson, "Two-Dimensional PilotSymbol-Aided Channel Estimation by Wiener Filtering", in 1997 IEEE International Conference on Acoustics, Speech, and Signal Processing, vol. 3, 1997, 1845-1848 vol.3 (cit. on pp. 60, 76, 161, 180).

[88] I. Eizmendi, G. Prieto, G. Berjon-Eriz, I. Landa, and M. Velez, "Empirical DVB-T2 Thresholds for Fixed Reception", IEEE Trans. on Broadcast., vol. 59, no. 2, pp. 306-316, 2013 (cit. on p. 65).

[89] J. J. Gimenez, E. Stare, S. Bergsmark, and D. Gomez-Barquero, "Time frequency slicing for future digital terrestrial broadcasting networks", IEEE Transactions on Broadcasting, vol. 60, no. 2, pp. 227-238, 2014 (cit. on pp. 78,87 ).

[90] C. Regueiro et al., "SHVC and LDM Techniques for HD/UHD TV Indoor Reception", in 2015 IEEE International Symposium on Broadband Multimedia Systems and Broadcasting (BMSB), 2015, pp. 1-6 (cit. on pp. 79,101$)$. 
[91] J. J. Gimenez, E. Stare, S. Bergsmark, and D. Gomez-Barquero, "Advanced Network Planning for Time Frequency Slicing (TFS) Toward Enhanced Efficiency of the Next-Generation Terrestrial Broadcast Networks", IEEE Trans. Broadcast., vol. 61, no. 2, pp. 309-322, 2015 (cit. on p. 79).

[92] "Defining Spectrum Requirements of Broadcasting in the UHF Band", European Broadcasting Union, Tech. Rep. 015, 2012 (cit. on p. 84).

[93] J. Ohm, G. Sullivan, H. Schwarz, T. K. Tan, and T. Wiegand, "Comparison of the Coding Efficiency of Video Coding Standards - Including High Efficiency Video Coding (HEVC)", IEEE Trans. Circuits and Systems for Video Technology, vol. 22, no. 12, pp. 1669-1684, 2012 (cit. on p. 84).

[94] J. J. Gimenez, D. Gozalvez, D. Gomez-Barquero, and N. Cardona, "Statistical Model of Signal Strength Imbalance Between RF Channels in DTT Network", Electronics Letters, vol. 48, no. 12, pp. 731-732, 2012 (cit. on pp. 85, 91).

[95] E Garro, J. J. Gimenez, D. Gomez-Barquero, and S. I. Park, "Performance Evaluation of Layer Division Multiplexing (LDM) Combined with Time Frequency Slicing (TFS)", in 2015 IEEE International Symposium on Broadband Multimedia Systems and Broadcasting (BMSB), Ghent, Belgium, 2015 (cit. on p. 86).

[96] D. Gozalvez, D. Gomez-Barquero, D. Vargas, and N. Cardona, "Combined Time, Frequency and Space Diversity in DVB-NGH", IEEE Trans. Broadcast., vol. 59, no. 4, pp. 674-684, 2013 (cit. on p. 87).

[97] "Propagation Effects Relating to Terrestrial Land Mobile and Broadcasting Services in the VHF and UHF Bands", ITU, Recommendation ITU-R P.1406-1, 2007 (cit. on p. 91).

[98] A. Mattsson, "Single Frequency Networks in DTV", IEEE Trans. Broadcast., vol. 51, no. 4, pp. 413-422, 2005 (cit. on p. 100).

[99] D. Plets et al., "On the Methodology for Calculating SFN Gain in Digital Broadcast Systems", IEEE Trans. Broadcast., vol. 56, no. 3, pp. 331339, 2010 (cit. on p. 100). 
[100] G. Santella, R. D. Martino, and M. Ricchiuti, "Single Frequency Network (SFN) Planning for Digital Terrestrial Television and Radio Broadcast Services: the Italian Frequency Plan for T-DAB", in 2004 IEEE 59th Vehicular Technology Conference (VTC), vol. 4, 2004, pp. 23072311 (cit. on p. 101).

[101] J. Robert and J. Zollner, "Multiple-Input Single-Output Antenna Schemes for DVB-NGH", in Next Generation Mobile Broadcasting, D Gomez-Barquero, Ed., Boca Raton, FL, USA: CRC Press, 2013, pp. 581-608 (cit. on pp. 101, 102, 126).

[102] E. Garro, J. J. Gimenez, S. I. Park, and D. Gomez-Barquero, "Layered Division Multiplexing With Multi-Radio-Frequency Channel Technologies", IEEE Trans. Broadcast., vol. 62, no. 2, pp. 365-374, 2016 (cit. on p. 101).

[103] D. Vargas, D. Gozalvez, D. Gomez-Barquero, and N. Cardona, "MIMO for DVB-NGH, the next generation mobile TV broadcasting", IEEE Communications Magazine, vol. 51, no. 7, pp. 130-137, 2013 (cit. on pp. 104, 126).

[104] T. Shitomi, E. Garro, K. Murayama, and D. Gomez-Barquero, "MIMO Scattered Pilot Performance and Optimization for ATSC 3.0", IEEE Trans. Broadcast., vol. in press, 2018 (cit. on pp. 108, 128, 159).

[105] L. Zhang et al., "Capacity Analysis of LDM-based DTV System with Flexible MIMO Configuration", in 2016 IEEE International Symposium on Broadband Multimedia Systems and Broadcasting (BMSB), 2016, pp. 1-7 (cit. on p. 124).

[106] E. Telatar, "Capacity of Multi-antenna Gaussian Channels", European Trans. on Telecomm., vol. 10, no. 6, (cit. on pp. 124, 166).

[107] L. Kahn, "Correspondence", Proceedings of the IRE, vol. 42, no. 11, pp. 1698-1704, 1954 (cit. on p. 126).

[108] P. Moss and T. Y. Poon, "Overview of the Multiple-Input MultipleOutput Terrestrial Profile of DVB-NGH", in Next Generation Mobile Broadcasting, D Gomez-Barquero, Ed., Boca Raton, FL, USA: CRC Press, 2013, pp. 549-580 (cit. on pp. 127, 184). 
[109] J. Jang, D. H. Kim, and Y. H. Lee, "Open-Loop Precoder for Spatial Multiplexing in MIMO Channels with Phase Correlation", Electronics Letters, vol. 50, no. 23, pp. 1766-1768, 2014 (cit. on p. 128).

[110] E. Garro et al., "Layered Division Multiplexing with Distributed Multiple-Input Single-Output Schemes", IEEE Trans. Broadcast., vol. in press, 2018 (cit. on pp. 128, 141, 162).

[111] P. Fertl, J. Jalden, and G. Matz, "Performance Assessment of MIMOBICM Demodulators Based on Mutual Information", IEEE Trans. on Signal Processing, vol. 60, no. 3, pp. 1366-1382, 2012 (cit. on p. 137).

[112] B. M. Lim et al., "Performance Evaluation of Frequency Interleaver in ATSC 3.0", in 2016 IEEE International Symposium on Broadband Multimedia Systems and Broadcasting (BMSB), 2016, pp. 1-2 (cit. on p. 138).

[113] P. Moss, T. Y. Poon, and J. Boyer, A Simple Model of the UHF CrossPolar Terrestrial Channel for DVB-NGH, White Paper, British Broadcasting Corporation, 2011 (cit. on pp. 140, 167).

[114] P. Moss, 2-by-2 MIMO Fixed Reception Channel Model for Dual-Polar Terrestrial Transmission, White Paper, British Broadcasting Corporation, 2008 (cit. on pp. 141, 167).

[115] L. Zheng and D. N. C. Tse, "Diversity and Multiplexing: A Fundamental Tradeoff in Multiple-Antenna Channels", IEEE Trans. on Information Theory, vol. 49, no. 5, pp. 1073-1096, 2003 (cit. on p. 145).

[116] J. J. Gimenez, D. Gomez-Barquero, J. Morgade, and E. Stare, "Wideband Broadcasting: A Power-Efficient Approach to 5G Broadcasting", IEEE Communications Magazine, vol. 56, no. 3, pp. 119-125, 2018 (cit. on pp. 183, 185).

[117] ATSC Recommended Practice: Guidelines for the Physical Layer Protocol, ATSC, 2018 (cit. on p. 183).

[118] J. Montalban et al., "Asynchronous N-Layered Division Multiplexing (N-LDM)", in 2016 IEEE International Symposium on Broadband Mul- 
timedia Systems and Broadcasting (BMSB), Nara, Japan, 2016 (cit. on p. 184).

[119] T. Fuse, H. Otsubo, A. Nakamura, and M. Itami, in 2017 IEEE International Symposium on Broadband Multimedia Systems and Broadcasting (BMSB), title=A Study on LDM Partial Reception for Next Generation Band Segmented Digital Terrestrial TV Broadcasting, Cagliari, Italy, 2017 (cit. on p. 185).

[120] H. Yamamoto, A. Nakamura, and M. Itami, in 2018 IEEE International Symposium on Broadband Multimedia Systems and Broadcasting (BMSB), title=A Study on LDM-BST-OFDM using Punctured LDPC Code in Partial Reception Band, Valencia, Spain, 2018 (cit. on p. 185).

[121] D. Lecompte and F. Gabin, "evolved Multimedia Broadcast/Multicast Service (eMBMS) in LTE-Advanced: Overview and Rel-11 Enhancements", IEEE Communications Magazine, vol. 50, no. 11, pp. 68-74, 2012 (cit. on p. 186).

[122] W. Zhang et al., "Convergence of a Terrestrial Broadcast Network and a Mobile Broadband Network", IEEE Communications Magazine, vol. 56, no. 3, pp. 74-81, 2018 (cit. on p. 186).

[123] S. M. R. Islam, N. Avazov, O. A. Dobre, and K. S. Kwak, "PowerDomain Non-Orthogonal Multiple Access (NOMA) in 5G Systems: Potentials and Challenges", IEEE Communications Surveys Tutorials, vol. 19, no. 2, pp. 721-742, 2017 (cit. on p. 186).

[124] L. Zhang, Y. Wu, G. K. Walker, W. Li, K. Salehian, and A. Florea, "Improving LTE eMBMS With Extended OFDM Parameters and LayeredDivision-Multiplexing", IEEE Trans. Broadcast., vol. 63, no. 1, pp. 3247, 2017 (cit. on p. 186).

[125] L. Zhang et al., "Layered-Division Multiplexing: An Enabling Technology for Multicast/Broadcast Service Delivery in 5G", IEEE Communications Magazine, vol. 56, no. 3, pp. 82-90, 2018 (cit. on p. 186).

[126] Z. Ding, Y. Liu, J. Choi, Q. Sun, M. Elkashlan, C. L. I, and H. V. Poor, "Application of Non-Orthogonal Multiple Access in LTE and 5G 
Networks", IEEE Communications Magazine, vol. 55, no. 2, pp. 185191, 2017 (cit. on p. 186). 
